\title{
Probabilistic Analysis and Design of Freeway Deceleration Speed Change Lanes
}

\author{
A thesis submitted to \\ the Faculty of Graduate and Postdoctoral Affairs \\ in Partial Fulfillment of the requirements for the degree \\ Master of Applied Science in Civil Engineering
}

by

Ahmed Abdelnaby

Bachelor of Science in Civil Engineering

Department of Civil and Environmental Engineering Carleton University

Ottawa-Carleton Institute of Civil and Environmental Engineering

January 2014

(C)2014 Ahmed Abdelnaby 


\begin{abstract}
In highway design, knowledge about the design parameters and inputs is imperfect. Current geometric design guides provide deterministic methods for the design requirements by using conservative values to consider uncertainty. The design of freeway deceleration speed change lanes (SCLs) depends on the manner of deceleration, initial speed, and final speed at the SCL. SCL length should provide drivers with enough distance to diverge at a reasonable speed and decelerate comfortably. The purpose of this research is to develop probabilistic methodology for evaluating and designing freeway deceleration SCLs using reliability analysis. Models were developed to evaluate the operational performance of SCLs using field data. Three different methodologies were used for evaluating SCL length. PNC, which corresponds to the probability that drivers require a deceleration length longer than what is provided at the SCL, was calculated for each study site. Design graphs were developed to design based on PNC for lengths below $300 \mathrm{~m}$.
\end{abstract}




\section{Acknowledgements}

I would like to truthfully thank God (Allah) for helping me accomplish this research. Without him, this would not be possible. I would like also to convey my sincere gratitude and appreciation to my thesis supervisor, Professor Yasser Hassan for his patience and encouragement during my study period. The endless support and guidance of Professor Yasser Hassan helped me to reach to the highest levels of confidence in my work. I would like to dedicate this thesis to my dearest father (Mr. Salah Khalil), beloved mother (Mrs. Manal AbdulAziz), and my other family members for their moral and financial support for the past two years. Their encouragement was a huge motivation to complete this thesis. Also, the financial support of the National Sciences and Engineering Research Council of Canada (NSERC) is acknowledged. 


\section{Table of Contents}

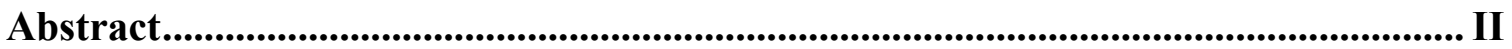

Achnowledgements .....................................................................................................II

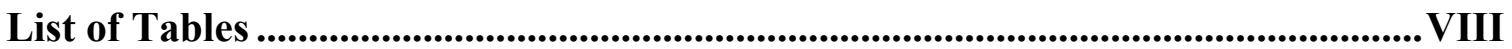

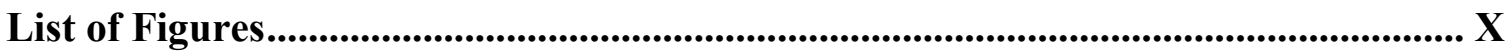

Chapter One: Introduction...........................................................1

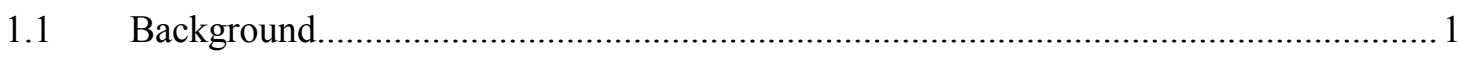

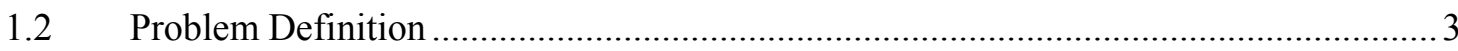

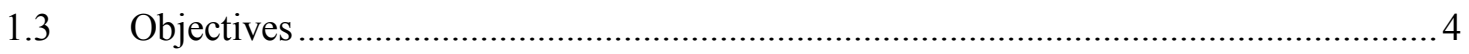

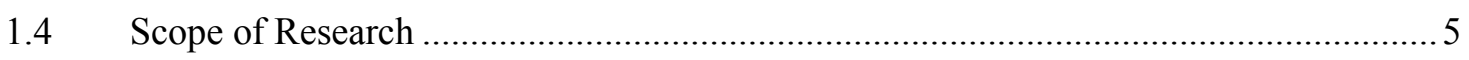

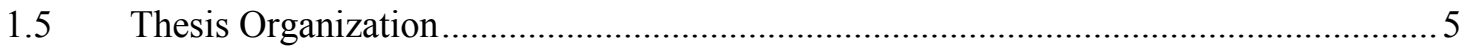

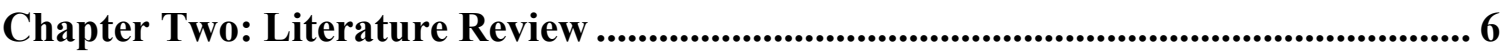

2.1 Interchanges and Speed Change Lanes in Design Guides......................6

2.2 Driver Behaviour..................................................... 15

2.3 Safety and Operational Performance of SCLs.............................21

2.4 Reliability Analysis in Highway Design...................................26

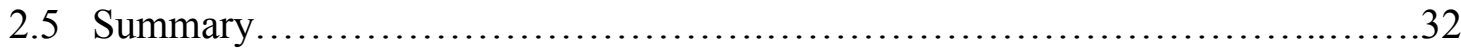

Chapter Three: Data Description ................................................................................. 34

3.1 Study Area and Selected Sites....................................... 34

3.2 Geometric Characteristics of the Study Sites............................. 37

3.3 Available Speed Data............................................... 37

3.4 Data Reprocessing .................................................... 42 
3.5 Collision Data.

Chapter Four: Data Analysis....................................................54

4.1 Summary of Reprocessed Data......................................54

4.2 Statistical Tests on Reprocessed Data.....................................56

4.2.1 Distribution Testing of SCL Speed Profiles............................56

4.2.2 ANOVA Single Factor Mean Tests..............................58

4.3 Comparison of Original and Reprocessed Data............................61

4.3.1 Time and Distance to Decelerate................................62

4.3.2 Final Speeds...............................................63

4.3.3 Initial and Gore Speeds.....................................65

4.3.4 Deceleration Distance and Total Travelled Distance ...................66

4.3.5 Overall Deceleration Rate......................................67

4.3.6 Correlation Between Variables...................................69

Chapter Five: Probabilistic Methodology ............................................................... 71

5.1 Reliability Analysis................................................ 72

5.2 Limit State Function................................................. 74

5.3 First Order Second Moment (FOSM) ................................. 77

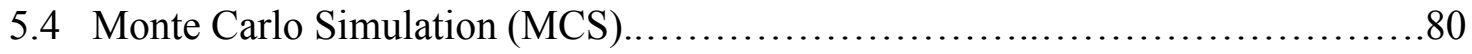

5.5 First Order Reliability Analysis (FORM) $\ldots \ldots \ldots \ldots \ldots \ldots \ldots \ldots \ldots \ldots \ldots \ldots \ldots . \ldots \ldots$

Chapter Six: Results and Discussion .............................................................. 92

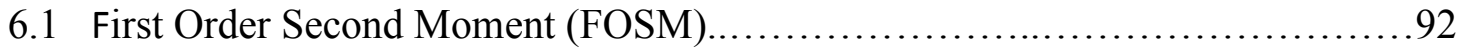

6.2 Monte Carlo Simulation (MCS) ........................................ 94 
6.3 First Order Reliability Analysis (FORM) ............................... 97

6.4 Comparison Between FOSM, MCS, and FORM.............................98

6.4 Relationship to Safety........................................... 102

Chapter Seven: Design Application ......................................................................... 109

7.1 Initial Assumptions and Regression Models................................109

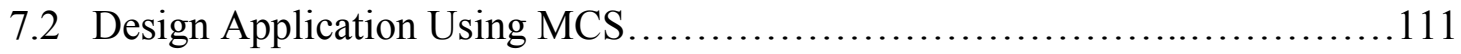

Chapter Eight: Conclusions and Recommendations ............................................. 115

8.1 Conclusions............................................................ 115

8.2 Recommendations for Future Studies.................................... 117

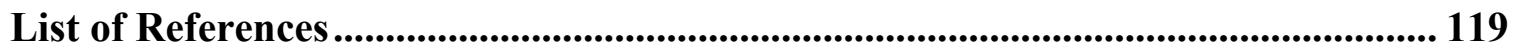

Appendices................................................................................................................................. 126

Appendix 1: Summary of Geometric Data of Study Sites ..................................... 127

Appendix 2: Script Estimating Deceleration Point ............................................... .129

Appendix 3: Final Dataset After Reprocessing the Data ..................................... 134

Appendix 4: Initial Speeds ANOVA Test at Study Sites .................................... 165

Appendix 5: FRL and Diverge Speeds ANOVA Test at Each Site......................... 169

Appendix 6: Multiple Linear Regression of Deceleration Rate Used in MCS.......... 174

Appendix 7: Diagnostics of Decelration Rate Regression Used in MCS ................. 183

Appendix 8: Multiple Linear Regression between Mean of the Parameters and

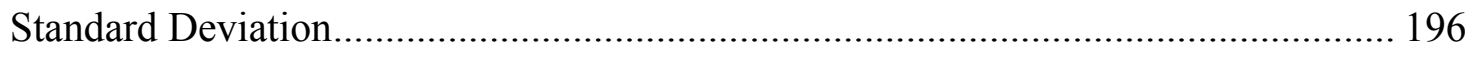

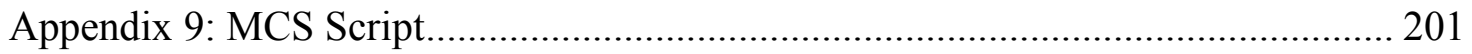

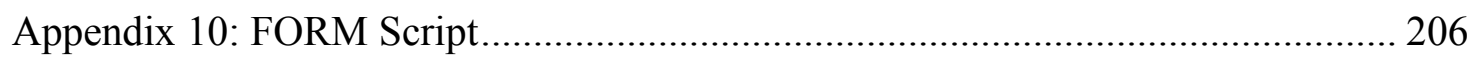




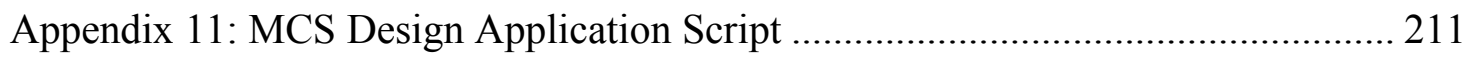

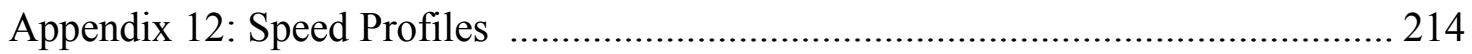

Appendix 13: Frequency of Demand Length Output for MCS Using the Dataset of

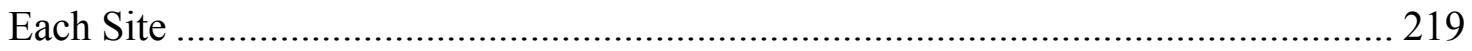




\section{List of Tables}

Table 2.1 : Minimum Deceleration Lengths for Flat Grades with 2\% or Less, Adopted

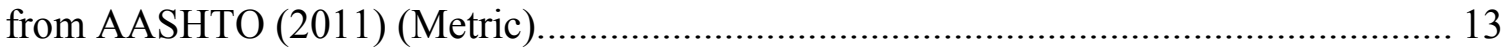

Table 2.2: Design Length for Deceleration, Adopted from TAC (1999). ........................ 13

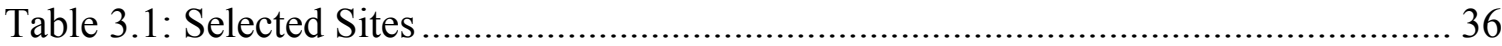

Table 3.2: Available Vehicle Speed Profiles at Every Site. ......................................... 52

Table 3.3: Summary of Collision Data by Site ............................................................ 53

Table 4.1: Distribution of Input Parameters on Study Sites. ........................................ 55

Table 4.2: Distribution Testing Summary of Reprocessed Data .................................. 57

Table 4.3: Summary of Mean FRL, Diverge, and Initial Speeds .................................. 59

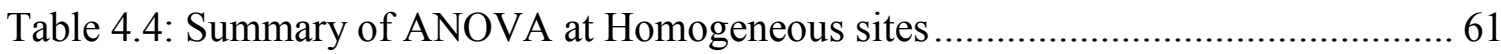

Table 4.5: Mean Final Speed at Each Site and Relative Ramps Advisory Speeds.......... 64

Table 4.6: Summary of Correlation Coefficients and Corresponding P-values before

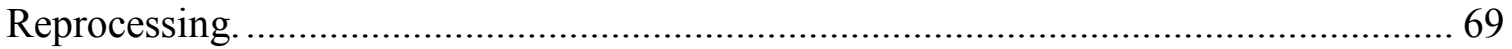

Table 4.7: Summary of correlation Coefficients and Corresponding $P$-values after

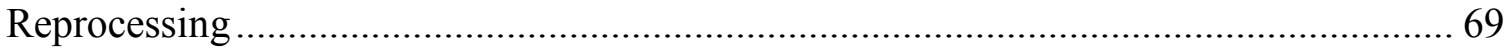

Table 5.1: Linear Regression for the Dataset of All Sites Combined.............................. 81

Table 5.2: Regression Coefficients for Prediction of Individual Vehicle's Deceleration

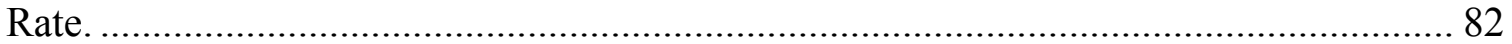

Table 6.1: Results of FOSM Using the Dataset of Each Site. ..................................... 93

Table 6.2: Results of FOSM Using the Dataset of All Sites Combined......................... 94

Table 6.3: MCS Results Using the Dataset of Each Site's........................................ 96

Table 6.4: MCS Results Using the Dataset of All sites Combined. ............................... 96 
Table 6.5: FORM results Using the Dataset of Each Site.

Table 6.6: FORM results Using the Dataset of Each Site All Sites Combined............... 96 


\section{List of Figures}

Figure 2.1: Limited Type SCL, Parallel Configuration.................................................. 10

Figure 2.2: Limited Type SCL, Taper Configuration.................................................. 10

Figure 2.3: Extended Type Deceleration SCL......................................................... 12

Figure 2.4: Example of Type A Weaving (Adopted from HCM, 2000).......................... 16

Figure 2.5: Example of Type B weaving (Adopted from HCM, 2000).......................... 17

Figure 2.6: Example of Type C Weaving (Adopted from HCM, 2000)......................... 17

Figure 2.7: Typical ramp-weave segment based on the HCM definition of weaving

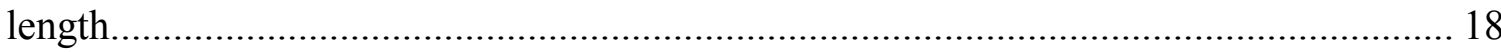

Figure 2.8: Part of Recommended Minimum Ramp Terminal Spacing, (Adopted form

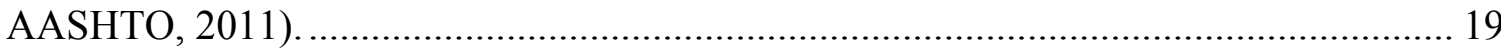

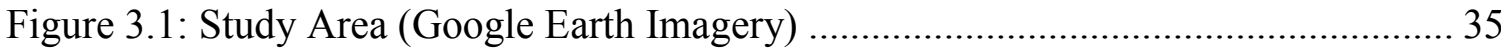

Figure 3.2: Speed Profile Sample for a passenger car on Bronson Ave. W-NS ............... 40

Figure 3.3: Summary of Speed Profiles on Bronson Ave. W-NS ..................................... 41

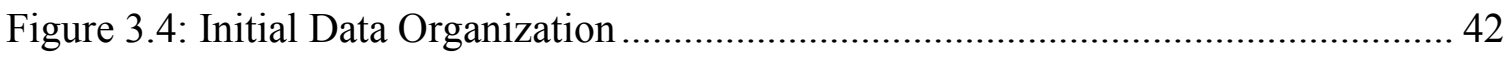

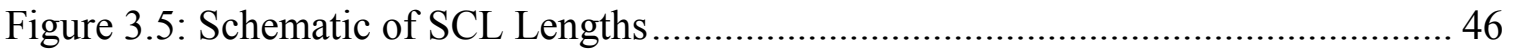

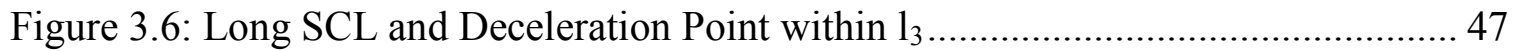

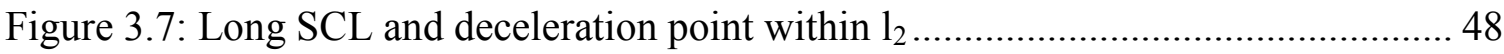

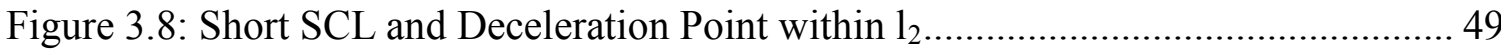

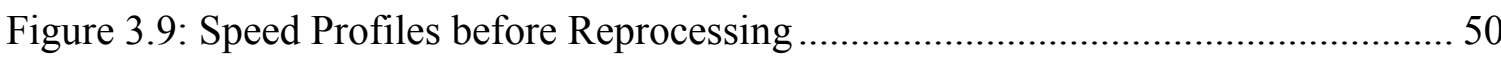

Figure 3.10: Final Speed Profiles after Reprocessing..................................................... 50

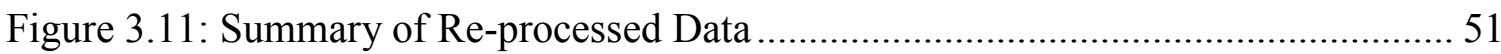


Figure 4.1: Time Spent on the SCL before Decelerating (dots represent each vehicle and cross represents the mean value of vehicles at each site).

Figure 4.2: Distance Spent on the SCL before Decelerating (dots represent each vehicle and cross represents the mean value of vehicles at each site). 63

Figure 4.3: Ramp's Advisory Speeds and Mean Final Speeds at All Sites...................... 64

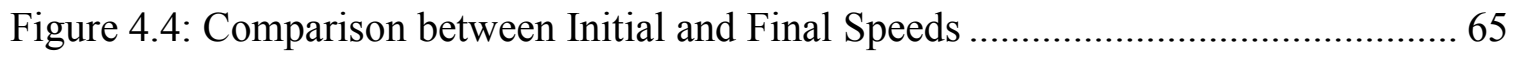

Figure 4.5: Deceleration Distance and TotalTravelled Distance................................. 66

Figure 4.6: Mean Overall Deceleration Rate before and after Reprocessingthe Data...... 68

Figure 4.7: Standard Deviation of Overall Deceleration Rate before and after

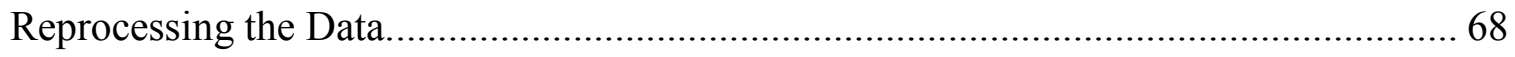

Figure 5.1: Speed Profiles of Individual Vehicles at Bronson Ave. (W-NS) .................. 76

Figure 5.2: Line of Equality between Reprocessed Deceleration Rate and Predicted

Deceleration Rate Using Equation 5.10 for All SCLs Combined. ............................... 83

Figure 5.3: Residuals Plot for Each Predicted Deceleration Rate Using Equation 5.10 at

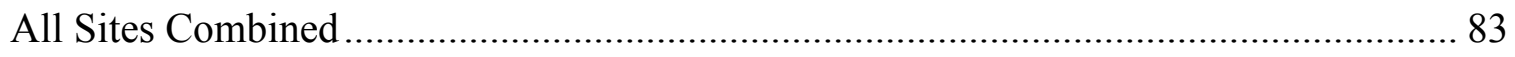

Figure 5.4: Residuals Histogram for All Sites Combined ....................................... 84

Figure 6.1: Mean Demand Length Using FOSM and MCS Using the Dataset of Each

Site.

Figure 6.2: Standard Deviation of Demand L Using FOSM and MCS using the Dataset of each site. 99

Figure 6.3: PNC Using the Dataset of Each Site (FOSM, MCS, and FORM). 100 Figure 6.4: PNC Using the Dataset of All Sites Combined (FOSM, MCS, and FORM). 
Figure 6.5: FOSM PNC Results Using the Dataset of Each Site and Total Collisions

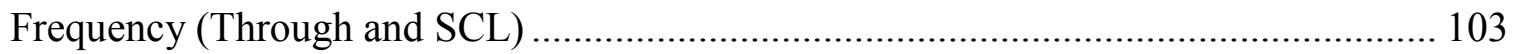

Figure 6.6: FOSM PNC Results Using the Dataset of Each Site and SCL Collisions

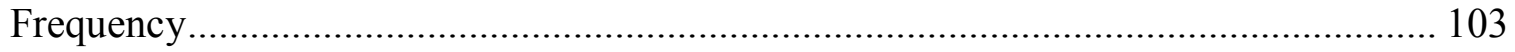

Figure 6.7: MCS PNC Results Using the Dataset of Each Site and Total Collisions

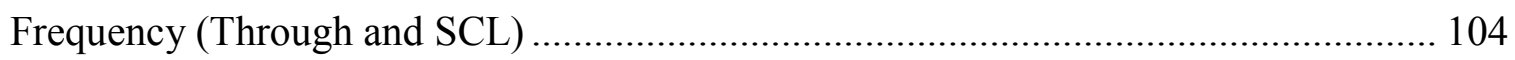

Figure 6.8: MCS PNC Results Using the Dataset of Each Site and SCL Collisions

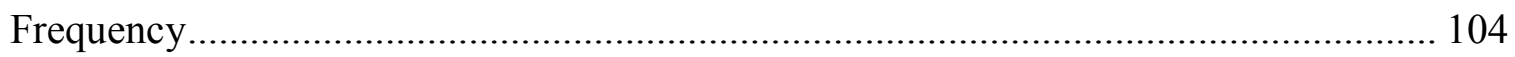

Figure 6.9: FORM PNC Results Using the Dataset of Each Site and Total Collisions

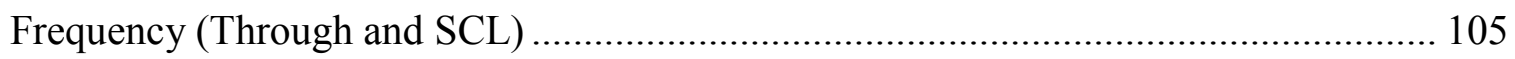

Figure 6.10: FORM PNC Results Using the Dataset of Each Site and SCL Collisions

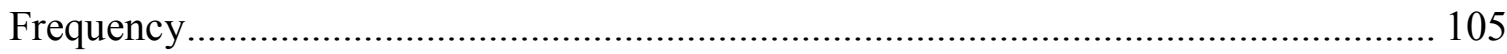

Figure 6.11: FOSM PNC Results Using the Dataset of All Sites Combined and Total

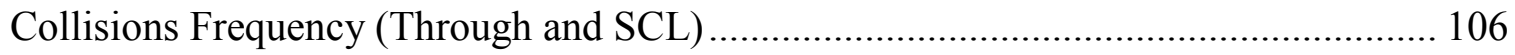

Figure 6.12: FOSM PNC Results Using the Dataset of All Sites Combined and SCL

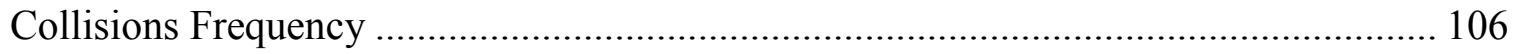

Figure 6.13: MCS PNC Results Using the Dataset of All Sites Combined and Total

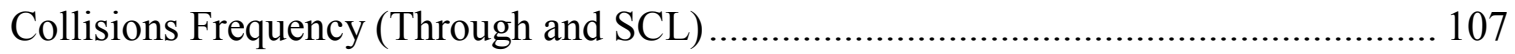

Figure 6.14: MCS PNC Results Using the Dataset of All Sites Combined and SCL

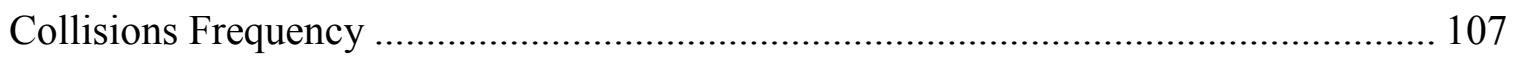

Figure 6.15: FORM PNC Results Using the Dataset of All Sites Combined and Total

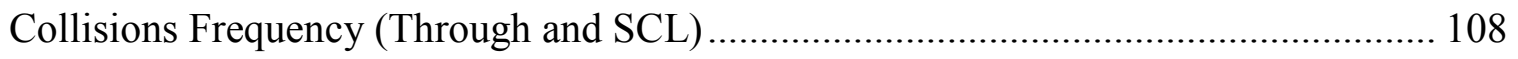


Figure 6. 16: FORM PNC Results Using the Dataset of All Sites Combined and SCL Collisions Frequency

Figure 7.1: Design Curves for Limited SCLs Based on 85th Percentile Final/Gore Speed and PNC for Lengths $\leq 300 \mathrm{~m}$. 113 Figure 7.2: Design Curves for Limited SCLs Based on 85th Percentile Final/Gore Speed and PNC Including $\mathrm{L}>300 \mathrm{~m}$. 114 


\section{Chapter One}

\subsection{Introduction}

\subsection{Background}

Transportation networks are designed to provide safe and efficient movements for people, goods, and services. One of the most important components of transportation networks is roads network. Functionally, roads are classified in American Association of State Highway and Transportation Officials (AASHTO) into principal and minor arterials, collectors and locals (AASHTO, 2011). On the other hand, the Canadian design guide (TAC) classifies a rural network to freeways, arterials, collectors, and locals; while urban networks are classified into freeways, expressways, arterials, collectors, and locals (TAC, 1999). Depending on every road's function, the geometric and traffic characteristics vary. Similarly, the construction and maintenance costs of every class differ based on its importance and role to provide a specific degree of safety and comfort. In accordance, well designed highway elements are anticipated to provide high level of safety and comfort.

According to the Council of Ministers Responsible for Transportation and Highway Safety, the total length of the Canadian national highway system is $38,047.4$ $\mathrm{Km}$, where $91 \%$ is paved, among which $10 \%$ is poorly paved and $22 \%$ is fairly paved (Canada's National Highway System Condition report, 2009). Such a tremendous length indicates a high level of importance for this network of transportation. Safety is an important factor to evaluate potential issues in the network. According to the Road Safety and Motor Vehicle Regulation division in Transport Canada, the number of fatal and 
injury collisions in 2010 is 2,000 and 123,141 respectively, which lead to 2227 fatalities, 11,226 serious injuries, and a total of 170,629 injured victims (Canadian Motor Vehicle Traffic Collision Statistics: 2010, 2012). The statistics also show that the frequency of fatal collisions is higher in rural areas with 1,131 collisions compared to 834 in urban areas, where 35 fatal collisions locations were unknown. On the other hand, frequency of injury collisions is lower in rural areas with 29,716 collisions compared to 91,434 collisions in urban areas, where 1,991 collisions locations were unknown. This difference could be related to the degree of congestion and relative operating speeds in rural and urban areas.

It should be noted that more generous geometric design for highway elements are adapted along arterials compared to collector and local roads as they are designed for higher operational speeds and better mobility. Freeways are an important type of arterial roadways which serve in connecting between different locations with a high degree of mobility. According to the Green Book, "Freeways are arterial highways with full control of access. They are intended to provide for high levels of safety and efficiency in the movement of large volumes of traffic at high speeds (AASHTO, 2011)." Freeways are given full control of access, which means that through traffic has the preference in movement and access connections with other roads are given at selected locations using ramps (AASHTO, 2011). In order to provide a full control of access in a safe and efficient manner, a freeway is connected to another freeway or arterial using an interchange. An interchange consists of loops and ramps at both entrances and exits to get drivers to their destination in an efficient manner. A deceleration lane is provided before an exit terminal to get to the ramp's safe speed and, while an acceleration lane is 
provided after an entrance terminal to help the driver in merging to the roadway safely. Both deceleration and acceleration lanes are auxiliary lanes provided to help the drivers to increase or decrease their speed and are referred to in AASHTO as Speed Change Lanes (SCLs) (AASHTO, 2011). If acceleration SCL has an adequate length, the driver would be able to safely merge onto the freeway at a reasonable speed; otherwise an uncomfortable merging would take place which may result in a conflict or a collision. Similarly, if a deceleration SCL has an adequate length, drivers would be able to reach the ramp's speed safely and comfortably, and otherwise an uncomfortable deceleration maneuver would take place which may lead to a conflict or a collision.

\subsection{Problem Definition}

In transportation engineering, geometric design guides, such as "AASTO (2011)" and "TAC (1999)", use a deterministic approach for the design requirements of road elements, including the design of deceleration SCLs and horizontal and vertical curves. In this approach, conservative percentile values are used to consider uncertainty (e.g. 85th percentile) (Fatema \& Hassan, 2013; Hassan, Sarhan \& Salehi, 2012; Ibrahim \& Sayed, 2011). Using such approach as discussed by Ibrahim \& Sayed (2011) has two main shortcomings.

- First, percentile values selection is not based on safety measures but only on practical experience, which makes the safety margin of the design output to be unknown. 
- Second, there is no quantitative measure of the safety implications resulting from deviating from the design recommendations (e.g., providing sight distance, braking distance ...etc. that is different from design guides).

Furthermore, the reliability of design guides could be debated as they are based on old studies and possibly outdated design values from the 1930s (Fitzpatrick, 2012). On the other hand, probabilistic methodologies using the reliability analysis could be an alternative. Given a level of confidence and the probabilistic characteristics of design parameters, operational performance and safety could be quantified. This would provide more accuracy and flexibility in design (Hassan, Sarhan \& Salehi, 2012; Fatema \& Hassan, 2013).

\subsection{Objectives}

The main purpose of this study is to develop a probabilistic approach for evaluating the operational performance of deceleration SCLs. Probabilistic models are developed considering the distribution of actual driver behaviour based on field data. The premise of the probabilistic approaches is explored using the available collision data. Furthermore, a design aid for deceleration SCLs is developed based on the required operational performance. The data utilized in this study were previously gathered by ElBasha's (2006) for a study on modeling drivers' behavior, which makes this thesis a continuation of El-Basha's (2006) work. El-Basha's (2006) data were reprocessed for the purpose of this study. The probabilistic models are developed using the First-Order Second Moment (FOSM) method, Monte Carlo simulation (MCS), and First-Order Reliability Method (FORM). 


\subsection{Scope of Research}

The scope of this study is limited to freeway interchanges connecting to lower class roads and excludes freeway-to-freeway connections. The data were collected as mentioned earlier by El-Basha (2006) and this research will be limited to the same data collection conditions, such as dry conditions and of peak periods (favorable traffic conditions). Establishing a relation between safety and operational performance is out of the scope of this study; however the study will explore the premise of a relationship between the developed models and safety. Extended SCLs, which are extended from an entrance ramp to an exit ramp, are affected by both merging and diverging vehicles and the associated weaving manoeuvres and are beyond the scope of this research.

\subsection{Thesis Organization}

Chapter 1.0 is an introduction to this study. Chapter 2.0 reviews design guide criteria for SCL design and presents a review of previous studies and research on the topics of safety and operation of SCLs and reliability analysis. Chapter 3.0 describes the data preparation for the purpose of this study. Then, Chapter 4.0 shows analysis of the database obtained for this study. In Chapter 5.0, probabilistic modeling techniques are used to quantify the operational performance of deceleration SCLs. Chapter 6.0 summarizes the results and compares the operational performance results to collision data at the study sites. Chapter 7.0 provides a design application example of the developed design models. Finally, Chapter 8.0 presents a summary of the study findings with recommendations for future research. 


\section{Chapter Two}

\subsection{Literature Review}

This chapter covers a review of design guides criteria and relevant research concerned with freeway diverge areas. Section 2.1 provides a review of current design guides such as the American Association of State Highway and Transportation Officials (AASHTO, 2011) and the Transportation Association of Canada (TAC, 1999). Section 2.2 covers drivers' behaviour on the freeways' diverge areas, specifically freeway right lanes (FRL) and speed change lanes (SCLs). Section 2.3 discusses previous research conducted in safety and operations on SCLs and FRLs. Finally, Section 2.4 covers studies on reliability analysis and design in highway engineering.

\subsection{Interchanges and Speed Change Lanes in Design Guides}

An interchange is defined by AASHTO "as a system of interconnecting roadways in conjunction with one or more grade separations that provides for the movement of traffic between two or more roadways or highways on different levels (AASHTO, 2011)." There are many types of interchanges varying from simple to complex. A designer usually tries to achieve simplicity and uniformity. Some of the basic interchange configurations are available in AASHTO (2011), such as: trumpet, three-leg directional, diamond, partial and full cloverleaf, and many others (AASHTO, 2011). 
One of the main purposes of an interchange is to ensure uninterrupted traffic flow, but many reasons can warrant an interchange. Following is a list of the current warrants from AASHTO (2011):

- Designation by design: Designing a road as a freeway will warrant having an interchange since uninterrupted traffic flow is needed by design.

- Bottlenecks or spot congestions and road user benefit: If the current facility (e.g. an intersection, a roundabout ...) is not servicing the target capacity, an interchange can be warranted. Also, if the delays at at-grade intersections are user costly (in terms of fuel, time, wear on tires, repairs, etc...), an interchange can be warranted.

- Safety: If a current road facility that has serious safety issues, an interchange can be warranted. An example would be a collision prone intersection with many pedestrian incidents.

- Site Topography: If it is more economical to build an interchange than an at-grade intersection due to the topography of the site, an interchange can be warranted. To illustrate, if the cut and fill would cost more than building an interchange due to the type of soil, an interchange could be a feasible solution.

- Traffic Volume: Volumes that exceed the capacity of at-grade intersections would be a warrant. Interchanges are desirable where heavy traffic volumes need to be continuous without interruptions, which would reduce the traffic's exposure rate to conflicts. 
There are other cases where an interchange is required, which are used inside urban areas, such as:

- To provide access to areas that cannot be not served by at-grade means of access within the right of way limits.

- To eliminate a railroad-highway at-grade crossing.

- To provide access to attractions within the proximity of a major arterial, or freeway (For example, a major shopping center on the side of an arterial or freeway).

Interchanges are mainly used to supply access for specific locations, such as the central business district (CBD), diverge into another freeway, etc.... To insure uninterrupted flow on freeways, design guides placed minimum spacing requirement for interchanges allocation. TAC (1999) identifies spacing for interchanges in a highway as 3-8 Km for rural areas and 2-3 Km for urban areas, which is far greater than AASHTO's (2011) values of $3.2 \mathrm{Km}(2 \mathrm{mi})$ in rural and $1.6(1 \mathrm{mi})$ in urban areas. Different countries have their different requirements (Pilko, Bared, Edara \& Kim, 2012); in the United Kingdom (UK), the minimum spacing is a function of speed that supplies the spacing in meters:

Spacing $=3.75 * V$

(Equation 2.1)

Where,

- $\quad V$ is speed in $\mathrm{km} / \mathrm{h}$; and

- Spacing is in meters. 
According to Pilko, Bared, Edara \& Kim (2012), in Germany, the minimum spacing is 2.7 Km, while the Australian recommendations are 1.5-2 $\mathrm{Km}$ in urban areas and 3.1-8.1 $\mathrm{Km}$ in rural areas.

Interchanges consist of three main components: loops, ramps, and speed change lanes (SCL). Generally, a ramp is "turning roadway that connects two or more legs at an interchange" (AASHTO, 2011). A loop is the curved segment that connects two different levels in elevation. A ramp terminal is the parallel portion to the travelled way and it includes:

- Acceleration lane.

- Deceleration lane.

- Weaving section (e.g. in, case of an extended SCL, the weaving area is the length joining the exit and entrance of two ramps in an interchange).

A speed change lane (auxiliary lane) is a separate lane designed for accelerating or decelerating vehicles entering or leaving the road, usually a freeway (AASHTO, 2011). They are warranted on high speed or volume highways or intersections. Deceleration SCLs (scope of this research) can be categorized into two classes; limitedlength and extended-length. A Limited deceleration SCL starts from the taper point as a new lane that diverges from the highway and is categorized into taper and parallel as shown in the figures below. Limited deceleration length in AASHTO (2011) is assumed to extend form the point where the width $\left(\mathrm{W}_{\mathrm{L}}\right)$ of the $\mathrm{SCL}$ is $3.6 \mathrm{~m}$ (distance between the right edge of the through lane to right edge of tapered wedge) to the point of initial curvature of the exit ramp or where the alignment of the ramp roadway departs from the alignment of the freeway. However, for the purpose of this research, limited deceleration 
SCL length included the taper and was measured from the beginning of the taper to the point where pavement edges of the freeway right lane and off-ramp are separated by 1.25 m. Figures 2.1 and 2.2 show the configuration of taper and parallel limited-length SCLs, respectively.

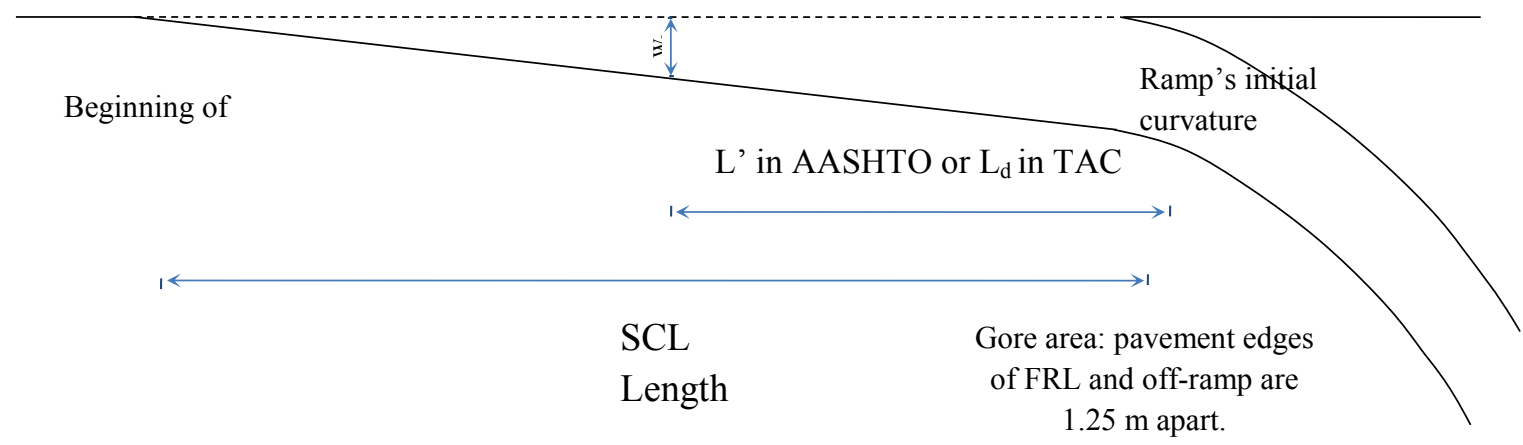

Figure 2.1: Limited Type SCL, Taper Configuration.

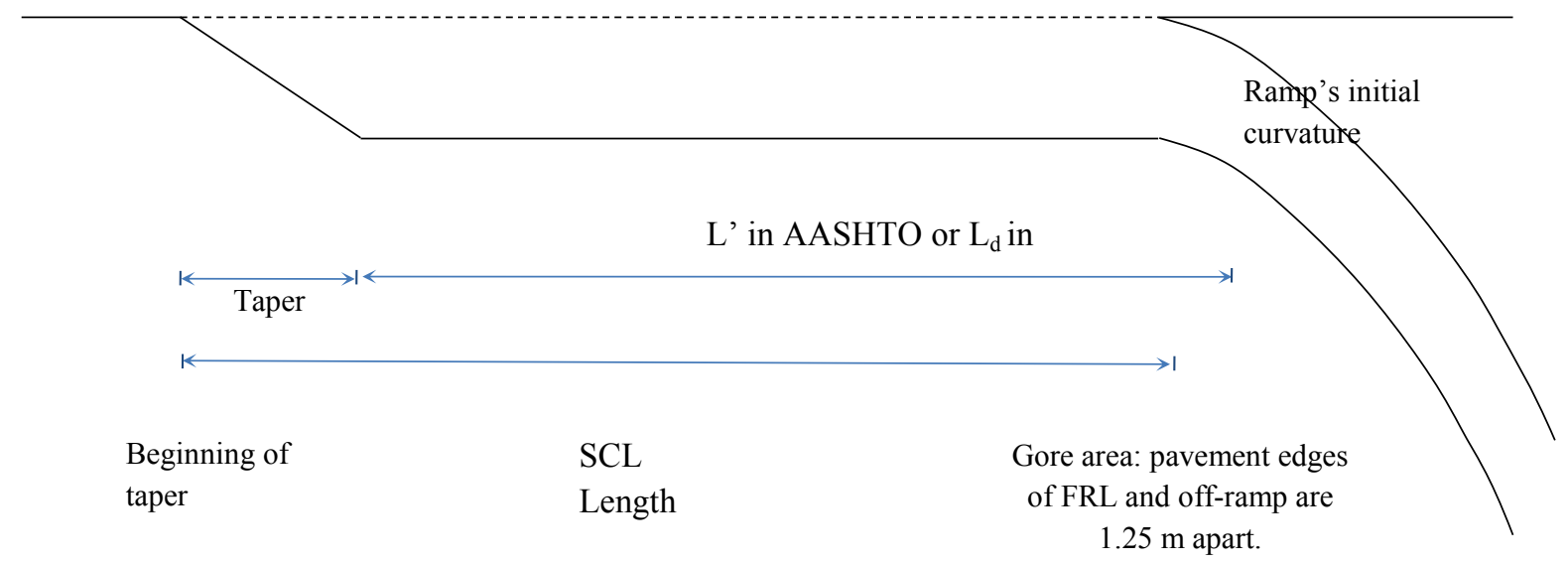

Figure 2.2: Limited Type SCL, Parallel Configuration. 
There are mainly two types of SCLs, parallel and taper. A parallel design as shown in figure 2.2 consists of: $\mathrm{L}_{\mathrm{d}}$ as stated in (TAC, 1999) or L' as stated in (AASHTO, 2011), which is the distance provided for the speed change maneuver between the freeway speed and the ramp. And $\mathrm{L}_{t}$ as called in TAC 1999, which is a gradual change of width and is a transition between the freeway right lane and the SCL. A parallel design is essential where traffic volume on the major highway is near capacity as it provides less confusion to the highway traffic or where through and diverging traffic volumes are sufficiently high (AASHTO, 2011). A tapered deceleration SCL (Figure 2.1) is provided for drivers to slightly decelerate using a sufficient $\mathrm{L}_{\mathrm{d}}$ or L'. According to AASHTO (2011), taper divergence should be between $2-5$ degrees; where in the past edition AASHTO (2004), SCLs should have a taper rate between 8:1 and 15:1 (Longitudinal: Transverse), which is equivalent to $3.8-7.1$ degrees. A taper is designed to allow vehicles to depart the through travel lane using minimum braking. Using a taper that is too short may require vehicles a forced or high deceleration rate (Federal Highway Adminstration, 2004). It should be noted that the term "deceleration rate" is used in the highway literature to refer to the decrease in speed over time or distance; this term is used in the same context throughout this study. It was reported in a study of ramp entrances and exits design that was based on a nationwide NCHRP survey that most states in the United States of America (U.S.) prefer using a taper design for exit ramps. Seventy five percent of the states use a parallel design for entrance ramps and most states comply with AASHTO's minimum length for deceleration SCLs design. However, some states use lengths are less than the minimum recommended by AASHTO (Koepke, 1993). 
An extended SCL, the other class of SCLs, is the lane that connects an entrance ramp with an exit ramp. Extended SCLs are referred to as weaving segments, which according to TAC (1999) should be continued for a distance of $700 \mathrm{~m}$ to $900 \mathrm{~m}$. TAC (1999) does not go beyond HCM (2000) definition of a weaving segment length, which is measured from the point where the right edge of the freeway through lane is $0.6 \mathrm{~m}$ away from the left edge of the entrance ramp, to a point where the freeway right edge and the exit ramp left edge are 3.7 meters apart. On the other hand, AASHTO (2011) depends on HCM (2010) definition of weaving length. In HCM (2010) weaving length is the distance between the end points of any barrier markings that prohibit or discourages lane changing maneuver. For the purpose of this research, extended SCL length is measured from the point where the freeway mainline and the on-ramp pavement edges are separated by 1.25 meters to the same point on the off ramp. Figure 2.3 shows an illustration of an extended SCL based on both HCM 2000 and HCM 2010 definitions.

The Green Book provides minimum requirements to the design of deceleration lengths for exit terminals. Table 2.1 shows part of the table available in the Green Book 2011 edition. Table 2.2 shows part of the minimum length for deceleration SCLs presented in TAC (1999).

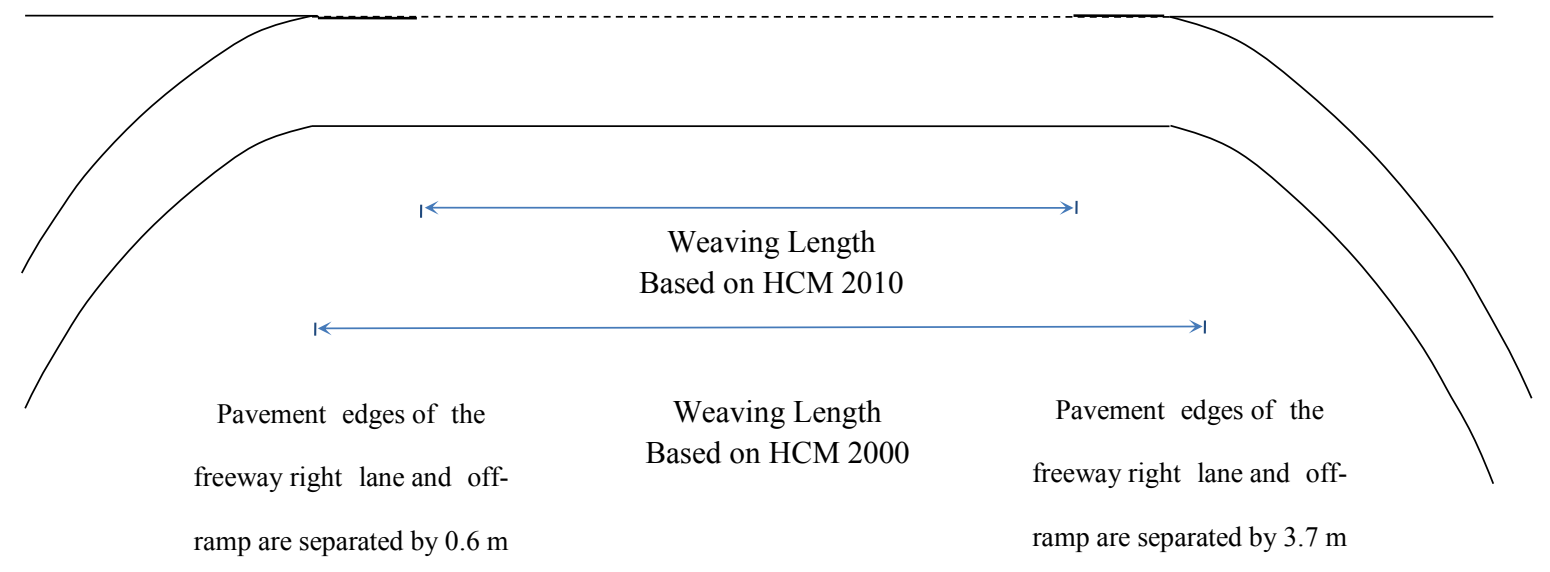

Figure 2.3: Extended Type Deceleration SCL. 
Table 2.1: Minimum Deceleration Lengths for Flat Grades with $2 \%$ or Less, Adopted from AASHTO (2011) (Metric).

\begin{tabular}{|c|c|c|c|c|c|c|c|c|c|}
\hline \multicolumn{10}{|c|}{ Deceleration Length, L (m) for Design Speed of Exit Curve, V' $(\mathrm{Km} / \mathrm{h})$} \\
\hline \multirow{2}{*}{$\begin{array}{c}\text { Highway } \\
\text { Design }\end{array}$} & \multirow{3}{*}{$\begin{array}{c}\text { Speed } \\
\text { Reached, } \\
\mathrm{V}_{\mathrm{a}} \\
(\mathrm{Km} / \mathrm{h})\end{array}$} & $\begin{array}{c}\text { Stop } \\
\text { Condition }\end{array}$ & 20 & 30 & 40 & 50 & 60 & 70 & 80 \\
\hline & & \multicolumn{8}{|c|}{ For Average Running Speed on Exit Curve $\mathrm{V}_{\mathrm{a}}(\mathrm{Km} / \mathrm{h})$} \\
\hline $\begin{array}{c}\text { Speed, V } \\
(\mathrm{Km} / \mathrm{h})\end{array}$ & & 0 & 20 & 28 & 35 & 42 & 51 & 63 & 70 \\
\hline 50 & 47 & 75 & 70 & 60 & 45 & --- & --- & --- & --- \\
\hline 60 & 55 & 95 & 90 & 80 & 65 & 55 & --- & --- & --- \\
\hline 70 & 63 & 110 & 105 & 95 & 85 & 70 & 55 & --- & --- \\
\hline
\end{tabular}

Where; $V=$ design speed of the highway $(\mathrm{Km} / \mathrm{h}), \mathrm{V}_{\mathrm{a}}=$ average running speed of the highway $(\mathrm{Km} / \mathrm{h}), \mathrm{V}^{\prime}=$ design speed of exit curve $(\mathrm{Km} / \mathrm{h}), \mathrm{V}_{\mathrm{a}}{ }_{\mathrm{a}}=$ average $\mathrm{running}$ speed on exit curve $(\mathrm{Km} / \mathrm{h})$.

Table 2.2: Design Length for Deceleration, Adopted from TAC (1999).

\begin{tabular}{|c|c|c|c|c|c|c|c|c|c|c|}
\hline \multicolumn{2}{|c|}{$\begin{array}{l}\text { Speed of Roadway } \\
\qquad(\mathrm{km} / \mathrm{h})\end{array}$} & \multirow{3}{*}{$\begin{array}{l}\text { Length } \\
\text { of Taper } \\
\text { (m) }\end{array}$} & \multicolumn{8}{|c|}{$\begin{array}{l}\text { Length of Deceleration Lane Excluding Taper (m) } \\
\qquad L_{d}\end{array}$} \\
\hline \multirow[t]{2}{*}{ Design } & \multirow{2}{*}{$\begin{array}{l}\text { Assumed } \\
\text { Operating }\end{array}$} & & \multirow{2}{*}{$\begin{array}{l}\text { Stop } \\
\text { Condition }\end{array}$} & \multicolumn{7}{|c|}{$\begin{array}{l}\text { Design Speed of Turning Roadway Curve } \\
\qquad(\mathrm{km} / \mathrm{h})\end{array}$} \\
\hline & & & & 20 & 30 & 40 & 50 & 60 & 70 & 80 \\
\hline 60 & $55-60$ & 55 & $90-115$ & $\begin{array}{l}80- \\
110\end{array}$ & $\begin{array}{l}80- \\
105\end{array}$ & $\begin{array}{l}70- \\
90\end{array}$ & $\begin{array}{l}55- \\
60\end{array}$ & --- & --- & --- \\
\hline 70 & $63-70$ & 65 & $110-145$ & $\begin{array}{c}105- \\
140\end{array}$ & $\begin{array}{c}100- \\
130\end{array}$ & $\begin{array}{l}90- \\
120\end{array}$ & $\begin{array}{l}75- \\
105\end{array}$ & $\begin{array}{l}60- \\
70\end{array}$ & --- & --- \\
\hline 80 & $70-80$ & 70 & $130-170$ & $\begin{array}{l}120- \\
165\end{array}$ & $\begin{array}{l}115- \\
160\end{array}$ & $\begin{array}{c}105- \\
150\end{array}$ & $\begin{array}{l}95- \\
130\end{array}$ & $\begin{array}{l}80- \\
105\end{array}$ & --- & --- \\
\hline
\end{tabular}

The upper limit values are based on deceleration-distance curves in the geometric design standards for Ontario highways 
AASHTO's deceleration SCL lengths were calculated based on old studies conducted on 1930s with the assumption that most drivers are travelling with speeds less than the average running speeds of the highway (Fitzpatrick, Chrysler \& Brewer, 2012); it was argued that these values need to be updated. The 2004 Green Book's deceleration SCL distance values are similar the blue book of 1965 with slight differences (Fitzpatrick, Chrysler \& Brewer, 2012). As the 2011 edition values are close to the 2004's edition in terms of deceleration rates, the similarities of the 2011's edition to the blue book may apply. Fitzpatrick, Chrysler \& Brewer (2012) believe the following equation was used in the deceleration length calculation in the Green Book.

$L=v_{i}-0.5 d_{n}\left(t_{n}^{2}\right)+\frac{v_{i}^{2}-v_{f}^{2}}{2 d}$

Where,

- $L$ is the required length by the SCL users to decelerate,

- $v_{i}$ is initial speed in $\mathrm{m} / \mathrm{s}$,

- $v_{f}$ is driver's final speed after decelerating,

- $d_{n}$ is deceleration rate without brakes in $\mathrm{m} / \mathrm{s}^{2}$,

- $t_{n}$ is deceleration time without brakes, and

- $\quad d$ is deceleration rate with brakes in $\mathrm{m} / \mathrm{s}^{2}$.

Fitzpatrick, Chrysler \& Brewer (2012) believed that Equation 2.2 was used in AASHTO assuming that there is a 3 seconds deceleration without applying brakes that occur on the taper (Fitzpatrick, Chrysler \& Brewer, 2012). In summary, deceleration length calculation in the Green Book assumes a slight deceleration before pressing the brakes. Thus, deceleration rate is not a constant value in AASHTO calculations. 


\subsection{Driver Behaviour}

Drivers' behaviour becomes more complex with the increase of the complexity of the assigned tasks. A limited-length SCL will have less complexity than an extended SCL as weaving manoeuvres may become more challenging on extended SCLs. There is a series of decisions that drivers need to take as they decide to exit a freeway using a limited-length deceleration SCL. First, selecting the diverge point, at which they change lane from the FRL to the SCL, and the corresponding diverge speed. Once on the SCL, a driver may not decelerate immediately if the lane is judged to be too long; slight acceleration or deceleration can take place. Drivers then select a deceleration point to start decelerating, and a corresponding initial deceleration rate. As drivers travel on the SCL, their deceleration rate could increase as they get closer to the gore proximity. Finally, a final speed based on the end conditions of the SCL (e.g., signalized intersection, sharp curve, etc.), would be selected. In case of an extended SCL, in addition to the above tasks, and before entering the SCL, drivers will need to select a satisfactory gap to be able to diverge onto the SCL and get to the ramp, which will depend mainly on the traffic and the configuration of the weaving area.

According to TAC (1999), "weaving sections are roadway segments where the pattern of traffic entering and leaving at contiguous points of access result in vehicle paths crossing each other." Previously, the Highway Capacity Manual HCM (2000) defined three types of weaving sections depending on how the lane change manoeuvre is performed between the SCL and the right lane of the freeway: 
- Type A

○ Almost all vehicles on the ramp are weaving. In this type all weaving vehicles have to make one lane change to complete the manoeuvre.

- Type B

- One through lane occupies weaving vehicles without the need to change lanes. And the other weaving lane requires a maximum of one lane change.

- Type C

One weaving movement does not require a lane change while the other weaving movements need two or more lane changes.

An example of types A, B, and C is shown in Figures 2.4, 2.5, and 2.6, respectively. All Figures associated with each weaving type could be found in HCM (2000).

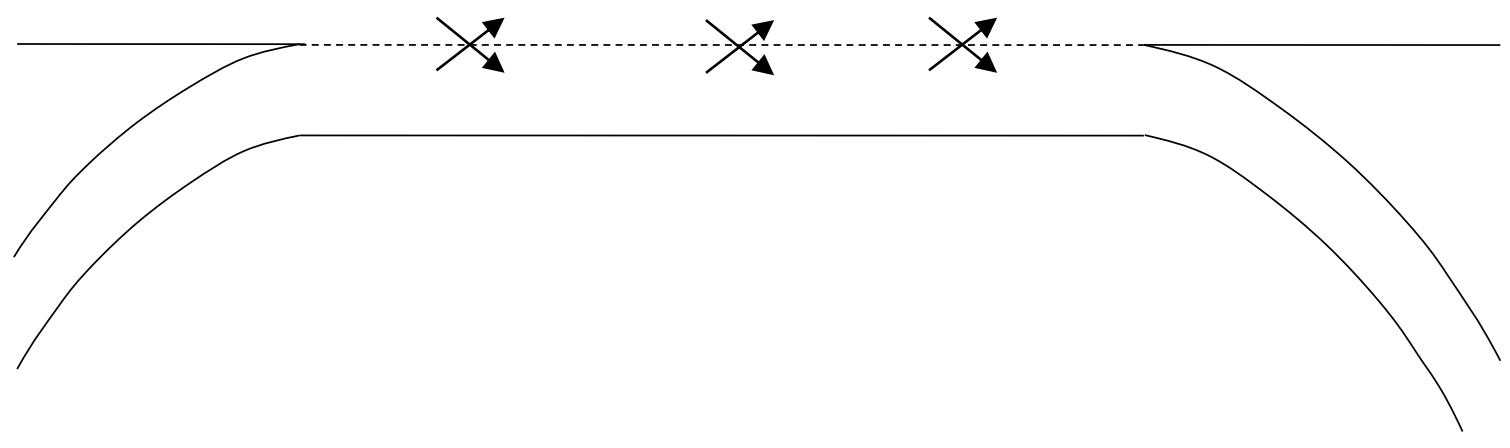

Figure 2.4: Example of Type A Weaving (Adopted from HCM, 2000). 


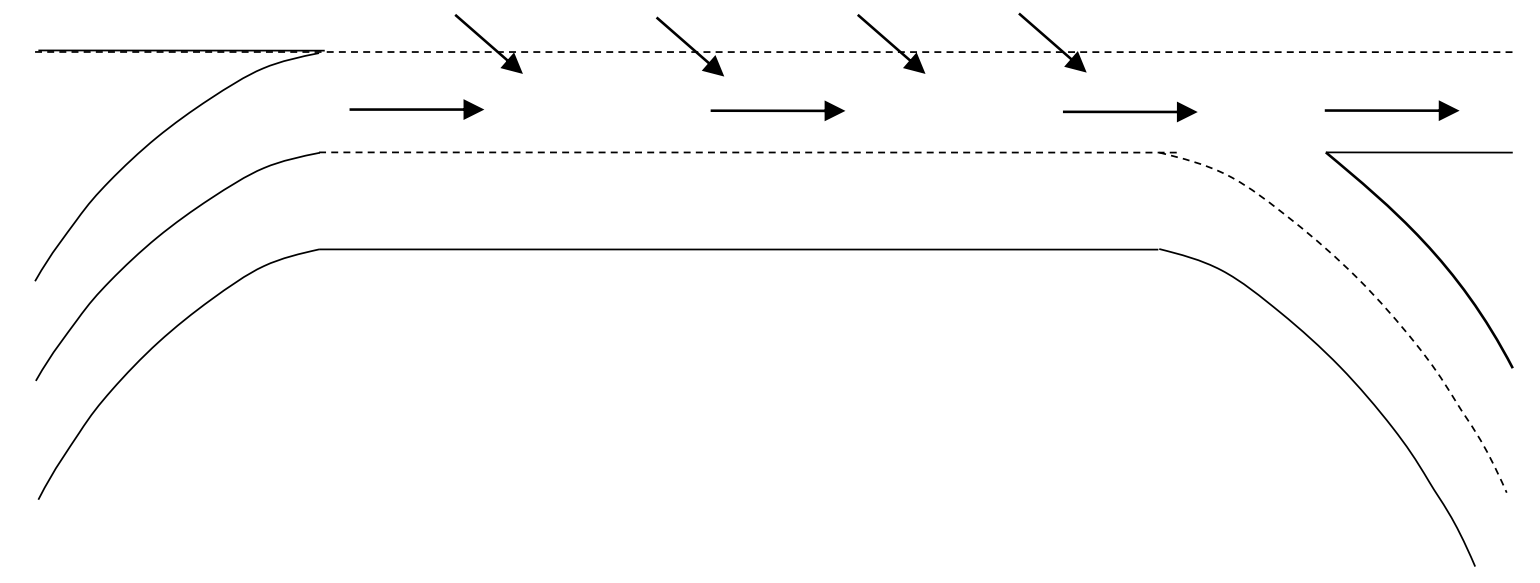

Figure 2.5: Example of Type B weaving (Adopted from HCM, 2000).

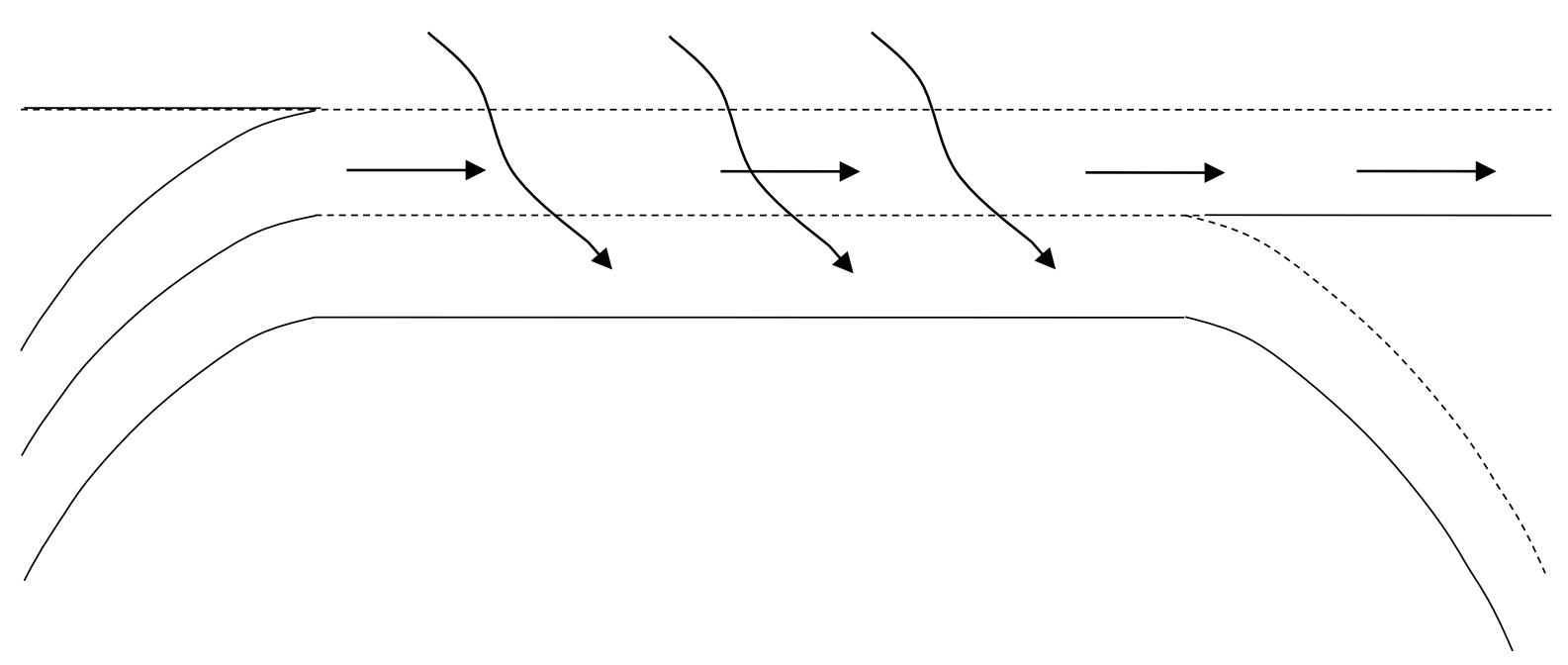

Figure 2.6: Example of Type C Weaving (Adopted from HCM, 2000).

To design a weaving segment, HCM (2000) has a computational process where the Level of Service (LOS) is first defined by the designer. Then an assumed number of lanes and length is taken into the calculation process. After completing the process, the assumptions are verified. In the most recent edition of HCM (2010), weaving is defined as one or more lane change maneuvers, where the number of lanes. A weaving maneuver may be completed with a lane change or without a lane change, which is referred to as 
$N_{W L}$. For a typical ramp weave segment where $\mathrm{N}_{\mathrm{WL}}=2$, there are two lanes where a weaving maneuver may be completed from as shown in Figure 2.7 below.

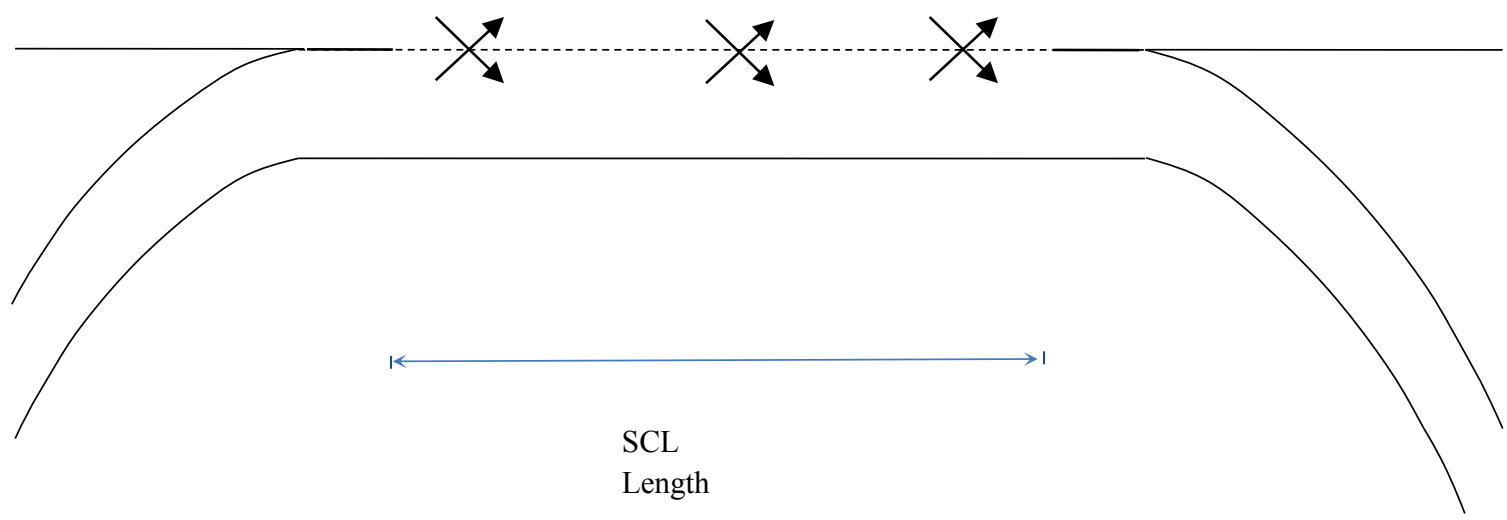

Figure 2.7: Typical Ramp-weave Segment Based on the HCM definition of Weaving Length.

In the new HCM edition, the maximum weaving length is not a fixed length, but depends on the point where the segment's capacity is reached. Maximum length of the weaving segment is a function of the weaving-to-total-volume ratio and the number of weaving lanes as shown in equation 2.3 below.

$L_{M a x}=5,728(1+V R)^{1.6}-1,566 N_{W L}$

(Equation 2.3)

If the segments length is less than the maximum $L_{M a x}$, the segment is treated as a weaving segment; otherwise it would be analyzed as a separate ramp junction and basic segment in between. If the length is less than the maximum, the capacity is then determined and the volume to capacity ratio is then found. If the volume to capacity exceeds one, level of serves (LOS) would be "F"; if the ratio is less than one, the lane changing rates would be determined followed by the average speeds of weaving and non- 
weaving vehicles. Finally LOS is calculated (Bloombertg, 2008; Transportation Research Center, 2010).

Although there is a change in the weaving segments design criteria, TAC (1999) does not go beyond the HCM 2000 calculation methodology to determine the length of weaving segments. On the other hand, AASHTO 2011 refers to HCM 2010 in regards to the length of weaving segments. If the frequency of lane changes is similar to that in an open road, TAC (1999) considers the section as a no weaving segment. The maximum length of weaving areas is $1000 \mathrm{~m}$ (TAC, 1999). Based on TAC, a sufficient weaving length is $800-1000 \mathrm{~m}$ within arterial and freeway interchanges, and $550-700 \mathrm{~m}$ within arterial interchanges.

Weaving is affected by the spacing of the ramps in an interchange. Based on operational experience and the need of flexibility and adequate signing, AASHTO 2011 provides minimum spacing requirements between different successive combinations of ramps, which ranges between $150 \mathrm{~m}(500 \mathrm{ft})$ and $600 \mathrm{~m}(2000 \mathrm{ft})$.

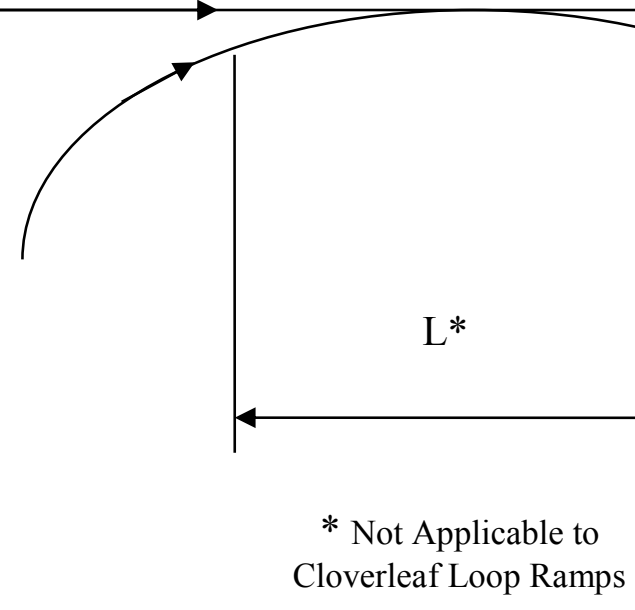

Figure 2.8: Part of Recommended Minimum Ramp Terminal Spacing, (Adopted form AASHTO, 2011). 
Weaving manoeuvres mainly affect extended SCLs operations because of their configuration as shown in the figure 2.6. Also, drivers who decide to use the SCL to overtake a slow vehicle on the FRL then return to the FRL; or drivers who travel back to the FRL form the SCL because they entered the SCL by mistake affect the operations on both extended and long limited-length SCLs. It can be argued that drivers operations on FRL affect operations on the SCL. To illustrate, if for any reason the leading vehicle on a FRL travels with a slow speed, diverge speed onto the SCL of the following vehicle will be slow. Also, available length for deceleration SCLs may affect the operations on the FRL as well. For example, if the SCL length is judged by the driver to be short, deceleration on the FRL could take place. The finding that drivers sometimes decelerate on the FRL when the SCL is short is verified using the field data studied in the NCHRP 730 report (Torbic, et al., 2012).

Deceleration rates on SCLs vary depending on the type of the SCL and its length. AASHTO does not provide a table of assumed deceleration rates (Torbic, et al., 2012). However, NCHRP 730 provided two methodologies to back-calculate these deceleration rates, using both Equation 2.2 and using a constant deceleration approach. It should be noted that the research back-calculated AASHTO's values using recommended deceleration lane lengths for level grades and compared them to deceleration rates measured from the field. Using both approaches, field deceleration rates were lower than the derived AASHTO values (Torbic, et al., 2012). This indicates that AASHTO (2011) assumed higher deceleration rates than what drivers really use.

Drivers' deceleration behaviour was studied in NCHRP 730 report (Torbic, et al., 2012). It was found that drivers do not apply constant deceleration rates while driving on 
the SCL. In another study, speed profile data were collected on deceleration SCLs and FRL and it was found that the magnitude of speed deferential between diverging and through vehicles on FRL depends mainly on the SCL length (El-Basha, Hassan \& Sayed, 2007). This conclusion suggests that SCL length affect whether deceleration starts on the FRL or on the SCL depending mainly on the available SCL length.

\subsection{Safety and Operational Performance of SCLs}

If the driver, for any reason, could not merge with the traffic flow before of the end of acceleration SCL, a conflict may happen. In case the mainline drivers and/or the SCL driver do not perform sufficient accommodating maneuvers a collision may occur. Similarly, if the distance to decelerate was judged to be short, harder deceleration would be applied by the SCL driver; that deceleration might also start on the FRL. In case the following vehicles, which could be on the SCL or the FRL, do not perform accommodating maneuvers, a conflict or a collision could take place. These examples relate operational performance to safety. There are many studies that discussed safety and operational performance on SCLs. Collisions are rare events, thus making them harder to model. Trials to model collision frequency were done in a Federal Highway Administration report; however, it was very hard to model the collision frequency on SCLs as collisions were so low at most locations (Bauer \& Harwood, 1998). Negative binomial regression was followed in modeling collision frequency, which is recently used among safety researchers.

Many studies found that increasing SCLs lengths would increase safety and decreasing lengths would decrease safety. Cirillo (1970) studied the relation of SCL 
lengths to accidents. Data were collected from 20 states for vehicles travelling on interstate highways. The extended SCLs (weaving areas) ranged between $400 \mathrm{ft}$ and 799 $\mathrm{ft}$. Deceleration SCLs were arranged based on their lengths to categories of hundreds (less than $200 \mathrm{ft}$, between $200 \mathrm{ft}$ and $299 \mathrm{ft}, \ldots$, and more than $700 \mathrm{ft}$ ). It was found that longer SCLs and weaving areas have fewer accidents, when the percentage of merging vehicles is more than $6 \%$ of the mainline volume. The results indicated that deceleration SCLs have less reduction in collisions compared to acceleration SCLs, when the SCL length is increased. Even though the number of collisions (collision frequencies) was available in the study, Cirillo (1970) used the accidents rates in the analysis. The analysis carried out for weaving areas considered to some extent the effect of mainline volume on the collision rates by generating collision rate graphs with respect to different ranges of volumes. Also, SCL accident rates graphs were generated with respect to the percent of merging traffic. It should be noted that considering each volume range and percentage of merging traffic separately might have decreased the error of using accident rates; but using rates instead of frequencies could be misleading because locations with lower traffic volumes would have high rates and vice versa. Similar to Cirillo's (1970) findings, a safety evaluation of acceleration and deceleration SCL lengths indicated that deceleration SCLs exhibit higher numbers of accidents compared to acceleration SCLs (Bared, Giering \& Warren, 1999). The authors developed accident prediction models using data from 276 exit and 192 entrance ramps. Their findings could be explained by the complexity of the driver's tasks on deceleration SCLs compared to acceleration SCLs as explained in section 2.2. Similar findings were achieved in Sarhan, Hassan \& Abd El Halim (2008). In addition to the length, traffic exposure was studied as well in order to 
develop safety explicit design graphs (Sarhan, Hassan \& Abd El Halim, 2008). An additional finding of this study is that increasing exposure increases collision frequencies on both acceleration and deceleration SCLs.

Chen, Zhou \& Lin (2012) studied safety on deceleration lanes on 218 sites in the U.S. Simulation was performed taking into account the number of exit lanes, through movement lanes, exiting volume, design speed on the freeways and exists, and the deceleration lane lengths (Chen, Zhou \& Lin, 2012). For both one and two lane exits, crash counts and rates were compared to deceleration lengths. Their study concluded that there is an increasing trend for collisions at longer sites. It should be noted that according to their results collisions decrease with the increase of SCL length; however, when SCLs are very long, collision frequencies and rates start increasing. The authors suggested that from a safety point of view, deceleration SLCs should not be more than $700 \mathrm{ft}$. Many explanations could be argued; for example, in case of long deceleration lanes, drivers may accelerate causing the extra deceleration distance to have no positive safety effect. Also, as mentioned in section 2.2, drivers may utilize the deceleration SCL for weaving maneuvers. On the other hand, from an operational point of view, the authors provided optimal lengths based on the delay reduction for through traffic compared to different lengths of SCLs at different speeds. It was found that these optimal lengths are higher than the Green Book minimum deceleration lengths (Chen, Zhou \& Lin, 2012).

Using a new methodology to determine deceleration lane lengths, Rojas and Garcia (2010) used a conflict indicator named "potential time to lateral or rear-end collision" (PTLRC). This indicator assesses the time between the manoeuvre of the leading vehicle and the collision time if the following vehicle does not perform an 
evasive manoeuvre. They studied 10 sites using 2004-2007 collision data. The relationship between collisions rate and the ratio of actual to design length was studied using multiple linear regression (Rojas \& Garcia, 2010). The conclusion was that in addition to the actual length, collisions depend also on the ratio of actual to design length. On the other hand, conflict severity or intensity on deceleration lanes depends mainly on the ratio of actual to design length only. The finding of this study is that very short and long deceleration lengths are less safe as explained below:

- If design length is $220 \mathrm{~m}$, to reach optimum safety, available length at site should be $220 \mathrm{~m}$.

- If design guide length is more than $220 \mathrm{~m}$, to reach optimum safety, shorter length should be used.

- If design guide length is less than $220 \mathrm{~m}$, to reach optimum safety, longer length should be used.

Even though a new conflict measure that could be used effectively to evaluate operational performance was developed, the reliability of his study could be questioned for the fact that linear regression was used in modeling accidents. It should be noted that using linear regression assumes normal distribution for the collisions, which was proven wrong (Bauer \& Harwood, 1998); using linear regression may not predict the zero collisions due to the value of the intercept; also, using linear regression could result in negative collision frequency, which is not practically possible. Using accident rates as a response variable could be questionable as well; collision frequency should be used instead as explained earlier. Finally, comparing the actual length to the design guide's 
deceleration distance length may not be the best technique to evaluate safety since the safety outcome of the design guides equation is generally unknown.

In a recent study, the probability of forced merging from acceleration SCLs onto the FRL was studied to design for SCLs length considering acceleration and gap acceptance behaviour (Fatema \& Hassan, 2013). In their paper, the authors compared different estimated reliability measures such as mean probability of failed merging percentages of vehicles with PNC equal/greater than zero with the five-year SCL collision frequency at eight study sites. They found that there is a positive trend between reliability measures, such as the mean probability of failed merging associated with the SCL, and collision frequencies on the study sites. Using the developed models in their study, "AASHTO (2004)" and the Canadian design guide "TAC (1999)"minimum lengths were evaluated as well. It was indicated that these minimum lengths may produce a trend of increasing mean PNC as the design speed of the ramp controlling curve increases.

SCL length is not the only factor that affects safety and operational performance. Safety of right turn SCLs for at grade intersections was studied and it was found that geometry, volume, operational characteristics, are main factors that affect safety (Harwood, Bauer, Potts, Torbic \& Richard, 2002). Accident data were collected at 100 intersections with right turn deceleration lanes and 100 intersections without right turn deceleration lanes with similar characteristics. Using Empirical Bayes before and after methodology, it was found that deceleration right-turn lanes improve safety at intersections. In another study, injury severity levels at freeway exit ramps were studied with respect to geometric design, environment, vehicle/driver characteristics, and traffic 
features (Wang, Lu \& Zhang, 2009). After developing different prediction models, it was found that two-lane configurations of exit ramps increase the injury severity while channelization at ramp terminals connecting to cross roads decreases the severity. Safety performance evaluation of left side off-ramps was carried out in a different study (Chena, Zhoub, Zhaob \& Hsuc, 2011):

- Using a conflict study that depends on how maneuvers of freeway and ramp drivers are performed.

- Using crash rates, frequency, and severity of a comparison group of right side off-ramps.

- Using negative binomial collision prediction models (CPMs).

The conflict study indicated that locations with two lanes exclusive off-ramps (two obligatory lanes direct driver to the off-ramps) will have slightly higher conflict occurrence than locations with an optional lane (two lanes direct drivers off ramp, where one of the lanes is shared between the freeway and off ramp). It was found that crash rate, frequency, and severity are higher at left side off-ramps compared to right side off-ramps. Finally, the developed CPMs indicated that as volumes at the freeway and the ramp, or lengths of deceleration lane increase, collision frequency increases. On the other hand, when the ramp's length increases, collision frequency decreases. These findings are only related to left-side off-ramps as a special configuration.

\subsection{Reliability Analysis in Highway Design}

As mentioned earlier, current geometric design guides, such as TAC (1999) and AASHTO (2011), provide deterministic methods for the design requirements based on 
data from the 1930s (Fitzpatrick, Chrysler \& Brewer, 2012). One of the concerns is that these data are outdated. Another concern is that this deterministic approach might lead to an over or under-presentation of the drivers' population, which may raise the previous concerns about the margin of safety and what might happen if the design violates the design guides. On the other hand, reliability analysis techniques provide an alternative probabilistic approach that assesses the performance of the given system based on a sample with known characteristics and confidence level. Operational performance and safety can be quantified by statistical analysis of field data. Using a probabilistic approach can help in providing an assessment of risk and allows for more flexibility in the design. This can be particularly useful in the design and evaluation of SCLs instead of a deterministic approach that traditionally uses a combination of conservative and nonconservative design parameters (Hassan, Sarhan \& Salehi, 2012; Fatema \& Hassan, 2013).

There are many techniques to statistically assess a demand and supply in a system. Most of these techniques are approximations with different accuracies which depend mainly on the formulation of the system and the followed evaluation technique. The use of approximation techniques comes as many engineering systems could not be evaluated using an exact method (the integration technique of the performance function). This is mainly due to the complexity of the limit state function (Section 5.1) and setting their limits (Faber, 2007). Even though, the statistical techniques are approximations, they are normally accurate enough for the engineering applications use. Zhao and Ono (1999) compared different statistical techniques to evaluate the probability of supply exceeding demand. Accuracy of different methods was studied in comparison to a 
simulation technique called Monte Carlo Simulation (MCS) and it was found that with lower number of variables, more linear function's surface, and lower reliability indices, the accuracy of the used methods becomes high. The used methods were First Order Reliability Method (FORM), Second Order Reliability Method (SORM) and they were compared to MCS. These methods are explained later in Chapter 5.0.

Many transportation systems have been studied and researched using reliability analysis. A doctoral thesis gave a design procedure to design for superelevation based on reliability methods (Abia, 2010). Design and evaluation of the reliability of left turn bays lengths at non-signalized intersections was carried out as well using reliability methods. Furthermore, peak hour delays at signalized intersections were studied using reliability analysis in Abia's (2010) thesis as another application of reliability analysis. This study found that designing superelevation rates using reliability methods results in lower rates than what is used in current practice, which can be accounted for as a cost saving with respect to the embankment. Also for left turn bays lengths for non-signalized intersections, the study found that at $95 \%$ level of confidence, the current design practices fail when the degree of saturation, which is the ratio of approaches hourly volume to the capacity of the approach, exceeds $50 \%$. Failure may result in a high level of unsafe braking leading to a conflict or a collision. For the signalized intersection peak hour delays, it was found that using the HCM (2000) methodology, where traffic flow follows a Poisson distribution, for intersection delay analysis is not sufficient. Abia (2010) analyzed signalized intersections that were adjusted to the prevailing traffic conditions. It was found that the HCM (2000) methodology produces more delay (6.2 seconds per vehicle), which results in higher costs. 
Models to examine design features such as, stopping sight distance (SSD), horizontal and vertical curvature, and others were applied to reach a cost effective process for designing roads (Zheng, 1997). Probability of noncompliance of a design criterion was used to test drivers' response to different design alternatives. Zheng (1997) developed a driver, vehicle, roadway, interaction model called the Moving Coordinate System Model (MCSD). The model is a reliability based highway framework that takes into account the vehicle dynamics considerations. The upper limit of that framework is the "racing car model," which is used to represent the maximum theoretical demand of the users (drivers) on the highway system. This upper limit was compared to the road design inputs (supply in the highway system). The racing car model is basically a representation of the theoretical limit of the vehicle at a specific operating condition. Probability of noncompliance (PNC) values between the limit and the design inputs were then generated (i.e. demand exceeding supply). The main contribution of this study is that by applying the racing car model, design problems could be detected at roads before construction.

A micro simulation technique, Monte Carlo Simulation (MCS), was used in a study to account for how SCL vehicles accelerate to their merging speed as well as searching for and finding an acceptable gap in the freeway through traffic (Fatema \& Hassan, 2013). MCS, as will be explained Section 5.4, was used to account for the variability in driver's speed, acceleration, and gap acceptance characteristics based on field data. The model runs for every SCL using its SCL available length to produce the probability of uncomfortable merging, PNC. The results of the authors' study are summarized earlier in Section 2.3. Fatema \& Hassan's (2013) MCS model was later 
improved to cover sixteen limited-length SCLs and seven extended-length SCLs (Fatema, Ismail \& Hassan, 2014). The model finds the probability of failed maneuvers by simulating vehicles acceleration and gap acceptance assuming that:

- Drivers accelerate from the end of controlling curve up to the point where the pavement edges of the FRL and ramp are $1.25 \mathrm{~m}$ apart.

- After that point, drivers accelerate and look for an acceptable up to the end of the SCL.

If drivers reach an acceptable merging speed and an acceptable gap is available on the freeway, a successful merging takes place. In case any of the two criteria is violated a forced merging takes place and is referred to as a failed merging. Using negative binomial regression, Fatema, Ismail \& Hassan (2014) showed that including PNC in the regression model showed the best fitted model. Their study is quantitative evidence supporting the probabilistic approach to study safety.

Sarhan \& Hassan (2011) argued that the deterministic approach used by the design guides to design for the lateral clearance on horizontal curves is insufficient and may over and/or under-estimate the available sight distance for road users. They developed a new method to calculate the lateral clearance on horizontal curves considering the effect of vertical curvature on the road alignment. A probabilistic approach was used to evaluate the probability of demand sight distance to exceed supply sight distance (called probability of hazard). Variations within the distribution of the following parameters were taken into account when designing: speed, perception and reaction time, deceleration rate, friction coefficient, and driver eye and object heights. Using a selected probability of hazard, a designer can estimate the lateral clearance and 
the height at which the lateral clearance is provided. Finally, an example design aid was created for $400 \mathrm{~m}$ horizontal curves with a side slop 2:1 (horizontal: vertical) (Sarhan \& Hassan, 2011). This study provides a probabilistic technique for design, which could help in granting consistency in the design of road elements in a particular area.

Hassan, Sarhan and Salehi developed probabilistic models to design for freeway acceleration SCLs (Hassan, Sarhan \& Salehi, 2012). Limitation of one of the methods was pointed out, which is generating a high standard deviation of the estimated length than the data when using First Order Second Moment (FOSM) method. They found that recommended SCL lengths is nearly constant for curves of design speeds between 50 to $80 \mathrm{~km} / \mathrm{h}$ as drivers use lower acceleration rates when entering the SCL with high speeds. Recommended SCLs were compared to the Canadian design guide (TAC 1999) and the Green Book (AASHTO 2004). It was found that the recommended design lengths using FOSM method fall within the TAC (1999) recommended lengths range but not with the entire Green Book's values.

Felipe (1996) used First Order Reliability (FORM) Analysis to find the PNC between the demanded radius of horizontal curves by the car/driver system and the supplied radius of the horizontal curve of the road. The data collected for this research were speed, lateral acceleration, and level of comfort. The study found that as the radius of horizontal curves decreases, PNC increases (Felipe, 1996).

PNC values in the literature are usually associated with one mode of failure which is demand exceeding supply. But in a reliability based risk analysis of horizontal curves, You, Sun \& Gu (2012) developed two failure types, where each type has its own performance function. The first failure mode is skidding without rolling, and the second 
is skidding and rolling (You, Sun \& Gu, 2012). They also verified that AASHTO's minimum recommended radius to prevent skidding provides enough safety margins for vehicles in dry conditions. However, minimum radius values were questioned for trucks in wet pavement conditions.

Shin \& Lee (2013) used reliability analysis to test the PNC of current horizontal curves for trucks. Two failure modes were tested, which are rollover and sideslip (Shin \& Lee, 2013). Using FORM, the study found that rollover is dependent mostly on the vehicle's speed and the steering angle, while sideslip depends on the tire road friction coefficient. The study found that both types of accidents could be reduced by increasing the superelevation rate. The authors found that the current minimum radius values recommended in AASHTO could not ensure sufficient margin of safety in both rollover and sideslip conditions.

\subsection{Summary}

Many researchers studied safety and operations on highway elements such as deceleration SCLs. Many studies focused on the relationship between geometric configuration and conditions of highway elements and safety. Some of the topics studied are: the effect of SCL length choices on safety; different ramps' configuration choices on safety; and road conditions in terms of sight distance or surface and weather conditions. Part of these studies used simulation techniques quantify or address possible issues in the drivers' performance while travelling on different highway elements. Other studies used probabilistic approaches in order to quantify the difference between what the driver needs in terms of geometry and what is supplied on the actual highway segment or 
recommended by the design guides. However, fewer studies linked operations and safety. There is a likely strong relation between safety and operations on highway segments and more research is needed in this topic. The main purpose of this study is to investigate operations on deceleration SCLs using three different reliability approaches and to develop a design aid for practitioners to be able to design SCLs based on the required level of operational performance. The measure developed in this study could be used for future research as a surrogate safety measure to explicitly study safety. 


\section{Chapter Three}

\subsection{Data Description}

The study sites used in this research are deceleration SCLs on Highway 417 in Ottawa, Ontario. As this research is continuity to previous research done by El-Basha (2006), the same data will be used in this thesis. The data included drivers speed profiles and geometry of the selected study sites. However, these data were re-processed for the purpose of performing reliability analysis of deceleration SCLs. In addition, to explore the premise of a relationship between the developed models and safety, collision data at the study sites were collected (Sarhan, 2004).

This Chapter is organized in the following order:

- Study area and selected sites.

- Geometric characteristics of the study sites.

- Available speed data collected by El-Basha (2006).

- Data reprocessing for the purpose of this research.

- Collision data.

\subsection{Study Area and Selected Sites}

El-Basha (2006) collected data from 13 exit ramp terminals at 11 interchanges on Highway 417. The study sites included both limited and extended SCLs. The study site selection was limited to accessible sites with a number of exiting vehicles to 
allow good sample size in one hour of data collection. Selection of study sites was controlled by:

$>$ Visibility: Locations with limited visibility at the SCL or the Freeway Right Lane (FRL) were excluded.

$>$ Drivers' distraction: Locations where the operator would be visible to drivers were excluded.

Safety: Locations with safety hazards to the operators were excluded.

It should be noted that out of 40 candidate exit terminals to collect the speed profiles, 27 sites where excluded because of not meeting the above criteria. Figure 3.1 shows a Google Earth map with the locations of eleven interchanges containing the thirteen SCL sites where data was collection.

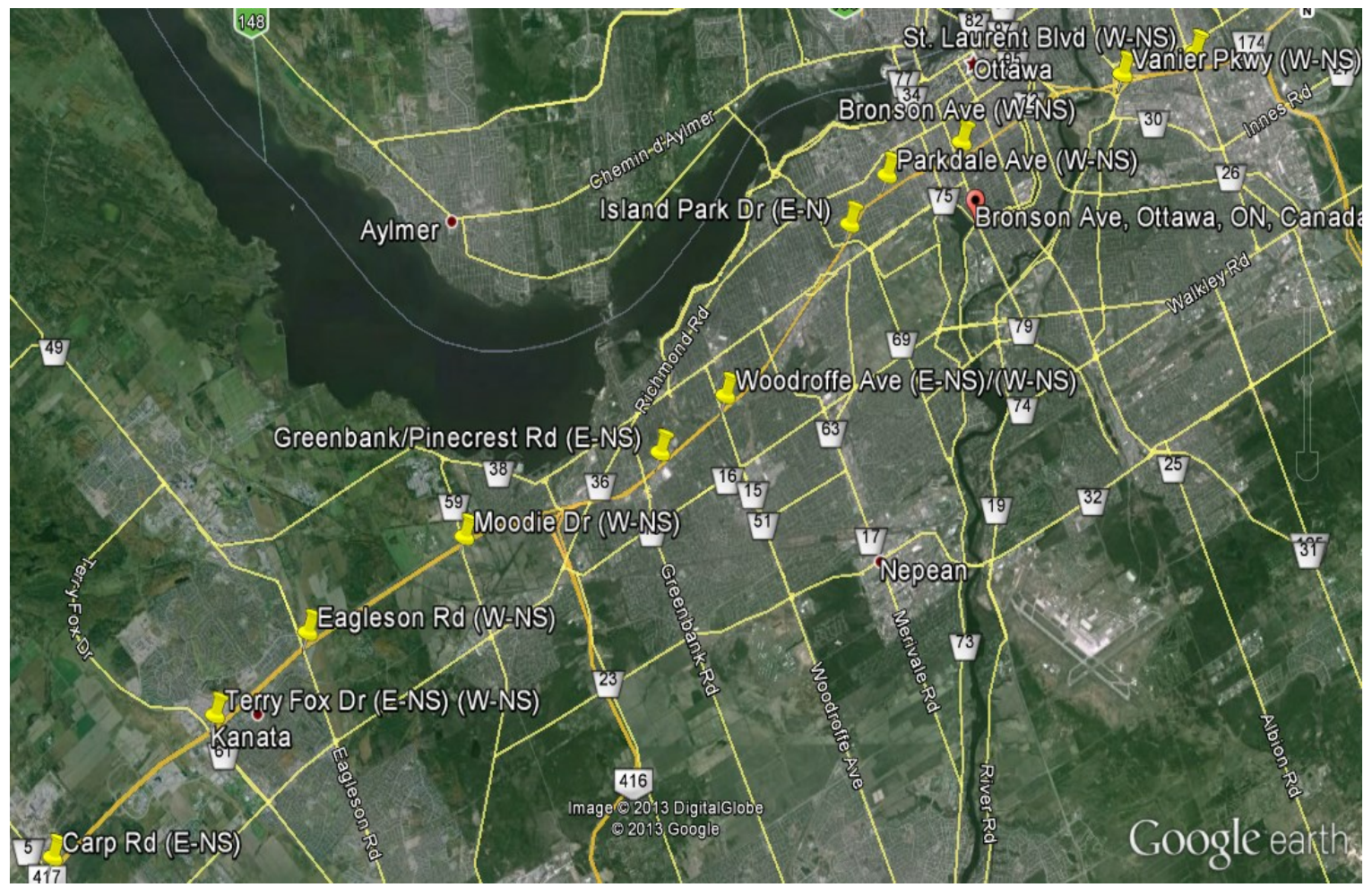

Figure 3.1: Study Area (Google Earth Imagery) 
Data for the all selected SCLs were collected from interchange overpasses except for Bronson W-NS, which speed data were collected from the roof of an Ottawa-Carleton School Board building located next to Highway 417. Table 3.1 is a summary of the study sites SCLs.

Table 3.1: Selected Sites

\begin{tabular}{|l|l|l|l|}
\hline No. & SCL Name & Type & $\begin{array}{c}\text { Actual Length } \\
(\mathrm{m})\end{array}$ \\
\hline 1 & Bronson Avenue (W-NS) & Limited & 212 \\
\hline 2 & Carp Road (E-NS) & Limited & 430 \\
\hline 3 & Moodie Drive (W-NS) & Limited & 292 \\
\hline 4 & Parkdale Avenue (W-NS) & Limited & 179 \\
\hline 5 & Island Park Drive (E-N) & Limited & 58 \\
\hline 6 & Terry Fox Drive (E-NS) & Limited & 388 \\
\hline 7 & Terry Fox Drive(W-NS) & Limited & 446 \\
\hline 8 & Greenbank/Pinecrest Road (E-NS) & Extended (Dual Exit) & 1047 \\
\hline 9 & St. Laurent Blvd (W-NS) & Extended (Dual Exit) & 873 \\
\hline 10 & Vanier Pkwy (W-NS) & Extended (Dual Exit) & 707 \\
\hline 11 & Eagleson Road (W-NS) & Extended (Single Exit) & 697 \\
\hline 12 & Woodroffe Avenue (E-NS) & Extended (Single Exit) & 1320 \\
\hline 13 & Woodroffe Avenue (W-NS) & Extended (Single Exit) & 1358 \\
\hline
\end{tabular}

SCL length includes the taper for limited-length SCL and is measured to the gore area where pavement edges of the freeway right lane and off-ramp are separated by $1.25 \mathrm{~m}$.

As mentioned in Chapter 1.0, extended SCLs, which are extended from an entrance ramp to an exit ramp, are affected by both merging and diverging vehicles and the associated weaving manoeuvres and are beyond the scope of this research. Therefore, only data related to the seven limited-length SCLs were included for further analysis. 


\subsection{Geometric Characteristics of the Study Sites}

El-Basha (2006) obtained the geometric planes of the study sites from Ontario Ministry of Transportation (MTO), Engineering and Title Records (ETR). Plans were validated through field visits to the study sites by the data collection team. The geometric information collected in El-Basha's (2006) thesis included: lengths of deceleration SCLs, transition lengths (from end of SCLs to controlling ramp curves), divergence angle at the gore area, ramp width, lengths of freeway segments, and number of freeway basic lanes. More details on how the geometric data were determined could be found in El-Basha (2006). A summary of the geometric data of the study sites used in this research is available in Appendix 1.

\subsection{Available Speed Data}

El-Basha (2006) collected 1,524 speed profiles on limited-length SCLs and adjacent freeway right lanes (FRLs) at the seven study sites. Processed data included initial/diverge and final/gore speeds, and the deceleration time and distance. The data were collected using laser guns that track individual vehicles from the diverge point onto the deceleration SCL up to the gore area every 0.05 seconds. In the original data processing by El-Basha (2006), both speeds and distances were corrected for the cosine error due to the equipment's positioning.

Using the readings collected by the laser guns, the speed profiles were processed by El-Basha (2006). Diverge speed, maximum speed, mean speed, gore speed, maximum deceleration rate, mean deceleration rate, overall deceleration rate, and total travelled distance on SCL were estimated for every vehicle. Diverge speed was determined as the first corrected speed data reading of the targeted vehicle as it changes lane form FRL to 
SCL, while gore speed is the last speed data reading recorded at the proximity of the gore area. The maximum speed is the maximum value reached by a targeted vehicle on the SCL of all the successive corrected speed readings along the speed profile. Mean speed is the average of all the successive speed data readings of the corrected speed profile. Maximum deceleration rate was calculated as the minimum negative acceleration value of all the successive acceleration rates. Mean deceleration rate was calculated as the average of all successive deceleration rates. Also, the overall deceleration rate was calculated as the average deceleration rate based on the overall distance travelled on the SCL and the overall time elapsed while decelerating. Overall deceleration rate considers the entire vehicle's profile as one interval as shown in Equations 3.1 and 3.2 (El-Basha, 2006). Finally, the total travelled distance on SCL is the total distance travelled on the deceleration lane from the diverge point till the end of the deceleration SCL.

$\boldsymbol{d}_{\text {Time }}=\frac{v_{f}-v_{i}}{T}$

(Equation 3.3)

$d_{D i s}=\frac{v_{f}{ }^{2}-v_{i}{ }^{2}}{2 * D}$

(Equation 3.4)

Where,

- $d_{\text {Time }}$ is the overall deceleration rate for the speed profile based on its time interval $\left(\mathrm{m} / \mathrm{sec}^{2}\right)$.

- $d_{D i s}$ is the overall deceleration rate for the speed profile based on its distance interval $\left(\mathrm{m} / \mathrm{sec}^{2}\right)$.

- $v_{f}$ is the final speed at the speed profile $(\mathrm{m} / \mathrm{sec})$.

- $v_{i}$ is the initial speed at the first data point on the speed profile $(\mathrm{m} / \mathrm{sec})$.

- $T$ is the travel time interval in the speed profile first and last data points (sec).

- $D$ is the distance interval in the speed profile's first and last data points (m). 
Figures 3.2 and 3.3 show screen shots of a speed profile sample and the final speed profile's summary, respectively, on Bronson Avenue (W-NS) SCL.

Diverge speeds, gore speeds, maximum speeds, mean speeds, overall deceleration rates, maximum deceleration rates, mean deceleration rates, and total travelled distances for all vehicles on each SCL were summarized as shown in Figure 3.3. In the data processed by El-Basha (2006), each targeted vehicle was categorized as follows:

- "D": a vehicle that diverged from right lane to SCL.

- "RL": a vehicle that continued through on the right-lane.

- "Sh": vehicles with short speed profiles, where the last speed reading did not correspond to the gore area.

- "out": a corrupted file.

- "HV": a heavy vehicle.

Speed profiles marked as "Sh" and were not considered for any further analysis in this research as it could be misleading as the last speed reading does not represent the gore speed. 


\begin{tabular}{|c|c|c|c|c|c|c|c|c|c|c|c|c|c|c|c|c|c|c|c|}
\hline & $A$ & B & C & D & $E$ & $F$ & G & $H$ & 1 & $J$ & K & L & M & N & 0 & $P$ & Q & $R$ & S \\
\hline 2 & $d(m)$ & Corr. d (m) & d Inter. & d scale & $\mathrm{s}(\mathrm{km} / \mathrm{h})$ & Corr. s (km/h) & $\mathrm{s}(\mathrm{m} / \mathrm{sec})$ & $\mathrm{t}(\mathrm{hr})$ & $\mathrm{t}(\mathrm{sec})$ & t Inter & t scale & Dec. $\mathrm{t}\left(\mathrm{m} / \mathrm{s}^{\wedge} \mathrm{2}\right)$ & Dec. $d\left(\mathrm{~m} / \mathrm{s}^{\wedge} 2\right)$ & Av. Dec. & & & & & \\
\hline 3 & 289 & 288.734715 & & 0 & 86.5 & 86.57947489 & 24.0498541 & $20: 32.6$ & & & 0 & & & & & \multirow{2}{*}{\multicolumn{4}{|c|}{ Average Deceleration time $=-1.16921682$}} \\
\hline 4 & 264 & 263.709567 & 25.0251482 & 25.0251482 & 81.2 & 81.28942861 & 22.5803968 & $20: 33.7$ & $00: 01.11$ & 1.11 & 1.11 & -1.323835406 & -1.369046095 & -1.34644075 & & & & & \\
\hline 5 & 262.87 & 262.578317 & 1.13124987 & 26.156398 & 81.2 & 81.2902004 & 22.5806112 & $20: 33.8$ & $00: 00.05$ & 0.05 & 1.16 & 0.004287727 & 0.004279295 & 0.004283511 & & & & & \\
\hline 6 & 261.72 & 261.427034 & 1.15128309 & 27.3076811 & 81.2 & 81.29099616 & 22.5808323 & $20: 33.8$ & $00: 00.05$ & 0.05 & 1.21 & 0.00442089 & 0.004335462 & 0.004378176 & & & & & \\
\hline 7 & 260.62 & 260.325796 & 1.10123792 & 28.408919 & 81.2 & 81.29176722 & 22.5810465 & $20: 33.9$ & $00: 00.06$ & 0.06 & 1.27 & 0.003569732 & 0.004391853 & 0.003980793 & & & & & \\
\hline 8 & 258.37 & 258.073231 & 2.25256499 & 30.661484 & 81 & 81.09314526 & 22.5258737 & $20: 34.0$ & $00: 00.10$ & 0.1 & 1.37 & -0.551727667 & -0.552408829 & -0.55206825 & & & & & \\
\hline 9 & 257.25 & 256.951937 & 1.12129355 & 31.7827776 & 80.9 & 80.9938435 & 22.4982899 & $20: 34.0$ & $00: 00.05$ & 0.05 & 1.42 & -0.551676434 & -0.553797219 & -0.55273683 & & & & & \\
\hline 10 & 256.14 & 255.840644 & 1.11129318 & 32.8940708 & 80.8 & 80.89454304 & 22.4707064 & $20: 34.1$ & $00: 00.05$ & 0.05 & 1.47 & -0.551669227 & -0.558088806 & -0.55487902 & & & & & \\
\hline 11 & 255.04 & 254.739351 & 1.10129266 & 33.9953634 & 80.7 & 80.79524376 & 22.4431233 & $20: 34.1$ & $00: 00.05$ & 0.05 & 1.52 & -0.551662657 & -0.56245909 & -0.55706087 & & \multicolumn{4}{|c|}{ Max. Decceleration $=-1.77233852$} \\
\hline 12 & 253.96 & 253.658071 & 1.08128007 & 35.0766435 & 80.6 & 80.69593798 & 22.4155383 & $20: 34.2$ & $00: 00.05$ & 0.05 & 1.57 & -0.551698793 & -0.572203035 & -0.56195091 & & \multicolumn{4}{|c|}{ Mean Deceleration $=-0.97822865$} \\
\hline 13 & 252.9 & 252.596804 & 1.06126701 & 36.1379105 & 80.4 & 80.49650528 & 22.3601404 & $20: 34.2$ & $00: 00.05$ & 0.05 & 1.62 & -1.107959449 & -1.168641719 & -1.13830058 & & \multicolumn{3}{|c|}{ Overall Average Deceleration } & -1.18849371 \\
\hline 14 & 251.83 & 251.525515 & 1.0712898 & 37.2092003 & 80.1 & 80.19696543 & 22.2769348 & $20: 34.3$ & $00: 00.05$ & 0.05 & 1.67 & -1.664110273 & -1.733448203 & -1.69877924 & & \multicolumn{4}{|c|}{\begin{tabular}{l|l} 
& Diverging Speed $=86.57947489$
\end{tabular}} \\
\hline 15 & 250.76 & 250.454214 & 1.07130083 & 38.2805011 & 79.8 & 79.89742995 & 22.1937305 & $20: 34.3$ & $00: 00.05$ & 0.05 & 1.72 & -1.664086026 & -1.72694286 & -1.69551444 & & \multicolumn{3}{|c|}{ Maximum Speed } & 86.57947489 \\
\hline 16 & 245.46 & 245.147603 & 5.3066109 & 43.587112 & 78.2 & 78.29965202 & 21.7499033 & $20: 34.6$ & $00: 00.26$ & 0.26 & 1.98 & -1.707027695 & -1.83764934 & -1.77233852 & & \multicolumn{3}{|c|}{ Mean Speed $=$} & 78.20715408 \\
\hline 17 & 244.39 & 244.076233 & 1.07136951 & 44.6584815 & 77.9 & 78.00014256 & 21.6667063 & $20: 34.6$ & $00: 00.05$ & 0.05 & 2.03 & -1.663941435 & -1.685755831 & -1.67484863 & & \multicolumn{3}{|c|}{ End Operating Speed } & 69.82693636 \\
\hline 18 & 243.35 & 243.034891 & 1.04134267 & 45.6998242 & 77.7 & 77.8007427 & 21.6113174 & $20: 34.7$ & $00: 00.05$ & 0.05 & 2.08 & -1.107776997 & -1.150975571 & -1.12937628 & & \multicolumn{3}{|c|}{ Deceleration Distance } & 83.6981238 \\
\hline 19 & 242.29 & 241.97351 & 1.06138038 & 46.7612046 & 77.5 & 77.60136626 & 21.5559351 & $20: 34.7$ & $00: 00.05$ & 0.05 & 2.13 & -1.107646917 & -1.126223811 & -1.11693536 & & \multicolumn{3}{|c|}{ Time elapsed on SCL } & 3.98 \\
\hline 20 & 241.23 & 240.912118 & 1.06139253 & 47.8225971 & 77.3 & 77.40199692 & 21.5005547 & $20: 34.8$ & $00: 00.05$ & 0.05 & 2.18 & -1.10760743 & -1.123281126 & -1.11544428 & & \multicolumn{3}{|c|}{ Diverging Point } & 128.2652851 \\
\hline 21 & 240.18 & 239.860726 & 1.05139154 & 48.8739887 & 77.1 & 77.20262625 & 21.445174 & $20: 34.8$ & $00: 00.05$ & 0.05 & 2.23 & -1.107614873 & -1.131056463 & -1.11933567 & & & & & \\
\hline 22 & 239.14 & 238.819336 & 1.04139036 & 49.915379 & 76.9 & 77.00325407 & 21.3897928 & $20: 34.9$ & $00: 00.05$ & 0.05 & 2.28 & -1.107623176 & -1.138982171 & -1.12330267 & & & & & \\
\hline 23 & 238.09 & 237.76792 & 1.05141607 & \begin{tabular}{|l|}
50.9667951 \\
\end{tabular} & 76.7 & 76.80389774 & 21.334416 & $20: 34.9$ & $00: 00.05$ & 0.05 & 2.33 & -1.107535196 & -1.125115126 & -1.11632516 & & & & & \\
\hline 24 & 237.03 & 236.706478 & 1.06144231 & 52.0282374 & 76.5 & 76.60455764 & 21.2790438 & $20: 35.0$ & $00: 00.05$ & 0.05 & 2.38 & -1.107445012 & -1.111507972 & -1.10947649 & & & & & \\
\hline 25 & 235.98 & 235.655036 & 1.0514415 & 53.0796789 & 76.4 & 76.5053542 & \begin{tabular}{|l|}
21.2514873 \\
\end{tabular} & $20: 35.0$ & $00: 00.05$ & 0.05 & 2.43 & -0.551130187 & -0.557326762 & -0.55422847 & & & & & \\
\hline 26 & 234.94 & 234.613596 & 1.0414405 & 54.1211194 & 76.3 & 76.40615183 & 21.2239311 & $20: 35.1$ & $00: 00.05$ & 0.05 & 2.48 & -0.551124296 & -0.561943648 & -0.55653397 & & & & & \\
\hline 27 & 233.91 & 233.582156 & 1.0314393 & 55.1525587 & 76.2 & 76.30695036 & 21.1963751 & $20: 35.1$ & $00: 00.05$ & 0.05 & 2.53 & -0.551119247 & -0.566651065 & -0.55888516 & & & & & \\
\hline 28 & 232.88 & 232.550704 & 1.03145206 & 56.1840108 & 76 & 76.10761733 & 21.1410048 & $20: 35.2$ & $00: 00.05$ & 0.05 & 2.58 & -1.107405775 & -1.136375134 & -1.12189045 & & & & & \\
\hline 29 & 231.86 & 231.529253 & 1.02145071 & 57.2054615 & 75.8 & 75.9082826 & 21.0856341 & $20: 35.2$ & $00: 00.05$ & 0.05 & 2.63 & -1.107415152 & -1.144509947 & -1.12596255 & & & & & \\
\hline 30 & 230.92 & 230.587905 & 0.9413483 & 58.1468098 & 75.6 & 75.70887983 & \begin{tabular}{|l|}
21.0302444 \\
\end{tabular} & $20: 35.3$ & $00: 00.05$ & 0.05 & 2.68 & -1.107793178 & -1.239065365 & -1.17342927 & & & & & \\
\hline 31 & 229.81 & 229.476299 & 1.11160637 & 59.2584161 & 75.4 & 75.50964563 & 20.9749016 & $20: 35.3$ & $00: 00.05$ & 0.05 & 2.73 & -1.106056660 & -1.04564163 & -1.07624915 & & & & & \\
\hline 32 & 228.81 & 228.474838 & 1.00146056 & 60.2598767 & 75.1 & 75.21016815 & 20.8917134 & $20: 35.4$ & $00: 00.05$ & 0.05 & 2.78 & -1.663763767 & -1.7388664217 & -1.70131399 & & & & & \\
\hline 32 & $\longdiv { 2 2 7 8 6 }$ & 227523139 & ח 95139913 & 612112761 & 719 & $75 \cap 1070180$ & $2 n 8363319$ & $2 n \cdot 35.1$ & $n \cap \cap \cap \cap \cap 5$ & $\cap \cap \cap 5$ & 283 & $.11 \cap 76292 n 5$ & $.12115 \Omega 5712$ & $.1161 \cap G 71 h$ & & & & & \\
\hline
\end{tabular}

Figure 3.2: Speed Profile Sample for a passenger car on Bronson Ave. W-NS 


\begin{tabular}{|c|c|c|c|c|c|c|c|c|c|c|c|c|c|c|c|}
\hline 2 & Vehicle Type & Data File no. & Behaviour & Max. Deceleration & Mean Deceleration & Overall Deceleration & Diverg Speed & Maximum Speed & Mean Speed & End Operating Speed & Travelled Distance & Time Elapsed & Diverging Point & Distance Left (1.25) & Deceleration Distance \\
\hline 3 & HV & 29 & Sh & -2.257885561 & -0.987927675 & -0.99183632 & 77.75961806 & 77.75961806 & 67.3818696 & 61.4913497 & 85.50125227 & 4.70 & 113.79 & 12.71 & 98.20735347 \\
\hline 4 & HV & 43 & & -2.284599074 & -0.720272982 & -0.793127699 & 91.07371013 & 93.28503891 & 88.2809648 & 80.23486778 & 90.46552122 & 3.79 & 121.8990322 & 0 & 90.46552122 \\
\hline 5 & HV & 48 & & -2.288770612 & -1.025568227 & -1.0204105 & 76.35401186 & 76.35401186 & 66.4161503 & 53.49918029 & 110.6003505 & 6.313301357 & 101.3234637 & 0 & 110.6003505 \\
\hline 6 & HV & 71 & & -2.279717309 & -0.837488971 & -1.175097823 & 89.56020974 & 89.76082812 & 84.0282272 & 66.51406828 & 118.7459678 & 5.417969304 & 93.17784634 & 0 & 118.7459678 \\
\hline 7 & HV & 73 & & -2.288233323 & -0.631717282 & -0.881001165 & 80.55695972 & 80.76187092 & 75.0851199 & 62.88256842 & 110.6704 & 5.590365127 & 101.2534141 & 0 & 110.6704 \\
\hline 8 & HV & 102 & & -2.350104906 & -0.987165437 & -1.685772495 & 82.54808708 & 82.54808708 & 72.0658872 & 24.24037448 & 142.3201978 & 9.62 & 69.10278012 & 0 & 142.3201978 \\
\hline 9 & PC & 1 & & -2.249812435 & -0.682831321 & -1.065069848 & 82.98266243 & 83.18497746 & 78.3014408 & 69.72740022 & 72.6379989 & 3.49 & 139.8463594 & 0 & 72.6379989 \\
\hline 10 & $P C$ & 2 & & -1.708708312 & -0.487729976 & -0.494447592 & 88.47812088 & 88.48009544 & 84.3815309 & 81.64883289 & 89.65374356 & 3.88 & 122.5901759 & 0 & 89.65374356 \\
\hline 11 & PC & 3 & & -2.272070227 & -0.863392568 & -1.88733033 & 82.99715975 & 83.10090107 & 78.7471138 & 65.92015027 & 50.86429947 & 2.57 & 161.3696018 & 0 & 50.86429947 \\
\hline 12 & $P C$ & 4 & & -2.281862524 & -0.862179079 & -0.938841594 & 93.67715568 & 93.67715568 & 84.5724934 & 79.34399849 & 99.63181705 & 4.34 & 112.1612825 & 0 & 99.63181705 \\
\hline 13 & PC & 5 & & -2.809248688 & -0.724576432 & -1.147822466 & 81.47992706 & 81.58408973 & 74.3178091 & 66.30449319 & 74.14896677 & 3.734553591 & 137.7042419 & 0 & 74.14896677 \\
\hline 14 & $P C$ & 6 & & -2.84006744 & -0.916272981 & -1.40943084 & 91.88549589 & 91.88549589 & 82.0766419 & 73.5336095 & 81.69622894 & 3.68 & 130.2170889 & 0 & 81.69622894 \\
\hline 15 & $\mathrm{PC}$ & 7 & & -1.672675187 & -0.586117431 & -0.622332303 & 92.87268819 & 93.27487225 & 90.4751282 & 82.97937885 & 107.6884582 & 4.422627098 & 104.2749506 & 0 & 107.6884582 \\
\hline 16 & PC & 8 & & -2.287514933 & -0.351600316 & -0.449072396 & 75.36100903 & 75.46324015 & 71.4766865 & 66.92180949 & 102.5745208 & 5.25 & 109.519125 & 0 & 102.5745208 \\
\hline 17 & PC & 9 & & -1.772338518 & -0.978228652 & -1.188493712 & 86.57947489 & 86.57947489 & 78.2071541 & 69.82693636 & 83.6981238 & 3.98 & 128.2652851 & 0 & 83.6981238 \\
\hline 18 & PC & 10 & & -1.241941528 & -0.379043479 & -0.803838772 & 89.67173364 & 92.18139617 & 86.6667491 & 76.58684858 & 104.2857658 & 4.527141827 & 107.6776431 & 0 & 104.2857658 \\
\hline 19 & PC & 11 & & -2.279466901 & -1.240591234 & -1.43478007 & 85.9833158 & 85.9833158 & 73.5121711 & 67.1221026 & 76.05097305 & 3.73 & 135.9825634 & 0 & 76.05097305 \\
\hline 20 & $P C$ & 12 & & -2.289783624 & -0.729973262 & -0.806249267 & 87.37736947 & 87.37802125 & 82.060606 & 75.93749464 & 88.54211604 & 3.98 & 123.0105476 & 0 & 88.54211604 \\
\hline 21 & PC & 13 & & -2.290680319 & -1.403723128 & -1.159734837 & 89.28197265 & 89.28197265 & 83.1625013 & 73.93385377 & 83.2473329 & 3.68 & 128.2953126 & 0 & 83.2473329 \\
\hline 22 & $P C$ & 14 & & -2.792607165 & -0.715763307 & -0.842142986 & 89.45688578 & 89.45729469 & 81.8479241 & 70.12769457 & 142.1129337 & 6.34 & 70.02078506 & 0 & 142.1129337 \\
\hline 23 & PC & 15 & & -2.291669397 & -1.140447597 & -1.244761807 & 92.57177657 & 92.57236522 & 84.6276488 & 70.92901523 & 109.2197136 & 4.85 & 102.8038046 & 0 & 109.2197136 \\
\hline 24 & PC & 16 & & -2.563771064 & -0.842593457 & -1.083994165 & 83.1763616 & 83.1763616 & 73.3619883 & 67.22207015 & 83.5679052 & 4.18 & 128.2853035 & 0 & 83.5679052 \\
\hline 25 & PC & 17 & & -2.475423261 & -0.721099183 & -1.081963713 & 91.68027089 & 91.68027089 & 81.5584935 & 76.33895034 & 90.74456105 & 3.99 & 121.3490847 & 0 & 90.74456105 \\
\hline 26 & PC & 18 & & -2.227864387 & -1.115290883 & -1.315610295 & 90.58478726 & 90.58478726 & 87.9034705 & 73.55406499 & 80.88550778 & 3.644967048 & 131.0678829 & 0 & 80.88550778 \\
\hline 27 & PC & 19 & Sh & -1.694707777 & -0.584634845 & -0.576770422 & 94.08022075 & 94.38263695 & 92.4229087 & 90.0101835 & 49.62069593 & 1.98 & 117.4056775 & 44.97362661 & 94.59432254 \\
\hline 28 & $P C$ & 20 & & -1.943470898 & -1.364873659 & -1.377577323 & 93.69030127 & 93.69030127 & 83.8543039 & 77.44096996 & 76.87182616 & 3.32 & 135.2318379 & 0 & 76.87182616 \\
\hline 29 & PC & 21 & & -2.265597373 & -0.976966606 & -1.175602127 & 94.47050397 & 94.47050397 & 83.7597817 & 73.40621096 & 115.2141994 & 5.01352073 & 96.73919128 & 0 & 115.2141994 \\
\hline 30 & PC & 22 & & -1.566194629 & 0.640420101 & -0.779197845 & 84.17757055 & 88.09788836 & 85.8304191 & 73.68319874 & 83.12759006 & 3.692200714 & 128.8258006 & 0 & 83.12759006 \\
\hline 31 & $P C$ & 23 & Sh & -1.714201058 & -0.96241603 & -0.620315814 & 87.06789301 & 87.17982136 & 84.2968135 & 78.31976025 & 89.69794906 & \begin{tabular}{|l|}
.93 \\
\end{tabular} & 103.6944972 & 18.60755376 & 108.3055028 \\
\hline 32 & PC & 24 & & -1.416057078 & -0.225567671 & -0.70809398 & 80.36579012 & 80.87193652 & 79.6797651 & 67.82671303 & 100.7228725 & 4.94449071 & 111.2305182 & 0 & 100.7228725 \\
\hline 33 & PC & 25 & & -2.298518791 & -0.644343602 & -1.277166053 & 87.7860514 & 87.7860514 & 83.3685045 & 72.33146364 & 74.33922872 & 3.38 & 137.6041437 & 0 & 74.33922872 \\
\hline 34 & PC & 26 & & -2.275516255 & -1.106005606 & -1.187434275 & 98.48632916 & 98.48632916 & 95.3804458 & 83.03362156 & 90.43415687 & 3.643094668 & 121.5192338 & 0 & 90.43415687 \\
\hline 35 & PC & 27 & & -2.289988613 & -0.659860124 & -0.668420982 & 84.3597969 & 84.3597969 & 79.1100824 & 70.52823623 & 123.6103389 & 5.75 & 88.3731064 & 0 & 123.6103389 \\
\hline 36 & PC & 28 & & -2.791981744 & -0.996836494 & -1.264544224 & 95.08573289 & 95.08573289 & 87.1466841 & 78.54280852 & 86.32052773 & 3.69 & 125.6629176 & 0 & 86.32052773 \\
\hline
\end{tabular}

Figure 3.3: Summary of Speed Profiles on Bronson Ave. W-NS 


\subsection{Data Reprocessing}

Data of SCL vehicles were initially summarized for the purpose of this research to include diverge speed, end operating/gore speed, overall deceleration, and total travelled distance. Figure 3.4 shows the new order of data.

\begin{tabular}{|c|c|c|c|c|c|}
\hline 1 & Vehicle Ty & Diverg Speed & End Operating Speed & Overall Deceleration & Total Travelled Distance \\
\hline 2 & HV & 21.59989391 & 17.08093047 & 0.99183632 & 98.20735347 \\
\hline 3 & HV & 25.29825281 & 22.28746327 & 0.793127699 & 90.46552122 \\
\hline 4 & $\mathrm{HV}$ & 21.20944774 & 14.86088341 & 1.0204105 & 110.6003505 \\
\hline 5 & HV & 24.87783604 & 18.47613008 & 1.175097823 & 118.7459678 \\
\hline 6 & HV & 22.37693325 & 17.46738012 & 0.881001165 & 110.6704 \\
\hline 7 & $\mathrm{HV}$ & 22.93002419 & 6.733437357 & 1.685772495 & 142.3201978 \\
\hline 8 & PC & 23.05073956 & 19.36872228 & 1.065069848 & 72.6379989 \\
\hline 9 & PC & 24.5772558 & 22.68023136 & 0.494447592 & 89.65374356 \\
\hline 10 & PC & 23.0547666 & 18.31115285 & 1.88733033 & 50.86429947 \\
\hline 11 & PC & 26.02143213 & 22.03999958 & 0.938841594 & 99.63181705 \\
\hline 12 & PC & 22.63331307 & 18.41791478 & 1.147822466 & 74.14896677 \\
\hline 13 & PC & 25.52374886 & 20.42600264 & 1.40943084 & 81.69622894 \\
\hline 14 & PC & 25.79796894 & 23.04982746 & 0.622332303 & 107.6884582 \\
\hline 15 & PC & 20.93361362 & 18.58939152 & 0.449072396 & 102.5745208 \\
\hline 16 & PC & 24.04985414 & 19.39637121 & 1.188493712 & 83.6981238 \\
\hline 17 & PC & 24.9088149 & 21.2741246 & 0.803838772 & 104.2857658 \\
\hline 18 & PC & 23.88425439 & 18.6450285 & 1.43478007 & 76.05097305 \\
\hline 19 & PC & 24.27149152 & 21.09374851 & 0.806249267 & 88.54211604 \\
\hline 20 & PC & 24.80054796 & 20.5371816 & 1.159734837 & 83.2473329 \\
\hline 21 & PC & 24.84913494 & 19.47991516 & 0.842142986 & 142.1129337 \\
\hline 22 & PC & 25.71438238 & 19.70250423 & 1.244761807 & 109.2197136 \\
\hline 23 & PC & 23.10454489 & 18.67279727 & 1.083994165 & 83.5679052 \\
\hline 24 & PC & 25.46674191 & 21.20526398 & 1.081963713 & 90.74456105 \\
\hline 25 & PC & 25.16244091 & 20.43168472 & 1.315610295 & 80.88550778 \\
\hline
\end{tabular}

\section{Figure 3.4: Initial Data Organization (Speeds are in $\mathrm{m} / \mathrm{s}$, Deceleration in $\mathrm{m} / \mathrm{s}^{2}$, and distance in $\mathrm{m}$ )}

Deceleration behaviour on limited SCLs is mainly affected by drivers' choice of initial speed, available distance on SCL, and final speed based on the end conditions of the exit ramp. For example, a sharp controlling curve on the exit ramp will force drivers to reach a low the final speed. El-Basha (2006) used the total travelled distance on SCLs to model drivers' deceleration behavior. However, in many cases, such an assumption might not represent the real conditions. If the SCL is long enough, drivers might maintain 
their speed, accelerate, or decelerate slightly. To be able to produce more sound conclusions on the operational performance of the SCLs, the real deceleration point should be investigated. In case deceleration does not occur at the diverge point for a specific vehicle, new deceleration distance, overall deceleration rate and initial speed should be found.

Assuming that drivers decelerate at a constant rate, a MATLAB script (Appendix 2) was developed to estimate the deceleration point for each vehicle. The script assumes the following breakup on the SCL's length:

- $l_{1}$ : A distance from the gore where all drivers are decelerating.

- $l_{2}$ : A distance upstream $l_{1}$ where drivers are likely decelerating at their selected deceleration rate.

- $l_{3}$ : A distance upstream $l_{2}$ where drivers may have started deceleration or are maintaining a constant speed.

Based on this assumption, the script does the following:

- The script first calculates the deceleration rate from each point in the speed profile to the gore and uses the points within the range of likely deceleration distance $l_{2}$ to establish a confidence interval for the deceleration rate.

- The first point of the speed profile, which would be located in $l_{2}$ or $l_{3}$, is checked against this confidence interval.

- If the deceleration rate from that point to gore is out of the confidence interval, the driver is assumed not to have started decelerating. The script 
then proceeds to the following points to find the first point that falls within the confidence interval.

- The first point within the confidence interval is defined as the deceleration point where the driver starts to decelerate at a constant rate.

- Subsequently, the script determines the initial speed at the deceleration point $\left(v_{i}\right)$, travel time on SCL from the diverge to deceleration point, and adjusted deceleration distance $(D)$ which is the distance travelled during deceleration from the deceleration point to gore.

- Using the speed at the gore as the final speed $\left(v_{\mathrm{f}}\right)$, the overall deceleration rate $(d)$ is calculated using the average of Equations 3.5 and 3.6. For the purpose of this research, a positive deceleration rate indicates a decrease in speed, while a negative deceleration rate indicates an increase in speed.

$\boldsymbol{d}_{\text {Time }}=\frac{v_{i}-v_{f}}{T}$

(Equation 3.5)

$d_{D i s}=\frac{v_{i}{ }^{2}-v_{f}{ }^{2}}{2 * D}$

(Equation 3.6)

It was observed that drivers do not use all of the available SCL length for deceleration. Drivers may require time to perceive the exit terminal and take the decision to diverge into the SCL, which may cause drivers to diverge a distance after the beginning of the SCL. Also, not using the full SCL length is related to drivers' behavior and choice of a diverge point. It was indicated in field data of the NCHRP 730 report that drivers do not use the full length of the SCL (Torbic, et al., 2012). Therefore, there was a need to calculate a variable that would explain how much of the available SCL length is used for deceleration. By knowing the actual length of the deceleration lane $(L)$, the 
utilization rate $(U)$ was calculated as $D / L$. Figures $3.5,3.6,3.7$, and 3.8 describe the script's methodology.

The script was run for all lanes using 95\% confidence interval. As the shortest SCL (Island Park Drive E-N) has a length of $58 \mathrm{~m}, l_{l}$ was taken $50 \mathrm{~m}$. It was found that many vehicles start deceleration inside the first $150 \mathrm{~m}$ before gore, which means that $l_{2}$ is less than $100 \mathrm{~m}$. Thus, $l_{2}$ was assumed to be $50 \mathrm{~m}$. The script was run for a total number of 41,045 speed readings on all seven limited-length SCLs. Figure 3.9 and 3.10 summarize the speed profiles beforehand after-processing; the script output includes:

- New overall deceleration rates calculated from the new deceleration point to the gore.

- Diverge speed at the diverge point onto the SCL.

- Initial speed at which a vehicle starts deceleration.

- Final/gore speed.

- Total travelled distance on the SCL.

- Deceleration distance used by the driver in deceleration mode.

- Time to decelerate, which is the time spent from diverge to deceleration points.

- Distance to decelerate, which is the distance spent from diverge to deceleration point.

Finally, utilization rate was calculated by dividing the deceleration distance by available SCL length; Figure 3.10 is a schematic of the estimated parameters after reprocessing. 


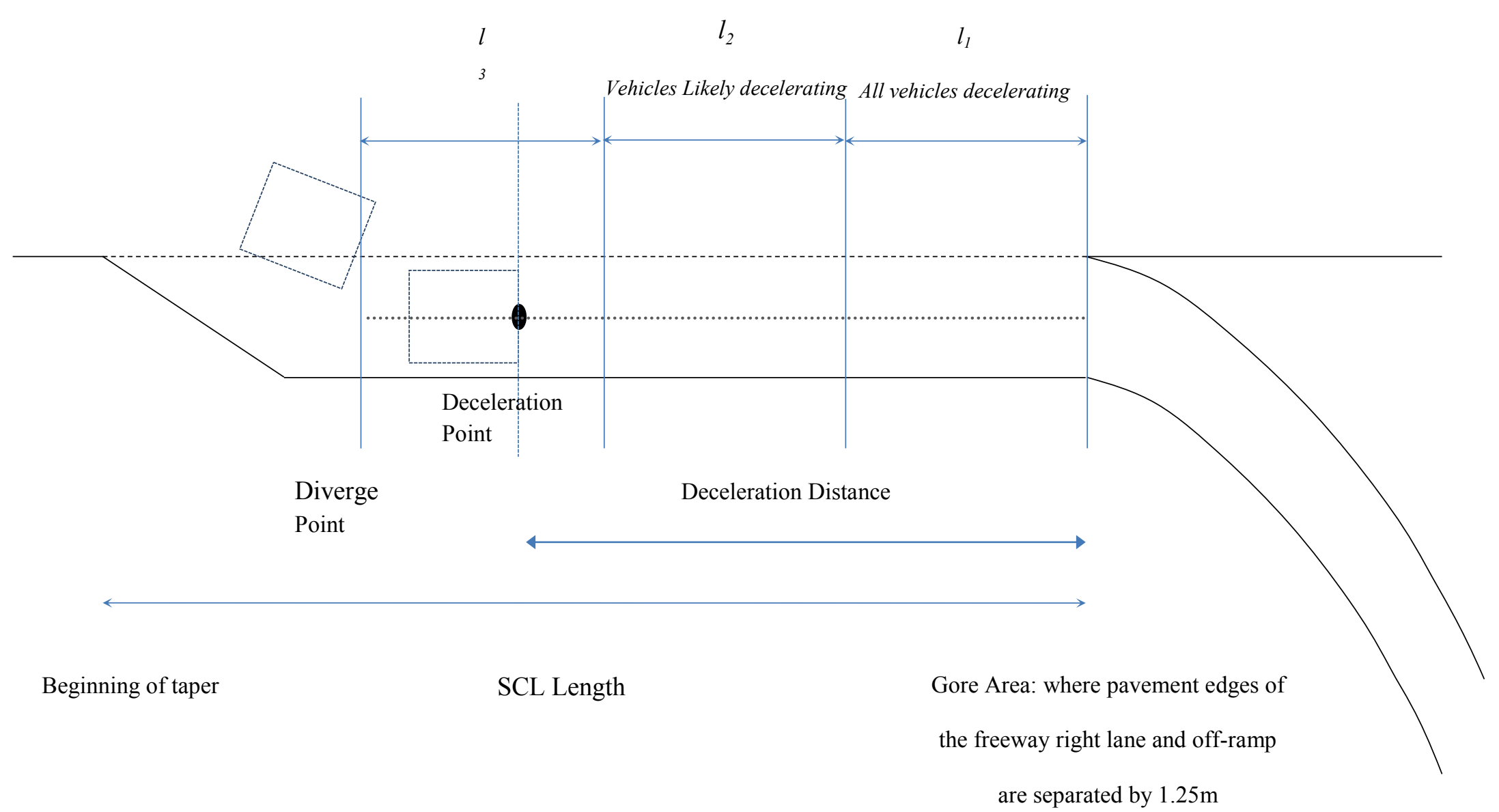

Figure 3.5: Schematic of SCL Lengths. 


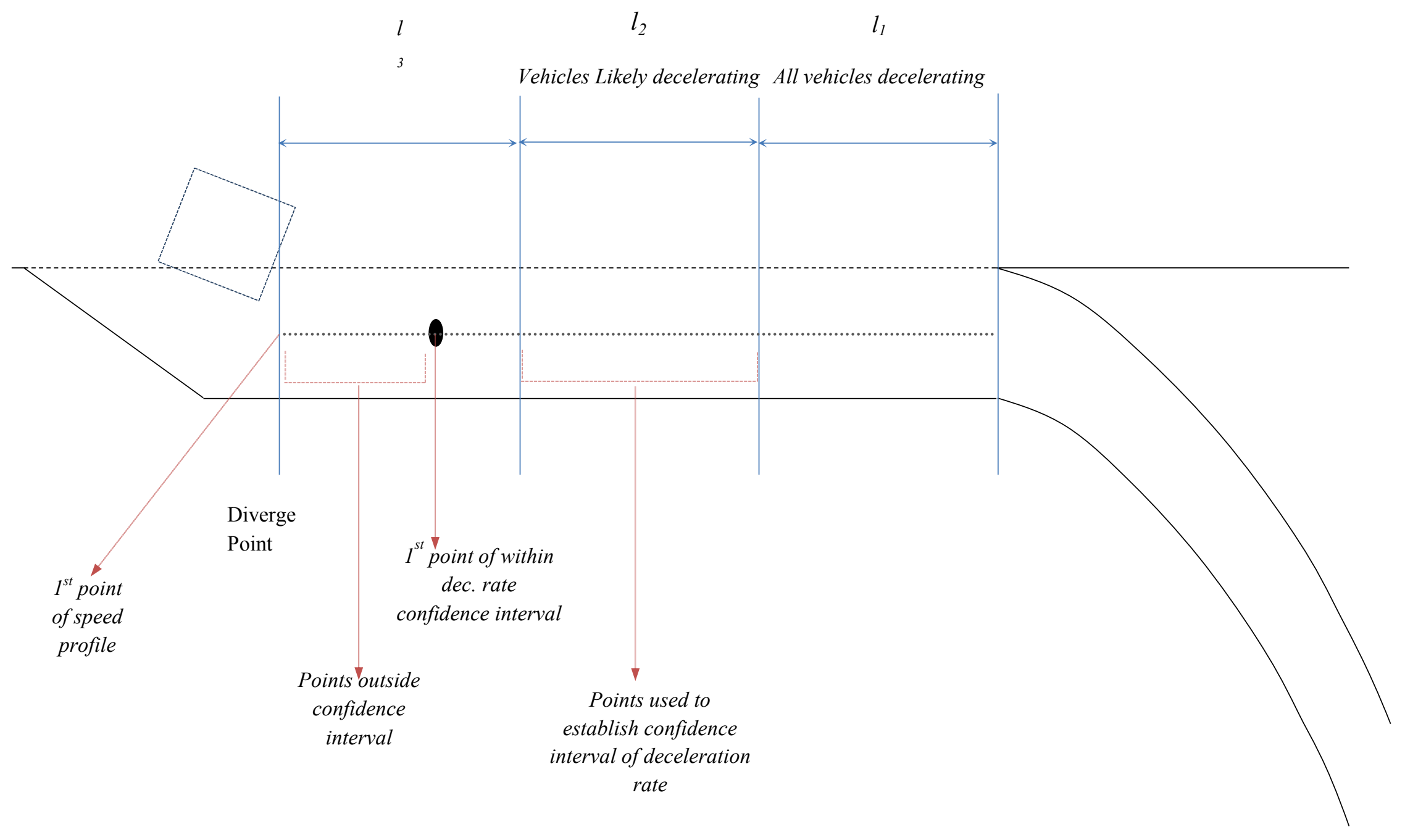

Figure 3.6: Long SCL and Deceleration Point within $\mathrm{I}_{3}$. 
Vehicles Likely decelerating All vehicles decelerating

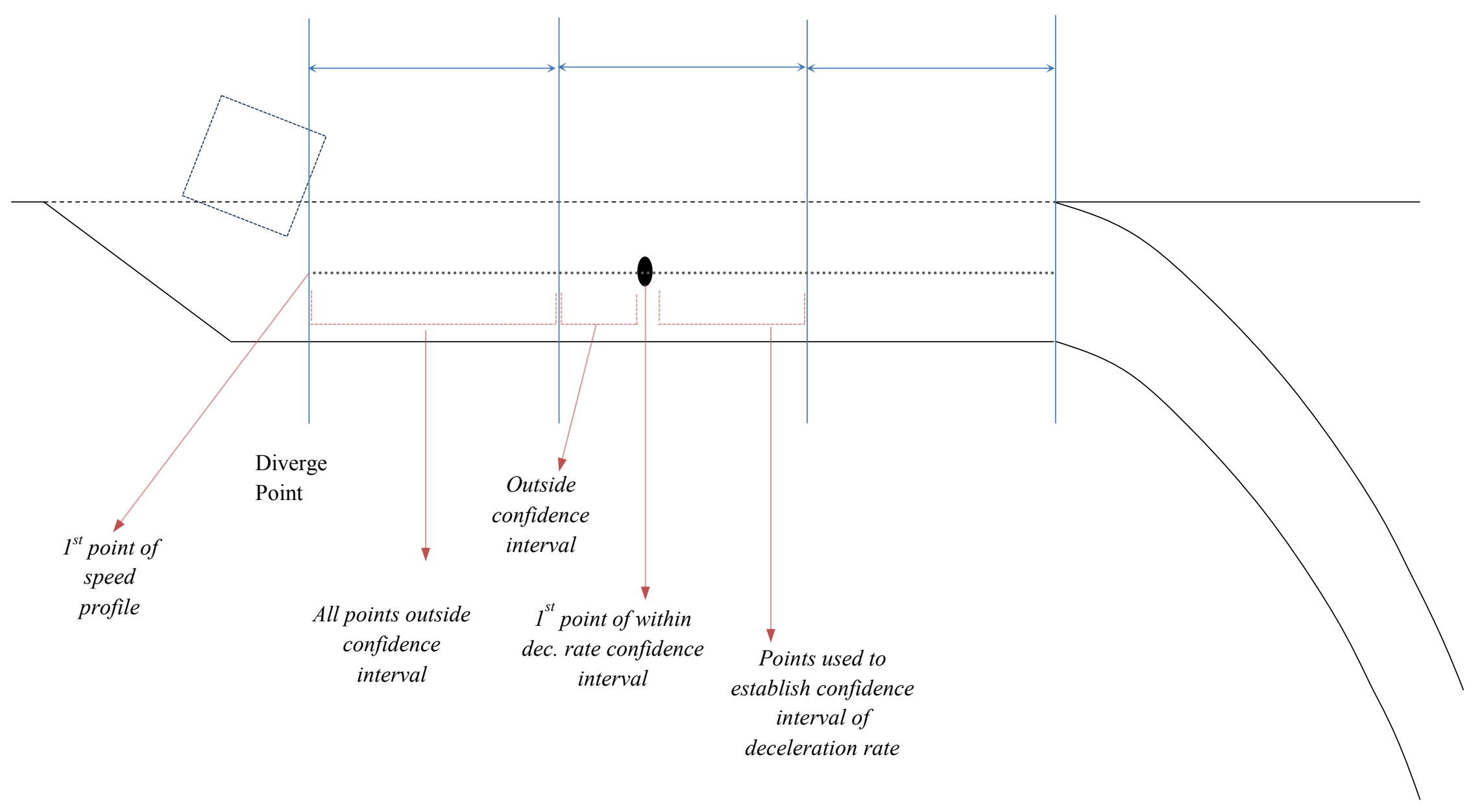

Figure 3.7: Long SCL and Deceleration Point within $\mathrm{I}_{2}$. 
$\leq l_{2}$

Vehicles Likely decelerating

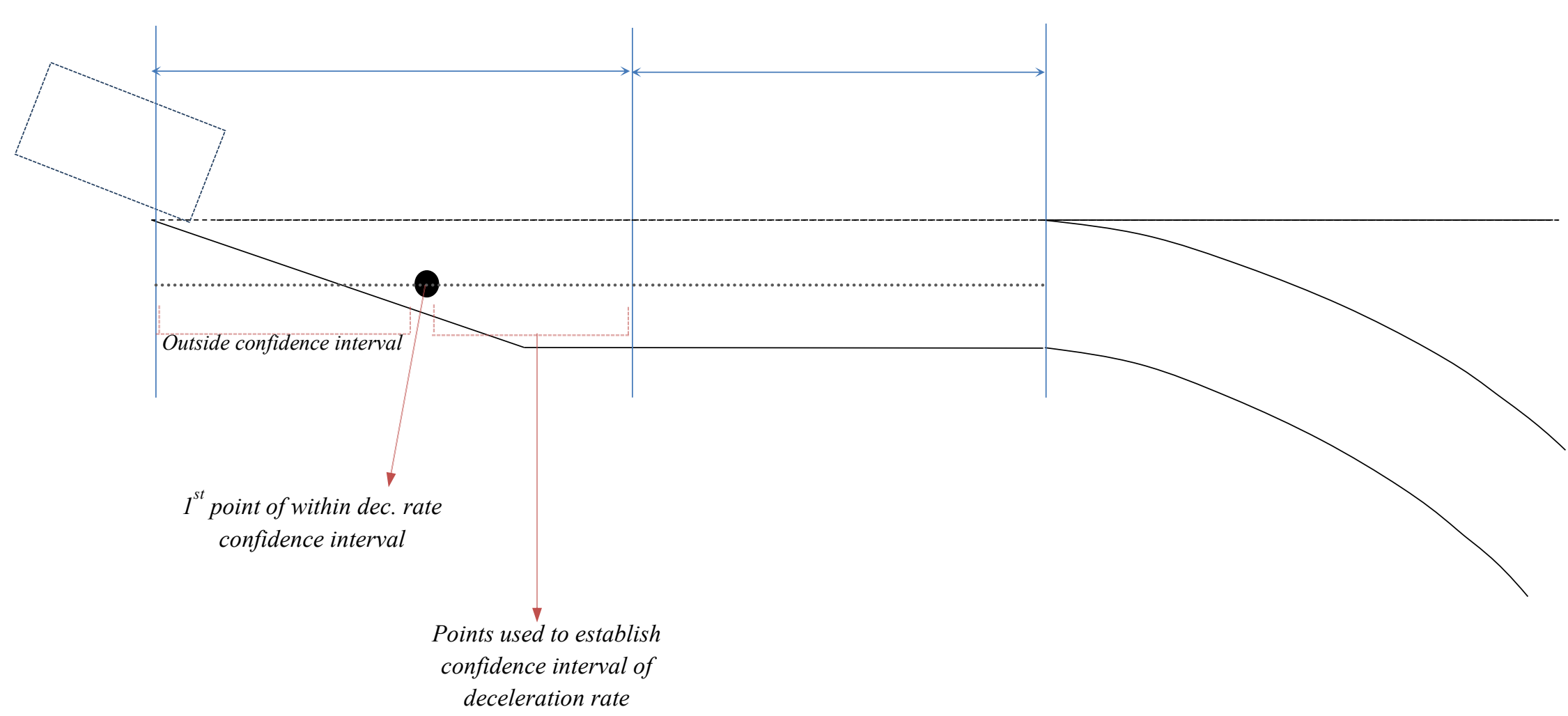

Figure 3.8: Short SCL and Deceleration Point within $\mathrm{I}_{2}$. 


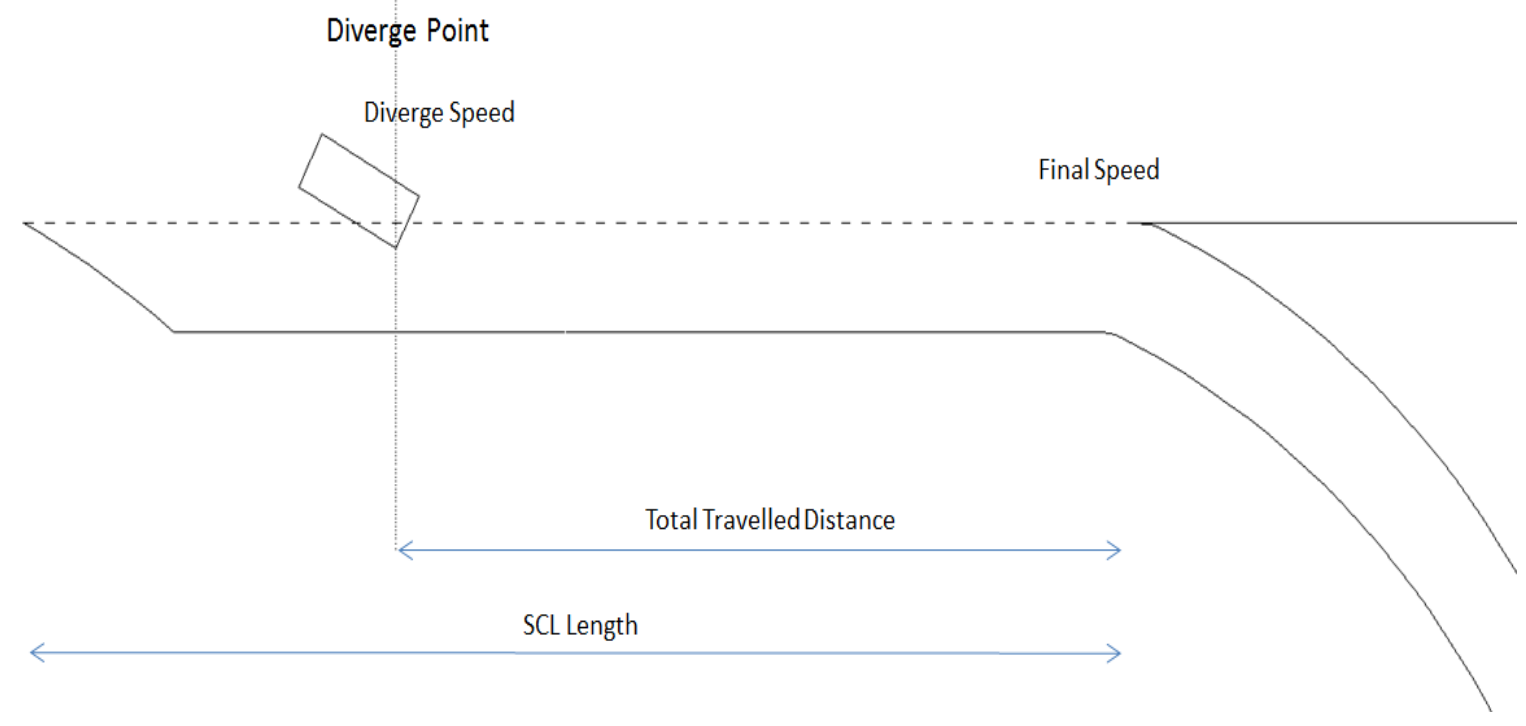

Figure 3.9: Speed Profiles before Reprocessing

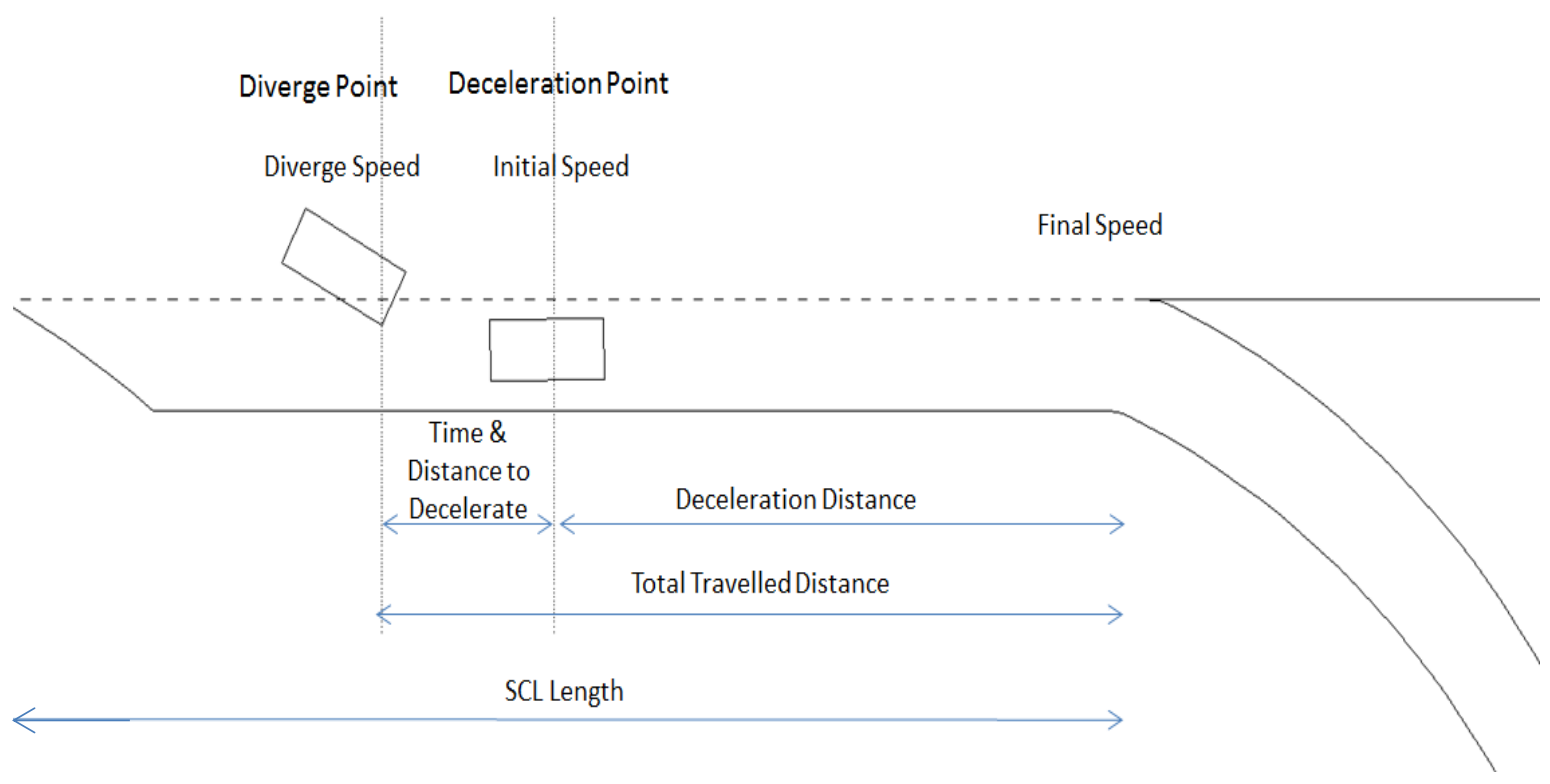

Figure 3.10: Final Speed Profiles after Reprocessing 


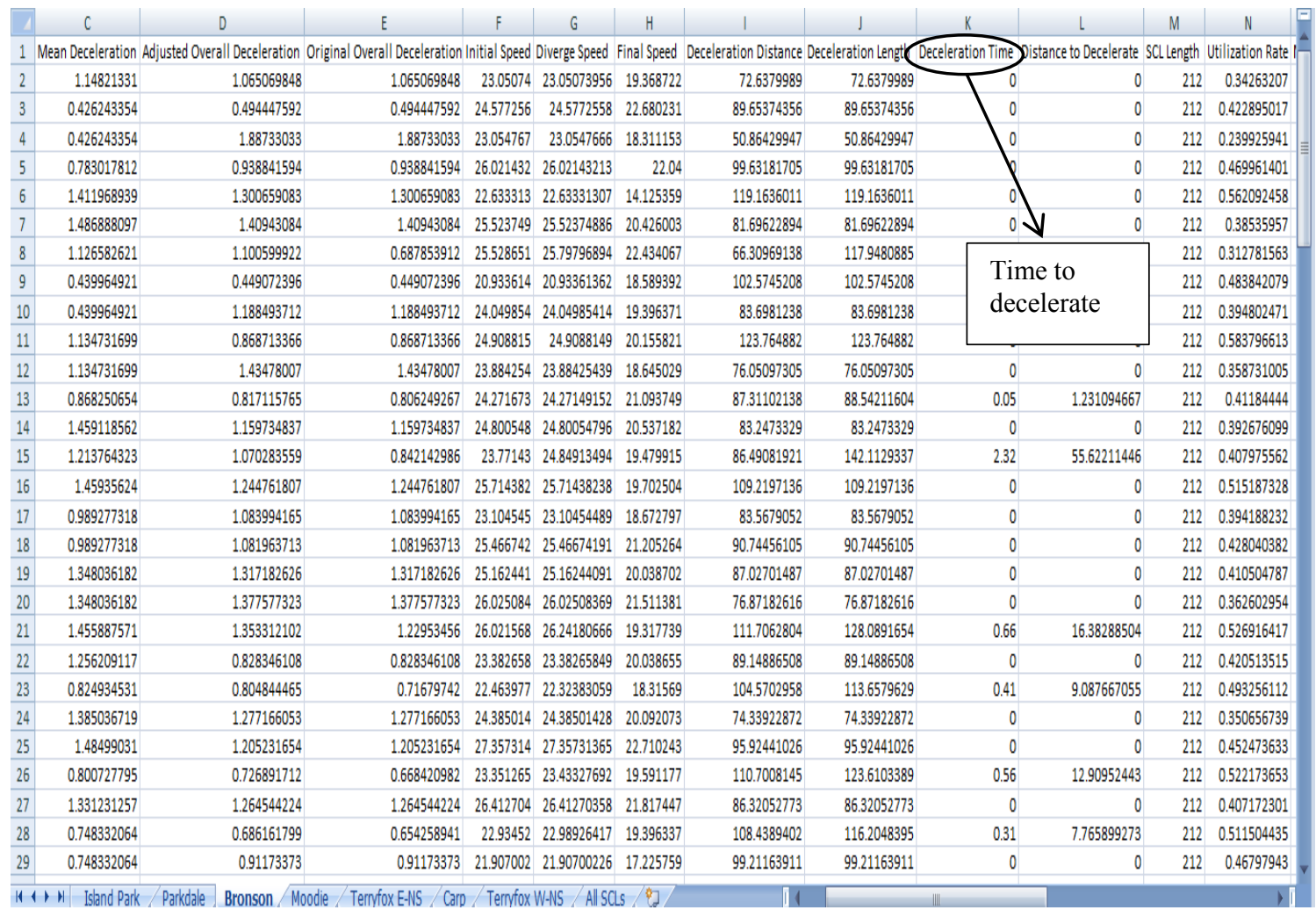

\section{Figure 3.11: Summary of Re-processed Data}

Figure 3.11shows a sample excel sheet for Bronson Ave (W-NS) after reprocessing.

For the purpose of this study, any profiles with negative overall decelerations (increase in peed) were removed as they violate the assumption of deceleration on the SCL. Trucks were not analyzed separately from passenger cars as they do not to have any significant effect on the driver behaviour on freeway diverge areas (El-Basha, 2006). The final database (Appendix 3) after reprocessing included 638 speed profiles on limited-length SCLs, while FRLs contained 866. Table 3.2 summarizes the numbers of available speed profiles by site. 
Table 3.2: Available Vehicle Speed Profiles at Every Site.

\begin{tabular}{|l|l|c|c|c|}
\hline No. & SCL Site & Length (m) & $\begin{array}{c}\text { Number of Tracked } \\
\text { SCL Vehicles }\end{array}$ & $\begin{array}{c}\text { Number of } \\
\text { Tracked FRL } \\
\text { Vehicles }\end{array}$ \\
\hline 1 & Island Park Drive (E-N) & 58 & 85 & 154 \\
\hline 2 & Parkdale Avenue (W-NS) & 179 & 90 & 128 \\
\hline 3 & Bronson Avenue (W-NS) & 212 & 90 & 119 \\
\hline 4 & Moodie Drive (W-NS) & 292 & 85 & 109 \\
\hline 5 & Terry Fox Drive (E-NS) & 388 & 114 & 124 \\
\hline 6 & Carp Road (E-NS) & 430 & 98 & 125 \\
\hline 7 & Terry Fox Drive(W-NS) & 446 & 76 & 866 \\
\hline \multicolumn{2}{|l|}{ Total Number of Speed Profiles } & 638 & 107 \\
\hline
\end{tabular}

SCL length includes the taper and is measured to the gore area where pavement edges of the freeway right lane and off-ramp are separated by $1.25 \mathrm{~m}$.

\subsection{Collision Data}

Collision data from the city of Ottawa were collected by Sarhan (2004) who studied developing explicit safety performance models on freeway merge and diverge areas. Data that are relevant only to the study sites were used. Total collision frequency was used for the purpose of exploring the premise of the followed approach of estimating the operational performance at the study sites. El-Basha (2006) tried to use the same data to model the effect of freeway geometry, traffic exposure, and speed data on collision prediction on freeway diverge areas. However, it was not possible to explicitly model safety due to the limited number of sites. The collision frequencies used were:

- The collision frequency for through and the SCL segments combined $\left(\mathrm{Col}_{\mathrm{ThDec}}\right)$.

- And the collision frequency for the SCL segments only $\left(\mathrm{Col}_{\mathrm{Dec}}\right)$.

Table 3.3 shows a summary of the collision data used. 
Table 3.3: Summary of Collision Data for the five years period by Site

\begin{tabular}{|l|r|c|c|}
\cline { 3 - 4 } \multicolumn{2}{c|}{} & \multicolumn{2}{c|}{ Collisions } \\
\hline \multirow{2}{*}{ SCL } & SCL Length & $\begin{array}{c}\text { Through and } \\
\text { SCL }\left(\text { Col }_{\text {ThDec }}\right)\end{array}$ & SCL(Col \\
\hline Island Park Drive E-NS & 58 & 76 & 8 \\
\hline Parkdale Avenue W-NS & 179 & 110 & 17 \\
\hline Bronson Avneue W-NS & 212 & 60 & 16 \\
\hline Moodie Drive W-NS & 292 & 72 & 5 \\
\hline Terry Fox Drive E-NS & 388 & 15 & 3 \\
\hline Carp Road E-NS & 430 & 21 & 3 \\
\hline Terry Fox Drive W-NS & 446 & 18 & \\
\hline
\end{tabular}




\section{Chapter Four}

\subsection{Data Analysis}

A detailed descriptive summary of the data used is covered in this Chapter. Statistical features of the data before and after reprocessing are presented and discussed. The first section of this chapter will present the statistical distributions of the main parameters defining the final reprocessed speed profiles, which are speeds (initial and final), deceleration rate, and utilization rate. Then, summary of the normal distribution testing for the data is followed. After that, two sets of statistical testing of variance (ANOVA) single factor mean of SCLs speed parameters are covered to check for homogeneity of the data samples. Finally, summary of the differences between the original data as processed by El-Basha's (2006) and the reprocessed data in this research on the SCLs will follow.

\subsection{Summary of Reprocessed Data}

New values for the mean and standard deviation of every parameter were obtained after applying the Matlab script. Table 4.1 shows the mean and standard deviation values for each parameter and the correlation coefficients of these parameters on each site and for all sites combined. The detailed steps and specific assumptions in applying the presented methodologies to the study SCLs are presented in Chapter 5.0. 
Table 4.1: Distribution of Input Parameters on Study Sites.

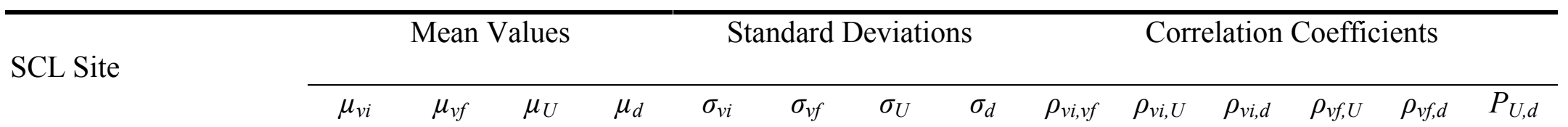

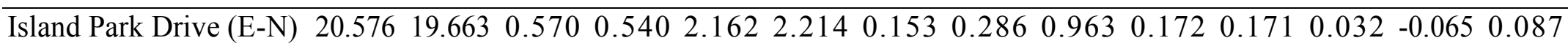

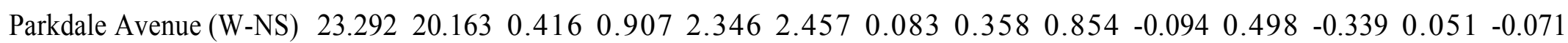

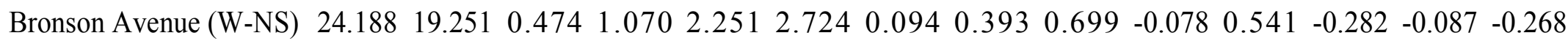

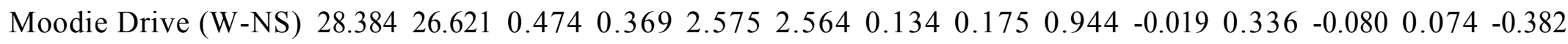

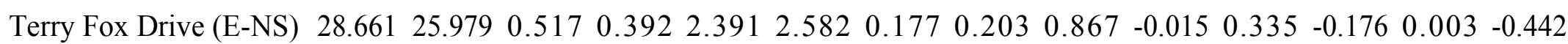

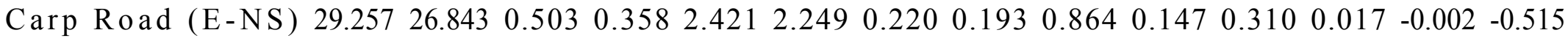

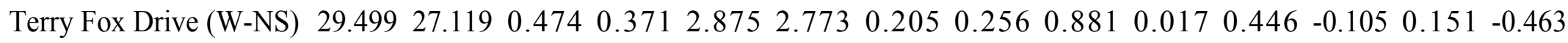

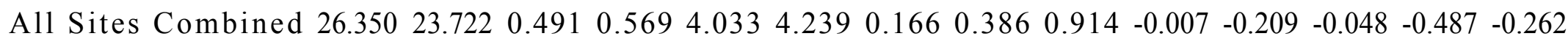

Speeds are in $\mathrm{m} / \mathrm{s}$; deceleration rate is in $\mathrm{m} / \mathrm{s}^{2}$; and utilization rate is unitless.

Where,

- $\quad v_{i}$ is initial speed, $v_{f} i s$ final speed, $U$ is utilization rate, and $d$ is deceleration rate.

- $\quad \mu$ is mean value, $\sigma$ is standard deviation value, and $\rho$ is the correlation coefficient. 


\subsection{Statistical Tests on Reprocessed Data}

To apply the proposed probabilistic approaches discussed in Chapter 5.0, statistical properties of the data should be studied. All statistical testing was performed at a level of significance of 5\%, which is frequently used in highway research. It should be noted that the distributions of the parameters or any statistical differences within the data sample are related to the drivers' behavior and choices of initial and final speeds, deceleration, and utilization rates.

\subsubsection{Distribution Testing of SCL Speed Profiles}

Using Anderson Darling test (Bellosta, 2013), all parameters were tested. The null hypothesis is that the distribution is normal. If the $p$-value is less than the selected level of significance, the null hypothesis is rejected. Similarly, if the test statistic (AD value) is higher than the critical value, the null hypothesis is rejected. At a level of significance ( $p$ value) of $5 \%$, it was found that the normal distribution is applicable for all parameters except for the utilization rate at Carp Road E-NS (an AD value of 3.22 and a $p$-value of 0.021 ) and the deceleration rate at Island Park Drive E-N (an AD value of 2.778 and a $p$ value of 0.036 ). Since all data follow a normal distribution except only for these two cases (out of 28 cases), all data were considered to have a normal distribution. Table 4.2 shows a summary of the normality test done in R statistical language using the package "ADGofTest." 
Table 4.2: Distribution Testing Summary of Reprocessed Data

\begin{tabular}{|c|c|c|c|c|}
\hline $\mathrm{SCL}$ & Parameter & AD Test Value & $p$-Value & Normality Test Result \\
\hline \multirow{4}{*}{ Island Park Drive E-N } & $v_{i}$ & 0.310 & 0.930 & Normal \\
\hline & $v_{f}$ & 0.510 & 0.737 & Normal \\
\hline & $U$ & 0.427 & 0.822 & Normal \\
\hline & $d$ & 2.778 & 0.036 & Not Normal \\
\hline \multirow{4}{*}{ Parkdale Avenue W-NS } & $v_{i}$ & 0.744 & 0.523 & Normal \\
\hline & $v_{f}$ & 0.628 & 0.621 & Normal \\
\hline & $U$ & 0.613 & 0.636 & Normal \\
\hline & $d$ & 0.646 & 0.605 & Normal \\
\hline \multirow{4}{*}{ Bronson Avenue W-NS } & $v_{i}$ & 0.213 & 0.987 & Normal \\
\hline & $v_{f}$ & 1.237 & 0.254 & Normal \\
\hline & $U$ & 0.534 & 0.712 & Normal \\
\hline & $d$ & 0.936 & 0.392 & Normal \\
\hline \multirow{4}{*}{ Moodie Drive W-NS } & $v_{i}$ & 0.303 & 0.936 & Normal \\
\hline & $v_{f}$ & 0.512 & 0.734 & Normal \\
\hline & $U$ & 0.281 & 0.951 & Normal \\
\hline & $d$ & 0.508 & 0.738 & Normal \\
\hline \multirow{5}{*}{ Terry Fox E-NS } & $v_{i}$ & 0.627 & 0.623 & Normal \\
\hline & $v_{f}$ & 0.303 & 0.936 & Normal \\
\hline & $U$ & 1.812 & 0.117 & Normal \\
\hline & $d$ & 1.472 & 0.183 & Normal \\
\hline & $v_{i}$ & 0.209 & 0.988 & Normal \\
\hline
\end{tabular}




\begin{tabular}{|c|c|c|c|c|}
\hline \multirow{3}{*}{ Carp Road E-NS } & $v_{f}$ & 0.211 & 0.987 & Normal \\
\hline & $U$ & 3.112 & 0.024 & Not Normal \\
\hline & $d$ & 0.806 & 0.476 & Normal \\
\hline \multirow{4}{*}{ Terry Fox W-NS } & $v_{i}$ & 0.882 & 0.425 & Normal \\
\hline & $v_{f}$ & 0.223 & 0.983 & Normal \\
\hline & $U$ & 2.199 & 0.072 & Normal \\
\hline & $d$ & 1.924 & 0.101 & Normal \\
\hline
\end{tabular}

\subsubsection{ANOVA Single Factor Mean Tests}

The reprocessed data were tested in this section to identify locations where diverging drivers decelerate on the FRL. This testing was performed as it was observed that mean initial and diverge speeds at SCLs and speeds at FRL are higher at longer SCL sites. Table 4.3 shows a summary of the mean FRL speed, diverge speed onto SCL, and initial speed on SCL. Difference between mean FRL speeds and mean Diverge speeds decrease at longer sites.

For the purpose of identifying locations where diverging drivers decelerate on the FRL, analysis of variance (ANOVA) test is used to check if there is a difference between speed parameters at different sites. Two sets of ANOVA tests were performed on the reprocessed data as explained below. 
Table 4.3: Summary of Mean FRL, Diverge, and Initial Speeds

\begin{tabular}{|c|c|c|c|c|c|}
\hline SCL Name & $\begin{array}{c}\text { Average } \\
\text { Running } \\
\text { Rpeeds } \\
(\mathrm{m} / \mathrm{s})\end{array}$ & $\begin{array}{c}\text { Mean } \\
\text { Diverge } \\
\text { Speed Data } \\
(\mathrm{m} / \mathrm{s})\end{array}$ & $\begin{array}{c}\text { Mean } \\
\text { Initial } \\
\text { Speed } \\
(\mathrm{m} / \mathrm{s})\end{array}$ & $\begin{array}{c}\text { Difference } \\
\text { Between Average } \\
\text { FRL \& Mean } \\
\text { Diverge Speeds } \\
(\mathrm{m} / \mathrm{s})\end{array}$ & $\begin{array}{c}\text { Difference } \\
\text { Between Mean } \\
\text { Diverge and } \\
\text { Initial Speeds } \\
(\mathrm{m} / \mathrm{s})\end{array}$ \\
\hline $\begin{array}{c}\text { Island Park } \\
\text { Drive (E-N) }\end{array}$ & 24.675 & 20.576 & 20.576 & 4.099 & 0.000 \\
\hline $\begin{array}{c}\text { Parkdale Ave. } \\
\text { (W-NS) }\end{array}$ & 26.850 & 23.277 & 23.292 & 3.573 & -0.015 \\
\hline $\begin{array}{c}\text { Bronson Ave. } \\
\text { (W-NS) }\end{array}$ & 26.842 & 24.254 & 24.188 & 2.588 & 0.065 \\
\hline $\begin{array}{c}\text { Moodie Drive } \\
\text { (W-NS) }\end{array}$ & 28.947 & 28.356 & 28.384 & 0.591 & -0.028 \\
\hline $\begin{array}{c}\text { Terry Fox } \\
\text { Drive (E-NS) }\end{array}$ & 29.114 & 28.965 & 28.661 & 0.149 & 0.304 \\
\hline $\begin{array}{c}\text { Carp Road (E- } \\
\text { NS) }\end{array}$ & 29.864 & 29.586 & 29.257 & 0.278 & 0.179 \\
\hline $\begin{array}{c}\text { Terry Fox } \\
\text { Drive (W-NS) }\end{array}$ & 29.850 & 29.678 & 29.499 & 0.172 & 0.330 \\
\hline
\end{tabular}

\section{A. Initial Speeds at Different SCL Sites}

The purpose of this set of ANOVA tests is to identify long and short SCLs. Long SCLs are expected to have higher initial speeds as drivers would have enough distance to decelerate on the SCL. On the other hand, short SCLs are expected to have lower initial speeds as drivers would not have enough deceleration distance on the SCL. Therefore, drivers may start deceleration on the FRL. The null hypothesis in ANOVA test is that the means of initial speed at all long SCL sites are equal. If the $p$-value is less than the selected level of significance or if the calculated test statistic " $F_{\text {calc." }}$ " is greater than the critical value " $F_{\text {crit }}$ ", the null hypothesis is rejected.

First, initial speeds at all sites were tested at 5\% level of significance. If the null hypothesis was rejected, the data would not be homogenous and initial speeds would 
correspond to both long and short SCLs. Then, the shortest site was dropped and the test was repeated for the remaining sites. This process was followed until, for all remaining sites, mean initial speeds were found to have no statistically significant difference. The results of all ANOVA tests are available in Appendices 4. As shown in the appendix, no significant difference was found between the mean initial speeds at Terry Fox Drive (ENS), Carp Road (E-NS), and Terry Fox Drive (W-NS) $\left(p\right.$-value $=0.061, F_{\text {calc. }}=2.829$, and $\left.F_{\text {crit }}=3.027\right)$. Therefore, these sites were considered as long SCLs, while the rest of the SCLs were considered to be short. This information will be helpful in applying the methodology of Monte Carlo Simulation (MCS) discussed in Section 5.4. The results of this test imply that deceleration on adjacent FRL could be happening on short SCLs. This conclusion is drawn since all vehicles are travelling on the same freeway and should have the same speed distribution.

\section{B. FRL and Diverge Speeds at Each Site}

ANOVA test was performed at each site to test the significance of the difference between diverge speeds and FRL speeds at each site. The null hypothesis is that the means of diverge speeds and initial speeds at each site are equal. If the $p$-value is less than the level of significance or if the test statistic " $F_{\text {calc. }}$ " is greater than the critical value " $F_{\text {crit }}$ ", the null hypothesis is rejected. At a 5\% level of significance, it was found that diverge and FRL speeds are homogenous at Moodie Drive W-NS, Terry Fox Drive (ENS), Carp Road (E-NS), and Terry Fox Drive (W-NS) (Appendix 5). This test indicates homogeneity between diverge and FRL speeds at each site and does not necessarily imply whether deceleration takes place on the SCLs or not. FRL speeds are affected by 
the drivers' behaviour on the SCL. In case drivers diverge at low speeds onto the SCL, if it was judged to be short, the following vehicles on the FRL would be affected and would travel at low speeds. Furthermore, some vehicles may travel on the FRL at low speeds as slow traffic is expected to keep to the right lane of the road. Therefore the test cannot be used to identify if deceleration happens on the FRL before merging onto the SCL. But only to test the homogeneity of SCL diverge speeds and FRL speeds. Summary of the final test for four homogeneous sites is in Table 4.4.

Table 4.4: Summary of ANOVA at Homogeneous sites

\begin{tabular}{|l|l|l|l|}
\hline SCL & $p$-value & $F_{\text {calc. }}$ & $F_{\text {crit }}$ \\
\hline Moodie Drive W-NS & 0.088 & 2.935 & 3.890 \\
\hline Terry Fox Drive (E-NS) & 0.639 & 0.221 & 3.881 \\
\hline Carp Road (E-NS) & 0.395 & 0.726 & 3.884 \\
\hline Terry Fox Drive (W-NS) & 0.664 & 0.189 & 3.893 \\
\hline
\end{tabular}

\subsection{Comparison of Original and Reprocessed Data}

As mentioned in Section 3.4, a Matlab script was developed and applied in order to estimate the point where drivers start decelerating. In the original data by El-Basha (2006), the assumption was that drivers used the total travelled distance on the SCL for deceleration. But, if a driver enters a long SCL, they may maintain their speed for some time or even slightly accelerate before applying the brakes to decelerate. After applying the script (Appendix 2), new overall deceleration rates, deceleration distances, and initial 
speeds are estimated. Final speeds are to remain the same as they are the end conditions and are not be affected by the script.

\subsubsection{Time and Distance to Decelerate}

Time and distance between the diverge point to the deceleration point vary depending on the driver's choice of initial speed, available SCL length, utilization rate, and end condition of the SCL. Long SCLs are expected to have a longer time and distance to decelerate. Figures 4.1 and 4.2 show the vehicles time and distance to decelerate, respectively.

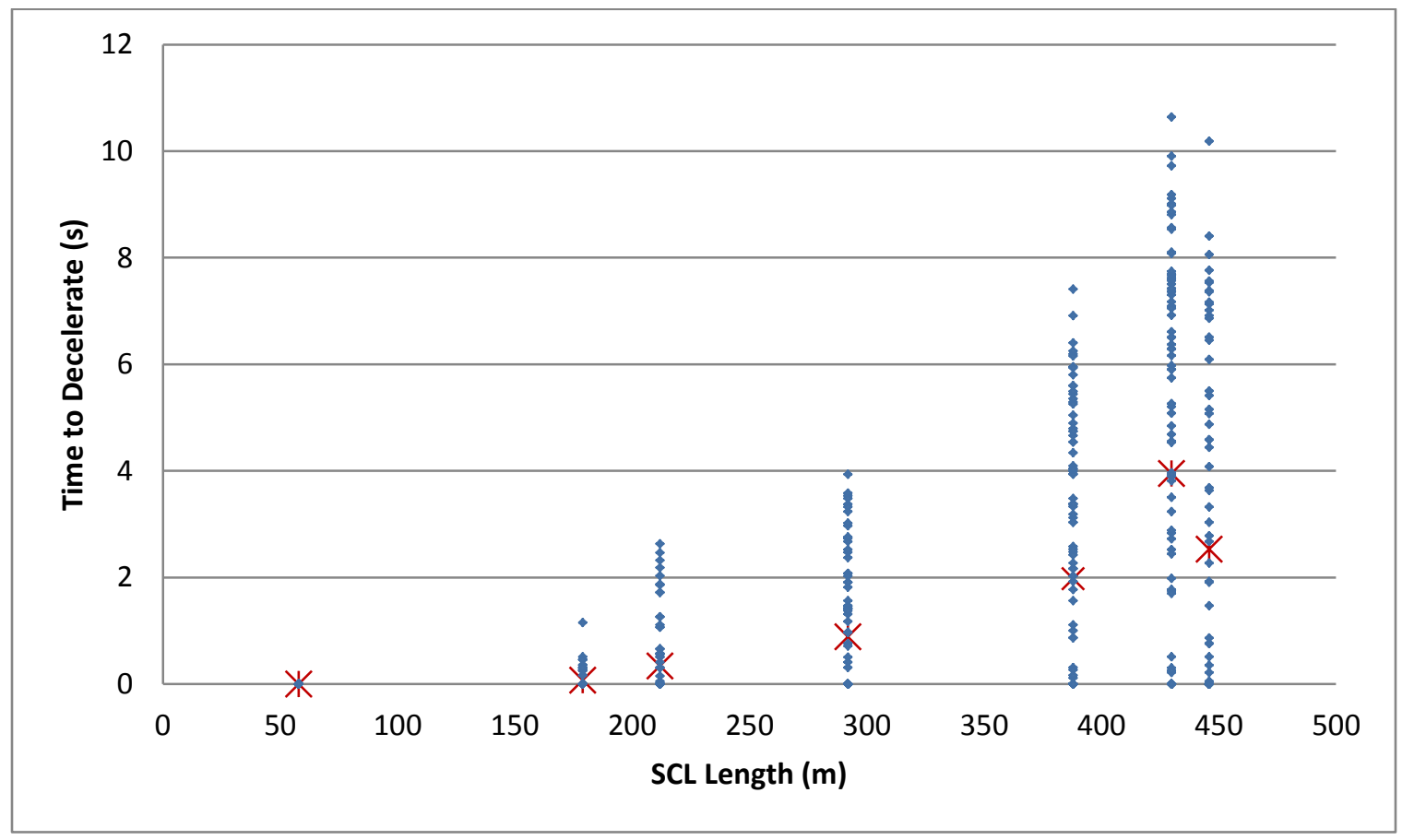

Figure 4.1: Time Spent on the SCL before Decelerating (dots represent each vehicle and $X$ represents the mean value of vehicles at each site). 


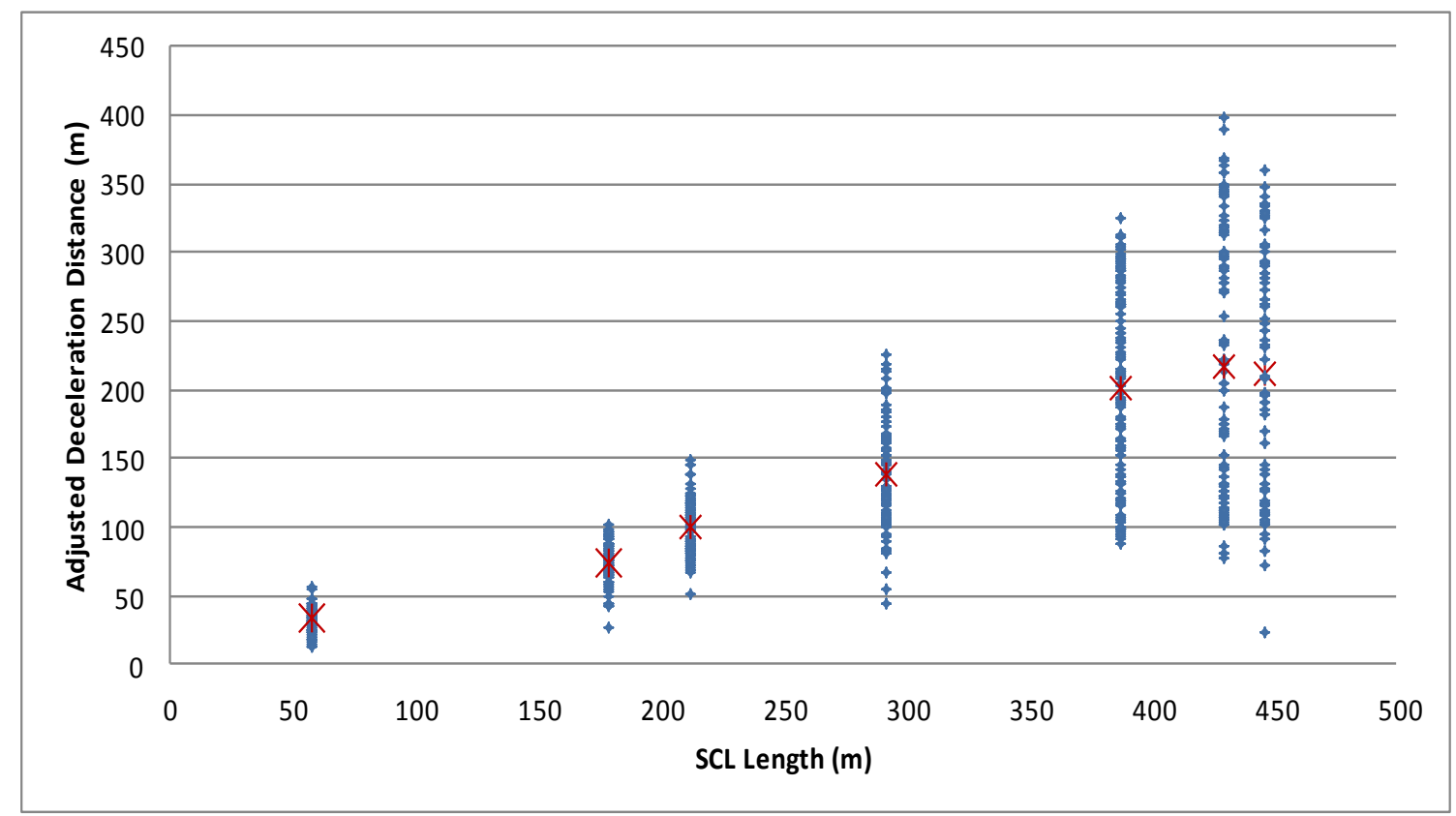

Figure 4.2: Distance Spent on the SCL before Decelerating (dots represent each vehicle and $X$ represents the mean value of vehicles at each site).

As shown in Figures 4.1 and 4.2, the time and distance to decelerate in Island Park Drive (length of $58 \mathrm{~m}$ ) are both zero as it is a very short SCL. Both values increase with the increase of the available SCL length. Terry Fox Drive W-NS has less mean distance and time to decelerate values than Carp Road E-NS, which could be related to the higher ramp's width and allowed speed at the ramp on Carp Road E-NS compared to Terry Fox Drive W-NS. The geometric characteristics could be found in (Appendix 1)

\subsubsection{Final Speeds}

As explained in Section 3.4, the script used for reprocessing was developed to estimate the deceleration point on SCLs. Therefore, new initial speeds were found at beginning of deceleration but final speed was not affected. Therefore, for all vehicles, there is no difference in the final speed before and after reprocessing the data. It was 
noticed that final speeds are high at SCLs with high ramp advisory speeds. In other words, the geometric configuration of the ramp affects the final speed at the SCL. Table 4.5 and Figure 4.3 show the relationship between each site's final speed and the ramp advisory speed. The table and figure show that as the ramps advisory speed increases (which indicates a more forgiving geometry), the drivers final speed increases.

Table 4.5: Mean Final Speed at Each Site and Relative Ramps Advisory Speeds

\begin{tabular}{|l|l|l|}
\hline SCL Site & Final Speed $(\mathrm{km} / \mathrm{h})$ & Ramp Advisory Speed $(\mathrm{km} / \mathrm{h})$ \\
\hline Island Park Drive (E-N) & 70.786 & 40 \\
\hline Parkdale Avenue (W-NS) & 72.587 & 50 \\
\hline Bronson Avenue (W-NS) & 69.303 & 40 \\
\hline Moodie Drive (W-NS) & 95.835 & 50 \\
\hline Terry Fox Drive (E-NS) & 93.523 & 50 \\
\hline Carp Road (E-NS) & 96.635 & 70 \\
\hline Terry Fox Drive (W-NS) & 97.627 & 60 \\
\hline
\end{tabular}

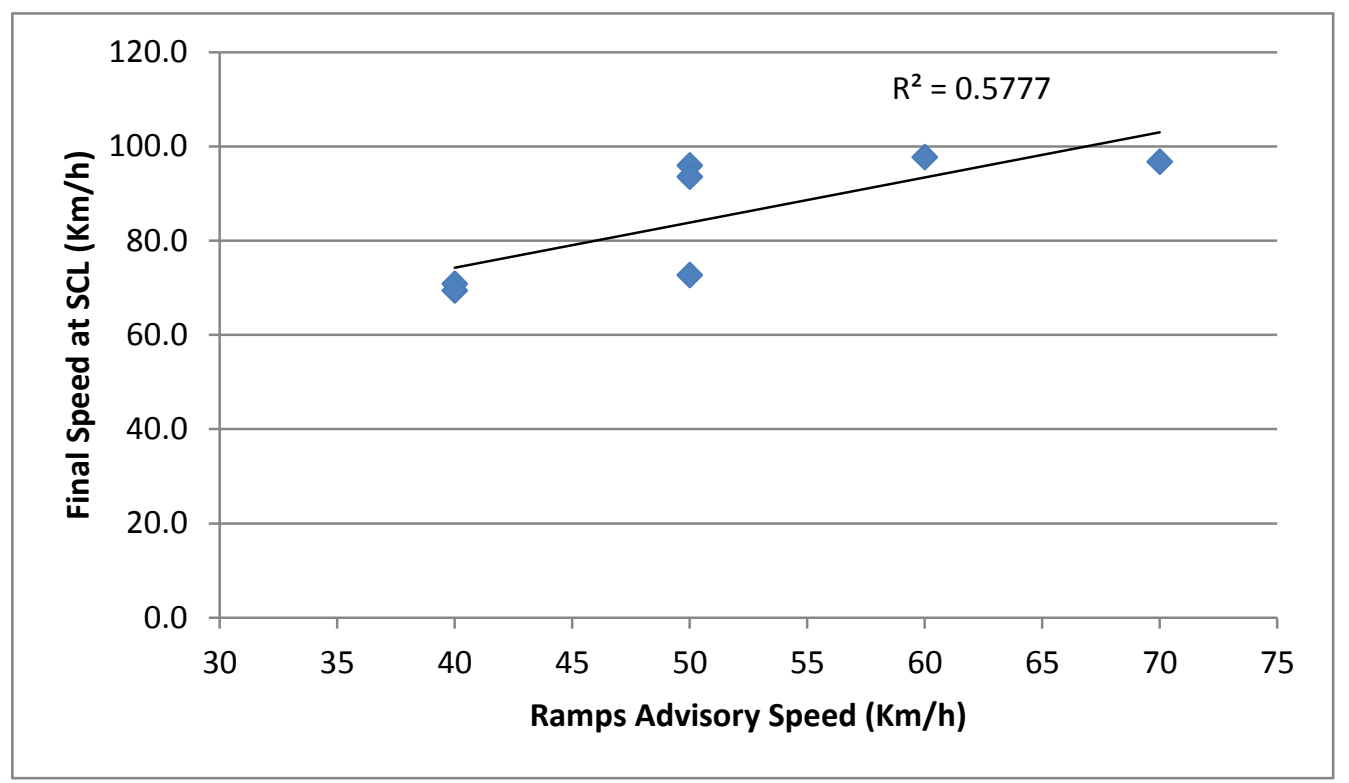

Figure 4.3: Ramp's Advisory Speeds and Mean Final Speeds at All Sites 


\section{Initial and Gore Speeds}

It was unknown whether drivers slightly accelerate, maintain their speeds, or slightly decelerate before reaching the point of effective deceleration. Thus, the initial speeds (at deceleration point), as estimated by the Matlab script, were compared to the diverge speeds (at diverge point) as shown in Figure 4.4.

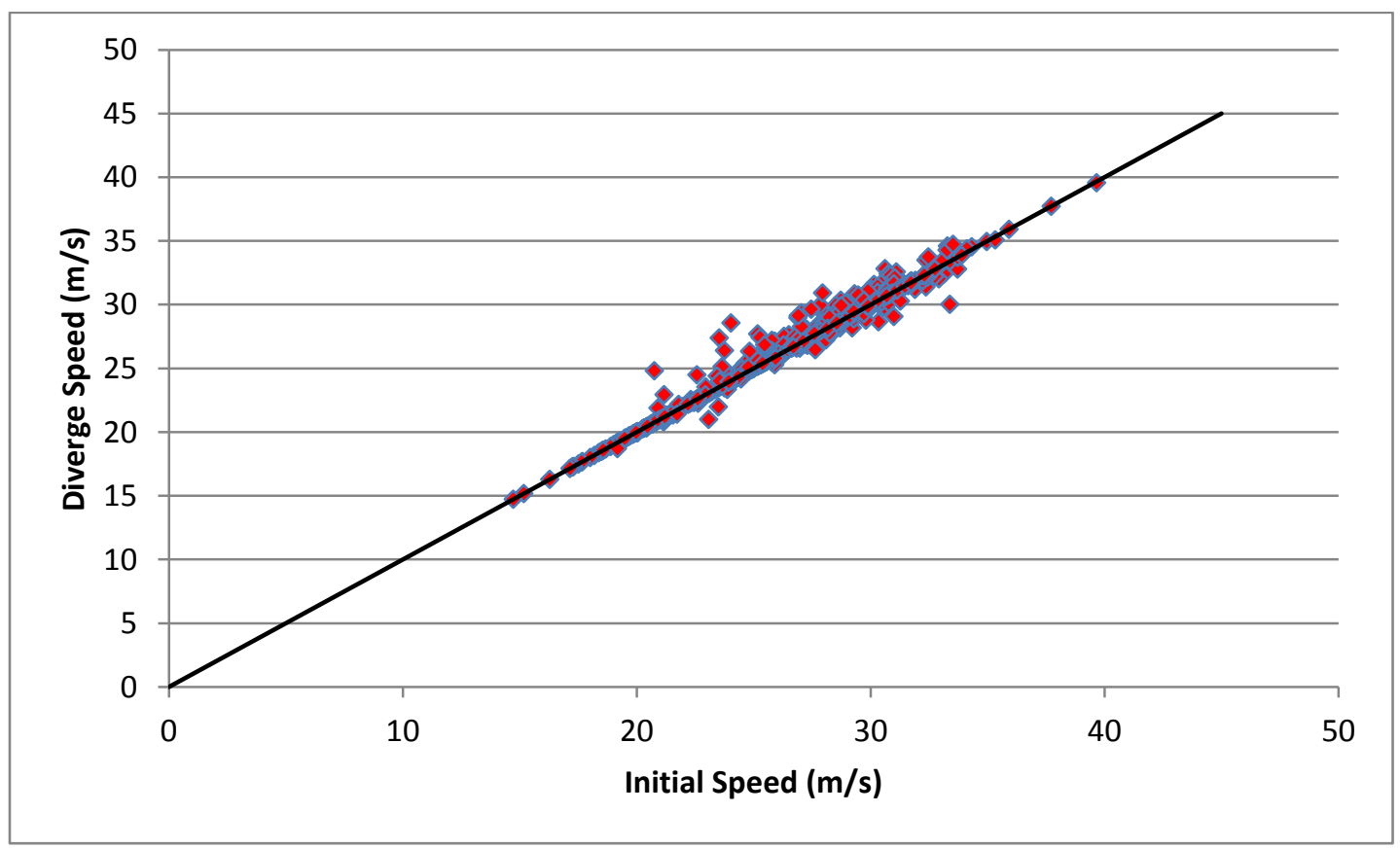

Figure 4.4: Comparison between Initial and Diverge Speeds

For all individual SCL vehicles, the difference between initial and diverge speeds ranged between -3.362 and $4.527 \mathrm{~m} / \mathrm{s}(-12.10$ and $16.30 \mathrm{~km} / \mathrm{h})$, which shows that some vehicles decelerated and some accelerated after diverging into the SCL. The average speed difference was $0.130 \mathrm{~m} / \mathrm{s}(0.47 \mathrm{~km} / \mathrm{h})$, which means that on average vehicles' speeds did not change considerably from the diverge to deceleration points. Using the line of equality, Figure 4 shows that the individual vehicle data are close to the line of 
equality between diverge and initial speeds. Therefore, the assumption that drivers start decelerating at the deceleration point is confirmed.

\subsubsection{Deceleration Distance and Total Travelled Distance}

After reprocessing the data, deceleration distances that start from actual deceleration point are estimated. The reduction from total travelled distance to deceleration distance is affected by the driver's choice of not decelerating at the diverge point. Figure 4.5 is a summary of the mean deceleration distance and total travelled distance on SCL at each site.

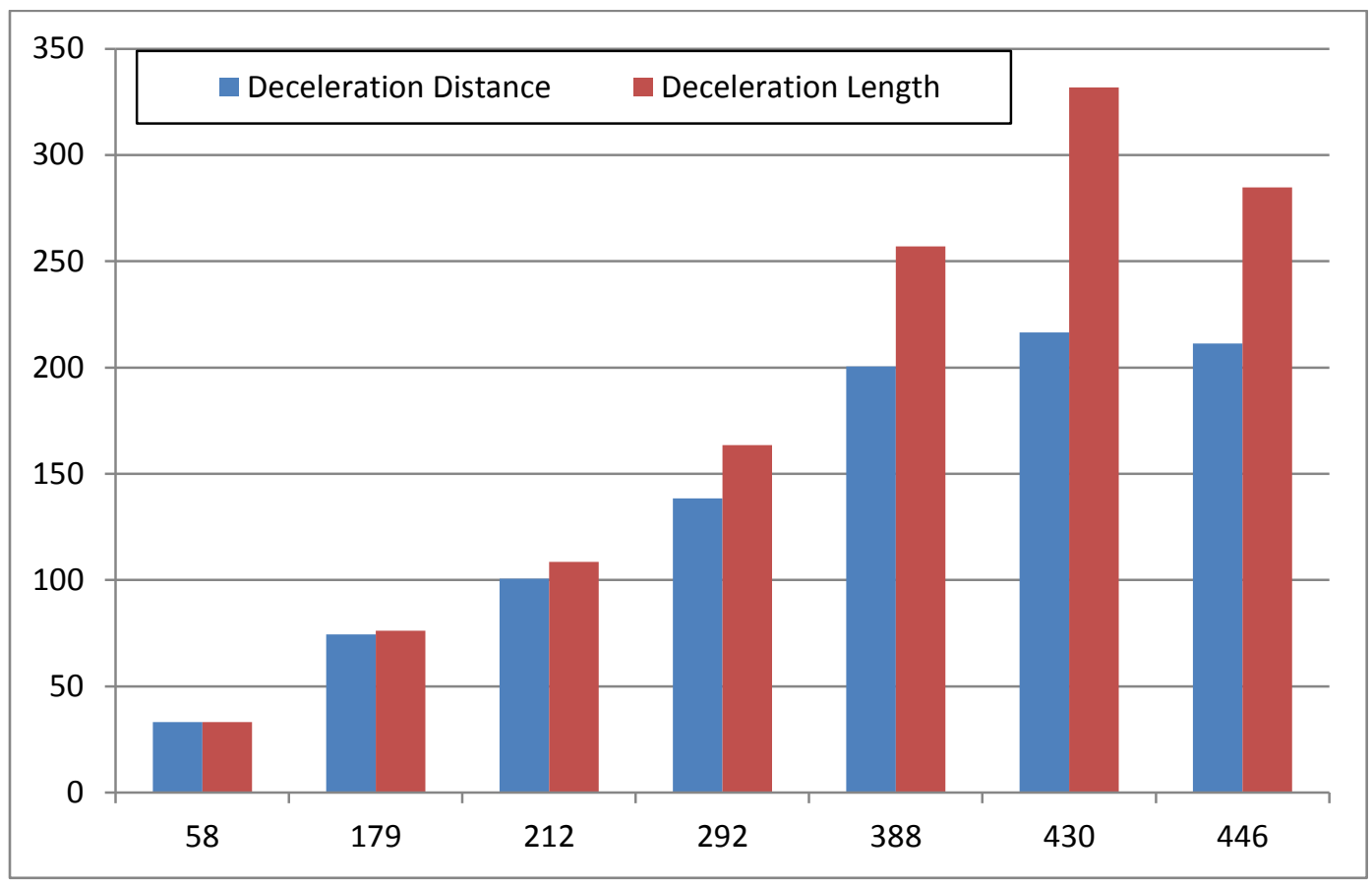

Figure 4.5: Deceleration Distance and Total Travelled Distance.

The difference between total travelled distance and deceleration distance follows the same trend of the time and time and distance to decelerate on every SCL. As time 
spent to decelerate increases, distance to decelerate will increase and available deceleration distance decreases. For all vehicles' profiles, the difference between total travelled distance and deceleration distance ranged between 0.00 at Island Park Drive E$\mathrm{N}$ to1 $15.39 \mathrm{~m}$ at Carp Road E-NS. Mean difference for all vehicle data is $40.04 \mathrm{~m}$ and the standard deviation is $43.58 \mathrm{~m}$, which indicates potentially misleading results if the total travelled distances were used without reprocessing.

\subsubsection{Overall Deceleration Rate}

After reprocessing the data, it was anticipated that original overall deceleration rate values (based on the total travelled distance and total time spent on the SCL) would increase after using the estimated deceleration distance and time in the calculation process. As mentioned in Section 3.3, the overall deceleration calculation depends on the average of both distance deceleration and time deceleration, Equations 3.3 and 3.4. If the total deceleration distance and time decrease, the overall deceleration would increase. Figures 4.6 and 4.7 show the difference between the mean and standard deviations of the overall deceleration rates before and after reprocessing the data. 


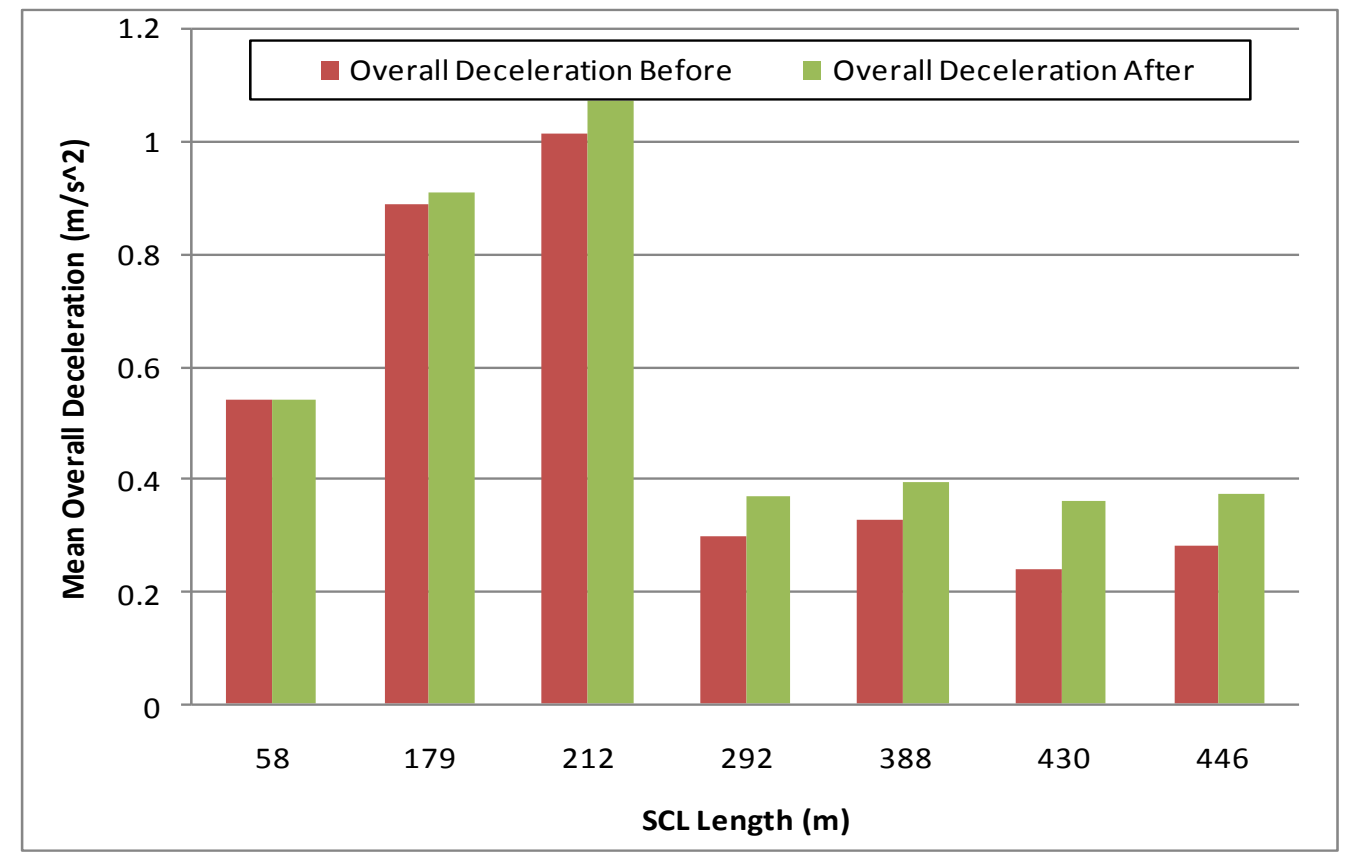

Figure 4.6: Mean Overall Deceleration Rate before and after Reprocessing the Data.

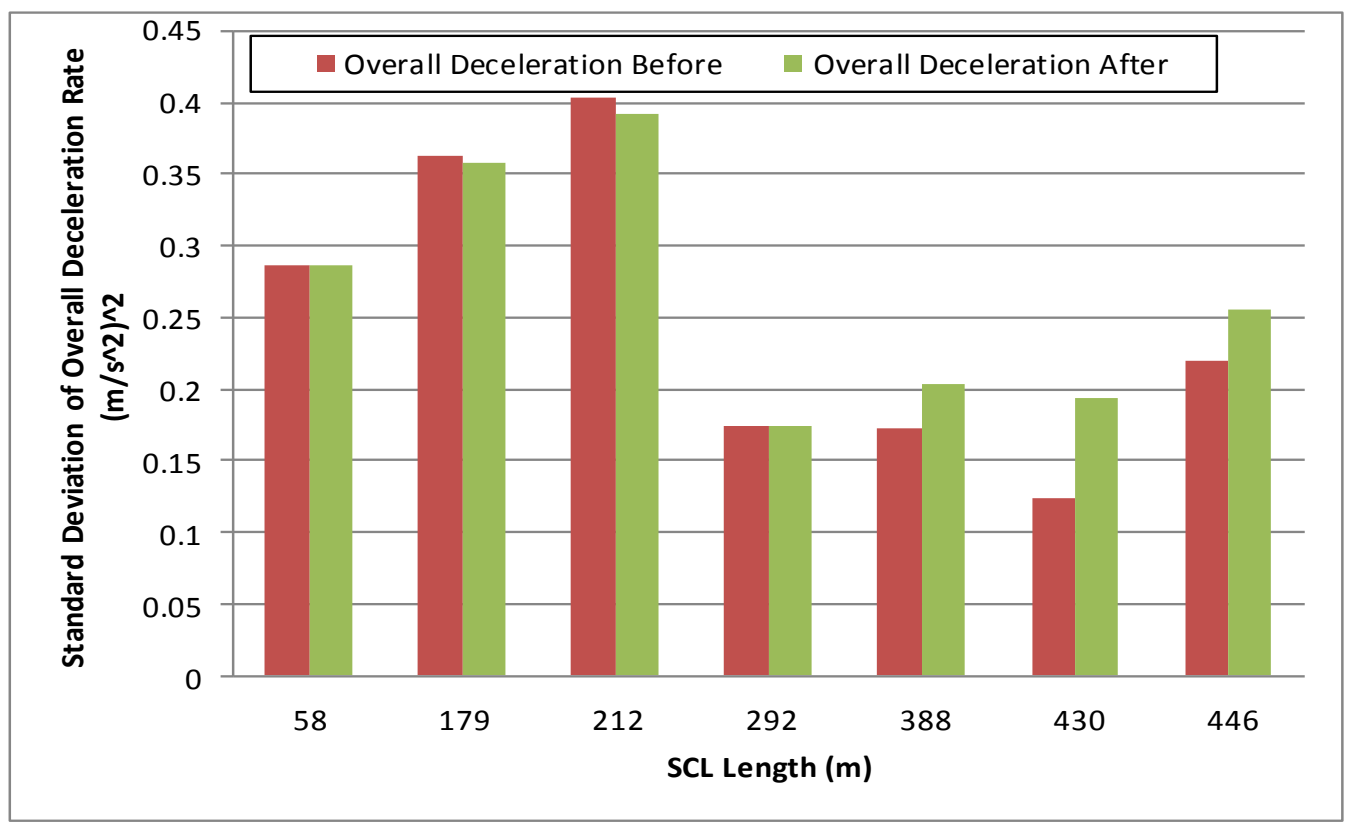

Figure 4.7: Standard Deviation of Overall Deceleration Rate before and after Reprocessing the Data. 


\subsubsection{Correlation between Variables}

The correlation coefficients between variables changed due to reprocessing the data. To summarize this change, the correlation coefficients of all data combined before and after reprocessing and their corresponding $p$-values are shown in Tables 4.6and 4.7.

Table 4.6: Summary of Correlation Coefficients and Corresponding $\boldsymbol{p}$-values before Reprocessing.

\begin{tabular}{|c|c|c|c|c|}
\hline \multicolumn{5}{|c|}{ Correlation Coefficient $(\rho)$ and Corresponding $p$-values } \\
\hline & $v_{i}$ & $v_{f}$ & $d^{*}$ & $U^{*}$ \\
\hline$v_{i}$ & 1 & $0.906(<0.001)$ & $-0.273(<0.001)$ & $0.385(<0.001)$ \\
\hline$v_{f}$ & $0.906(<0.001)$ & 1 & $-0.550(<0.001)$ & $0.361(<0.001)$ \\
\hline$d^{*}$ & $-0.273(<0.001)$ & $-0.550(<0.001)$ & 1 & $-0.460(<0.001)$ \\
\hline$U^{*}$ & $0.385(<0.001)$ & $0.361(<0.001)$ & $-0.460(<0.001)$ & 1 \\
\hline
\end{tabular}

* Before reprocessing utilization $(U)$ and deceleration $(d)$ rates were calculated based on total travelled distance to be able to compare the old parameters to reprocessed parameters.

Table 4.7: Summary of correlation Coefficients and Corresponding P-values after Reprocessing

\begin{tabular}{|c|c|c|c|c|}
\hline \multicolumn{5}{|c|}{ Correlation Coefficient $(\rho)$ and Respective $p$-values } \\
\hline & $v_{i}$ & $v_{f}$ & $d$ & $U$ \\
\hline$v_{i}$ & 1 & $0.914(<0.001)$ & $-0.209(<0.001)$ & $-0.007(0.429)$ \\
\hline$v_{f}$ & $0.914(<0.001)$ & 1 & $-0.487(<0.001)$ & $-0.048(0.112)$ \\
\hline$d$ & $-0.209(<0.001)$ & $-0.487(<0.001)$ & 1 & $-0.262(<0.001)$ \\
\hline$U$ & $-0.007(0.429)$ & $-0.048(0.112)$ & $-0.262(<0.001)$ & 1 \\
\hline
\end{tabular}

After reprocessing, utilization $(U)$ and deceleration $(d)$ rates were calculated based on deceleration distance.

As explained earlier in Section 4.3.4, deceleration distances are different from total travelled distances, specifically for long SCLs which reduced the utilization rates. Also, this slightly affected the overall deceleration rate as the total time and distance spent on the SCL decreased (increasing trend of time and distance to decelerate) as 
discussed in Section 4.3.1. Initial speeds were very close to the diverge speeds, while final speeds did not change. Due to these changes, correlation coefficients between utilization rate and rest of the variables were affected (last column and row). There is no statistically significant correlation between utilization rate and either initial or final speeds after reprocessing the data, as indicated by the $p$-values. The rest of the coefficients did not change considerably (Tables 4.6 and 4.7). 


\section{Chapter Five}

\subsection{Probabilistic Methodology}

In this chapter, a description of the attempted methods to quantify the operational performance of SCLs is presented. Explanation for each method regarding how the methodology is carried out, strengths and/or weaknesses of each approach is covered as well. Estimating PNC for each site is presented in Chapter 6.0 using the methodologies explained in this chapter once for each site's dataset separately and using the dataset of all sites combined data as well. The probabilistic approaches followed in this study are:

- First Order Second Moment (FOSM).

- Monte Carlo Simulation (MCS).

- First Order Reliability Method (FORM).

FOSM calculations were accomplished using Microsoft Excel, while MCS and FORM were completed using Matlab. Excel is commonly used for simple and intermediate statistical calculations, while Matlab is used for more complex mathematical calculations involving simulation and statistical analysis. The developed Matlab scripts could be found in Appendices 9 and 10.

Section 5.1 presents an introduction to reliability analysis. In Section 5.2, the formulated limit state function used for the proposed probabilistic methods is described. Section 5.3 presents the first probabilistic approach, which is FOSM methodology. Then, Section 5.4 presents the second methodology, which is MCS. Finally, Section 5.5 covers the last statistical technique, which is FORM. 


\subsection{Reliability Analysis}

Reliability analysis is a method of evaluating the performance of a system given a sample with known characteristics and confidence level. Reliability analysis simply evaluates the difference between the demand " $D$ " and the supply " $S$ " sides of a system. If demand exceeds supply the system would fail. Demand and supply could be any functions that describe the system's behaviour. To evaluate the system, a limit state function ' $G$ ', which represents difference between supply and demand, is defined as:

$$
\boldsymbol{G}=\boldsymbol{S}-\boldsymbol{D}
$$

Then, " $G$ " is evaluated using probabilistic approaches. Evaluation approaches could be numerical or analytical, exact or approximate depending on the complexity of the limit state function. The probability of demand exceeding supply is referred to in many engineering fields as the probability of failure $(\mathrm{POF})$, probability of hazard $(\mathrm{POH})$, or probability of noncompliance (PNC). As the probability of demand exceeding supply decreases, the likelihood of system failure decreases.

To be able to apply reliability analysis to any engineering system, information about the system's failure modes and variables describing the demand and supply sides of the system should be known. For example, in structural engineering, demanded load of a beam could be represented by the maximum load the beam would be exposed to, while supply would be the function representing the beam's strength or capacity. Similarly, in highway engineering, supply could be available horizontal or vertical curve radius, while demand could be a function representing the demanded radius by the road users. In the two previous examples, failure mode is demand exceeding supply. 
Researchers in fields like structural engineering describe the probability of demand exceeding supply as probability of failure. The reason is that if demand exceeds the supply, the system fails or becomes unusable (developing cracks, deformation ...). On the other hand, in highway engineering systems, when demand exceeds supply, other conditions have to be met and/or no evasive actions are to be performed by drivers for the system to fail. For example, a demand stopping sight distance (SSD) that exceeds supply for a driver at a specific location would only mean a collision if an object exists when this driver passes this location (Sarhan \& Hassan, 2008). For that reason, this probability is called probability of hazard ( $\mathrm{POH})$ or probability of noncompliance (PNC). Many researchers use different terms to describe the condition of demand exceeding supply and it could be argued that this probability could be expressed based on the consequences of exceeding the supply by the demand. The term probability of noncompliance (PNC) will be used to describe the probability of demand exceeding supply since accommodating maneuvers could be performed by the SCL or mainline drivers when demand exceeds supply.

As mentioned earlier, reliability analysis could be conducted using many probabilistic techniques. The following is a list of some of the techniques used in reliability analysis (Mahadevan \& Haldar, 2000):

- Direct Integration of the limit state function: an exact method applied for simple limit state functions.

- First Order Second Moment (FOSM), which is a simple, truncated Taylor series to estimate the distribution of the supply, demand, or the difference between them. 
- Monte Carlo simulation (MCS), which involves the generation of random numbers to compute the probability distribution of the supply, demand, or the difference between them.

- First and Second Order Reliability Methods (FORM and SORM) are other truncated Taylor series methods that calculate the probability distributions of demand, supply, or the difference between them.

If supply and demand are both normally distributed, independent random variables, then, $G$ (the difference between supply and demand) is normally distributed as well. In this case, PNC can be calculated using the inverse of the standard normal cumulative probability distribution function of a $z$-score equal to the reliability index $(\beta)$. Reliability index is a measure used to relate a desired degree of reliability to the performance of an engineering system. For example, an engineering system with a $\beta$ of 2.5 means that supply will not be exceeded by demand for $99.379 \%$ of the time and PNC would be $0.621 \%$. Generally, using a probabilistic approach helps in:

- Providing an assessment of risk or confidence in the designed system.

- Quantifying under- or over-designing by comparing reliability indices or PNC values to standard or common values used in the field.

\subsection{Limit State Function}

The deceleration distance used by a specific driver, can be calculated based on the initial speed as the driver starts deceleration, final speed at the end of SCL, and the deceleration rate as follows (kinematics relationship): 


$$
D=\frac{v_{i}^{2}-v_{f}^{2}}{2 d}
$$

Where

- $\quad D$ is deceleration distance $(\mathrm{m})$;

$-\quad v_{i}$ is initial speed $(\mathrm{m} / \mathrm{s})$;

- $\quad v_{f}$ is final speed $(\mathrm{m} / \mathrm{s})$; and

$-\quad d$ is deceleration rate $\left(\mathrm{m} / \mathrm{s}^{2}\right)$.

This relationship is based on the assumption that drivers use a constant deceleration rate on the whole SCL available length during their deceleration maneuver. As mentioned previously in Chapter 2.0, according to (Fitzpatrick, et al., 2012), drivers do not use a constant deceleration rate in the deceleration maneuver. However, the assumption of constant deceleration rate was validated visually for the field data used in this study. Speed profiles of all vehicles in each site were drawn and it was observed that the change in successive speeds of individual vehicles is constant. This indicates a constant deceleration rate for the selected limited length SCL sites. Figure 5.1 shows a sample speed profile on Bronson Ave. (W-NS), and Appendix 12 contains the speed profiles for all study sites drawn using Matlab. Therefore, Equation 5.2, which is based on the assumption of constant deceleration rate, was used as the basis to the limit state function for SCL length. 


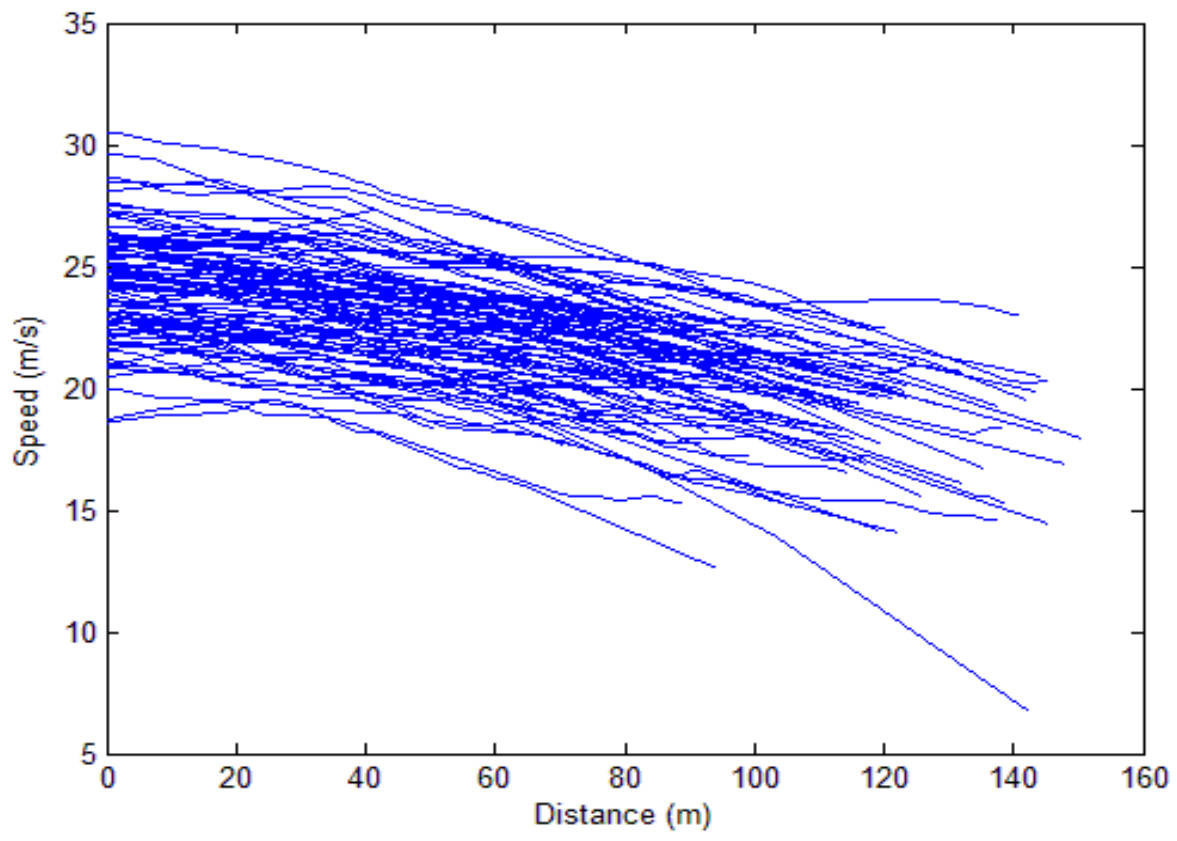

Figure 5.1: Speed Profiles of Individual Vehicles at Bronson Ave. (W-NS)

Equation 5.2 is adjusted for the fact that drivers use a portion of the SCL for deceleration. As mentioned in Chapter 4.0, drivers utilize only a portion of the SCL length to decelerate rather than the whole length. As a result, the deceleration distance in Equation 5.3 could be used to calculate the demand SCL length using the utilization rate $(U)$ developed after reprocessing the data. The limit state function for the demand SCL length $(L)$ can be formulated as:

$$
L=\frac{v_{i}^{2}-v_{f}^{2}}{2 d \times U}
$$

Given that the supply SCL length is constant for a specific site, which is the actual available SCL length $(S L)$, the limit state function for the difference between supply and demand can be formulated as:

$$
G=S L-\frac{v_{i}^{2}-v_{f}^{2}}{2 d \times U}
$$




\subsection{First Order Second Moment (FOSM)}

FOSM is one of simplest statistical techniques that can be used to find the distribution of supply, demand, or limit state function of a system. Mean and variance of the distribution of the function are approximated in a closed form solution using a truncated Taylor series. The function's distribution is estimated using only the first term of Taylor series as follows (Mahadevan \& Haldar, 2000):

$$
\begin{aligned}
& E(Y)=f\left(\mu_{x 1}, \mu_{x 2}, \ldots, \mu_{x n}\right) \\
& \operatorname{Var}(Y)=\sum_{i=1}^{n}\left(\frac{\partial f}{\partial x_{i}}\right)^{2} \sigma_{x i}^{2}+\sum \sum_{i \neq j}^{n} \frac{\partial f}{\partial x_{i}} \frac{\partial f}{\partial x_{j}} \operatorname{cov}\left[x_{i}, x_{j}\right]
\end{aligned}
$$

Where,

- $\quad Y$ is the dependent variable;

- $\quad \mu_{x i}$ and $\sigma_{x i}$ are mean and standard deviation of independent variable $i$;

- $\operatorname{cov}[x i, x j]$ is the covariance between variables $x i, x j$ and equals to $\rho_{x i, x j} \times \sigma_{x i} \times \sigma_{x j}$; and

- $\rho_{x i, x j}$ is the correlation coefficient between variables $x_{i}, x_{j}$.

Equations 5.5 and 5.6 can be applied to the limit state function of demand SCL length in Equation 5.3 to establish the distribution of demand SCL length as follows:

$$
E(L)=\frac{\mu_{v i}^{2}-\mu_{v f}^{2}}{2 \mu_{d} \mu_{U}}
$$




$$
\begin{aligned}
\operatorname{Var}(L)= & \left(\frac{\partial L}{\partial v_{i}}\right)^{2} \sigma_{v i}^{2}+\left(\frac{\partial L}{\partial v_{f}}\right)^{2} \sigma_{v f}^{2}+\left(\frac{\partial L}{\partial d}\right)^{2} \sigma_{d}^{2}+\left(\frac{\partial L}{\partial U}\right)^{2} \sigma_{U}^{2}+2 \frac{\partial L}{\partial v_{i}} \frac{\partial L}{\partial v_{f}} \rho_{v i, v f} \sigma_{v i} \sigma_{v f}+ \\
& 2 \frac{\partial L}{\partial v_{i}} \frac{\partial L}{\partial d} \rho_{v i, d} \sigma_{v i} \sigma_{d}+2 \frac{\partial L}{\partial v_{i}} \frac{\partial L}{\partial U} \rho_{v i, U} \sigma_{V i} \sigma_{u}+2 \frac{\partial L}{\partial v_{f}} \frac{\partial L}{\partial d} \rho_{v f, d} \sigma_{v f} \sigma_{d}+ \\
& 2 \frac{\partial L}{\partial v_{f}} \frac{\partial L}{\partial U} \rho_{v f, U} \sigma_{v f} \sigma_{U}+2 \frac{\partial L}{\partial d} \frac{\partial L}{\partial U} \rho_{d, U} \sigma_{d} \sigma_{U}
\end{aligned}
$$

(Equation 5.8)

Where,

- $E(L)$ is expected or mean SCL length (m);

- $\quad \mu_{v i}$ is mean initial speed $(\mathrm{m} / \mathrm{s})$;

$-\quad \mu_{v f}$ is mean final speed $(\mathrm{m} / \mathrm{s})$

- $\mu_{d}$ is mean deceleration rate $\left(\mathrm{m} / \mathrm{s}^{2}\right)$;

- $\mu_{U}$ is mean utilization rate;

$-\frac{\partial L}{\partial v_{i}}=\frac{v_{i}}{d \times U}$

$-\frac{\partial L}{\partial v_{f}}=-\frac{v_{f}}{d \times U}$

$-\frac{\partial L}{\partial d}=-\frac{v_{i}{ }^{2}-v_{f}{ }^{2}}{2 d^{2} \times U} ;$ and

$-\frac{\partial L}{\partial U}=-\frac{v_{i}{ }^{2}-v_{f}{ }^{2}}{2 d \times U^{2}}$.

Using the mean and standard deviation of the input parameters $\left(v_{i}, v_{f}, U\right.$, and $\left.d\right)$ and correlation coefficients taken from Table 4.1, demand length at each site could be found. Finally, PNC, or the probability of demand exceeding supply, could be estimated using the distribution of demand SCL length $(L)$ and the available length at each site $(S L)$. As all variables follow a normal distribution, demand length $E(L)$ was assumed to follow a normal distribution. It should be noted that the limit state function's nonlinearity could cause the output's distribution (demand length) to follow a distribution different from the 
input parameters normal distribution. This assumption of normality was verified later after applying MCS discussed in Section 5.4 by graphing the frequency of the demand length shown in Appendix 13. Using the cumulative probability density function of $E(L)$, PNC could be found as follows:

$P N C=1-\phi^{-1}\left(\frac{S L-E(L)}{\sqrt{\operatorname{Var}(L)}}\right)$

(Equation 5.9)

Where,

$\phi^{-1}$ is inverse cumulative distribution function of a standard normal distribution.

In Equation 5.9, the difference between supply and demand divided by the standard deviation of demand $L$ is basically called the $z$-score or $\beta$. This $z$-score is the number of standard deviations an observation is above or below the mean.

As shown in the derivation, FOSM is relatively a simple analytical technique with a closed-form solution. However, it has many limitations. First, it suffers lack of invariance of the solution relative to the formulation of the performance function (Kaiser \& Dickman, 1962). In other words, the estimated PNC may differ depending on how the limit state function is formulated. Also, FOSM is applicable only for small to moderately nonlinear systems (Dettinger \& Wilson, 1981). Therefore, if the model is highly nonlinear, the results of the model would be affected. In addition, FOSM was shown to overestimate the variance of the function (Hassan, et al., 2012), which would in turn impact the estimated value of PNC. 


\subsection{Monte Carlo Simulation (MCS)}

In MCS, parameters of the limit state function are treated as uncertain variables, each follows a specific distribution. Random values for each uncertain variable are generated according to their respective probability distributions, and the model describing the system is executed for each random combination of the input parameters. The results of these simulation runs establish the distribution of the limit state function. The accuracy of this distribution depends on the number of simulation runs performed and the adequacy of the assumed parameter distribution.

It was observed that the deceleration rate $(d)$ has high correlation coefficients that are statistically significant at 5\% level of significance to all other independent variables at most sites $\left(v_{i}, v_{f}\right.$, and $U$ ) (Appendix 3). Therefore, only $v_{i}, v_{f}$, and $U$ were taken as independent variables to be assigned randomly to each driver in the simulation. The mean and standard deviation of these variables and correlation coefficients were taken from Table 4.1 corresponding to the site being simulated or for all sites combined. The variable (d) was assigned to each driver using the following regression equation:

$$
\mathrm{d}= \begin{cases}a_{1}+a_{2} v_{i}+a_{3} v_{f}+a_{4} U & \text { Data for a specific site } \\ a_{1}+a_{2} v_{i}+a_{3} v_{f}+a_{4} U+a_{5} S L & \text { Data for all sites combined }\end{cases}
$$

(Equation 5.10)

Where, $a_{\mathrm{i}}$ are regression coefficients.

Table 5.1 shows the linear regression result for the dataset of all sites combined. It should be noted that using all sites combined includes parameters $\left(v_{i}, v_{f}\right.$, and $\left.U\right)$ that belong to different SCLs lengths $(S L)$, therefore, the supply length was taken as a variable 
in predicting the deceleration rate on the SCL as shown in Equation 5.10. As shown in Table 5.1, the actual or supply SCL length $(S L)$ is a significant variable affecting the individual driver's deceleration rate when regression is carried out for the dataset of all sites combined. Using the assumptions of linearity of the relationship between variables, error independence (having no serial correlation), error homoscedasticity (having constant variance for the errors), and error normality distribution, linear regression analysis was performed using the individual vehicle data. Detailed results of the regression analysis are presented in Appendix 6, and the regression coefficients are shown in Table 5.2.

Table 5.1: Linear Regression for the Dataset of All Sites Combined

All Sites Combined

\begin{tabular}{|lrl|}
\hline \multicolumn{2}{|c|}{ Regression Statistics } & \multirow{2}{*}{} \\
\cline { 1 - 2 } Multiple R & 0.921 & \\
R Square & 0.849 & \\
Adjusted R Square & 0.848 & \\
Standard Error & 0.150 & \\
Observations & 638 & \\
\hline Parameter & Coefficients & $P$-value \\
\hline Intercept & 0.547 & $<0.001$ \\
Initial Speed & 0.189 & $<0.001$ \\
Final Speed & -0.169 & $<0.001$ \\
Utilization Rate & -0.835 & $<0.001$ \\
SCL Length & -0.002 & $<0.001$ \\
\hline
\end{tabular}


Table 5.2: Regression Coefficients for Prediction of Individual Vehicle's Deceleration

Rate.

\begin{tabular}{lcccccc}
\hline SCL Name & $a_{1}$ & $a_{2}$ & $a_{3}$ & $a_{4}$ & $a_{5}$ & $R^{2}$ \\
\hline Island Park Drive (E-N) & 0.040 & 0.557 & -0.530 & -0.944 & --- & 0.940 \\
Parkdale Avenue (W-NS) & 0.046 & 0.313 & -0.273 & -2.215 & --- & 0.963 \\
Bronson Avenue (W-NS) & -0.289 & 0.225 & -0.162 & -2.024 & --- & 0.927 \\
Moodie Drive (W-NS) & 0.060 & 0.184 & -0.173 & -0.693 & --- & 0.929 \\
Terry Fox Drive (E-NS) & -0.090 & 0.146 & -0.127 & -0.798 & --- & 0.882 \\
Carp Road (E-NS) & 0.104 & 0.128 & -0.118 & -0.639 & --- & 0.878 \\
Terry Fox Drive (W-NS) & -0.180 & 0.152 & -0.131 & -0.801 & --- & 0.843 \\
All Sites Combined & 0.547 & 0.189 & -0.169 & -0.835 & -0.002 & 0.849 \\
\hline
\end{tabular}

All regression coefficients are significant at 5\% level of significance.

The linearity of the relationship between variables and error homoscedasticity were validated for the datasets of each site separately and all sites combined by drawing the line of equality between the observed deceleration rates at each site and the predicted values using Equation 5.10. Figure 5.2 shows this test for the data of all sites combined, and the complete analysis is available in Appendix 7. To test errors' independence, plots between the observations and the residuals were generated similar to the example shown in Figure 5.3. Finally, to test the errors' normality distribution, histogram of the residuals was drawn as shown in Figure 5.4. The diagnostics indicate that all deceleration regression models performed for the datasets of each site separately and all sites 
combined do not violate the linear regression assumptions. Complete diagnostics of the regression models for each site and for all sites combined are available in Appendix 7.

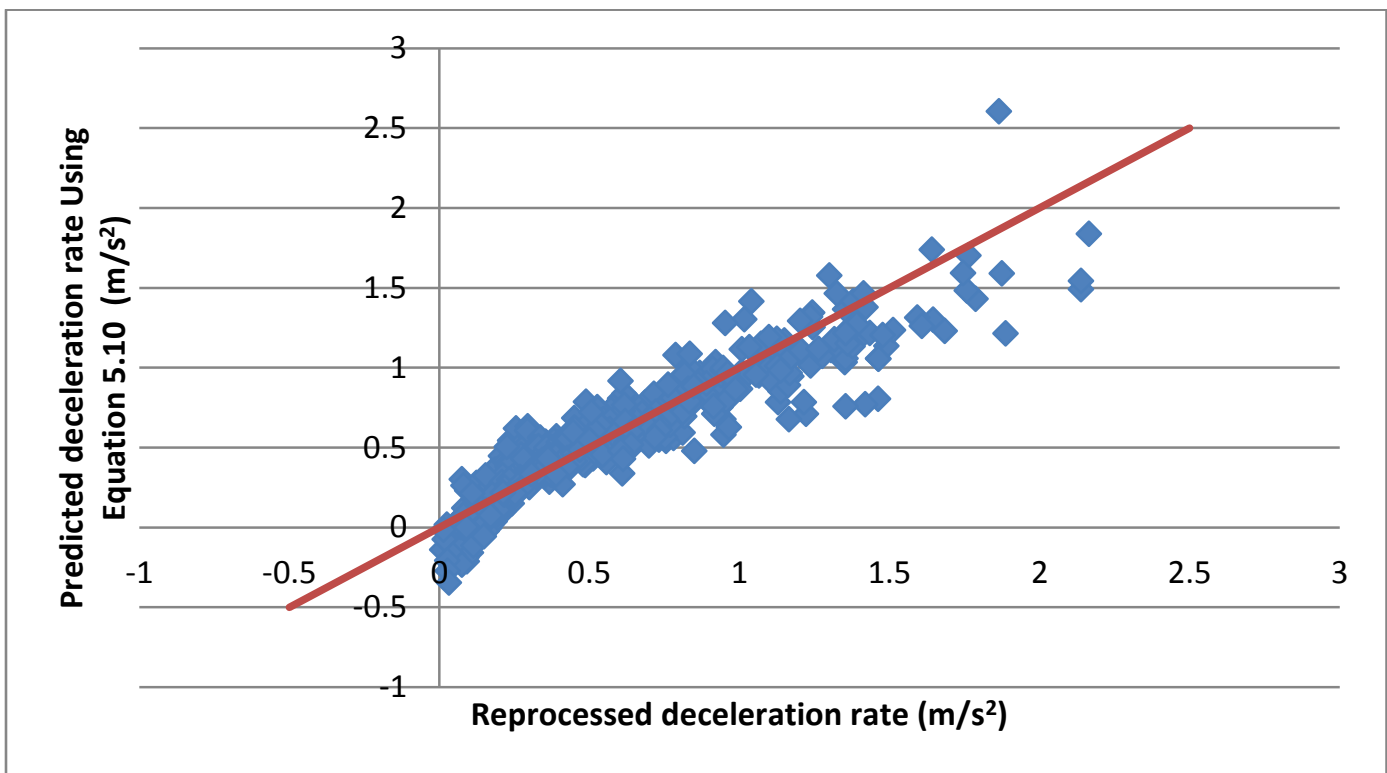

Figure 5.2: Line of Equality between Reprocessed Deceleration Rate and Predicted Deceleration Rate Using Equation 5.10 for All SCLs Combined.

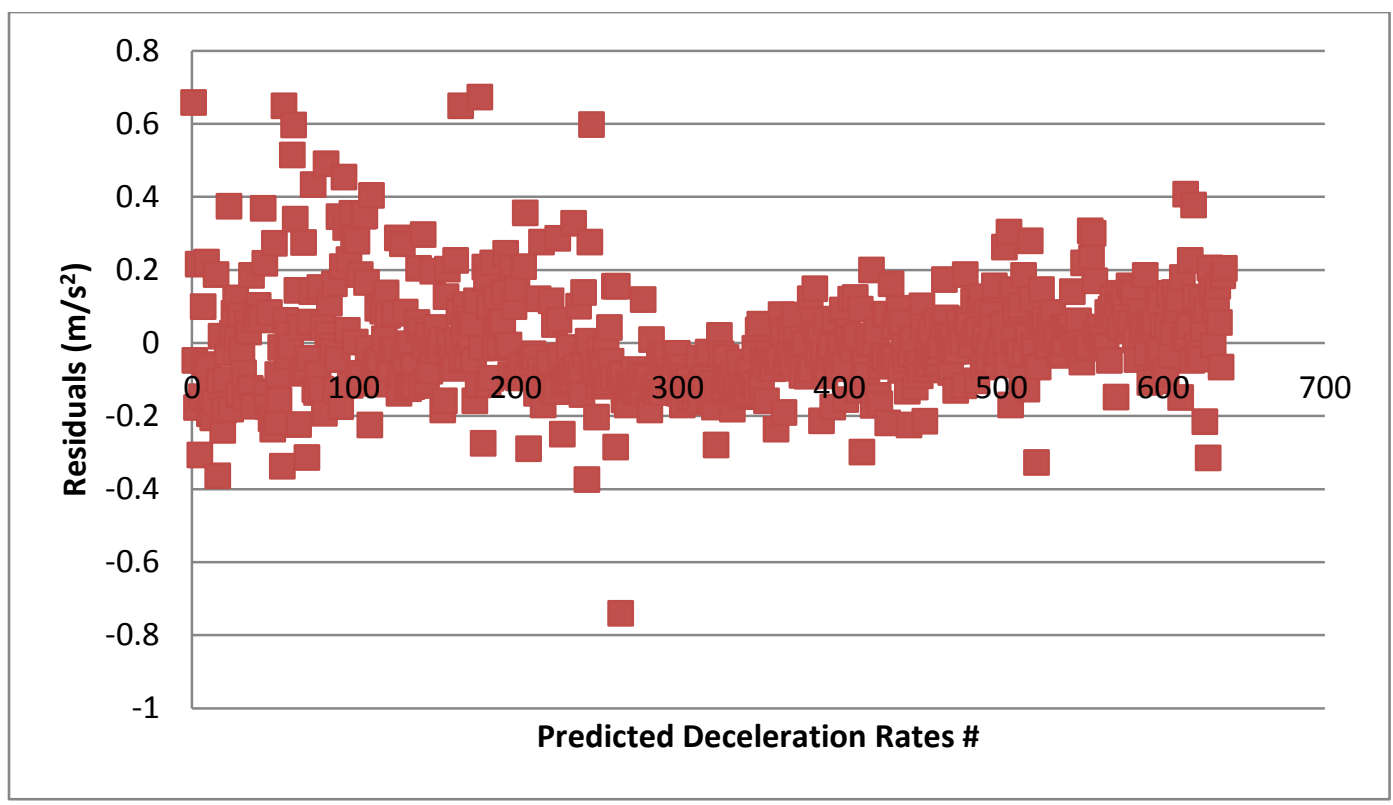

Figure 5.3: Residuals Plot for Each Predicted Deceleration Rate Using Equation 5.10 at

\section{All Sites Combined}




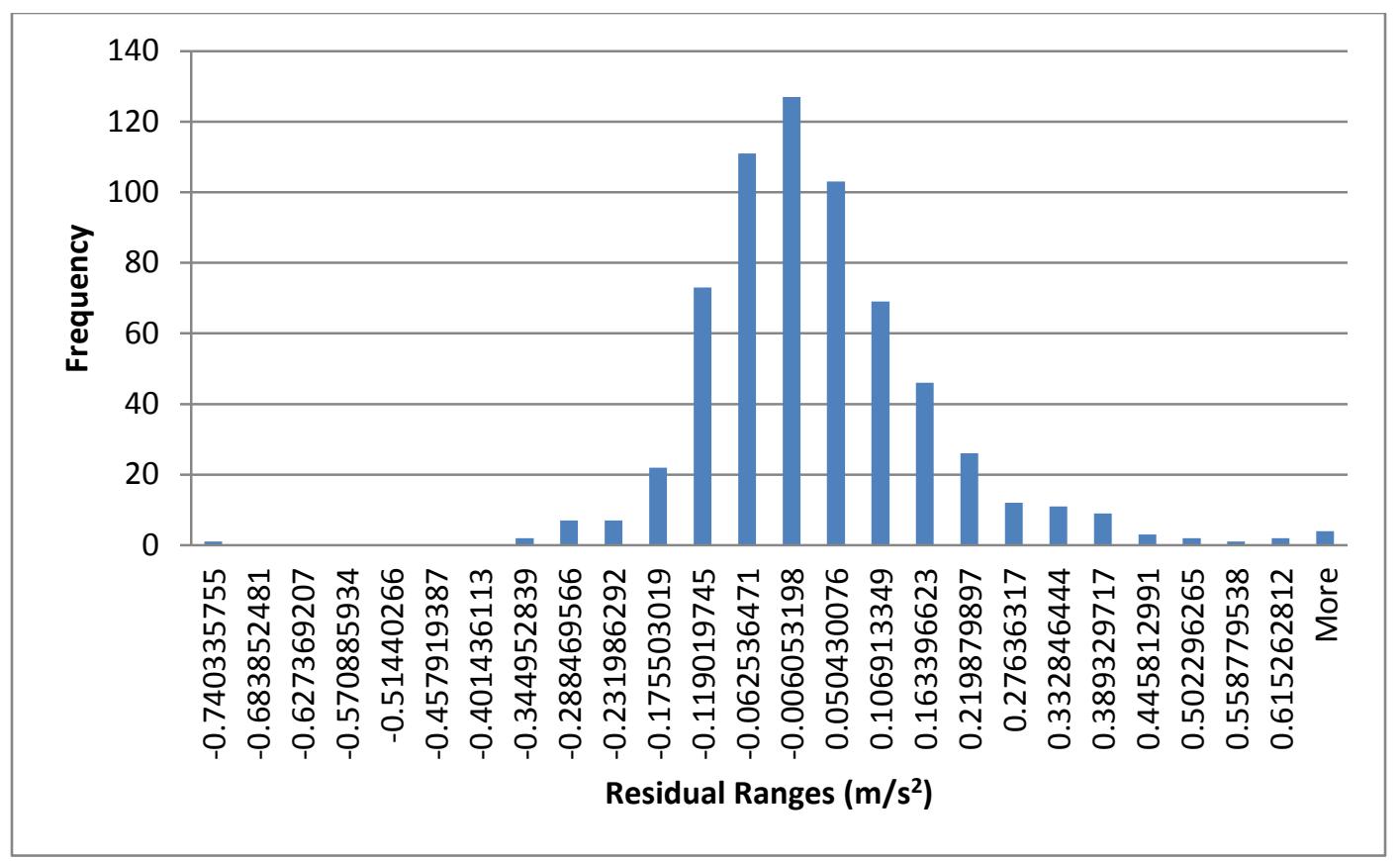

Figure 5.4: Residuals Histogram for All Sites Combined

To apply MCS to the limit state function in Equation 5.4, the mean and standard deviation of each variable, along with the correlation coefficient between each two variables, were first defined based on field data. Then, uncorrelated random numbers in the range $[0,1]$ were generated for each of the independent variables in the limit state function. The uncorrelated random numbers were then converted into random, correlated numbers in the range $[0,1]$ using Cholesky's decomposition method (Mahadevan \& Haldar, 2000). Cholesky's decomposition is a triangular decomposition of a matrix $C$, which finds a triangular matrix $B$ that if multiplied by its transpose will lead back to the matrix $C$; thus, $C=B^{T} B$. Choesky's decomposition of the correlation matrix was multiplied by the uncorrelated random numbers leading to the correlated random numbers in the range $[0,1]$. Then, using inverse of the normal cumulative distribution function, the correlated random numbers in the range $[0,1]$ were converted to normally 
distributed parameters based on the mean and standard deviation of each variable $\left(v_{i}, v_{f}\right.$, and $U$ ). Values were screened to remove all combinations that would create any impossible or unrealistic simulation scenario. The following two conditions represent impossible conditions and corresponding scenarios were removed:

- Negative initial and diverge speeds.

- Utilization rate exceeding 1 or below 0 .

Then deceleration values were generated based on the linear regression shown in Equation 5.10 using the coefficients in Table 5.2. Length is calculated for each simulated vehicle based on Equation 5.4. To make sure that outliers are removed, scenarios with the following conditions were also removed from the simulation:

- Difference between final speed and initial speed that is below 0 as this violates the deceleration condition.

- Values that were outside $95 \%$ of the normal distribution curve (i.e. values exceeding 1.96 standard deviations) of the following:

- Deceleration rates.

- Utilization rates.

○ Difference between initial and final/initial speed.

- Utilization rates that were less than the minimum at each site when using individual sites data; and utilization rates less than the minimum at all sites when using all data combined. This is to remove utilization rates that would be too low based on the available field data. For instance, if the generated utilization rate was 0.03 and the minimum in Terry Fox Drive W-NS is 0.05 , the line that contains the 0.03 value of utilization rate 
would be removed. This in turn truncates the distribution of utilization rates from the left side resulting in a truncated normal distribution. Right side truncation, i.e. truncating values that exceed the maximum at each site was not performed as high utilization rates do not represent an unwanted scenario. On the other hand, an extremely low $U$ would be a driver who diverged within the gore proximity.

- Deceleration rates that were less than the minimum at each site when using individual sites data; and deceleration rates that were less than the minimum at all sites when using all data combined. This step is done to remove generated combinations of initial and final speeds and utilization rates that do not represent the study sites. Similar to utilization rates, the distribution of deceleration rates is truncated from the left side resulting in a truncated normal distribution. Right side truncation, i.e. truncating values that exceed the maximum at each site was not performed as high deceleration rates do not represent an unwanted scenario. On the other hand, a very low $d$ means one of the following unwanted scenarios: drivers entered the SCL at a low initial speed and left with a low final speed, drivers entered with high initial speed and left with high final speed, and/or drivers utilized a very low portion of the SCL.

Finally, PNC in MCS is calculated as the number of failures $(L>S L)$ divided by the number of runs. These steps were followed using the dataset of each site combined. 
When the evaluation of the dataset of all sites combined was carried out, the following distributions were used to represent the data:

- Mean and standard deviation of initial speed at long sites, $\mu_{v i \_l o n g}=29.085$ $\mathrm{m} / \mathrm{s}$ and $\sigma_{v i}$ long $=2.472 \mathrm{~m} / \mathrm{s}$.

- Mean and standard deviation of final speed at each site.

- Mean and standard deviation of utilization rate at all sites combined.

Mean and standard deviation of initial speed at long SCL sites were used to avoid the drivers' deceleration behaviour on the FRL as indicated in Section 4.2.2. The mentioned distributions of initial speed, final speed, and utilization rate were used as distributions for the random generated independent parameters. As deceleration rate is predicted for each driver during the MCS procedure, it was necessary to avoid the issue of drivers decelerating on the FRL, which is why the above distributions were used for the generated parameters. These distributions were only followed in the simulation using the dataset of all sites combined. On the other hand, it was not possible to avoid the deceleration condition on the FRL when using the analytical methods (FOSM and FORM) since the deceleration could occur on the FRL, which is the reason mean and standard deviations of the independent parameters were used. The condition of drivers decelerating on the FRL could not be avoided in analytical methods as it was not clear if embedding a function inside the demand distribution would affect the methodology.

It should be noted that attempts to predict mean and standard deviation of each parameter were carried out. Trials were carried to model the distributions (mean and standard deviation) of final speed and utilization rate. However, there were no significant 
relations for mean or standard deviation of final speed and utilization rate. Since no independent parameters had any relationship, it was decided to use the distributions that would reflect the sites based on the data as discussed.

A Matlab script was developed to perform the MCS procedure explained in this section (Appendix 9). The simulation started with creating 150,000 combinations of random input parameters. After screening the combinations for unrealistic scenarios and outliers based on the value of each independent parameter, the final number of simulation runs using the datasets for each site separately ranged from 61,756 to 81,628 for each site's simulation and 78,227 to 106,435 runs using the dataset for all sites combined. Summary for the number of runs on each site could be found in Section 6.2. Error of this methodology is inversely proportional to the number of runs as shown in Equation 5.11 (Mahadevan \& Haldar, 2000).

$$
S E=\frac{\sigma}{N^{0.5}}
$$

Where,

$\sigma$ is the standard deviation of the MCS simulation and $N$ is the number of runs.

The results discussed in Section 6.2 show that the error of this methodology is very low.

\subsection{First Order Reliability Method (FORM)}

FORM is another reliability analysis method used to incorporate the uncertainty of the supply and demand. PNC is computed directly by evaluating the limit state function for the difference between supply and demand. Therefore, in the case of SCL length, the limit state function in FORM is the one given in Equation 5.4. FORM has been developed to address some of the technical difficulties in FOSM mentioned in 
Section 5.3. Still, this method is a Taylor series expansion but about a point that is on the failure surface of the performance function (Singh, et al., 2007). This failure surface is the surface constrained to a limit state function equal to zero. The point of expansion is determined as the point on the failure surface at the shortest distance to the origin (Singh, et al., 2007). This distance from the origin to the expansion point is a measure of reliability, called reliability index, $\beta$.

The methodology used in this study to determine the reliability index and PNC is called the Ellipsoid method. This method was developed by Low \& Tang (1997) who showed that $\beta$ can be estimated by minimizing a quadratic form subject to a specific constraint. Mathematically, the solution can be formulated in a matrix form as follows (Low \& Tang, 1997):

$\boldsymbol{\beta}=\boldsymbol{m i n}_{X \in F} \sqrt{(X-\boldsymbol{\mu})^{T} C^{-1}(X-\boldsymbol{\mu})}$, subject to the constraint: $\boldsymbol{G}=\mathbf{0}$ (Equation 5.12)

Where,

- $\quad X$ is a vector of random variables;

- $\quad \mu$ is a vector of corresponding mean values;

- $\quad C$ is the covariance matrix of the variables;

- $F$ is the failure region; and

- $G$ is the limit state function in Equation 5.4.

In applying the methodology to the limit state function in Equation 5.4, the vectors of random variables and corresponding mean values are as follows: 


$$
X=\left[\begin{array}{c}
v_{i} \\
v_{f} \\
d \\
U
\end{array}\right] \rightarrow \mu=\left[\begin{array}{l}
\mu_{v_{i}} \\
\mu_{v_{f}} \\
\mu_{d} \\
\mu_{U}
\end{array}\right]
$$

The covariance matrix can be formulated as:

$$
C=\left[\begin{array}{cccc}
\sigma_{v i}^{2} & \rho_{v i, v f} \sigma_{v i} \sigma_{v f} & \rho_{v i, d} \sigma_{v i} \sigma_{d} & \rho_{v i, U} \sigma_{v i} \sigma_{U} \\
\rho_{v f, v i} \sigma_{v f} \sigma_{v i} & \sigma_{v f}^{2} & \rho_{v f, d} \sigma_{v f} \sigma_{d} & \rho_{v f, U} \sigma_{v f} \sigma_{U} \\
\rho_{d, v i} \sigma_{d} \sigma_{v i} & \rho_{d, v f} \sigma_{d} \sigma_{v f} & \sigma_{d}^{2} & \rho_{d, U} \sigma_{d} \sigma_{U} \\
\rho_{U, v i} \sigma_{U} \sigma_{v i} & \rho_{U, v f} \sigma_{U} \sigma_{v f} & \rho_{U, d} \sigma_{U} \sigma_{d} & \sigma_{U}^{2}
\end{array}\right]
$$

Substituting in to Equation 5.12, $\beta$ will be equal to

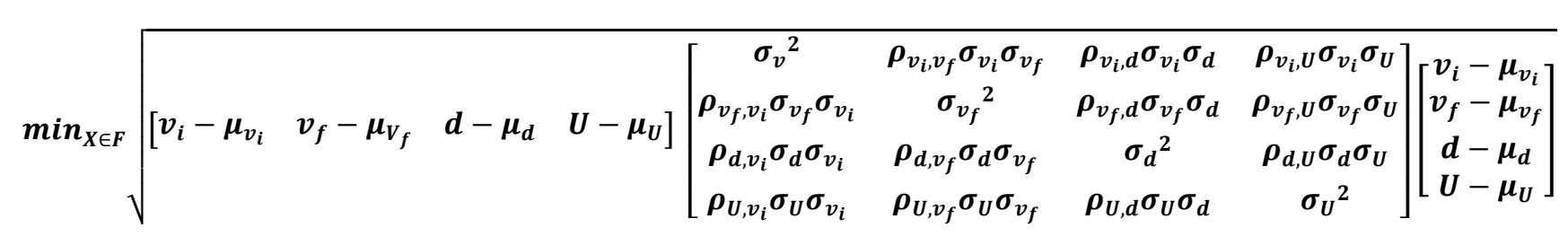

(Equation 5.15)

The reliability index $\beta$ can then be found by substituting the variables in Equation 5.15 and solving for a minimum value with the minimization constraint (Equation 5.16):

$$
G=S L-\frac{v_{i}^{2}-v_{f}^{2}}{2 d \times U}=0
$$


Then, using the inverse cumulative distribution function of a normal distribution, PNC is calculated as shown in Equation 5.17.

$P N C=1-\phi^{-1}(\beta)$ (Equation 5.17)

FORM is accurate enough when the curvature radius of failure surface is large and small number of random variables is present (Zhao \& Ono, 1999). However, when the failure surface is highly nonlinear, Second Order Reliability Method (SORM) is more accurate than FORM. One of the limitations of the followed approach is that it suffers from having a maximum PNC of 0.5. This maximum value results from the physical interpretation of the used methodology. Reliability index, $\beta$, is found by calculating the minimum distance from the center of the formulated ellipsoid to the failure surface. If that distance is 0 , which is the minimum value possible under the square root, $\mathrm{PNC}$ will be at the maximum possible value in this method, which is 0.5 .

A Matlab script was developed to perform the FROM procedure explained in this section. First the dataset means, standard deviations, correlation coefficients were extracted from an Excel sheet. After that, the limit state function was defined. Then, using a minimization range of two standard deviations above or below the mean, the minimization equation (Equation 5.15) was applied with the condition of a limit state function equal to zero. Finally, PNC was calculated using the inverse cumulative distribution of a normal function (Equation 5.17). The Matlab script could be found in Appendix 10. The methodology was applied once using the dataset of each site and another time using the dataset of all sites combined and the results are followed and discussed in Chapter 6.0. 


\section{Chapter Six}

\subsection{Results and Discussion}

This chapter covers the results of the three attempted probabilistic methods. Results are summarized as PNC values, which are compared later in this chapter to the available collision data as a way to explore the premise of the methodology. The first section in this chapter covers the results of FOSM using the dataset of each site and all sites combined. Similarly, Sections 6.2 and 6.3 will cover MCS and FORM, respectively. A comparison between the used methods is followed in Section 6.4. Finally, the trend of change in collision frequency with PNC values is examined to indicate whether a potential relationship between PNC and collisions exists.

\subsection{First Order Second Moment (FOSM)}

As stated in Section 5.3 FOSM was performed using each site's data and using all sites combined data. Input data from Table 4.1 were used in Equations 5.7 and 5.8 in order to find the demand length. Then, Equation 5.9 was used to find the PNC. Table 6.1 provides a summary of the results using the dataset of each site. 
Table 6.1: Results of FOSM Using the Dataset of Each Site.

\begin{tabular}{|c|c|c|c|c|c|}
\hline SCL & $\begin{array}{c}\text { Available } \\
\text { Length } S L \\
(\mathrm{~m})\end{array}$ & $\begin{array}{c}\text { Mean Demand }(L) \\
(\mathrm{m})\end{array}$ & $\begin{array}{c}\text { Standard } \\
\text { Deviation of } L \\
(\mathrm{~m})\end{array}$ & $\beta$ & PNC \\
\hline $\begin{array}{c}\text { Island Park } \\
\text { Drive (E-N) }\end{array}$ & 58 & 59.644 & 34.501 & -0.048 & 0.519 \\
\hline $\begin{array}{c}\text { Parkdale } \\
\text { Avenue (W- } \\
\text { NS) }\end{array}$ & 179 & 180.272 & 73.126 & -0.017 & 0.507 \\
\hline $\begin{array}{c}\text { Bronson } \\
\text { Avenue (W- } \\
\text { NS) }\end{array}$ & 212 & 211.311 & 86.880 & 0.008 & 0.497 \\
\hline $\begin{array}{c}\text { Moodie Drive } \\
\text { (W-NS) }\end{array}$ & 292 & 277.475 & 157.571 & 0.092 & 0.463 \\
\hline $\begin{array}{c}\text { Terry Fox } \\
\text { Drive (E-NS) }\end{array}$ & 388 & 361.501 & 268.022 & 0.099 & 0.461 \\
\hline $\begin{array}{c}\text { Carp Road (E- } \\
\text { NS) }\end{array}$ & 430 & 376.264 & 330.748 & 0.162 & 0.435 \\
\hline $\begin{array}{c}\text { Terry Fox } \\
\text { Drive (W-NS) }\end{array}$ & 446 & 383.550 & 358.480 & 0.174 & 0.431 \\
\hline
\end{tabular}

It can be noticed that due to the high standard deviation of expected demand $L$, this approach results in high PNC values for long sites (compared to MCS in the following section). The main reason of such PNC values is drivers' adjusted behaviour to account for different SCL lengths.

Repeating the same methodology, PNC values were found for each site using the dataset of all sites combined available in Table 4.1. When the model was executed, the expected demand $L$ was found as $235.449 \mathrm{~m}$ with a standard deviation of $184.863 \mathrm{~m}$. Based on this result and applying Equation 5.9 for each site, the following PNC values were calculated for each site (Table 6.2). 
Table 6.2: Results of FOSM Using the Dataset of All Sites Combined.

\begin{tabular}{|c|c|c|c|}
\hline SCL & $\begin{array}{c}\text { Available Length } \\
\text { SL (m) }\end{array}$ & $\beta$ & PNC \\
\hline Island Park Drive (E-N) & 58 & -0.960 & 0.832 \\
\hline Parkdale Avenue (W-NS) & 179 & -0.306 & 0.620 \\
\hline Bronson Avenue (W-NS) & 212 & -0.127 & 0.551 \\
\hline Moodie Drive (W-NS) & 292 & 0.305 & 0.380 \\
\hline Terry Fox Drive (E-NS) & 388 & 0.825 & 0.205 \\
\hline Carp Road (E-NS) & 430 & 1.052 & 0.146 \\
\hline Terry Fox Drive (W-NS) & 446 & 1.138 & 0.127 \\
\hline
\end{tabular}

As shown in Table 6.2, and similar to the results using the dataset of each site in Table 6.1, as available SCL length $(S L)$ increases PNC decreases. When using the dataset of all sites combined, the demand length is constant, and therefore the value of PNC at each site depends only on the available SCL length. This explains the difference in trend of changing PNC with SCL length compared to the case of using each site's data.

\subsection{Monte Carlo Simulation (MCS)}

MCS was performed once using the dataset of each site and another time using the dataset of all sites combined as discussed in Section 5.4. Using the developed Matlab script (Appendix 9), PNC was predicted for each site. Tables 6.3 and 6.4 show number of runs after screening, mean demand $L$, standard deviation of $L$, PNC results, and error of demand length for the dataset of each site and for dataset of all sites combined, respectively. 
When each site was evaluated using its own dataset, PNC was based on each site's conditions (initial speed, utilization rate, and final speed). For example, in case of short SCLs, the correlated parameters were generated based on the distribution of initial speed, final speed and utilization rate at the site, as well as this site's regression equation for deceleration rate (Table 5.2). Therefore, this approach considers changing driver behaviour, in terms of selection of initial speed, utilization rate, and deceleration rate, depending on the SCL length. On the other hand, when using the dataset of all sites combined, the distribution (mean and standard deviation) of initial speed at long lanes, final speed at each site, utilization rate of the dataset of all sites combined were used for the generated independent parameters. Deceleration rate was predicted using all sites combined regression equation (Table 5.2). Therefore, the PNC results for dataset of all sites combined reflect the expected PNC values if no deceleration was to occur on the FRL.

As shown in the Tables 6.3 and 6.4, error of MCS is low, which is mainly contributed to the high number of runs. PNC results follow the same trend of FOSM method. As the available length increases, PNC decreases. The reason of the high PNC values for the first three sites, when using the dataset of all sites combined (Table 6.4), is the high difference between initial and final speed as the distribution of initial speeds at long sites was used for the generated correlated initial and final speeds. 
Table 6.3: MCS Results Using the Dataset of Each Site's.

\begin{tabular}{|c|c|c|c|c|c|c|}
\hline SCL & $\begin{array}{c}\text { Available } \\
\text { Length } \\
\text { SL (m) }\end{array}$ & $\begin{array}{c}\text { No of } \\
\text { Runs }\end{array}$ & $\begin{array}{c}\text { Mean } \\
\text { Demand L } \\
(\mathrm{m})\end{array}$ & $\begin{array}{c}\text { Standard } \\
\text { Deviation } \\
\text { L } \\
(\mathrm{m})\end{array}$ & PNC & $\begin{array}{c}\text { Error } \\
(\mathrm{m})\end{array}$ \\
\hline $\begin{array}{c}\text { Island Park } \\
\text { Drive (E-N) }\end{array}$ & 58 & 61,756 & 53.135 & 14.344 & 0.515 & 0.058 \\
\hline $\begin{array}{c}\text { Parkdale } \\
\text { Avenue (W-NS) }\end{array}$ & 179 & 66,168 & 174.255 & 20.788 & 0.582 & 0.081 \\
\hline $\begin{array}{c}\text { Bronson Avenue } \\
\text { (W-NS) }\end{array}$ & 212 & 81,628 & 202.294 & 28.474 & 0.432 & 0.100 \\
\hline $\begin{array}{c}\text { Moodie Drive } \\
\text { (W-NS) }\end{array}$ & 292 & 80,106 & 248.332 & 77.825 & 0.195 & 0.275 \\
\hline $\begin{array}{c}\text { Terry Fox Drive } \\
\text { (E-NS) }\end{array}$ & 388 & 78,224 & 352.800 & 118.846 & 0.252 & 0.425 \\
\hline $\begin{array}{c}\text { Carp Road (E- } \\
\text { NS) }\end{array}$ & 430 & 79,269 & 376.461 & 153.833 & 0.226 & 0.546 \\
\hline $\begin{array}{c}\text { Terry Fox Drive } \\
\text { (W-NS) }\end{array}$ & 446 & 69,538 & 412.523 & 230.164 & 0.248 & 0.873 \\
\hline
\end{tabular}

Table 6.4: MCS Results Using the Dataset of All sites Combined.

\begin{tabular}{|c|c|c|c|c|c|c|}
\hline SCL & $\begin{array}{c}\text { Available } \\
\text { Length } S L \\
(\mathrm{~m})\end{array}$ & $\begin{array}{c}\text { No of } \\
\text { Runs }\end{array}$ & $\begin{array}{c}\text { Mean } \\
\text { Demand } \\
L \\
(\mathrm{~m})\end{array}$ & $\begin{array}{c}\text { Standard } \\
\text { Deviation } \\
L(\mathrm{~m})\end{array}$ & PNC & $\begin{array}{c}\text { Error } \\
(\mathrm{m})\end{array}$ \\
\hline $\begin{array}{c}\text { Island Park } \\
\text { Drive (E-N) }\end{array}$ & 58 & 105,843 & 569.587 & 159.863 & 1 & 0.491 \\
\hline $\begin{array}{c}\text { Parkdale } \\
\text { Avenue (W-NS) }\end{array}$ & 179 & 106,348 & 589.600 & 166.607 & 1 & 0.511 \\
\hline $\begin{array}{c}\text { Bronson Avenue } \\
\text { (W-NS) }\end{array}$ & 212 & 106,435 & 626.960 & 176.218 & 1 & 0.540 \\
\hline $\begin{array}{c}\text { Moodie Drive } \\
\text { (W-NS) }\end{array}$ & 292 & 94,392 & 206.007 & 126.859 & $\begin{array}{c}9 \\
9\end{array}$ & 0.413 \\
\hline $\begin{array}{c}\text { Terry Fox Drive } \\
\text { (E-NS) }\end{array}$ & 388 & 101,978 & 288.956 & 150.279 & $\begin{array}{c}0.235 \\
8\end{array}$ & 0.471 \\
\hline $\begin{array}{c}\text { Carp Road (E- } \\
\text { NS) }\end{array}$ & 430 & 91,675 & 227.327 & 147.000 & $\begin{array}{c}7 \\
7\end{array}$ & 0.486 \\
\hline $\begin{array}{c}\text { Terry Fox Drive } \\
\text { (W-NS) }\end{array}$ & 446 & 78,227 & 207.402 & 132.403 & 5 & 0.473 \\
\hline
\end{tabular}




\subsection{First Order Reliability Method (FORM)}

FORM was applied to each site using the developed script in Appendix 10. The ellipsoid methodology discussed in section 5.5 was applied to calculate PNC values. Table 6.5 and 6.6 show the results when using the dataset of each site and all sites combined, respectively.

Table 6.5: FORM results Using the Dataset of Each Site.

\begin{tabular}{|c|c|c|}
\hline SCL & Available length SL (m) & PNC \\
\hline Island Park drive (E-N) & 58 & 0.5000 \\
\hline Parkdale Avenue (W-NS) & 179 & 0.5000 \\
\hline Bronson Avenue (W-NS) & 212 & 0.4885 \\
\hline Moodie Drive (W-NS) & 292 & 0.3606 \\
\hline Terry Fox Drive (E-NS) & 388 & 0.3590 \\
\hline Carp Road (E-NS) & 430 & 0.2572 \\
\hline Terry Fox Drive (W-NS) & 446 & 0.3221 \\
\hline
\end{tabular}

Table 6.6: FORM results Using the Dataset of Each Site All Sites Combined.

\begin{tabular}{|c|c|c|}
\hline SCL & Available length at Site & PNC \\
\hline Island Park drive (E-N) & 58 & 0.5000 \\
\hline Parkdale Avenue (W-NS) & 179 & 0.5000 \\
\hline Bronson Avenue (W-NS) & 212 & 0.5000 \\
\hline Moodie Drive (W-NS) & 292 & 0.3490 \\
\hline Terry Fox Drive (E-NS) & 388 & 0.2071 \\
\hline Carp Road (E-NS) & 430 & 0.1761 \\
\hline Terry Fox Drive (W-NS) & 446 & 0.1672 \\
\hline
\end{tabular}


As mentioned in Section 5.5, maximum PNC resulting from the methodology used is 0.5 . Therefore, PNC values for short SCLs should be evaluated carefully as their actual PNC could exceed 0.5; however, FORM may show it as 0.5. FORM results follow the same trends of the previous two methods used to evaluate PNC at SCLs. As the available length $(S L)$ increases, PNC decreases.

\subsection{Comparison between FOSM, MCS, and MCS}

When FOSM and MCS were carried out, the values of expected demand $L$ and their respective standard deviations could be found during the process. However, when FORM was carried out, these values were not be generated within the process. Thus, the values for the mean demand $L$ and their standard deviations were compared only for FOSM and MCS. Then, the comparison of PNC values for the three methodologies was carried out. Figure 6.1 shows a comparison of FOSM and MCS with respect to mean demand $L$ using the dataset of each site. Figure 6.2 shows the comparison of the standard deviation using each site's data for FOSM and MCS.

Mean demand length using FOSM is very close to MCS as shown in Figure 6.1. This validates the results of FOSM in estimating demand SCL length. On the other hand, as shown in Figure 6.2, it was found that FOSM methodology overestimates the standard deviations compared to MCS due to the nonlinearity of the limited state function discussed in Section 5.3. This result is similar to the findings of Hassan, Sarhan, \& Salehi (2012). Figures 6.3 and 6.4 show a comparison of the PNC results using the three methodologies (FOSM, MCS, and FORM) using the dataset each site and using the dataset of all sites combined, respectively. 


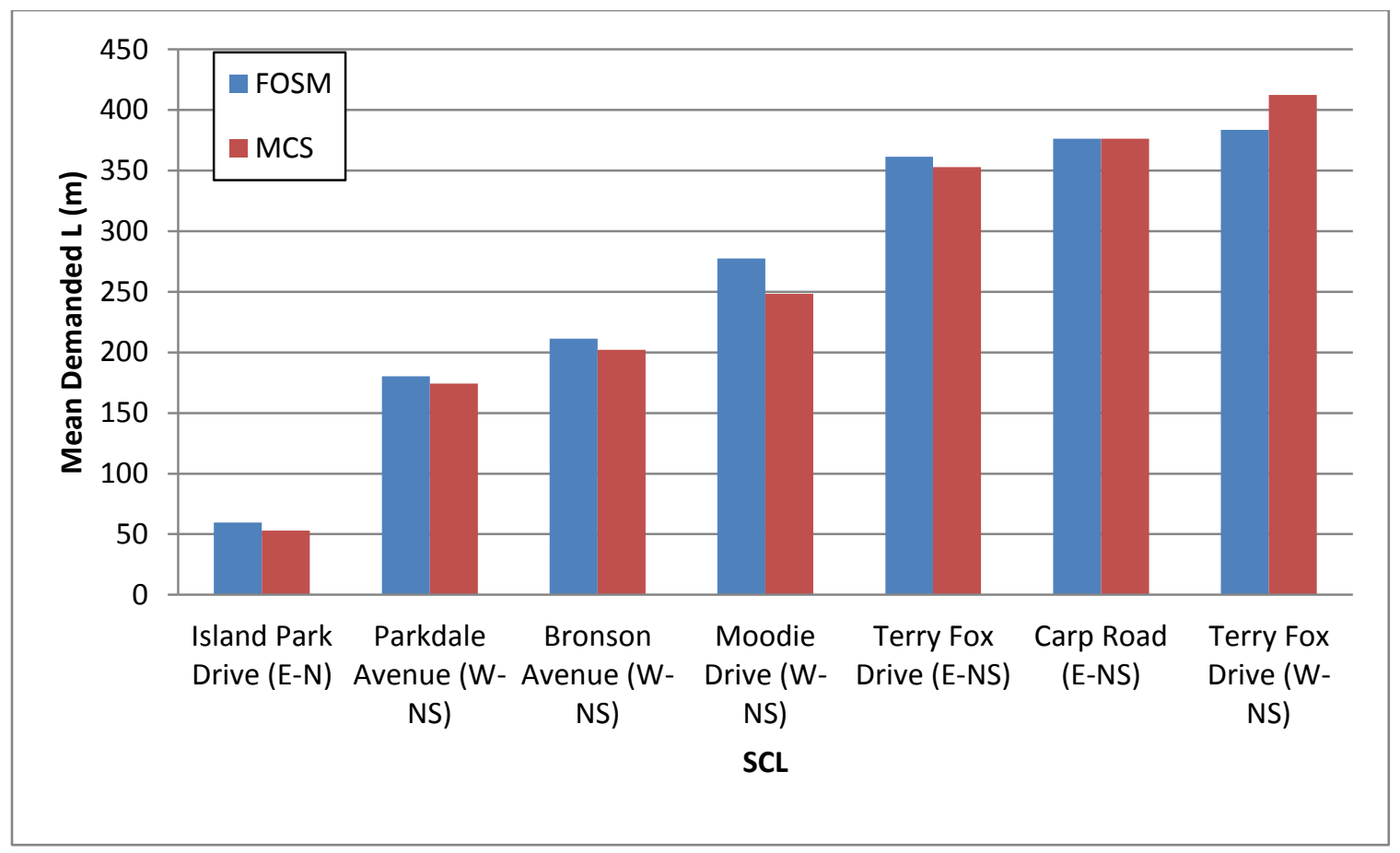

Figure 6.1: Mean Demand L Using FOSM and MCS using the Dataset of each site.

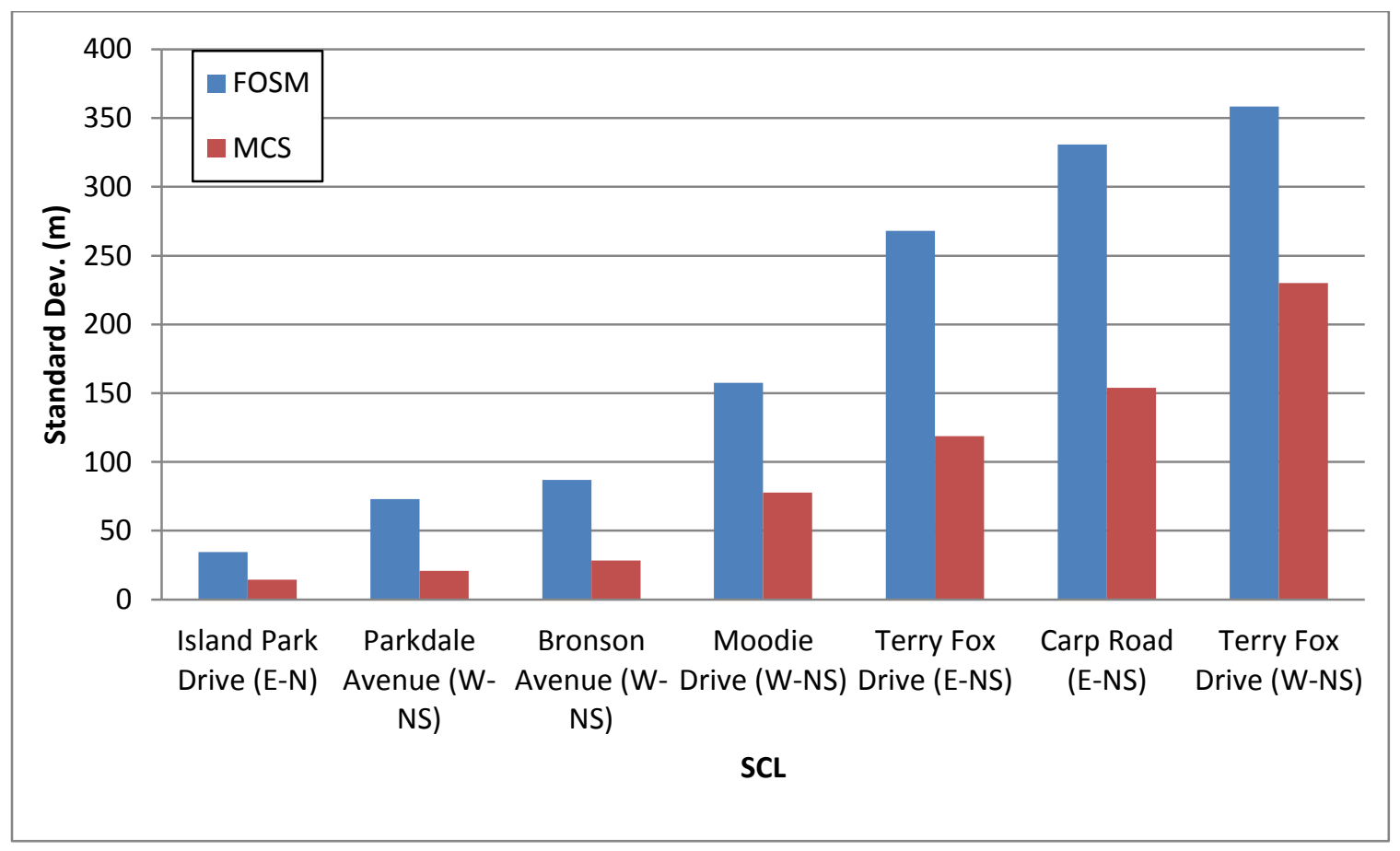

Figure 6.2: Standard Deviation of Demand L Using FOSM and MCS using the Dataset of each site. 


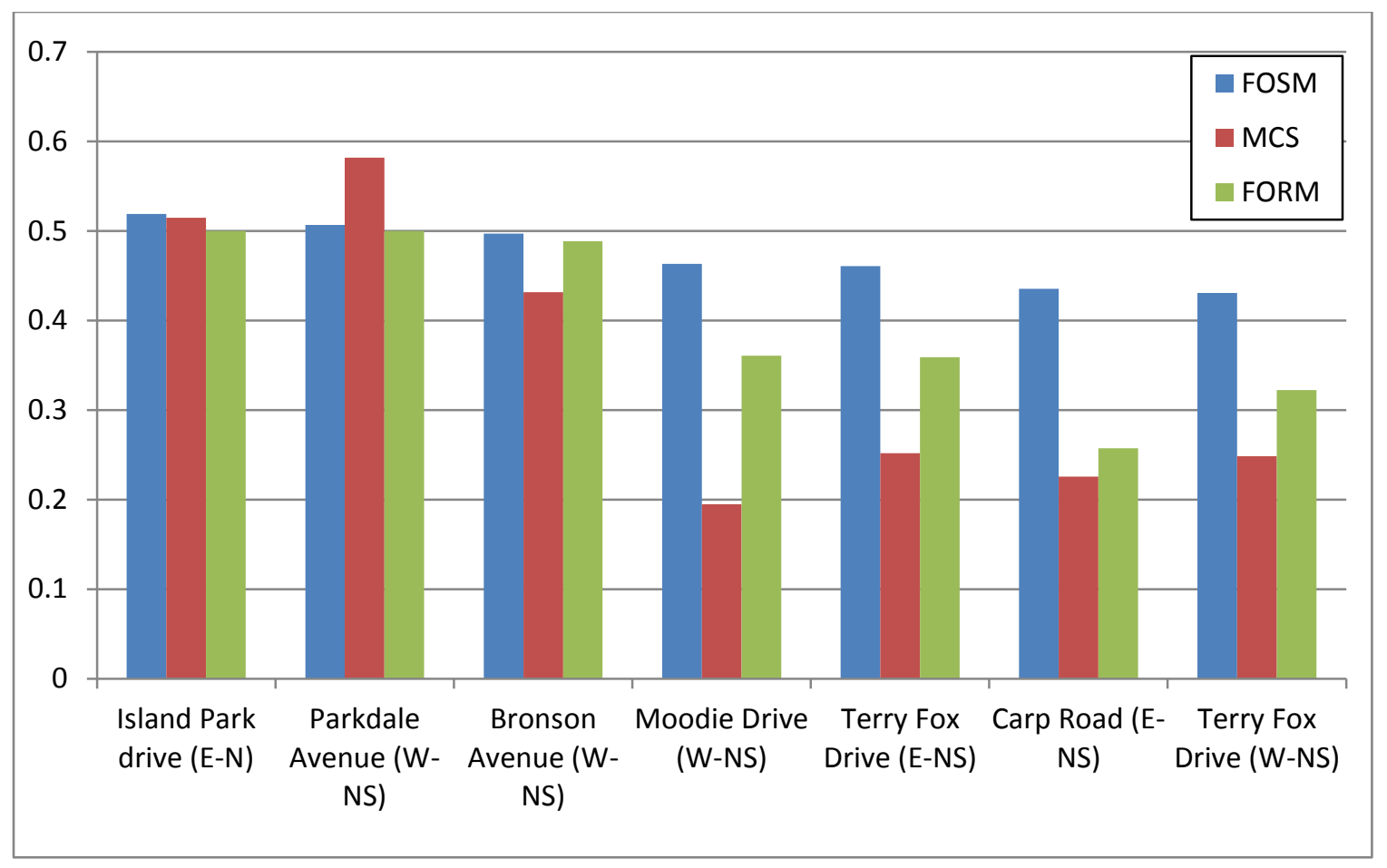

Figure 6.3: PNC Using the Dataset of Each Site (FOSM, MCS, and FORM).

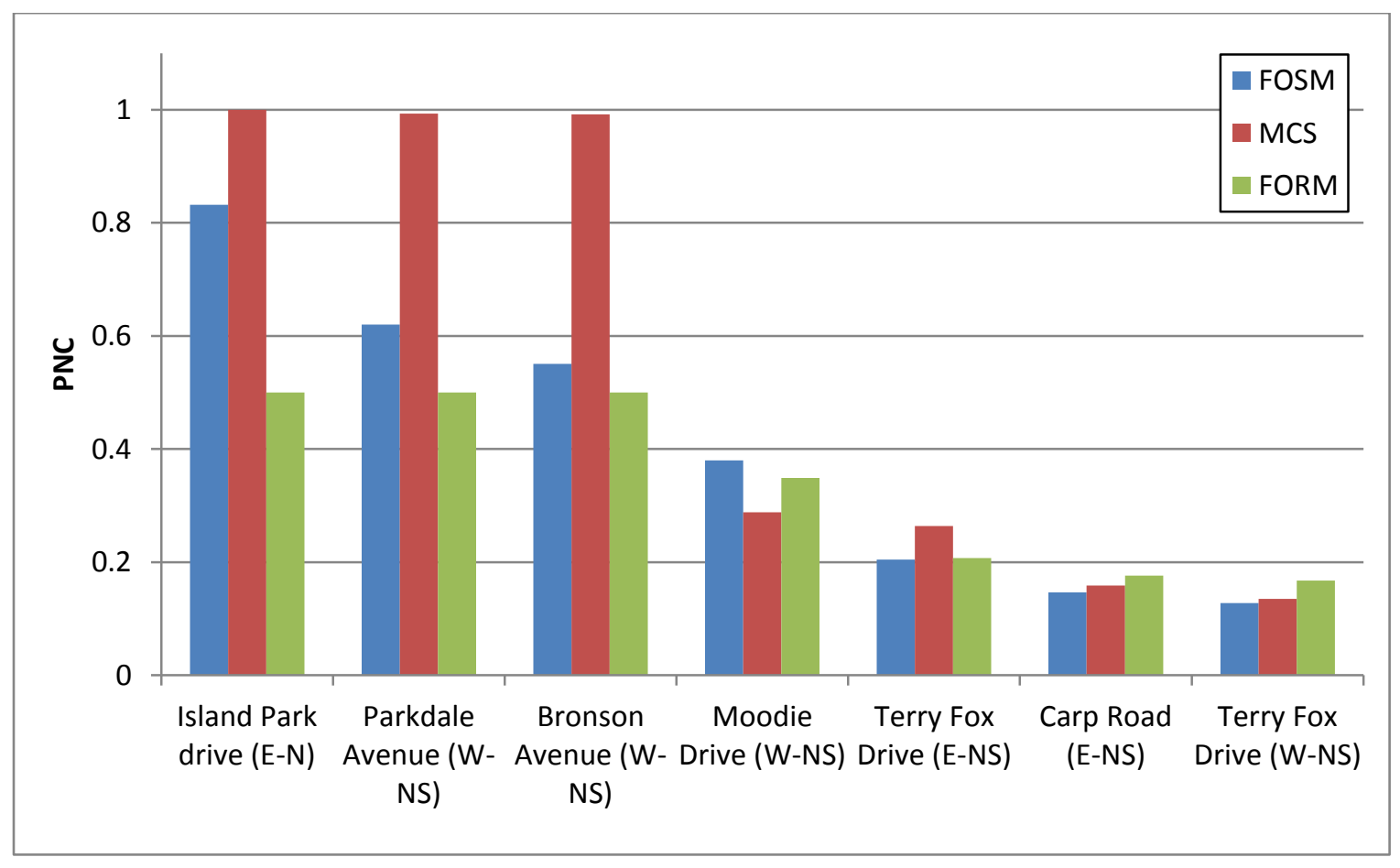

Figure 6.4: PNC Using the Dataset of All Sites Combined (FOSM, MCS, and FORM). 
FORM results suffer from having a maximum PNC of 0.5 due to the physical interpretation of the used methodology, mentioned in Section 5.5. Therefore, the developed FORM model should be applied carefully to sites with short SCL length. As mentioned in Section 6.1, FOSM model using the dataset of each site tends to overestimate $\mathrm{PNC}$ on sites with long $\mathrm{SCL}$ (where $\mathrm{PNC}<0.5$ ). This conclusion was validated when the results were compared to MCS. This can be attributed mainly to the model's nonlinearity as it results in high standard deviations. As the model's nonlinearity increases, approximating the standard deviations using a closed form approximation (gradients) reduces the accuracy and results in higher variance. Higher standard deviation in FOSM would reduce the absolute value of the z-score (Equation 5.10). Therefore, FOSM is more appropriate to estimate mean demand SCL length on a specific site, but the PNC results should be interpreted with caution.

The PNC results of the different sites using each site's data are relatively close to each other, while the same results using the data of all sites combined show noticeable differences for each of the three methods used. The close PNC results in Figure 6.3 can be explained by the fact that the data of each site include drivers' adjusted behavior to account for the SCL length. As shown in Table 4.1, as the SCL length decreases, the mean initial speed decreases and mean deceleration rate increases. Such adjustment causes fewer drivers on the shorter SCL to fall within the models' failure zone. On the other hand, the higher mean initial speed and lower mean deceleration rate on longer SCL cause more drivers to fall within the models' failure zone. 


\subsection{Relationship to Safety}

Estimated PNC values and collision data on the study sites were examined for an indication of possible relationship. The trend of change in collision frequency with PNC values was examined using collision data on the through lanes and SCLs in a five-year period (1998-2002). It should be noted that the number of sites in this study does not allow a proper statistical analysis of the relationship between collision frequency and PNC. Rather, the results presented here may only serve as a preliminary evidence of a potential relationship between PNC and collision frequency on exit ramp terminals.

The following figures show the relationship between the total collision frequency (on both through lanes and SCL) as well as collision frequency on the SCL only and the PNC results using the dataset of each site and the dataset of all sites combined, respectively. Figures 6.5 to 6.10 show the relationship between PNC using the dataset of each site and collision frequencies, while Figures 6.11 to 6.16 show the relation between PNC and collision frequencies using the dataset of all sites combined. The figures also show that there is a linear trend line for each plot and shows the corresponding coefficient of determination $\left(R^{2}\right)$. It should be highlighted that collision data should not be modeled using linear regression, and these trend lines are only for illustration of the possible relationship only. As shown in the figures, there is an indication of a relationship between safety performance on the SCL as well as the whole segment (through lanes and SCL) and the PNC of SCLs. As PNC increases, safety performance deteriorates as indicated by higher collision frequencies. 


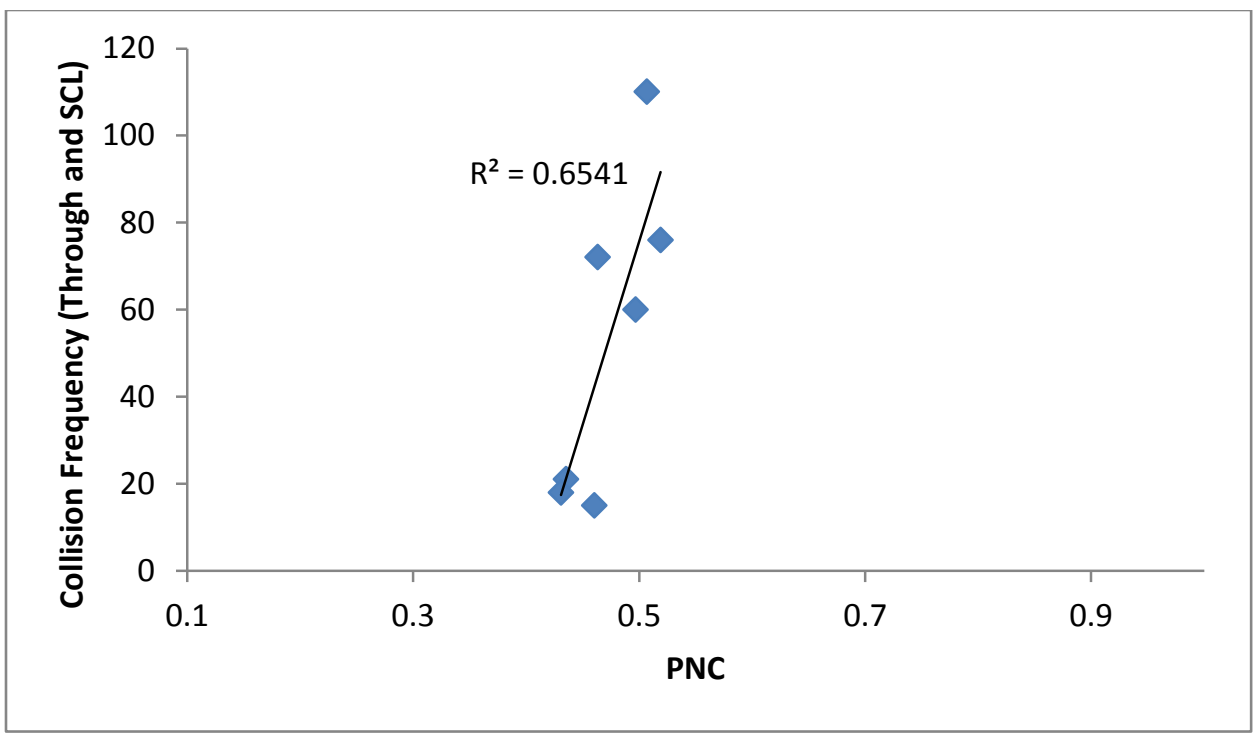

Figure 6.5: FOSM PNC Results Using the Dataset of Each Site and Total Collisions Frequency (Through and SCL)

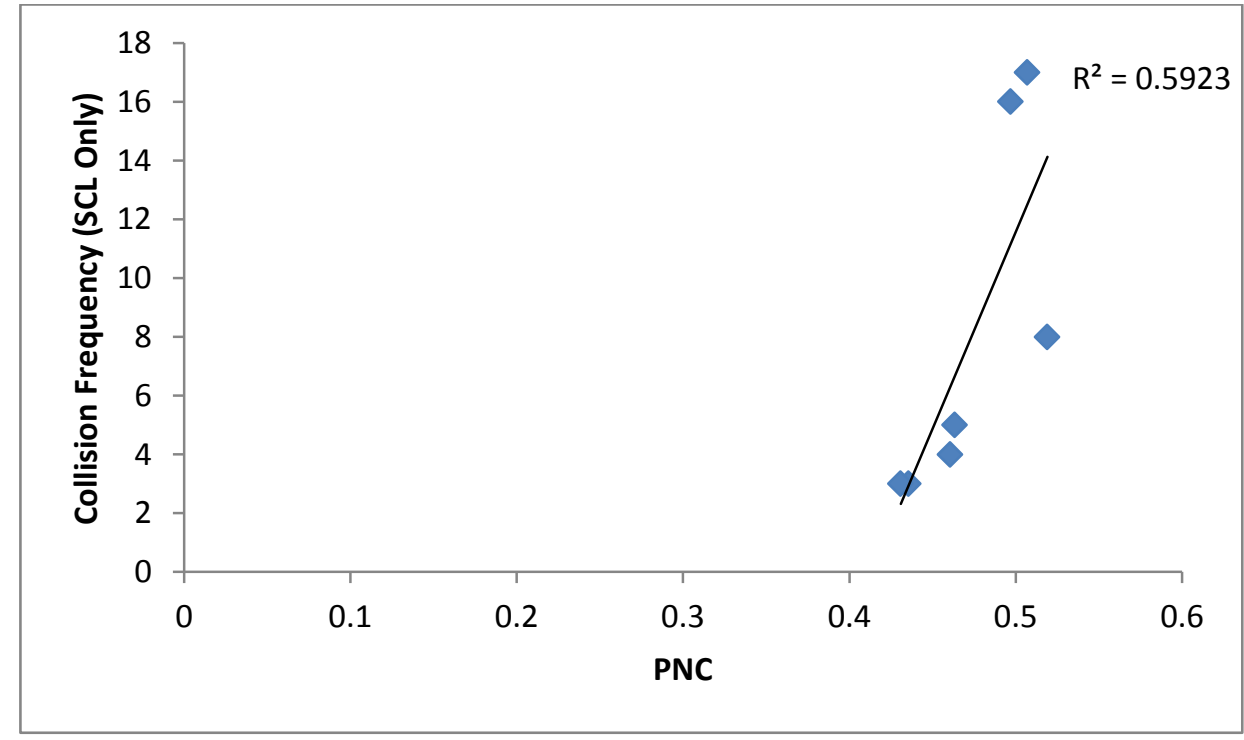

Figure 6.6: FOSM PNC Results Using the Dataset of Each Site and SCL Collisions Frequency 


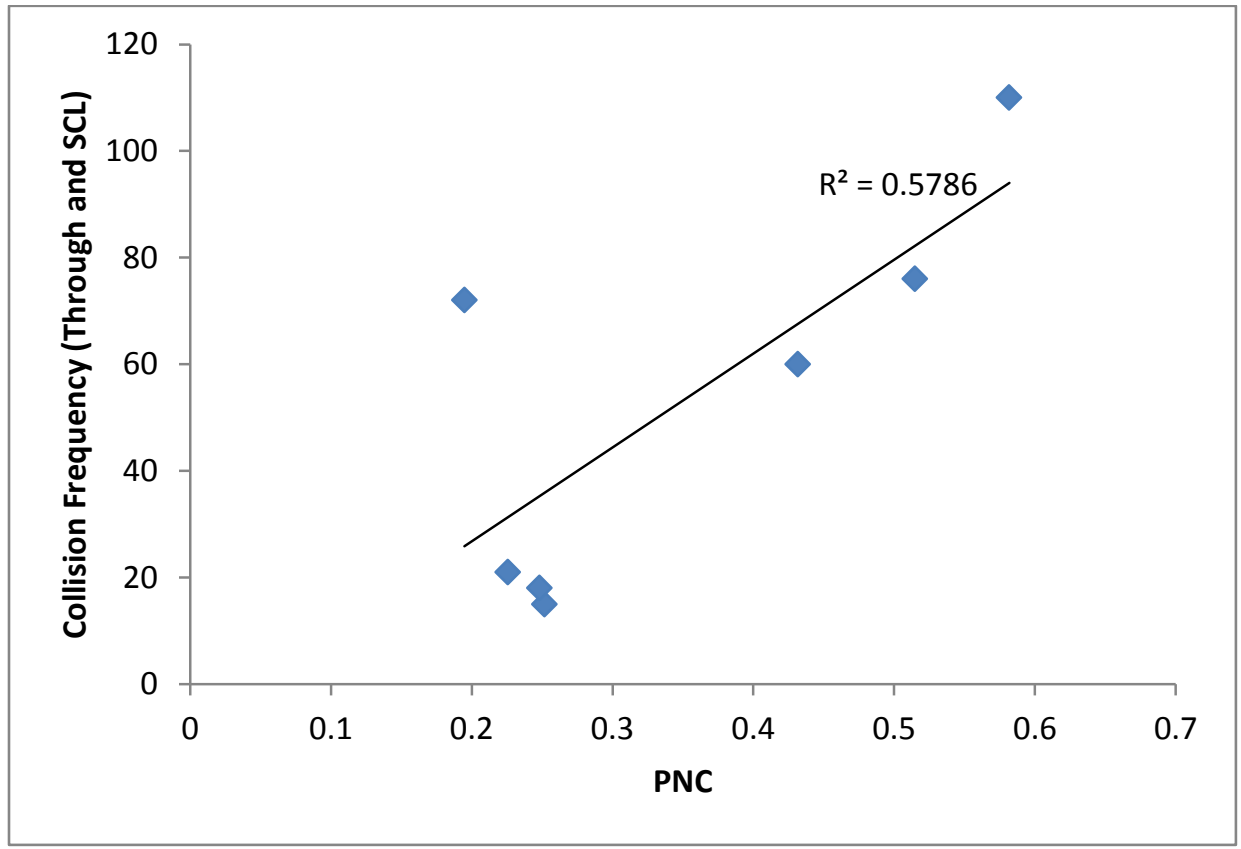

Figure 6.7: MCS PNC Results Using the Dataset of Each Site and Total Collisions

Frequency (Through and SCL)

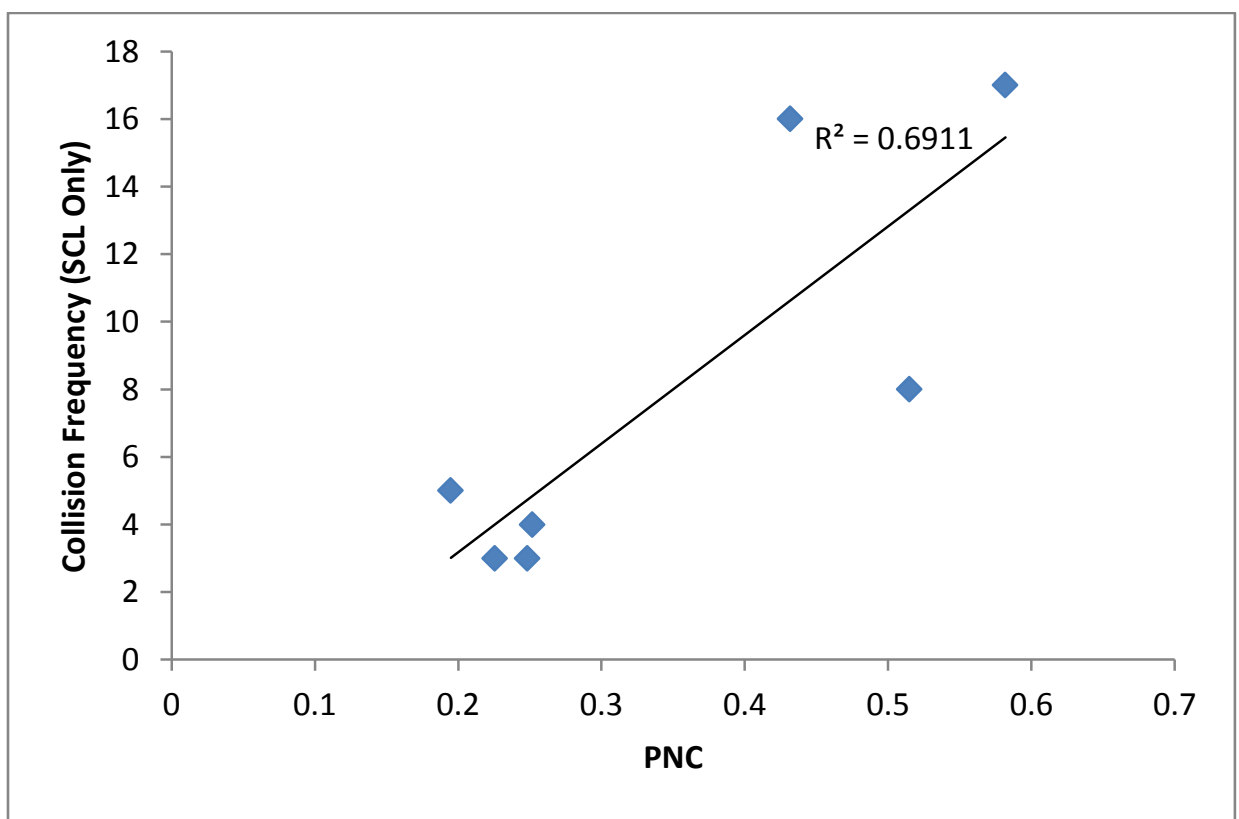

Figure 6.8: MCS PNC Results Using the Dataset of Each Site and SCL Collisions

Frequency 


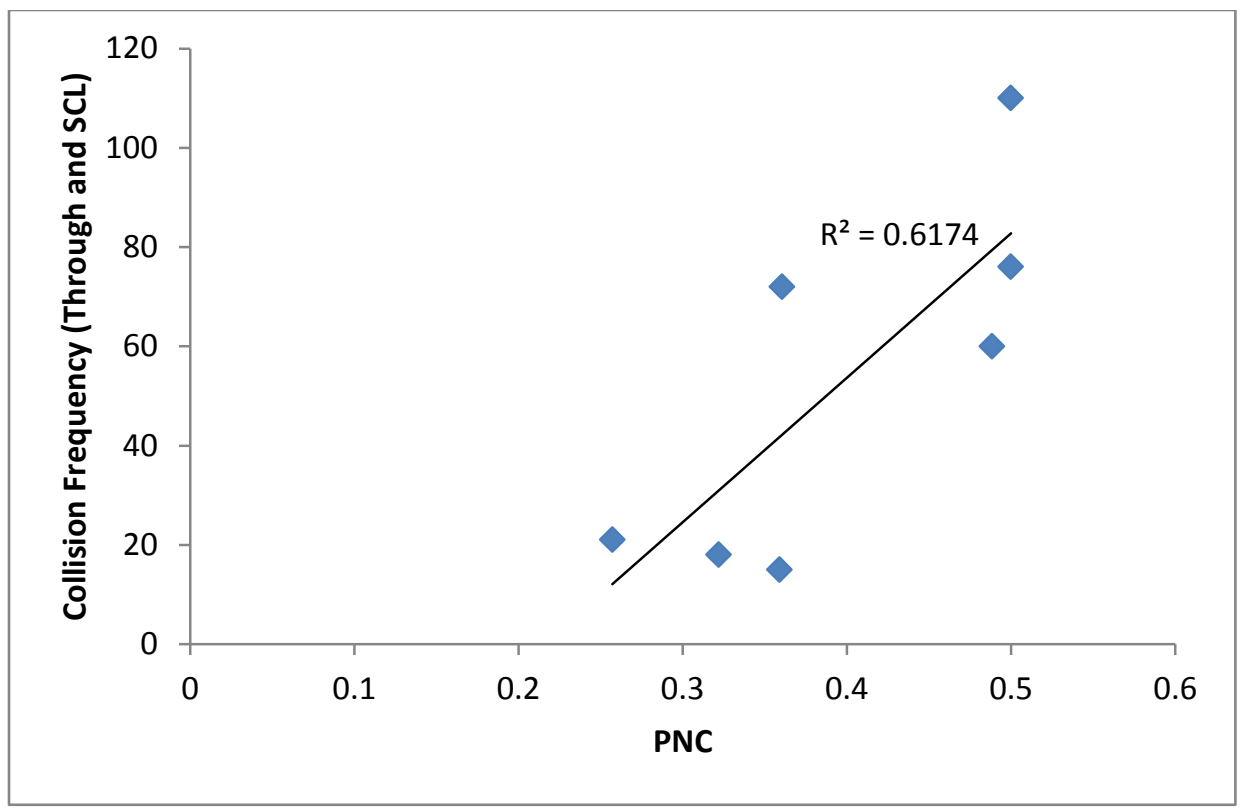

Figure 6.9: FORM PNC Results Using the Dataset of Each Site and Total Collisions Frequency (Through and SCL)

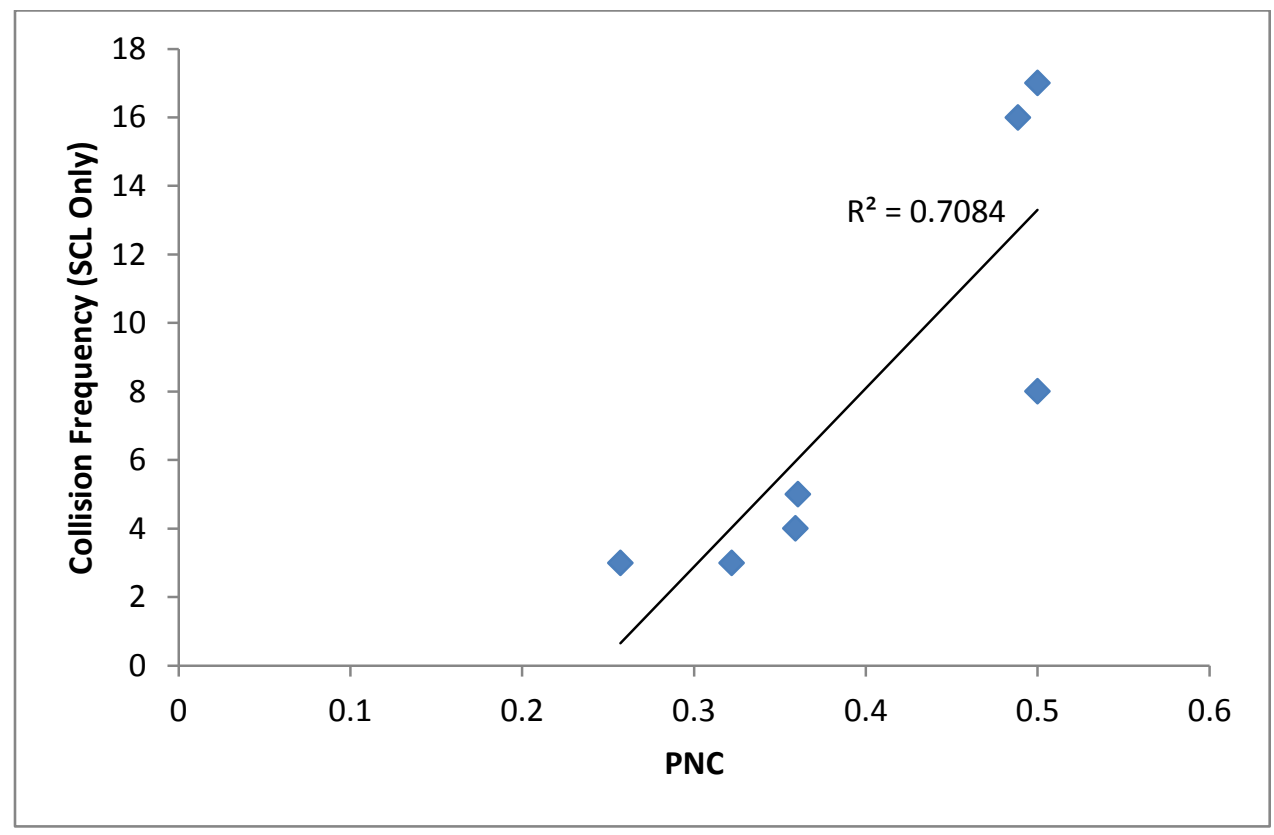

Figure 6.10: FORM PNC Results Using the Dataset of Each Site and SCL Collisions Frequency 


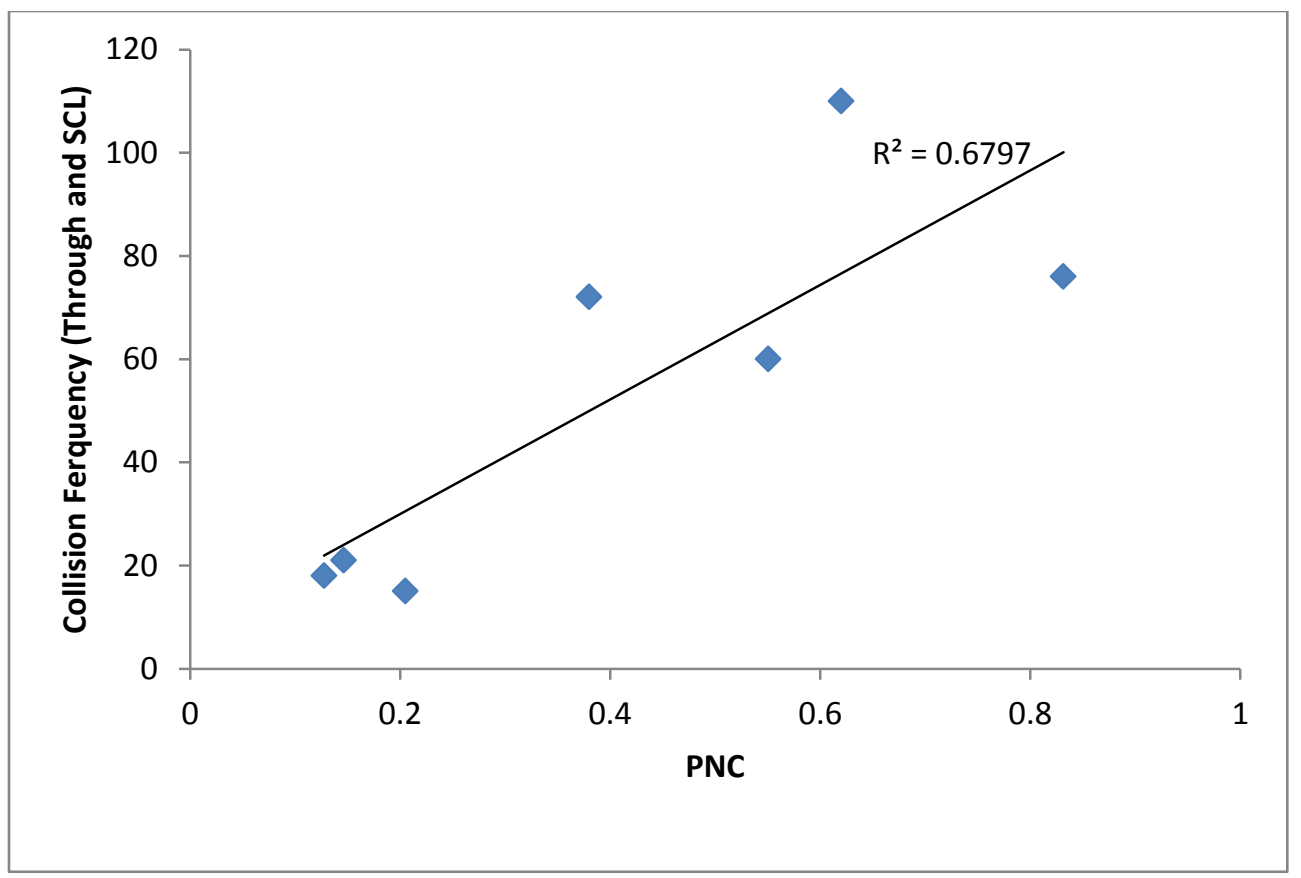

Figure 6.11: FOSM PNC Results Using the Dataset of All Sites Combined and Total Collisions Frequency (Through and SCL)

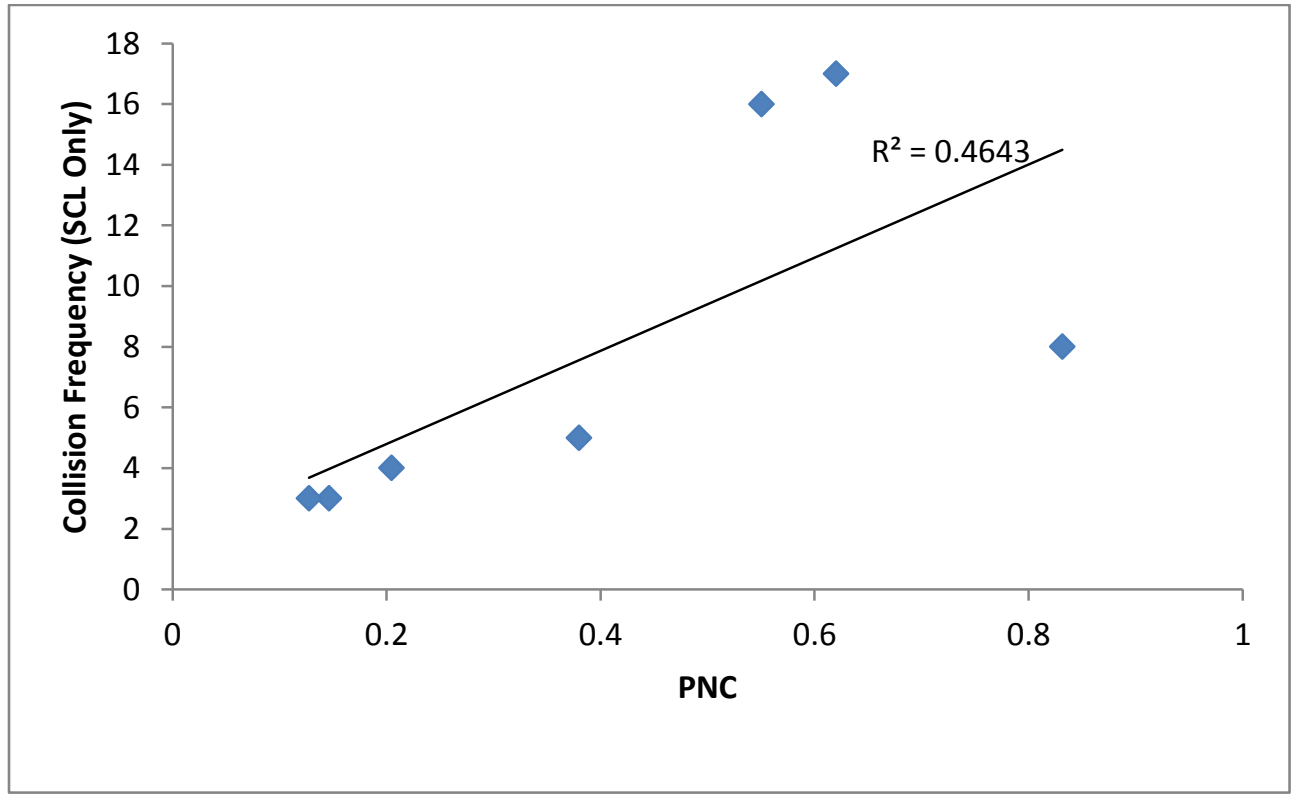

Figure 6.12: FOSM PNC Results Using the Dataset of All Sites Combined and SCL Collisions Frequency 


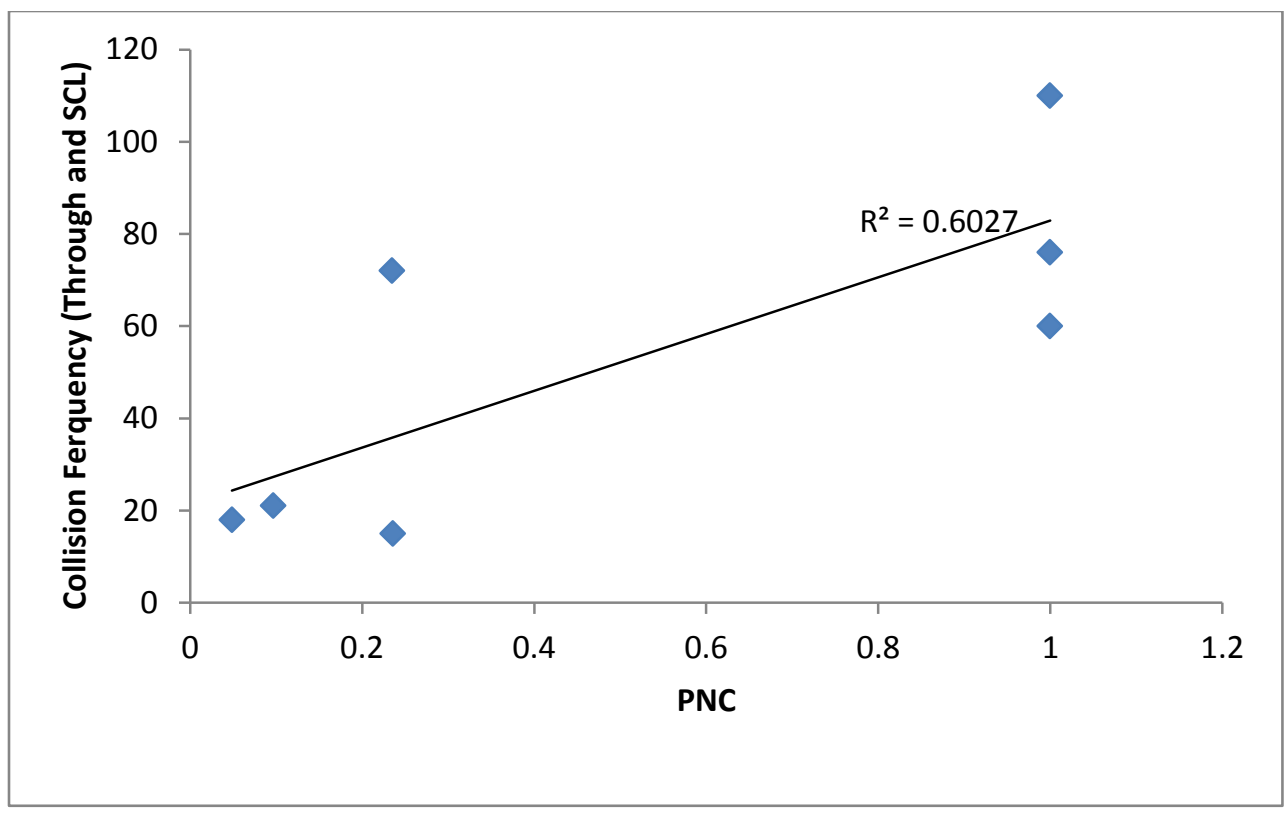

Figure 6.13: MCS PNC Results Using the Dataset of All Sites Combined and Total Collisions Frequency (Through and SCL)

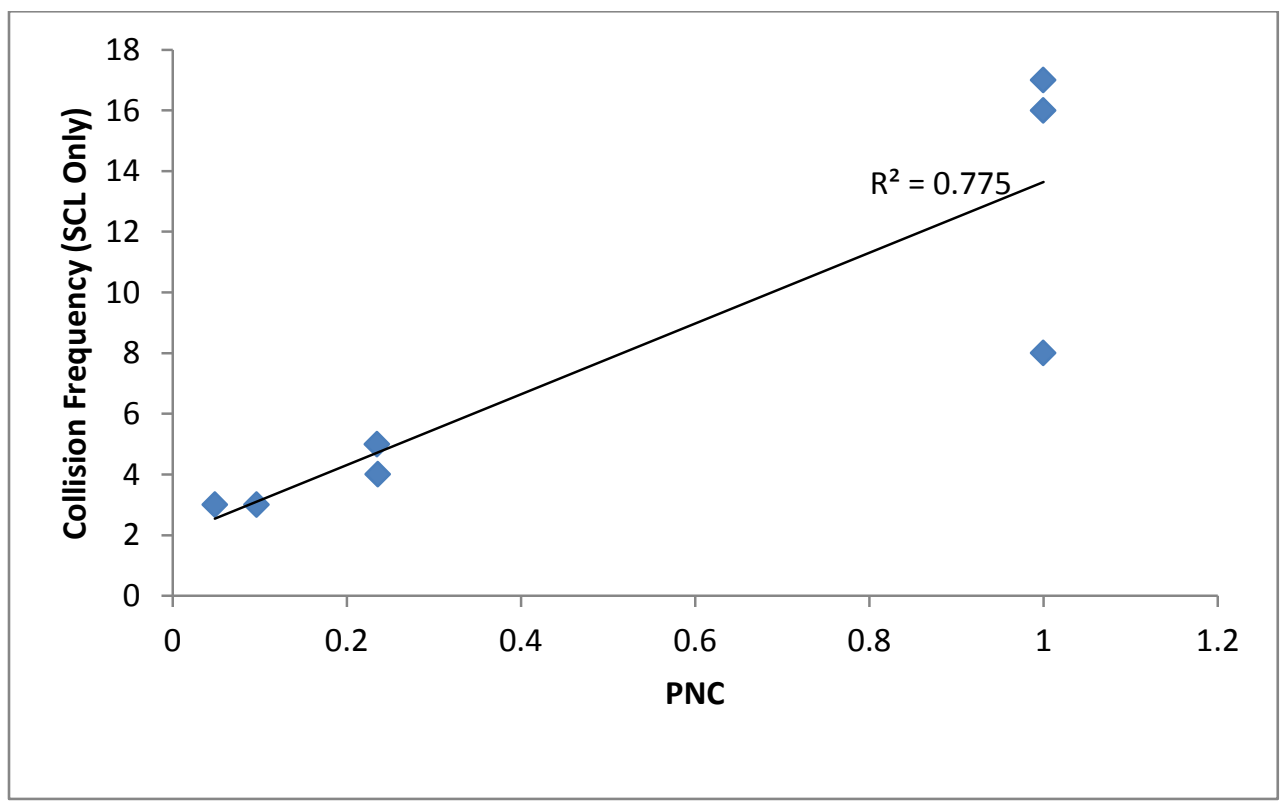

Figure 6.14: MCS PNC Results Using the Dataset of All Sites Combined and SCL Collisions Frequency 


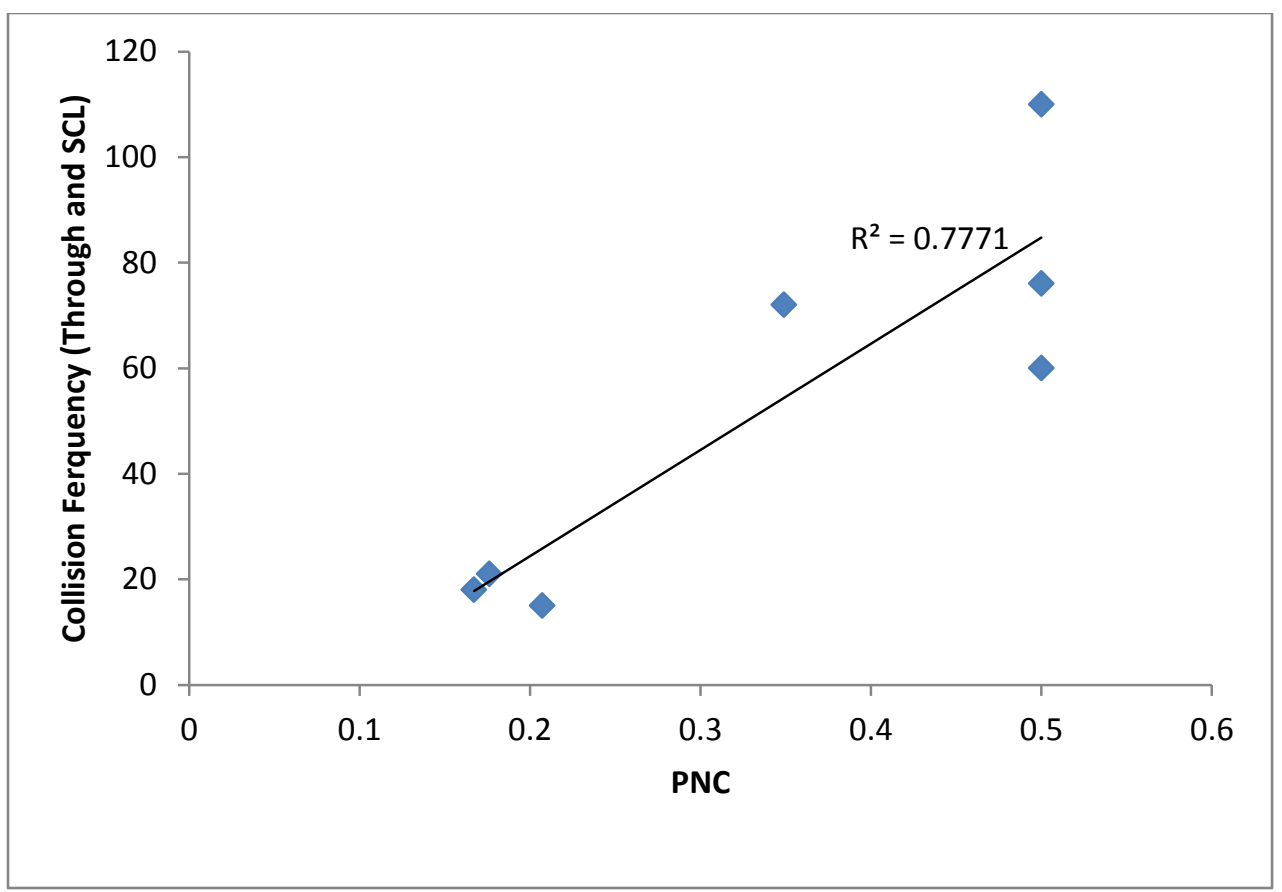

Figure 6.15: FORM PNC Results Using the Dataset of All Sites Combined and Total Collisions Frequency (Through and SCL)

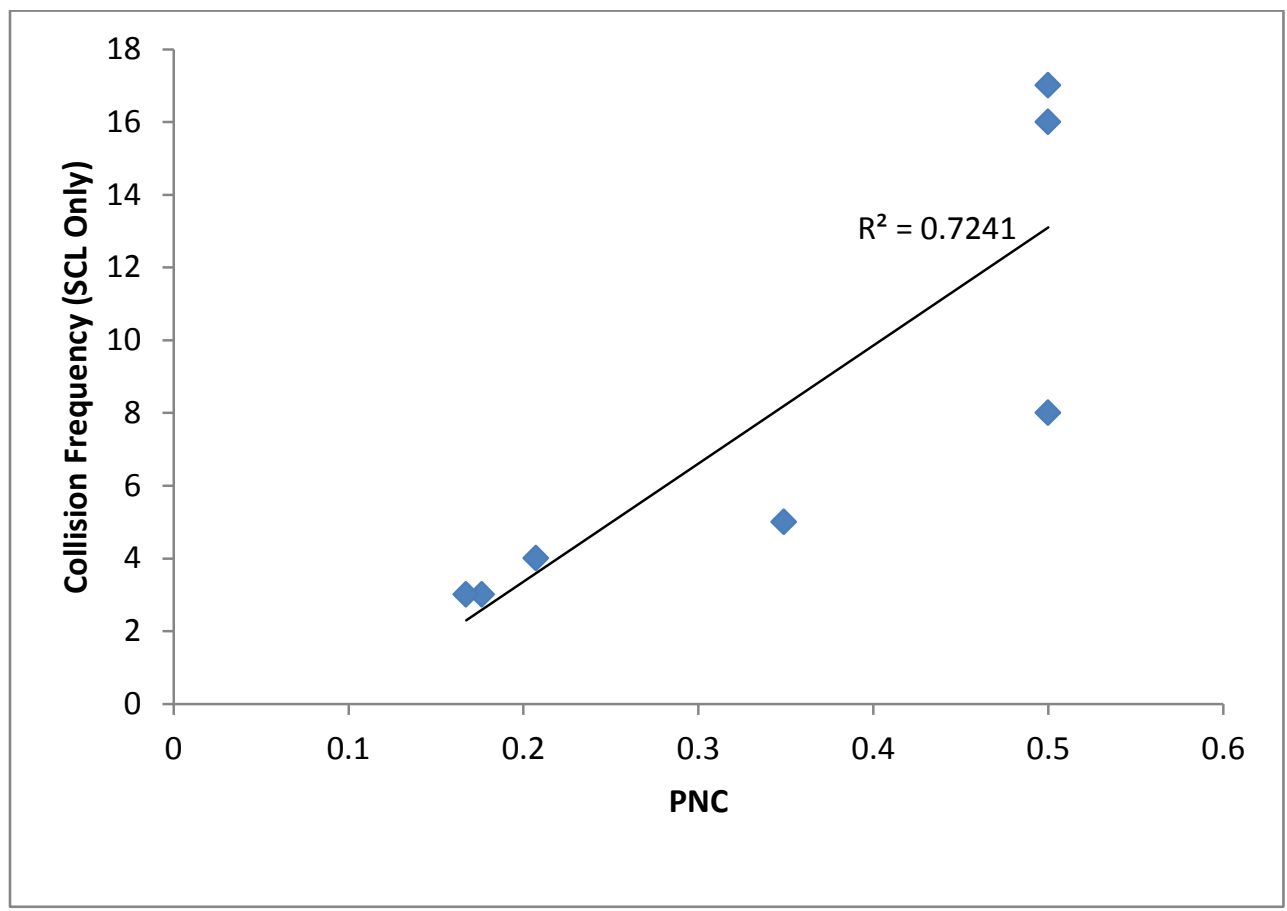

Figure 6.16: FORM PNC Results Using the Dataset of All Sites Combined and SCL Collisions Frequency 


\section{Chapter Seven}

\subsection{Design Application}

This chapter covers a methodology to design the length of limited type SCLs using the reliability method, MCS. Given an estimate of the $85^{\text {th }}$ percentile gore speed and a trial length, the designer should be able to estimate the PNC. Design curves for different combinations of final speeds and SCL lengths were developed and could be used for both evaluation and design. Section 7.1 will cover the initial assumptions and regression models developed to estimate the design parameters. Section 7.2 will cover the design procedure using MCS. The bases of selecting MCS for the design application are the accuracy and its incorporation of the length in predicting the drivers deceleration rates based on the methodology for the dataset of all sites combined (avoiding the issue of FRL deceleration) mentioned in Section 5.4. Based on the initial and final speed, and utilization rate values at different supplied length, deceleration rate values used by drivers were predicted. Previous PNC evaluation (Section 6.2) shows that error of this methodology is very small.

\subsection{Initial Assumptions and Regression Models}

Generally, the information available at the time of designing SCLs is trial design lengths and assumed final speed at the end of the SCL or the gore area. To be able to design limited-length SCLs based on PNC, regression models were developed to predict all parameters distributions based on the length and $85^{\text {th }}$ percentile gore speed. The developed regression models are based on the means of each parameter on the seven 
study sites. These were then used as a distribution for the random generated correlated parameters. Then, deceleration rates were predicted based on these independent correlated parameters. Finally, demand length and PNC were calculated and design curves were developed to estimate PNC based on the selected trial lengths and $85^{\text {th }}$ percentile gore speed. A designer would have to estimate PNC based on only two key parameters, which are $85^{\text {th }}$ percentile gore speed and trial length. Initially, the data known for each SCL are (Table 4.1):

- Mean initial and final speeds, utilization and deceleration rates.

- Standard deviation of initial and final speeds, utilization and deceleration rates.

- Available SCL length.

As explained in chapter 6.2 for MCS using the dataset of all sites combined, the ANOVA test conducted in Section 4.2.2 indicates that deceleration occurs in the FRL. Therefore, the distribution (mean and standard deviation) of initial speed at long SCLs was used for the design application purpose. Mean final speed was modeled using multiple linear regression and was found to depend only on the $85^{\text {th }}$ percentile final speed and its standard deviation. The model has a high $R^{2}$ value as shown equation 7.1 (regression output is in Appendix 8).

$$
v_{f}=1.762+0.967 v_{f, 85^{t h}}-1.310 * \sigma_{v_{f}}, \quad R^{2}=0.997 \text { (Equation 7.1) }
$$

Utilization rate was found to have no relation with any of the variables. Therefore, mean utilization rate of dataset of all sites combined was used to represent all sites. Standard deviations of utilization rate, final speed were not found to have any significant 
relationship with the other variables. Standard deviation of the initial speed depends on only the $85^{\text {th }}$ percentile final speed with $R^{2}$ value equal to 0.618 (Equation 7.2). Since only one parameter (standard deviation of initial speed) was found to have a significant relationship, standard deviations of the dataset of all sites combined were used as standard deviations for all variables $\left(v_{i}, v_{f}\right.$, and, $\left.U\right)$. Complete regression output could be found in Appendix 8.
$\operatorname{Std}\left(v_{i}\right)=1.205+0.0 .047 v_{f, 85^{t h}}$
$R^{2}=0.618$
(Equation 7.2)

\subsection{Design Application Using MCS}

MCS was used to generate deign curves using the same methodology discussed in Section 5.4. The only difference is that the distribution of final speed was based on the $85^{\text {th }}$ percentile gore speed modeled using the study sites in section 7.1, while the standard deviation of final speed was taken as the standard deviation of the dataset of all sites combined. Following is a summary of the steps used.

1. Eighty fifth percentile gore speed is a trial input by the designer.

2. Trial design length is selected as another input by the designer.

3. Distribution of initial speed is taken as distribution of initial speed at long SCLs where, $\mu_{v i \_l o n g}=29.085 \mathrm{~m} / \mathrm{s}(107.705 \mathrm{~km} / \mathrm{h})$ and $\sigma_{v i \text { long }}=2.472 \mathrm{~m} / \mathrm{s}(8.899 \mathrm{~km} / \mathrm{h})$. Similar to that defined in Section 4.2.2.

4. Mean final speed is found using Equation 7.1.

5. Mean utilization rate is the mean of all sites combined dataset's utilization rate (Table 4.1). 
6. Standard deviation final speeds and utilization rate were taken as the standard deviation of the dataset all sites combined (Table 4.1).

7. Then, the same procedure described in Section 5.4 is followed starting with:

a. Random numbers were generated and correlated using Cholesky's decomposition of the correlation matrix (correlation coefficients are shown in Table 4.1).

b. Correlated numbers were then converted to follow the distributions of the means and standard deviations found in steps 3 to 6 using the inverse of the normal cumulative distribution

c. Deceleration rate was predicted using Equation 5.11 for the dataset of all sites combined.

d. The screening steps mentioned in chapter 5.4 were performed and demand length was calculated.

e. PNC was calculated for the selected trial length and $85^{\text {th }}$ percentile gore speed.

Based on the data's range, a range between $23 \mathrm{~m} / \mathrm{s}$ and $32 \mathrm{~m} / \mathrm{s}(83 \mathrm{~km} / \mathrm{h}$ and 115 $\mathrm{km} / \mathrm{h}$ ) was used as the $85^{\text {th }}$ percentile final speed. Similarly, supply lengths $(S L)$ between $60 \mathrm{~m}$ and 400 were used to generate the design curves. MCS design Script could be found in Appendix 11. Figure 7.1 shows a summary of the developed curves. 


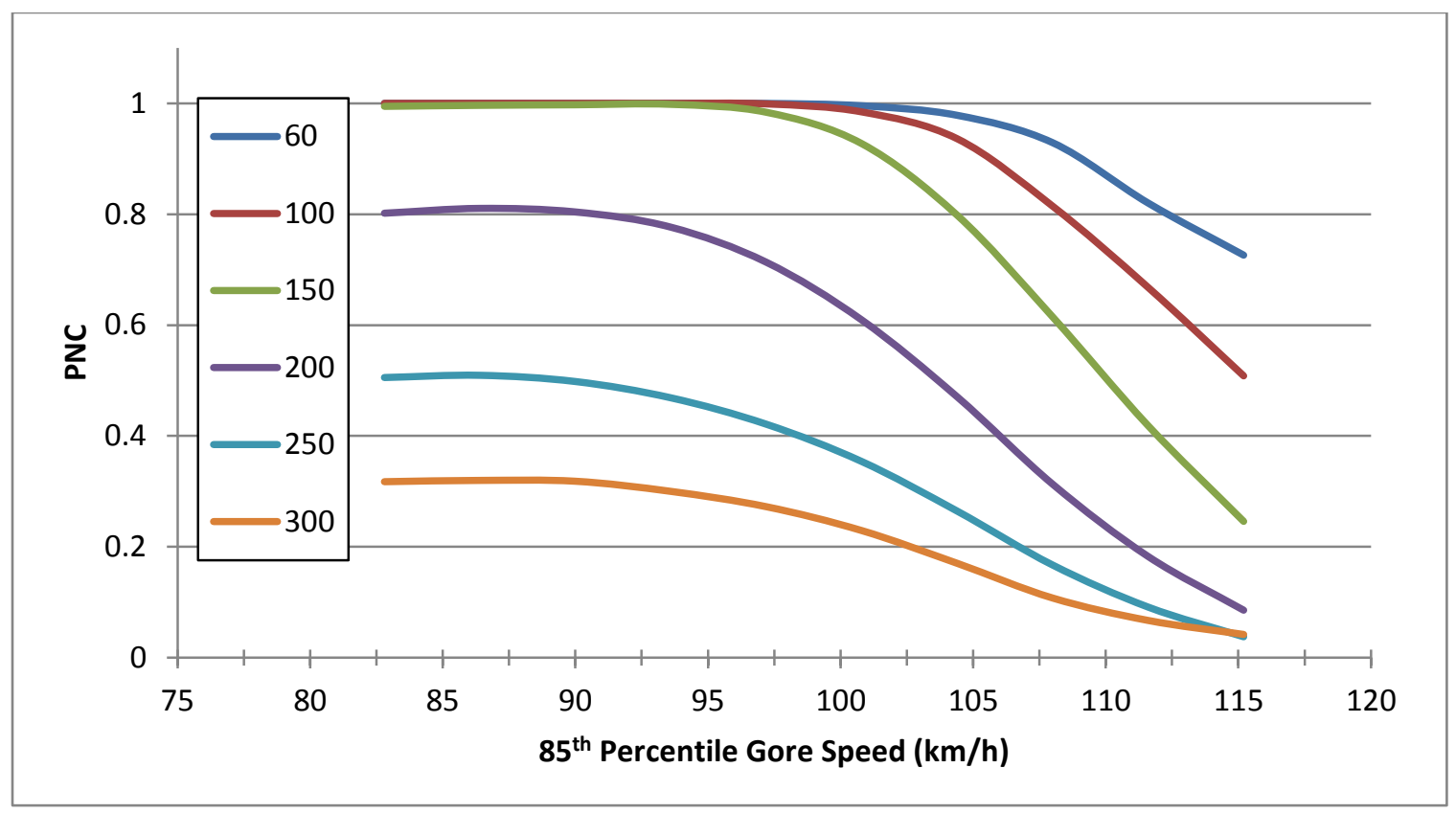

Figure 7.1: Design Curves for Limited SCLs Based on 85th Percentile Final/Gore Speed and PNC for Lengths $\leq 300 \mathrm{~m}$.

It was found that the design curves work for only for lengths less than $300 \mathrm{~m}$. As can be noticed in Figure 7.1, the $300 \mathrm{~m}$ curve PNC value exceeds the $250 \mathrm{~m}$ curve at an 85 th percentile gore speed of $115 \mathrm{~km} / \mathrm{h}$. When the distribution testing was performed to compare the distribution of the generated numbers to the original data, it was found that, for long SCLs (exceeding $300 \mathrm{~m}$ ) the distribution of utilization and deceleration rates shows lower means and standard deviation than the original dataset values. It could be possible that more data representing a wider range of SCL lengths are further needed and the current database is not enough to represent the distribution of drivers or to design for long SCLs. Figure 7.2 shows the design application using MCS for an SCL length of 350 $\mathrm{m}$ in addition to the previous lengths. 


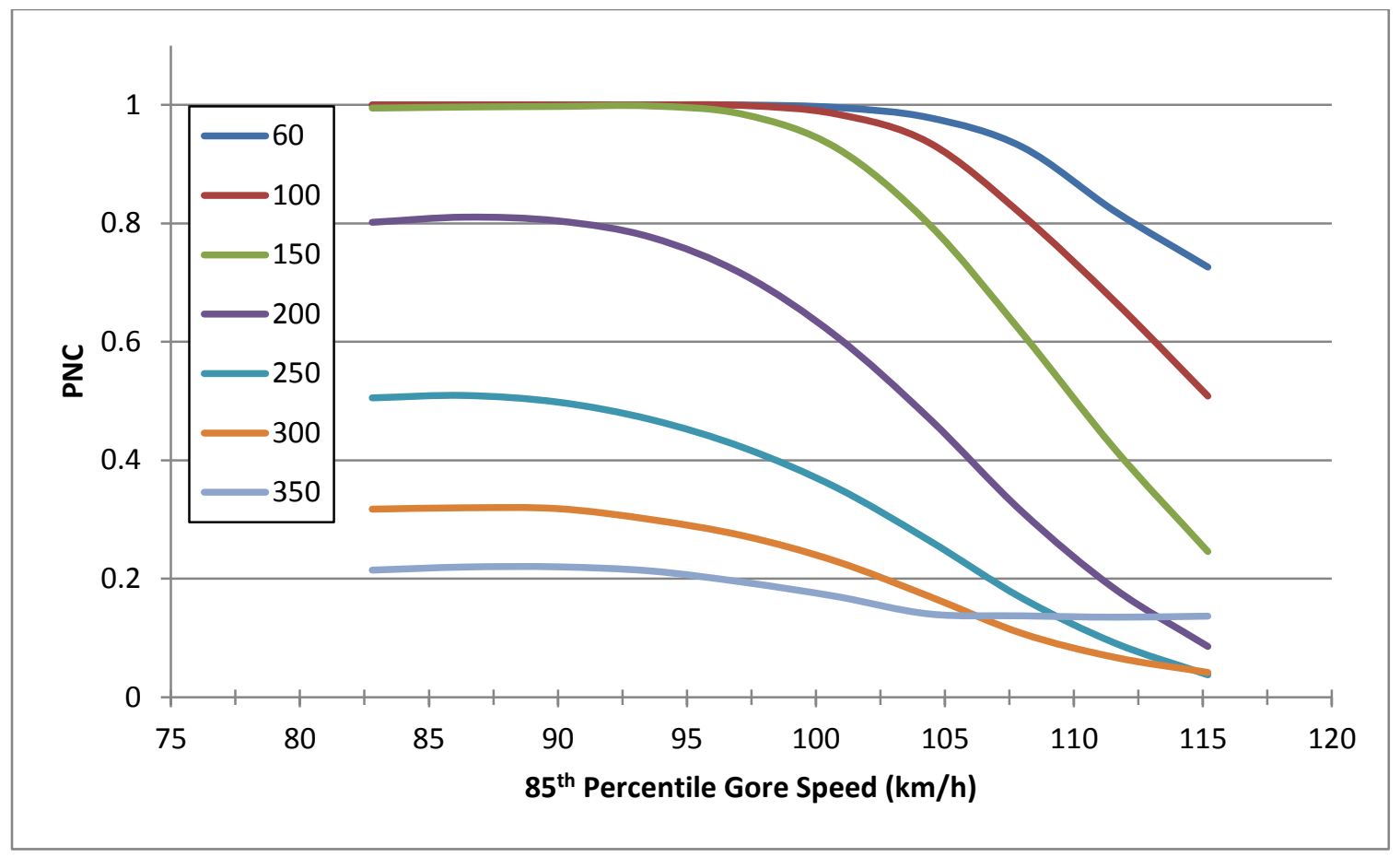

Figure 7.2: Design Curves for Limited SCLs Based on 85th Percentile Final/Gore Speed and PNC Including $L>300$.

As can be seen in Figure 7.2 , at an $85^{\text {th }}$ percentile gore speed of $106 \mathrm{~km} / \mathrm{h}$, the PNC value for $350 \mathrm{~m}$ is the same PNC for the $300 \mathrm{~m}$ SCL. Afterwards, PNC values on the $350 \mathrm{~m}$ curve exceed PNC values on the $300 \mathrm{~m}$ curve. It was expected that the PNCs for $350 \mathrm{~m} \mathrm{SCL}$ curve at all points to be lower than $300 \mathrm{~m} \mathrm{SCL}$ curve, which is not the case. This could be possibly due to the reason discussed earlier. 


\section{Chapter Eight}

\subsection{Conclusions and Recommendations}

\subsection{Conclusions}

Efficient operation of freeway Speed Change Lanes (SCLs) reflects the design capability in accommodating decelerating vehicles on the SCL. In this study, probability of noncompliance (PNC) (demand exceeding supply) between the demand deceleration length by the road users and available supply SCL length was found at seven sites. The seven sites of this study were limited type SCLs from Highway 417 in the City of Ottawa, Ontario. Speed data were taken from El-Basha (2006) and this study is a continuation to his driver behaviour modeling efforts. Speed profiles on SCLs and FRL were used to evaluate each site and draw conclusions about the operational performance on the selected study sites. Three methods were used to evaluate PNC for the study sites: FOSM, MCS, and FORM. The models were developed assuming that drivers do not utilize the full SCL available length. Rather, drivers diverge off the FRL onto the SCL and maintain, accelerate, or decelerate slightly for some distance on the SCL if it is judged to be too long. After that, it was assumed that drivers start the deceleration some distance from the gore area and adopt a constant deceleration rate.

FOSM was found to give a good estimation of the mean demand deceleration length; however, it overestimates the standard deviation compared to the simulation approach (MCS). Due to the high standard deviation, PNC values are either an overestimation at longer sites or an underestimation at shorter sites. MCS is an accurate technique that simulates each driver based on the data's distribution. When the evaluation 
was carried out for each site using the dataset of all sites combined in MCS, mean initial speed at long SCLs was used as an initial speed to avoid the possible condition of drivers decelerating at the FRL. Mean final speed at each site, mean utilization rate of the dataset of all sites combined, and parameters' standard deviations of the dataset of all sites combined were used to represent the distribution of the variables at the study sites. FROM, which is based on a truncated Taylor series, is a more accurate procedure than FOSM. However, the ellipsoid approach used has the limitation of a maximum PNC value of 0.5 . Therefore, PNCs generated for short lanes should be interpreted carefully.

The trend of change in collision frequency with PNC values was examined using collision data on the through lanes and SCLs in a five-year period (1998-2002). It should be noted that this was not a collision modeling attempt because of the small number of sites available in this study. It was evident that there is an indication of a relationship between PNC and safety on the selected study sites. It was found that there is a trend of decreasing collision frequency with decreasing PNC. Many studies on safety of deceleration SCL found that collision frequency decreases as the SCL length increases as discussed in Section 2.3. However, other studies have reported an opposite trend where collision frequency was found to increase at longer deceleration SCLs (Chen, Liu, Lu \& Behzadi, 2009). A possible reason to such result is that drivers may perform acceleration on the SCL, and then decelerate when they are closer to the gore which could eliminate possible effects of the extra length provided. Another reason is that drivers may perform weaving maneuvers on longer SCLs; drivers may change their lane back from the SCL to the FRL either because drivers got to the SCL by mistake or drivers used the SCL to pass 
a slower vehicle in the FRL. It should be noted that such maneuvers were not observed on the limited type SCLs of this study.

A design application attempt was performed using MCS. The reason behind the choice of MCS is the accuracy of this technique. FOSM and FORM are less accurate approximation techniques as they are first order truncated Taylor series. However, they are accurate enough for many engineering applications (Section 2.4). Designers would be

able to find the PNC of any trial lengths, $85^{\text {th }}$ percentile gore speed, using the generated design curves, which are based on the distribution of mean initial speed of long SCLs of the dataset in this study. Seven design curves for trial design lengths ranging between 60 $\mathrm{m}$ and $300 \mathrm{~m}$ were developed and it was found that the design application is only applicable to SCL lengths less than $300 \mathrm{~m}$. further dataset might be needed to find more relationships between the study parameters. For example, it was found that there was no relationship between utilization rate distribution and the rest of the parameters' distributions. Utilization rates are lower at long sites. It could be possible that more data at longer SCLs should be examined for a possible relationship.

\subsection{Recommendations for Future Studies}

Based on the findings of this study, the following are recommended as future work in the area of evaluating operational performance for SCLs:

- The current approach is a way for designers to examine the impact of their design choices of lengths (for $S L<300 \mathrm{~m}$ ) to the operational performance at site. Given enough collision database, developed models could be used to develop a safetyexplicit design approach to examine the impact of design decisions on safety 
performance. To be able to do so, a statistical relationship between collision frequencies at SCL sites and PNC values needs to be developed.

- For higher accuracy, the same models could be applied using non-constant deceleration rates at long SCLs. The developed models could be expanded to consider deceleration behavior utilizing a low deceleration rate at the beginning of the SCL followed by harder one similar to the methodology of the Green Book discussed in Section 2.1.

- The methodology applied in this study considers only one exit limited type SCLs. It is recommended that the models are expanded to cover dual exit limited-length and extended-length SCLs as well. Special measures should be taken to account for the weaving maneuvers on extended type SCLs. this would help in future assessment of safety on extended SCLs as the same freeway section used in this study was observed to have more collisions on extended SCL compared to limited-length SCL (Sarhan, Hassan, \& Abd El Halim, 2008). 


\section{List of References}

AASHTO. (2011). "A Policy on Geometric Design of Highways and Streets" (6th ed.). American Association of State Highway and Transportation Officials. Washington, D.C.

Abia, S. D. (2010). “Application of Reliability Analysis to Highway Design Problems: Superelevation (e) Design, Left Turn Bay Design-Safety Evaluation and Effect of Variation of Peak Hour Volumes on Intersection Signal Delay Performance". Retrieved July 15, 2012.

Bared, J., Giering, G. L., \& Warren, D. L. (1999). "Safety Evaluation of Acceleration and Deceleration Lane Lengths". Institute of Transportation Engineers , 50-54.

Bauer, K., \& Harwood, D. (1998). "Statistical Models of Accidents on Interchange Ramps and Speed Change Lanes”. Federal Highway Adminstration. Washingtonm D.C.

Bellosta, C. J. (2011, December 28). ADGofTest. Retrieved July 31, 2013, from cran.rproject: http://cran.r-project.org/web/packages/ADGofTest/ADGofTest.pdf

Bellosta, C. J. (2013, August 29). cran.r-project. Retrieved 11 05, 2013, from Package 'ADGofTest': http://cran.r-project.org/web/packages/ADGofTest/ADGofTest.pdf

Bloombertg, L. (2008, August 18). ITE Meetings. Retrieved from Institute of Transportation Engineers: http://www.ite.org/meetings/2008AM/Session\%2011_Loren\%20Bloomberg.pdf 
Canada's National Highway System Condition report.(2009). Government. Council of Ministers Responsible for Transportation and Highway Safety, Ottawa, Ontario, Canada.

Canadian Motor Vehicle Traffic Collision Statistics: 2010. (2012). Government. Transport Canada, Road Safety and Motor Vehicle Regulation, Ottawa, Ontario, Canada.

Chen, H., Zhou, H., \& Lin, P.-S. (2012). "Selecting Optimal Deceleration Lane Lengths at Freeway Diverge Areas Combining Safety and Operational Effects". Transportation Research Board, paper 12-2555.

Chen, H., Liu, P., Lu, J., \& Behzadi, B. (2009). "Evaluating the Safety Impacts of the Number and Arrangement of Lanes on Freeway Exit Ramps". Accident Analysis \& Prevention, 41(3), 543-551.

Chena, H., Zhoub, H., Zhaob, J., \& Hsuc, P. (2011).” Safety performance evaluation of left-side off-ramps at freeway diverge areas”. Accident Analysis and Prevention, $43,605-612$.

Cirillo, J. (1970). "The Relationship of Accidents to Length of Speed-Change Lanes and Weaving Areas on Interstate Highways". Highway Research Record. 312:17-32.

Dettinger, M. \& Wilson, J., 1981. "First Order Analysis of Uncertainty in Numerical Models of Groundwater Flow: Part 1. Mathematical Development". Water Resources Research, 17(1), pp. 149-161. 
El-Basha, R. H. (2006). "Driver Speed Behaviour on Freeway Deceleration SpeedChange Lanes". Department of Civil and Environmental Engineering, Carleton University. Ottawa: Carleton University.

El-Basha, R. H., Hassan, Y., \& Sayed, T. A. (2007). "Modeling Freeway Diverging Behavior on Deceleration Lanes”. Transportation Research Record (2012), 3037.

Faber, M. H. (2007). "Risk and Safety in Civil Engineering”. Swiss Federal Institute of Technology. Zurich.

Fatema, T., \& Hassan, Y. (2013). "Probabilistic Design of Freeway Entrance Speed Change Lanes Considering Acceleration and Gap Acceptance Behavior”. Transportation Research Record, (in press).

Fatema, T., Ismail, K., \& Hassan, Y. (2014). "Validation of a Probabilistic Model for the Design of Freeway Entrance Speed Change Lanes". Transportation Research Board, Paper 2014-3236 (in press).

Federal Highway Adminstration. (2004). "Signalized Intersections: Informational Guide". Federal Highway Adminstration, Producer, \& U.S. Department of Transportation. Retrieved September 03, 2013, from FHWA: http://www.fhwa.dot.gov/publications/research/safety/04091/12.cfm

Felipe, E. L. (1996). "Reliability-Based Design for Highway Horizontal Curves". University of British Colombia. British Columbia. 
Fitzpatrick, K., Chrysler, S. \& Brewer., a. M., 2012. "Deceleration Lengths for Exit Terminals". Journal of Transportation Engineering, 138(6), pp. 768-775.

Harwood, D., Bauer, K., Potts, I., Torbic, D., \& Richard, K. (2002). "Safety Effectiveness of Intersection Left- and Right-Turn Lanes". Federal Highway Adminstration. Retrieved September 2013, from http://www.fhwa.dot.gov/publications/research/safety/02089/index.cfm

Hassan, Y., Sarhan, M., \& Salehi, M. (2012). "Probabilistic Model for Design of Freeway Acceleration Speed-Change Lanes". Transportation Research Board (2309), 311.

HCM (2000). "Highway Capacity Manual". Washington, D.C: Transportation Research Board, National Research Council.

Ibrahim, S. E.-B., \& Sayed, T. (2011). "Developing safety performance functions incorporating reliability-based risk measures". Accident Analysis and Prevention, $43(6), 2153-2159$.

Kaiser, H. \& Dickman, K., 1962. "Sample and Population Score Matrices and Sample Correlation Matrices from an Arbitrary Population Correlation Matrix". Psychometrik, 27(2), pp. 179-182.

Koepke, F. J. (1993). "Ramp Exit/Entrance Design--Taper Versus Parallel and Critical Dimensions". Transportation Research Board (1385), 126-132.

Low, B. K. \& Tang, W. H., 1997. "Efficient Relaiability Evaluation Using Spreadsheet”. Journal of Engineering mechanics, 123(7), pp. 749-752. 
Mahadevan, S. \& Haldar, A., 2000. "Probability, Reliability, and Statistical Methods in Engineering Design”. John Wiley Sons Inc. NewYork.

Pilko, P., Bared, J. G., Edara, P. K., \& Kim, T. (2012). "Safety Assessment of Interchange Spacing on Urban Freeways". Office of Research, Development, and Technology, Office of Safety. Federal Highway Adminstration.

Rojas, M. A. \& Garcia, A.G. (2010). "Deceleration Lane Length Evaluation Based on A new Conflict Indicator”. 4th International Symposium on Highway Geometric Design (pp. 1-11). Valencia: International Symposium on Highway Geometric Design.

Sarhan, M. E. (2004). "Safety Performance of Freeway Merge and Diverge Areas". Department of Civil and Environmental Engineerin, Carleton University. Ottawa, Ontario, Canada.

Sarhan, M. \& Hassan, Y., 2008. "Risk-Based Approach for Highway Geometric Design”. International Journal of Advances in Transportation Studies, Volume XV, pp. 3748.

Sarhan, M. \& Hassan, Y., 2008. “Three-Dimensional, Probabilistic Highway Design: Sight Distance Application”. Transportation Research Record, Volume 2060, pp. $10-18$.

Sarhan, M., \& Hassan, Y. (2011). "Reliability Based Three-Dimensional Design of Horizontal Lateral Clearance”. Canadian Journal of Civil Engineering (38), 900908. 
Sarhan, M., Hassan, Y., \& Abd El Halim, A. (2008). "Safety Performance of Freeway Sections and Relation to Length of Speed Change Lanes". Canadian Journal of Civil Engineering , 34 (5), 531-541.

Shin, J., \& Lee, I. (2013). "Reliability-Based Design Optimization of Highway Horizontal Curves Based on First Order Reliability". 10th World Congress on Structural and Multidisciplinary Optimization (pp. 19-24). Florida: World Congress on Structural and Multidisciplinary Optimization.

Singh, V., Jain, S. \& Tyagi, A., 2007. "Risk and Reliability Analysis: A Handbook for Civil and Environmental Engineers". American Society of Civil Engineers. Reston.

TAC. (1999). "Geometric Design Guide for Canadian Roads".Transportation Association of Canada. Ottawa, Ontario, Canada.

Torbic, D. J., Hutton, J. M., Bokenkroger, C. D., Harwood, D. W., Gilmore, D. K., Knoshaug, M. M., et al. (2012). "NCHRP Report 730; Design Guidance for Freeway Mainline Ramp Terminals". National Cooperative Highway Research Program, Transportation Research Board. Washington, D.C.

“The 2010 Highway Capacity Manual”. (2010). Transportation Research Center. Retrieved from Transportation Research Center: http://trc.ce.ufl.edu/news_and_events/hcm2010_workshop_washburn.pdf

“Analysis of Freeway Weaving Sections”. (2008). National Research Council. Retrieved from Transportation Research Board of the National Academy of Science: 
http://www.hcm2010.org/system/datas/69/original/NCHRP\%20375\%20Final\%20Report.pdf

Wang, Z., Lu, J. J., \& Zhang, Z. (2009). "Exploring Impacts of Factors Contributing to Injury Severity at Freeway Exit Ramps". Transportation Research Board: Journal of the Transportation Research Board (2012), 43-52.

You, K., Sun, L., \& Gu, W. (2012). "Reliability-Based Risk Analysis of Roadway Horizontal Curves”. Journal of Transportation Engineering , 1071-1081.

Zhao, Y.-G., \& Ono, T. (1999). "A general procedure for First/second-order reliability method (FORM/SORM)”. Structural Safety, 21, 95-112.

Zheng, Z. R. (1997).” Application of Reliability Theory to Highway Geometric Design”. Department of Civil Engineering, University of British Colombia. British Colombia. 
Appendices 


\section{Appendix 1: Summary of Geometric Data of Study}

Sites 
Table 1: Geometric Characteristics of Study Sites

\begin{tabular}{|c|c|c|c|c|c|c|c|c|}
\hline SCL Name & SCL Length (m) & $\begin{array}{l}\text { Transition } \\
\text { Length (m) }\end{array}$ & $\begin{array}{l}\text { SCL } \\
\text { Type }\end{array}$ & $\begin{array}{l}\text { Ramp } \\
\text { Grade }\end{array}$ & $\begin{array}{l}\text { Ramp } \\
\text { Width } \\
\text { (m) }\end{array}$ & $\begin{array}{c}\text { Freeway } \\
\text { Basic } \\
\text { Lanes }\end{array}$ & $\begin{array}{l}\text { Divergence } \\
\text { Angle } \\
\text { (degrees) }\end{array}$ & $\begin{array}{c}\text { Ramp } \\
\text { Advisory } \\
\text { Speed } \\
(\mathrm{km} / \mathrm{h})\end{array}$ \\
\hline Island Park Drive (E-N) & 58 & 22 & Taper & Up & 6.8 & 4 & 18 & 40 \\
\hline Parkdale Ave. (W-NS) & 179 & 41 & Taper & $\mathrm{Up}$ & 6.1 & 4 & 12 & 50 \\
\hline Bronson Ave. (W-NS) & 212 & 13 & Taper & Up & 6.1 & 4 & 11 & 40 \\
\hline Moodie Drive (W-NS) & 292 & 83 & Parallel & Down & 4.8 & 2 & 7 & 50 \\
\hline Terry Fox Drive (E-NS) & 388 & 112 & Parallel & Down & 4.6 & 2 & 6.5 & 50 \\
\hline Carp Road (E-NS) & 430 & 49 & Parallel & Down & 5.2 & 2 & 4 & 70 \\
\hline Terry Fox Drive (W-NS) & 446 & 124 & Parallel & Down & 4.6 & 2 & 6 & 60 \\
\hline
\end{tabular}




\section{Appendix 2: Matlab Script Estimating Deceleration \\ Point}




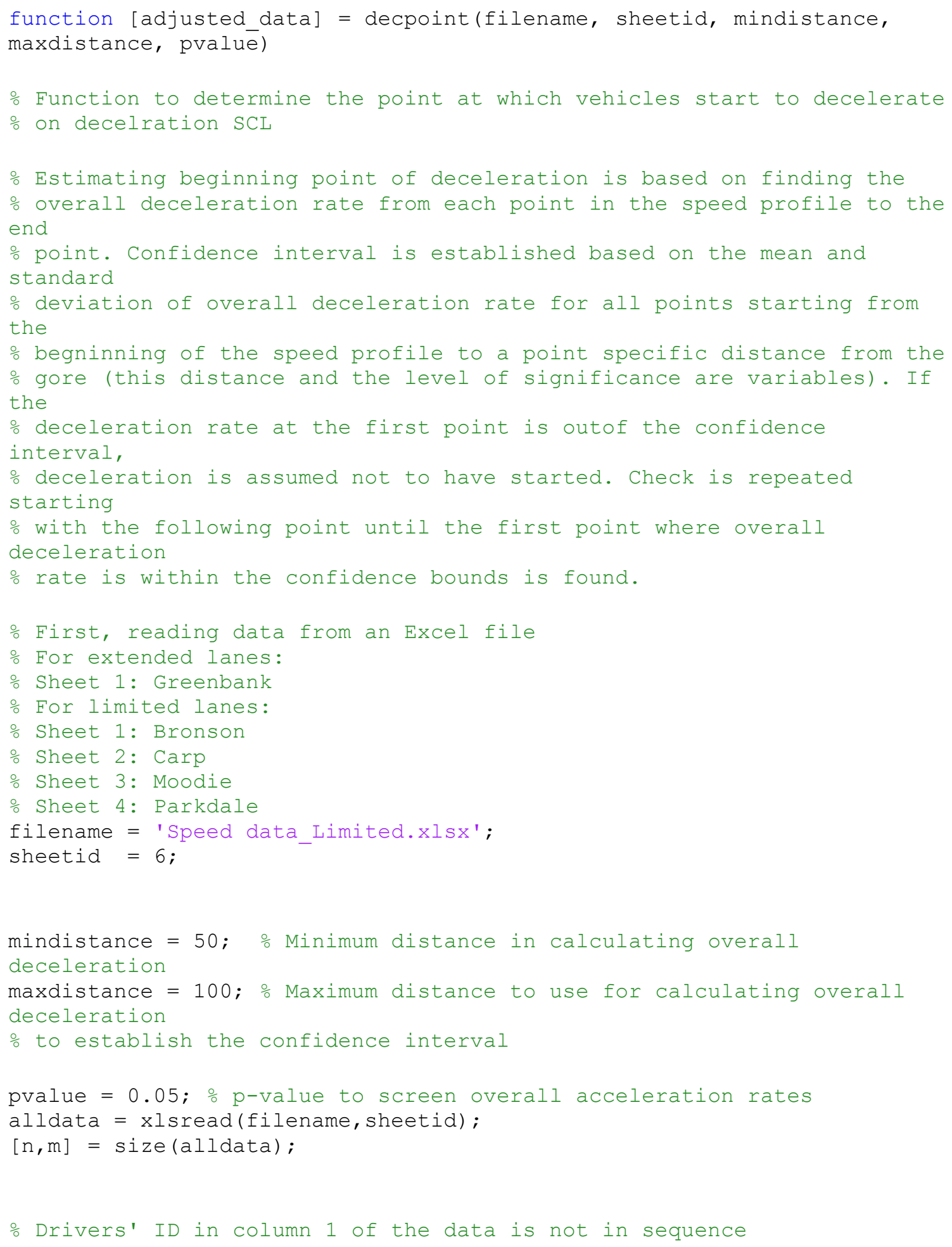




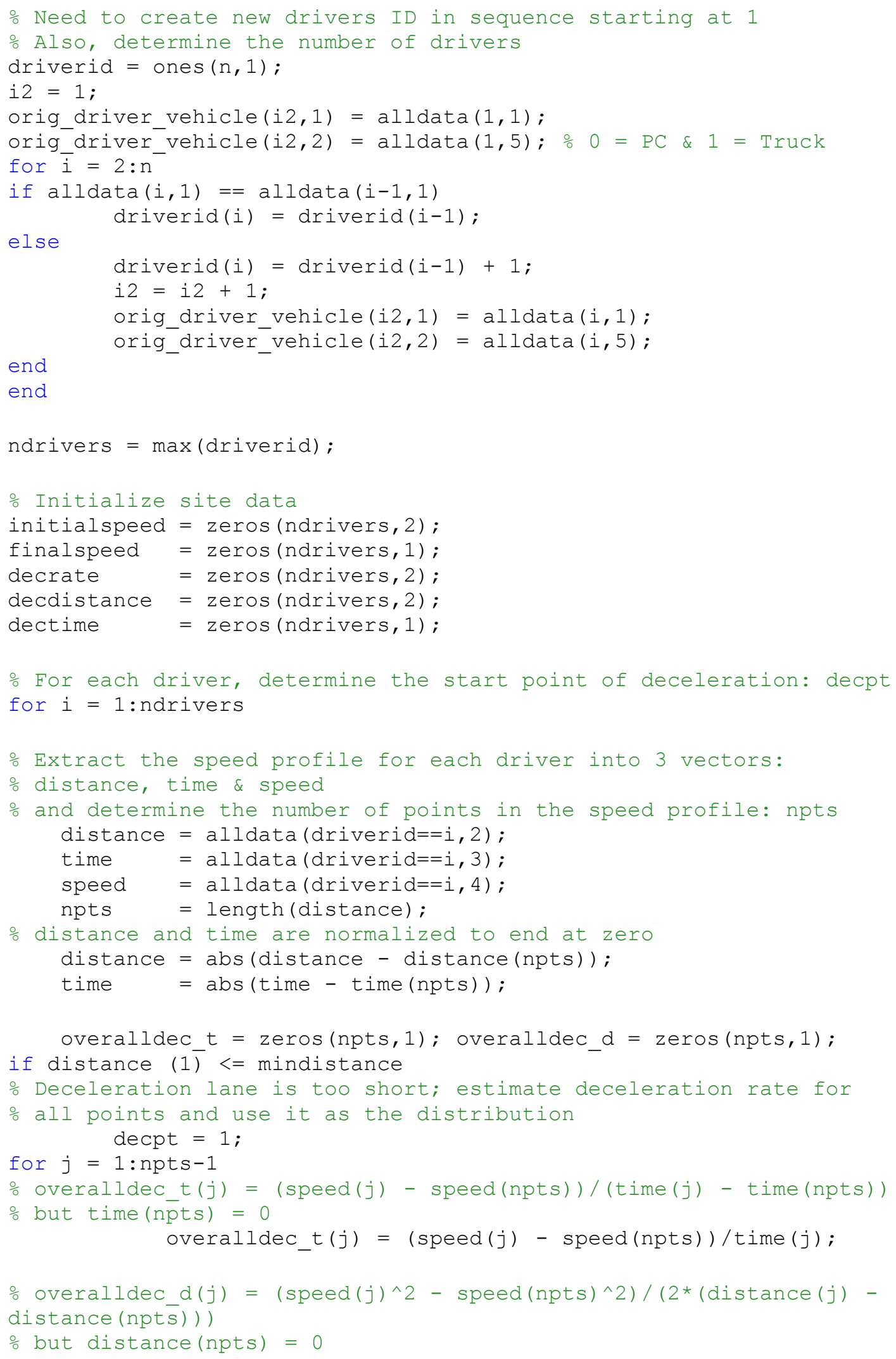

end 


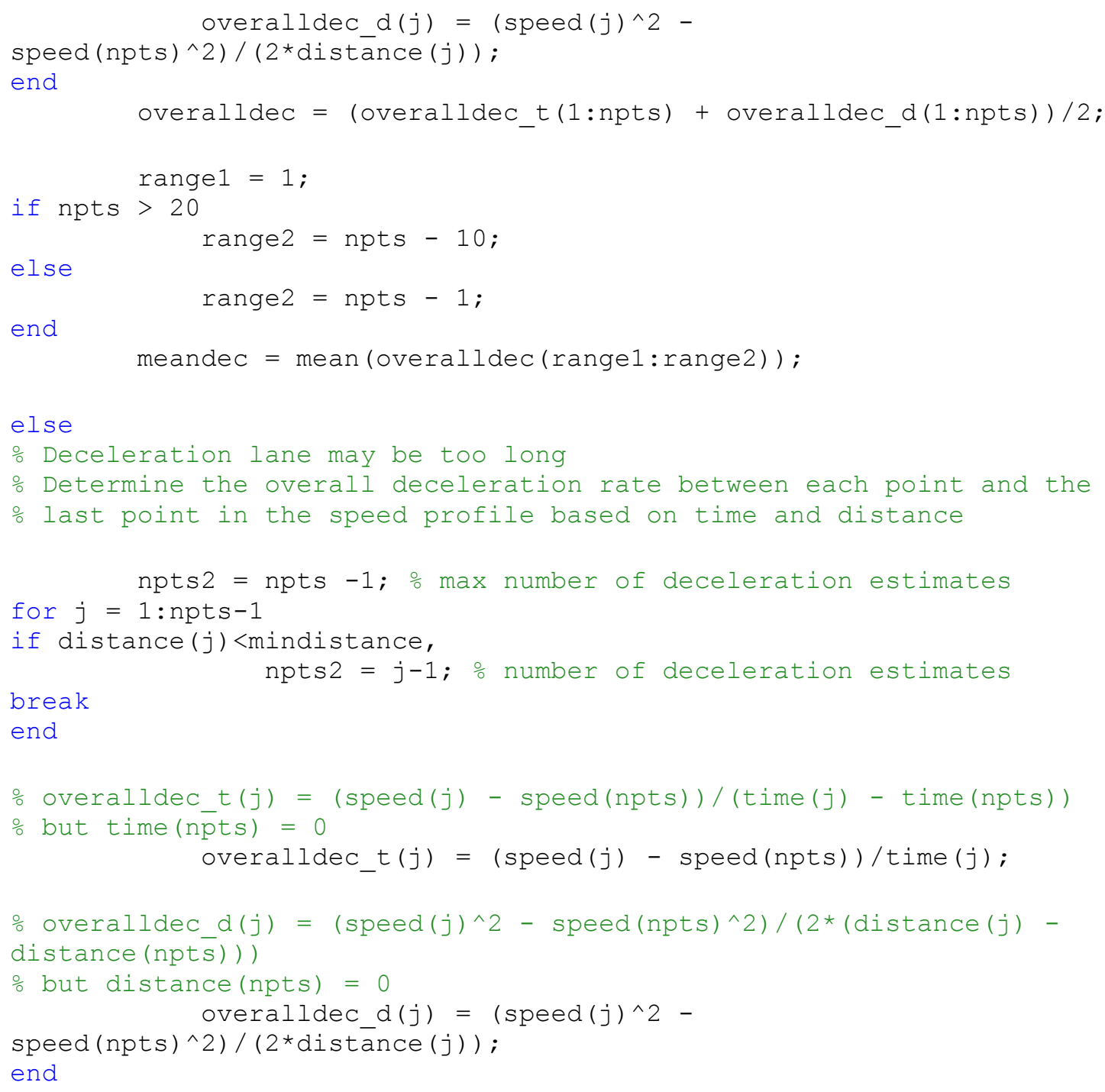




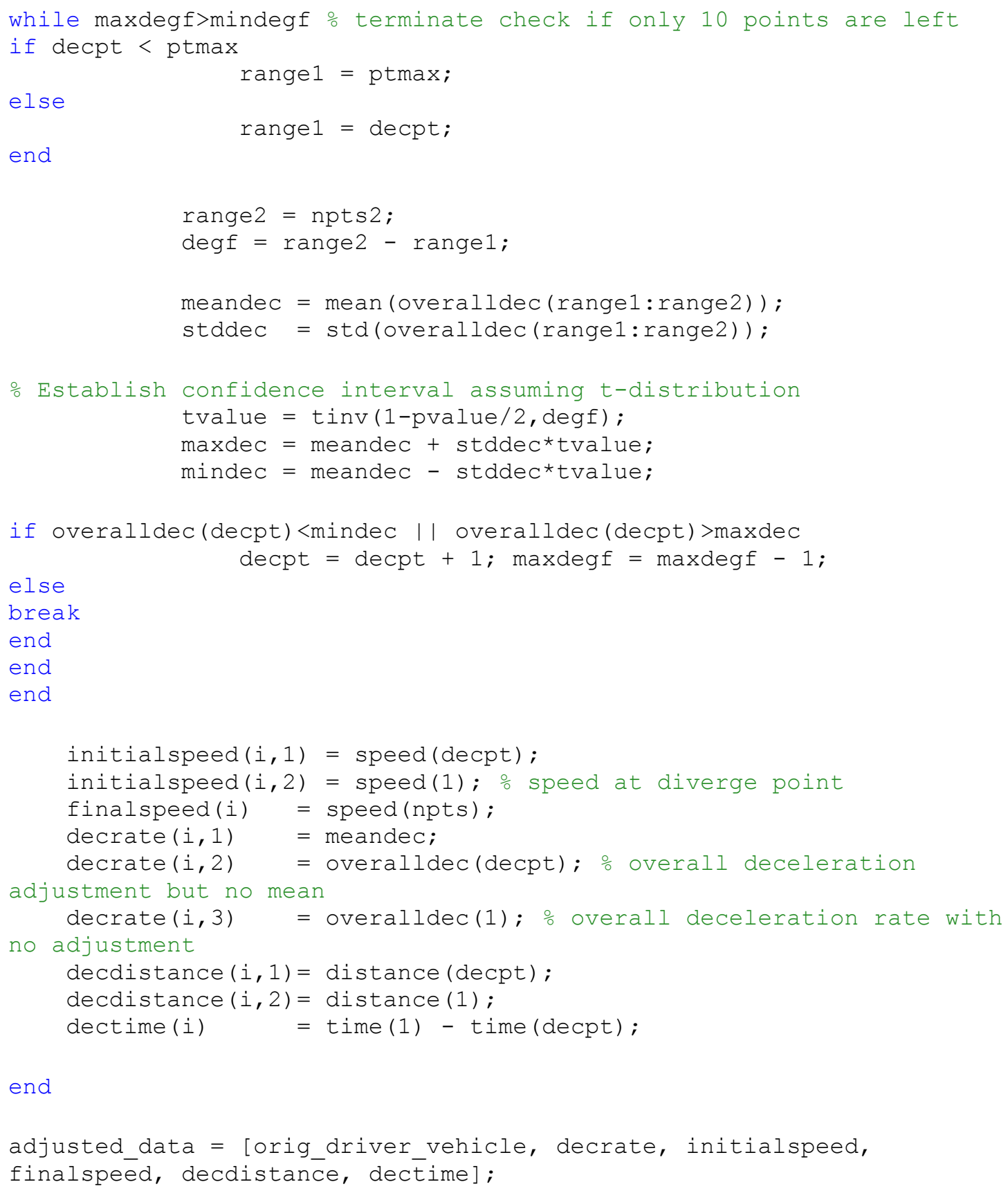




\title{
Appendix 3: Final Database After Reprocessing the
}

\author{
Data
}


Table 2: Island Park Drive (E-N).

\begin{tabular}{|c|c|c|c|c|c|c|c|c|c|c|c|}
\hline $\begin{array}{c}\text { Truck } \\
\mathrm{s}\end{array}$ & \begin{tabular}{|c} 
Adjusted \\
Overall \\
Deceleratio \\
$\mathrm{n}$
\end{tabular} & \begin{tabular}{|c|} 
Original \\
Overall \\
Deceleratio \\
$\mathrm{n}$
\end{tabular} & $\begin{array}{c}\text { Initial } \\
\text { Spee } \\
d\end{array}$ & $\begin{array}{c}\text { Diverg } \\
\mathrm{e} \\
\text { Speed }\end{array}$ & $\begin{array}{c}\text { Final } \\
\text { Spee } \\
\mathrm{d}\end{array}$ & $\begin{array}{l}\text { Deceleratio } \\
\text { n Distance }\end{array}$ & $\begin{array}{c}\text { Deceleratio } \\
\text { n Length }\end{array}$ & $\begin{array}{c}\text { Deceleratio } \\
\text { n Time }\end{array}$ & \begin{tabular}{|c|} 
Distance \\
to \\
Decelerat \\
$\mathrm{e}$
\end{tabular} & $\begin{array}{c}\mathrm{SCL} \\
\text { Lengt } \\
\mathrm{h}\end{array}$ & $\begin{array}{c}\text { Utilizatio } \\
\text { n Rate }\end{array}$ \\
\hline 0 & 1.46 & 1.46 & 22.31 & 22.31 & 20.07 & 31.51 & 31.51 & 0.00 & 0.00 & 58 & 0.54 \\
\hline 0 & 0.28 & 0.28 & 23.95 & 23.95 & 23.38 & 47.07 & 47.07 & 0.00 & 0.00 & 58 & 0.81 \\
\hline 0 & 0.39 & 0.39 & 21.78 & 21.78 & 21.30 & 26.59 & 26.59 & 0.00 & 0.00 & 58 & 0.46 \\
\hline 0 & 0.76 & 0.76 & 22.59 & 22.59 & 21.16 & 40.86 & 40.86 & 0.00 & 0.00 & 58 & 0.70 \\
\hline 0 & 0.24 & 0.24 & 19.18 & 19.18 & 18.90 & 22.49 & 22.49 & 0.00 & 0.00 & 58 & 0.39 \\
\hline 0 & 0.43 & 0.43 & 20.58 & 20.58 & 20.04 & 25.26 & 25.26 & 0.00 & 0.00 & 58 & 0.44 \\
\hline 0 & 0.49 & 0.49 & 20.72 & 20.72 & 19.63 & 44.91 & 44.91 & 0.00 & 0.00 & 58 & 0.77 \\
\hline 0 & 0.40 & 0.40 & 19.49 & 19.49 & 18.79 & 33.72 & 33.72 & 0.00 & 0.00 & 58 & 0.58 \\
\hline 0 & 0.78 & 0.78 & 22.95 & 22.95 & 21.52 & 39.96 & 39.96 & 0.00 & 0.00 & 58 & 0.69 \\
\hline 0 & 0.64 & 0.64 & 22.02 & 22.02 & 21.50 & 17.64 & 17.64 & 0.00 & 0.00 & 58 & 0.30 \\
\hline 0 & 0.20 & 0.20 & 21.69 & 21.69 & 21.35 & 36.98 & 36.98 & 0.00 & 0.00 & 58 & 0.64 \\
\hline 0 & 0.46 & 0.46 & 25.70 & 25.70 & 25.14 & 31.04 & 31.04 & 0.00 & 0.00 & 58 & 0.54 \\
\hline 0 & 0.28 & 0.28 & 23.11 & 23.11 & 22.72 & 33.39 & 33.39 & 0.00 & 0.00 & 58 & 0.58 \\
\hline 0 & 0.44 & 0.44 & 21.13 & 21.13 & 20.63 & 23.52 & 23.52 & 0.00 & 0.00 & 58 & 0.41 \\
\hline 0 & 0.94 & 0.94 & 21.77 & 21.77 & 21.02 & 17.14 & 17.14 & 0.00 & 0.00 & 58 & 0.30 \\
\hline 0 & 0.26 & 0.26 & 24.42 & 24.42 & 24.16 & 24.00 & 24.00 & 0.00 & 0.00 & 58 & 0.41 \\
\hline 0 & 0.43 & 0.43 & 20.80 & 20.80 & 20.18 & 29.38 & 29.38 & 0.00 & 0.00 & 58 & 0.51 \\
\hline 0 & 0.43 & 0.43 & 20.30 & 20.30 & 19.43 & 39.83 & 39.83 & 0.00 & 0.00 & 58 & 0.69 \\
\hline 0 & 0.21 & 0.21 & 21.64 & 21.64 & 21.32 & 32.94 & 32.94 & 0.00 & 0.00 & 58 & 0.57 \\
\hline 0 & 0.27 & 0.27 & 21.30 & 21.30 & 20.88 & 33.54 & 33.54 & 0.00 & 0.00 & 58 & 0.58 \\
\hline 0 & 0.44 & 0.44 & 20.69 & 20.69 & 20.07 & 28.86 & 28.86 & 0.00 & 0.00 & 58 & 0.50 \\
\hline 0 & 0.43 & 0.43 & 25.01 & 25.01 & 24.27 & 41.71 & 41.71 & 0.00 & 0.00 & 58 & 0.72 \\
\hline 0 & 0.85 & 0.85 & 24.74 & 24.74 & 22.77 & 54.45 & 54.45 & 0.00 & 0.00 & 58 & 0.94 \\
\hline 0 & 0.35 & 0.35 & 22.33 & 22.33 & 21.85 & 29.61 & 29.61 & 0.00 & 0.00 & 58 & 0.51 \\
\hline
\end{tabular}




\begin{tabular}{|c|c|c|c|c|c|c|c|c|c|c|c|}
\hline 0 & 0.44 & 0.44 & 18.69 & 18.69 & 17.76 & 38.41 & 38.41 & 0.00 & 0.00 & 58 & 0.66 \\
\hline 0 & 0.51 & 0.51 & 18.19 & 18.19 & 17.04 & 39.03 & 39.03 & 0.00 & 0.00 & 58 & 0.67 \\
\hline 0 & 0.57 & 0.57 & 17.68 & 17.68 & 16.34 & 39.28 & 39.28 & 0.00 & 0.00 & 58 & 0.68 \\
\hline 0 & 0.64 & 0.64 & 20.69 & 20.69 & 19.88 & 25.21 & 25.21 & 0.00 & 0.00 & 58 & 0.43 \\
\hline 0 & 0.18 & 0.18 & 20.02 & 20.02 & 19.65 & 39.66 & 39.66 & 0.00 & 0.00 & 58 & 0.68 \\
\hline 0 & 0.53 & 0.53 & 20.00 & 20.00 & 18.93 & 38.73 & 38.73 & 0.00 & 0.00 & 58 & 0.67 \\
\hline 0 & 0.58 & 0.58 & 19.99 & 19.99 & 19.24 & 25.11 & 25.11 & 0.00 & 0.00 & 58 & 0.43 \\
\hline 0 & 0.32 & 0.32 & 18.38 & 18.38 & 17.76 & 35.45 & 35.45 & 0.00 & 0.00 & 58 & 0.61 \\
\hline 0 & 0.56 & 0.56 & 20.41 & 20.41 & 20.02 & 13.81 & 13.81 & 0.00 & 0.00 & 58 & 0.24 \\
\hline 0 & 0.62 & 0.62 & 19.16 & 19.16 & 18.18 & 29.35 & 29.35 & 0.00 & 0.00 & 58 & 0.51 \\
\hline 0 & 0.47 & 0.47 & 18.02 & 18.02 & 17.04 & 36.30 & 36.30 & 0.00 & 0.00 & 58 & 0.63 \\
\hline 0 & 0.57 & 0.57 & 20.05 & 20.05 & 19.60 & 15.42 & 15.42 & 0.00 & 0.00 & 58 & 0.27 \\
\hline 0 & 0.73 & 0.73 & 15.18 & 15.18 & 13.36 & 34.64 & 34.64 & 0.00 & 0.00 & 58 & 0.60 \\
\hline 0 & 0.19 & 0.19 & 17.27 & 17.27 & 16.90 & 33.48 & 33.48 & 0.00 & 0.00 & 58 & 0.58 \\
\hline 0 & 0.69 & 0.69 & 19.02 & 19.02 & 18.15 & 23.02 & 23.02 & 0.00 & 0.00 & 58 & 0.40 \\
\hline 0 & 0.54 & 0.54 & 21.56 & 21.56 & 20.52 & 40.08 & 40.08 & 0.00 & 0.00 & 58 & 0.69 \\
\hline 0 & 0.71 & 0.71 & 17.51 & 17.51 & 16.48 & 24.67 & 24.67 & 0.00 & 0.00 & 58 & 0.43 \\
\hline 0 & 0.42 & 0.42 & 19.91 & 19.91 & 18.93 & 44.96 & 44.96 & 0.00 & 0.00 & 58 & 0.78 \\
\hline 0 & 0.49 & 0.49 & 16.29 & 16.29 & 15.14 & 36.94 & 36.94 & 0.00 & 0.00 & 58 & 0.64 \\
\hline 0 & 0.95 & 0.95 & 22.37 & 22.37 & 20.38 & 44.39 & 44.39 & 0.00 & 0.00 & 58 & 0.77 \\
\hline 0 & 0.81 & 0.81 & 19.55 & 19.55 & 18.15 & 32.56 & 32.56 & 0.00 & 0.00 & 58 & 0.56 \\
\hline 0 & 0.48 & 0.48 & 20.60 & 20.60 & 20.07 & 22.30 & 22.30 & 0.00 & 0.00 & 58 & 0.38 \\
\hline 0 & 0.27 & 0.27 & 19.19 & 19.19 & 18.73 & 31.01 & 31.01 & 0.00 & 0.00 & 58 & 0.53 \\
\hline 0 & 0.37 & 0.37 & 19.22 & 19.22 & 18.26 & 48.40 & 48.40 & 0.00 & 0.00 & 58 & 0.83 \\
\hline 0 & 0.27 & 0.27 & 20.66 & 20.66 & 20.25 & 30.56 & 30.56 & 0.00 & 0.00 & 58 & 0.53 \\
\hline 0 & 0.26 & 0.26 & 19.99 & 19.99 & 19.63 & 27.69 & 27.69 & 0.00 & 0.00 & 58 & 0.48 \\
\hline 0 & 0.95 & 0.95 & 19.69 & 19.69 & 18.40 & 25.79 & 25.79 & 0.00 & 0.00 & 58 & 0.44 \\
\hline
\end{tabular}




\begin{tabular}{|c|c|c|c|c|c|c|c|c|c|c|c|}
\hline 0 & 0.28 & 0.28 & 21.28 & 21.28 & 20.88 & 29.81 & 29.81 & 0.00 & 0.00 & 58 & 0.51 \\
\hline 0 & 0.46 & 0.46 & 21.41 & 21.41 & 20.77 & 29.59 & 29.59 & 0.00 & 0.00 & 58 & 0.51 \\
\hline 0 & 0.52 & 0.52 & 17.65 & 17.65 & 17.29 & 12.07 & 12.07 & 0.00 & 0.00 & 58 & 0.21 \\
\hline 0 & 0.51 & 0.51 & 19.30 & 19.30 & 18.48 & 30.00 & 30.00 & 0.00 & 0.00 & 58 & 0.52 \\
\hline 0 & 0.29 & 0.29 & 17.32 & 17.32 & 17.09 & 13.05 & 13.05 & 0.00 & 0.00 & 58 & 0.22 \\
\hline 0 & 1.42 & 1.42 & 23.14 & 23.14 & 20.41 & 40.94 & 40.94 & 0.00 & 0.00 & 58 & 0.71 \\
\hline 0 & 0.59 & 0.59 & 19.86 & 19.86 & 18.85 & 32.87 & 32.87 & 0.00 & 0.00 & 58 & 0.57 \\
\hline 0 & 0.44 & 0.44 & 21.97 & 21.97 & 21.24 & 36.20 & 36.20 & 0.00 & 0.00 & 58 & 0.62 \\
\hline 0 & 0.56 & 0.56 & 20.33 & 20.33 & 19.43 & 31.85 & 31.85 & 0.00 & 0.00 & 58 & 0.55 \\
\hline 0 & 0.42 & 0.42 & 21.11 & 21.11 & 20.32 & 38.84 & 38.84 & 0.00 & 0.00 & 58 & 0.67 \\
\hline 0 & 1.22 & 1.22 & 19.83 & 19.83 & 17.62 & 34.45 & 34.45 & 0.00 & 0.00 & 58 & 0.59 \\
\hline 0 & 1.35 & 1.35 & 20.44 & 20.44 & 17.18 & 44.39 & 44.39 & 0.00 & 0.00 & 58 & 0.77 \\
\hline 0 & 0.97 & 0.97 & 22.20 & 22.20 & 20.46 & 37.86 & 37.86 & 0.00 & 0.00 & 58 & 0.65 \\
\hline 0 & 0.71 & 0.71 & 21.08 & 21.08 & 19.88 & 34.32 & 34.32 & 0.00 & 0.00 & 58 & 0.59 \\
\hline 0 & 0.08 & 0.08 & 19.52 & 19.52 & 19.38 & 38.26 & 38.26 & 0.00 & 0.00 & 58 & 0.66 \\
\hline 0 & 0.60 & 0.60 & 21.47 & 21.47 & 20.54 & 32.20 & 32.20 & 0.00 & 0.00 & 58 & 0.56 \\
\hline 0 & 0.43 & 0.43 & 21.89 & 21.89 & 21.24 & 32.81 & 32.81 & 0.00 & 0.00 & 58 & 0.57 \\
\hline 0 & 0.61 & 0.61 & 17.27 & 17.27 & 15.12 & 56.23 & 56.23 & 0.00 & 0.00 & 58 & 0.97 \\
\hline 0 & 0.50 & 0.50 & 19.69 & 19.69 & 19.01 & 25.55 & 25.55 & 0.00 & 0.00 & 58 & 0.44 \\
\hline 0 & 0.29 & 0.29 & 19.71 & 19.71 & 19.43 & 18.75 & 18.75 & 0.00 & 0.00 & 58 & 0.32 \\
\hline 0 & 0.52 & 0.52 & 21.19 & 21.19 & 20.46 & 29.50 & 29.50 & 0.00 & 0.00 & 58 & 0.51 \\
\hline 0 & 0.57 & 0.57 & 24.54 & 24.54 & 23.61 & 39.34 & 39.34 & 0.00 & 0.00 & 58 & 0.68 \\
\hline 0 & 0.64 & 0.64 & 19.86 & 19.86 & 18.57 & 37.99 & 37.99 & 0.00 & 0.00 & 58 & 0.65 \\
\hline 0 & 1.22 & 1.22 & 21.50 & 21.50 & 20.35 & 19.19 & 19.19 & 0.00 & 0.00 & 58 & 0.33 \\
\hline 0 & 0.39 & 0.39 & 21.11 & 21.11 & 20.54 & 30.35 & 30.35 & 0.00 & 0.00 & 58 & 0.52 \\
\hline 0 & 0.23 & 0.23 & 14.73 & 14.73 & 14.23 & 31.00 & 31.00 & 0.00 & 0.00 & 58 & 0.53 \\
\hline 0 & 0.23 & 0.23 & 20.69 & 20.69 & 20.21 & 42.63 & 42.63 & 0.00 & 0.00 & 58 & 0.74 \\
\hline
\end{tabular}




\begin{tabular}{|c|c|c|c|c|c|c|c|c|c|c|c|}
\hline 0 & 0.56 & 0.56 & 19.25 & 19.25 & 17.90 & 44.52 & 44.52 & 0.00 & 0.00 & 58 & 0.77 \\
\hline 0 & 0.34 & 0.34 & 24.79 & 24.79 & 24.25 & 38.21 & 38.21 & 0.00 & 0.00 & 58 & 0.66 \\
\hline 0 & 0.54 & 0.54 & 23.56 & 23.56 & 22.66 & 39.16 & 39.16 & 0.00 & 0.00 & 58 & 0.68 \\
\hline 0 & 0.22 & 0.22 & 17.15 & 17.15 & 16.76 & 29.67 & 29.67 & 0.00 & 0.00 & 58 & 0.51 \\
\hline 0 & 1.17 & 1.17 & 22.75 & 22.75 & 20.49 & 41.44 & 41.44 & 0.00 & 0.00 & 58 & 0.71 \\
\hline 0 & 0.46 & 0.46 & 18.88 & 18.88 & 17.96 & 36.84 & 36.84 & 0.00 & 0.00 & 58 & 0.64 \\
\hline 0 & 0.62 & 0.62 & 23.00 & 23.00 & 21.91 & 39.11 & 39.11 & 0.00 & 0.00 & 58 & 0.67 \\
\hline $\begin{array}{c}\text { Mea } \\
\mathrm{n}\end{array}$ & 0.54 & 0.54 & 20.58 & 20.58 & 19.66 & 33.08 & 33.08 & 0.00 & 0.00 & 58 & 0.57 \\
\hline Std & 0.29 & 0.29 & 2.16 & 2.16 & 2.21 & 8.86 & 8.86 & 0.00 & 0.00 & 0 & 0.15 \\
\hline
\end{tabular}


Table 3: Parkdale Ave. (W-NS).

\begin{tabular}{|c|c|c|c|c|c|c|c|c|c|c|c|c|}
\hline $\begin{array}{l}\text { Tru } \\
\text { cks }\end{array}$ & $\begin{array}{c}\text { Mean } \\
\text { Decelerati } \\
\text { on }\end{array}$ & \begin{tabular}{|} 
Adjusted \\
Overall \\
Decelerati \\
on
\end{tabular} & \begin{tabular}{|c|} 
Original \\
Overall \\
Decelerati \\
on
\end{tabular} & $\begin{array}{l}\text { Initial } \\
\text { Speed }\end{array}$ & $\begin{array}{c}\text { Diverg } \\
\mathrm{e} \\
\text { Speed }\end{array}$ & $\begin{array}{l}\text { Final } \\
\text { Speed }\end{array}$ & $\begin{array}{l}\text { Decelera } \\
\text { tion } \\
\text { Distance }\end{array}$ & $\begin{array}{l}\text { Decelera } \\
\text { tion } \\
\text { Length }\end{array}$ & $\begin{array}{l}\text { Decelerati } \\
\text { on Time }\end{array}$ & $\begin{array}{c}\text { Distance } \\
\text { to } \\
\text { Decelera } \\
\text { te }\end{array}$ & $\begin{array}{c}\mathrm{SCL} \\
\text { Lengt } \\
\mathrm{h}\end{array}$ & $\begin{array}{l}\text { Utilizati } \\
\text { on Rate }\end{array}$ \\
\hline 0 & 0.84 & 0.80 & 0.80 & 22.21 & 22.21 & 19.18 & 77.49 & 77.49 & 0.00 & 0.00 & 179 & 0.43 \\
\hline 0 & 0.76 & 0.79 & 0.79 & 22.53 & 22.53 & 19.40 & 82.08 & 82.08 & 0.00 & 0.00 & 179 & 0.46 \\
\hline 0 & 0.89 & 0.96 & 0.96 & 23.04 & 23.04 & 21.07 & 44.45 & 44.45 & 0.00 & 0.00 & 179 & 0.25 \\
\hline 0 & 0.44 & 0.40 & 0.29 & 21.15 & 20.82 & 19.62 & 78.36 & 83.78 & 0.26 & 5.42 & 179 & 0.44 \\
\hline 0 & 1.27 & 1.05 & 1.05 & 22.51 & 22.51 & 17.14 & 101.85 & 101.85 & 0.00 & 0.00 & 179 & 0.57 \\
\hline 0 & 1.85 & 1.65 & 1.65 & 27.34 & 27.34 & 21.94 & 79.81 & 79.81 & 0.00 & 0.00 & 179 & 0.45 \\
\hline 0 & 0.46 & 0.48 & 0.48 & 25.25 & 25.25 & 24.40 & 43.62 & 43.62 & 0.00 & 0.00 & 179 & 0.24 \\
\hline 0 & 1.44 & 1.37 & 1.22 & 24.62 & 24.50 & 19.79 & 77.10 & 84.58 & 0.30 & 7.47 & 179 & 0.43 \\
\hline 0 & 1.44 & 1.68 & 1.68 & 28.39 & 28.39 & 23.78 & 70.28 & 70.28 & 0.00 & 0.00 & 179 & 0.39 \\
\hline 0 & 1.44 & 1.35 & 1.35 & 23.36 & 23.36 & 19.65 & 58.41 & 58.41 & 0.00 & 0.00 & 179 & 0.33 \\
\hline 0 & 1.12 & 1.01 & 1.01 & 23.07 & 23.07 & 18.93 & 85.93 & 85.93 & 0.00 & 0.00 & 179 & 0.48 \\
\hline 0 & 1.12 & 1.17 & 1.17 & 24.96 & 24.96 & 21.91 & 60.11 & 60.11 & 0.00 & 0.00 & 179 & 0.34 \\
\hline 0 & 1.12 & 0.50 & 0.50 & 22.82 & 22.82 & 21.30 & 67.15 & 67.15 & 0.00 & 0.00 & 179 & 0.38 \\
\hline 0 & 1.12 & 1.49 & 1.49 & 22.89 & 22.89 & 18.48 & 59.80 & 59.80 & 0.00 & 0.00 & 179 & 0.33 \\
\hline 0 & 1.12 & 1.59 & 1.59 & 25.52 & 25.52 & 19.85 & 79.34 & 79.34 & 0.00 & 0.00 & 179 & 0.44 \\
\hline 0 & 0.88 & 0.80 & 0.55 & 28.08 & 27.62 & 26.07 & 67.99 & 75.14 & 0.25 & 7.16 & 179 & 0.38 \\
\hline 0 & 1.80 & 1.51 & 1.51 & 24.00 & 24.00 & 18.79 & 72.90 & 72.90 & 0.00 & 0.00 & 179 & 0.41 \\
\hline 0 & 0.62 & 0.57 & 0.57 & 23.21 & 23.21 & 21.35 & 72.34 & 72.34 & 0.00 & 0.00 & 179 & 0.40 \\
\hline 0 & 1.48 & 1.25 & 1.25 & 25.38 & 25.38 & 21.21 & 77.64 & 77.64 & 0.00 & 0.00 & 179 & 0.43 \\
\hline 0 & 0.54 & 0.53 & 0.62 & 20.91 & 21.90 & 19.09 & 67.44 & 91.63 & 1.15 & 24.19 & 179 & 0.38 \\
\hline 0 & 1.07 & 1.13 & 1.13 & 23.69 & 23.69 & 22.39 & 26.30 & 26.30 & 0.00 & 0.00 & 179 & 0.15 \\
\hline 0 & 1.07 & 1.61 & 1.61 & 23.85 & 23.85 & 18.59 & 68.46 & 68.46 & 0.00 & 0.00 & 179 & 0.38 \\
\hline 0 & 1.07 & 1.10 & 1.10 & 23.88 & 23.88 & 20.63 & 65.01 & 65.01 & 0.00 & 0.00 & 179 & 0.36 \\
\hline 0 & 0.85 & 0.78 & 0.71 & 27.96 & 28.01 & 25.62 & 79.50 & 89.36 & 0.35 & 9.86 & 179 & 0.44 \\
\hline 0 & 0.29 & 0.34 & 0.34 & 21.78 & 21.78 & 21.10 & 43.17 & 43.17 & 0.00 & 0.00 & 179 & 0.24 \\
\hline 0 & 0.29 & 1.46 & 1.46 & 25.54 & 25.54 & 22.00 & 56.67 & 56.67 & 0.00 & 0.00 & 179 & 0.32 \\
\hline 0 & 0.70 & 0.55 & 0.55 & 21.03 & 21.03 & 19.01 & 73.80 & 73.80 & 0.00 & 0.00 & 179 & 0.41 \\
\hline 0 & 0.97 & 0.92 & 0.92 & 19.63 & 19.63 & 14.54 & 93.09 & 93.09 & 0.00 & 0.00 & 179 & 0.52 \\
\hline 0 & 0.63 & 0.62 & 0.58 & 20.83 & 20.78 & 18.67 & 68.20 & 71.35 & 0.16 & 3.15 & 179 & 0.38 \\
\hline 0 & 0.63 & 0.92 & 0.92 & 26.11 & 26.11 & 23.92 & 59.13 & 59.13 & 0.00 & 0.00 & 179 & 0.33 \\
\hline 0 & 0.72 & 0.68 & 0.55 & 23.76 & 23.47 & 21.24 & 82.27 & 90.74 & 0.36 & 8.47 & 179 & 0.46 \\
\hline 0 & 0.37 & 0.39 & 0.39 & 20.64 & 20.64 & 18.76 & 94.36 & 94.36 & 0.00 & 0.00 & 179 & 0.53 \\
\hline 0 & 0.37 & 0.89 & 0.89 & 21.82 & 21.82 & 19.40 & 55.12 & 55.12 & 0.00 & 0.00 & 179 & 0.31 \\
\hline 0 & 0.99 & 0.84 & 0.84 & 22.58 & 22.58 & 19.46 & 76.96 & 76.96 & 0.00 & 0.00 & 179 & 0.43 \\
\hline 0 & 0.99 & 1.00 & 1.00 & 19.50 & 19.50 & 16.47 & 53.18 & 53.18 & 0.00 & 0.00 & 179 & 0.30 \\
\hline
\end{tabular}




\begin{tabular}{|c|c|c|c|c|c|c|c|c|c|c|c|c|}
\hline 0 & 1.28 & 1.17 & 1.17 & 23.80 & 23.80 & 19.01 & 86.87 & 86.87 & 0.00 & 0.00 & 179 & 0.49 \\
\hline 0 & 0.62 & 0.61 & 0.61 & 21.46 & 21.46 & 19.15 & 76.85 & 76.85 & 0.00 & 0.00 & 179 & 0.43 \\
\hline 0 & 0.67 & 0.60 & 0.60 & 23.63 & 23.63 & 21.80 & 69.70 & 69.70 & 0.00 & 0.00 & 179 & 0.39 \\
\hline 0 & 1.21 & 1.15 & 1.15 & 21.90 & 21.90 & 16.33 & 90.64 & 90.64 & 0.00 & 0.00 & 179 & 0.51 \\
\hline 0 & 1.25 & 1.06 & 1.06 & 24.07 & 24.07 & 20.13 & 82.09 & 82.09 & 0.00 & 0.00 & 179 & 0.46 \\
\hline 0 & 0.80 & 0.69 & 0.69 & 20.80 & 20.80 & 18.56 & 64.14 & 64.14 & 0.00 & 0.00 & 179 & 0.36 \\
\hline 0 & 0.97 & 1.14 & 1.14 & 23.09 & 23.09 & 20.88 & 42.23 & 42.23 & 0.00 & 0.00 & 179 & 0.24 \\
\hline 0 & 0.49 & 0.39 & 0.39 & 21.96 & 21.96 & 20.52 & 78.35 & 78.35 & 0.00 & 0.00 & 179 & 0.44 \\
\hline 0 & 0.70 & 0.53 & 0.53 & 20.97 & 20.97 & 19.06 & 72.88 & 72.88 & 0.00 & 0.00 & 179 & 0.41 \\
\hline 0 & 1.75 & 1.16 & 1.16 & 25.67 & 25.67 & 23.34 & 49.00 & 49.00 & 0.00 & 0.00 & 179 & 0.27 \\
\hline 0 & 0.81 & 0.77 & 0.77 & 20.77 & 20.77 & 17.28 & 85.65 & 85.65 & 0.00 & 0.00 & 179 & 0.48 \\
\hline 0 & 1.14 & 0.97 & 0.97 & 25.94 & 25.94 & 22.89 & 76.37 & 76.37 & 0.00 & 0.00 & 179 & 0.43 \\
\hline 0 & 0.76 & 0.78 & 0.78 & 20.67 & 20.67 & 17.67 & 72.14 & 72.14 & 0.00 & 0.00 & 179 & 0.40 \\
\hline 0 & 0.62 & 0.61 & 0.61 & 18.54 & 18.54 & 14.99 & 96.52 & 96.52 & 0.00 & 0.00 & 179 & 0.54 \\
\hline 0 & 0.89 & 0.82 & 0.67 & 22.64 & 22.29 & 19.48 & 79.91 & 86.85 & 0.31 & 6.93 & 179 & 0.45 \\
\hline 0 & 0.66 & 0.65 & 0.65 & 18.48 & 18.48 & 14.82 & 92.37 & 92.37 & 0.00 & 0.00 & 179 & 0.52 \\
\hline 0 & 0.60 & 0.59 & 0.59 & 20.26 & 20.26 & 18.31 & 63.45 & 63.45 & 0.00 & 0.00 & 179 & 0.35 \\
\hline 0 & 0.53 & 0.50 & 0.50 & 23.21 & 23.21 & 21.66 & 68.88 & 68.88 & 0.00 & 0.00 & 179 & 0.38 \\
\hline 0 & 1.01 & 0.77 & 0.77 & 21.81 & 21.81 & 20.26 & 41.74 & 41.74 & 0.00 & 0.00 & 179 & 0.23 \\
\hline 0 & 1.13 & 1.17 & 1.01 & 28.78 & 28.86 & 25.88 & 66.89 & 80.13 & 0.46 & 13.24 & 179 & 0.37 \\
\hline 0 & 0.93 & 0.87 & 0.75 & 28.46 & 28.48 & 26.04 & 75.22 & 88.32 & 0.45 & 13.10 & 179 & 0.42 \\
\hline 0 & 0.61 & 0.50 & 0.50 & 23.26 & 23.26 & 21.77 & 66.03 & 66.03 & 0.00 & 0.00 & 179 & 0.37 \\
\hline 0 & 1.42 & 1.35 & 1.35 & 25.70 & 25.70 & 21.91 & 65.95 & 65.95 & 0.00 & 0.00 & 179 & 0.37 \\
\hline 0 & 0.89 & 0.89 & 0.89 & 22.90 & 22.90 & 19.12 & 87.80 & 87.80 & 0.00 & 0.00 & 179 & 0.49 \\
\hline 0 & 0.38 & 0.44 & 0.44 & 20.70 & 20.70 & 18.59 & 93.77 & 93.77 & 0.00 & 0.00 & 179 & 0.52 \\
\hline 0 & 1.17 & 0.91 & 0.91 & 24.00 & 24.00 & 20.46 & 87.12 & 87.12 & 0.00 & 0.00 & 179 & 0.49 \\
\hline 0 & 0.82 & 0.70 & 0.66 & 20.05 & 19.94 & 16.55 & 91.62 & 94.71 & 0.16 & 3.09 & 179 & 0.51 \\
\hline 0 & 0.78 & 0.75 & 0.62 & 27.60 & 27.47 & 25.76 & 64.96 & 73.33 & 0.31 & 8.37 & 179 & 0.36 \\
\hline 0 & 1.33 & 1.31 & 1.13 & 21.61 & 21.60 & 17.03 & 66.69 & 77.57 & 0.51 & 10.89 & 179 & 0.37 \\
\hline 0 & 1.24 & 1.07 & 1.07 & 23.09 & 23.09 & 18.09 & 96.04 & 96.04 & 0.00 & 0.00 & 179 & 0.54 \\
\hline 0 & 1.38 & 1.10 & 1.10 & 23.53 & 23.53 & 18.84 & 90.56 & 90.56 & 0.00 & 0.00 & 179 & 0.51 \\
\hline 0 & 0.80 & 0.79 & 0.79 & 25.19 & 25.19 & 22.39 & 83.81 & 83.81 & 0.00 & 0.00 & 179 & 0.47 \\
\hline 0 & 0.85 & 0.75 & 0.75 & 24.84 & 24.84 & 22.05 & 87.01 & 87.01 & 0.00 & 0.00 & 179 & 0.49 \\
\hline 0 & 0.84 & 0.78 & 0.78 & 23.02 & 23.02 & 20.46 & 70.36 & 70.36 & 0.00 & 0.00 & 179 & 0.39 \\
\hline 0 & 0.39 & 0.32 & 0.32 & 21.41 & 21.41 & 20.40 & 66.44 & 66.44 & 0.00 & 0.00 & 179 & 0.37 \\
\hline 0 & 0.38 & 0.36 & 0.27 & 24.02 & 23.78 & 22.72 & 84.44 & 90.57 & 0.25 & 6.13 & 179 & 0.47 \\
\hline 0 & 1.43 & 1.36 & 1.36 & 26.11 & 26.11 & 20.57 & 93.35 & 93.35 & 0.00 & 0.00 & 179 & 0.52 \\
\hline 0 & 1.46 & 1.25 & 1.25 & 26.86 & 26.86 & 23.00 & 76.31 & 76.31 & 0.00 & 0.00 & 179 & 0.43 \\
\hline 0 & 0.95 & 0.78 & 0.78 & 21.56 & 21.56 & 18.67 & 74.23 & 74.23 & 0.00 & 0.00 & 179 & 0.41 \\
\hline 0 & 0.71 & 0.64 & 0.64 & 21.25 & 21.25 & 18.45 & 86.42 & 86.42 & 0.00 & 0.00 & 179 & 0.48 \\
\hline
\end{tabular}




\begin{tabular}{|c|l|l|l|l|l|l|l|l|l|l|l|l|}
\hline 0 & 0.84 & 0.70 & 0.70 & 22.83 & 22.83 & 20.24 & 80.43 & 80.43 & 0.00 & 0.00 & 179 & 0.45 \\
\hline 0 & 0.66 & 0.63 & 0.63 & 22.29 & 22.29 & 19.51 & 91.50 & 91.50 & 0.00 & 0.00 & 179 & 0.51 \\
\hline 0 & 1.29 & 1.24 & 1.24 & 22.78 & 22.78 & 19.01 & 62.98 & 62.98 & 0.00 & 0.00 & 179 & 0.35 \\
\hline 0 & 1.06 & 0.84 & 0.84 & 24.95 & 24.95 & 21.77 & 88.22 & 88.22 & 0.00 & 0.00 & 179 & 0.49 \\
\hline 0 & 1.16 & 1.11 & 1.01 & 21.17 & 21.07 & 16.86 & 73.59 & 78.96 & 0.26 & 5.36 & 179 & 0.41 \\
\hline 0 & 2.32 & 2.14 & 2.14 & 26.79 & 26.79 & 20.52 & 68.37 & 68.37 & 0.00 & 0.00 & 179 & 0.38 \\
\hline 0 & 0.68 & 0.63 & 0.53 & 20.43 & 20.33 & 18.28 & 65.88 & 75.14 & 0.46 & 9.26 & 179 & 0.37 \\
\hline 0 & 0.89 & 0.86 & 0.86 & 24.14 & 24.14 & 21.24 & 75.60 & 75.60 & 0.00 & 0.00 & 179 & 0.42 \\
\hline 0 & 1.18 & 1.17 & 1.17 & 25.82 & 25.82 & 21.38 & 87.70 & 87.70 & 0.00 & 0.00 & 179 & 0.49 \\
\hline 0 & 0.90 & 0.86 & 0.86 & 22.39 & 22.39 & 18.34 & 95.53 & 95.53 & 0.00 & 0.00 & 179 & 0.53 \\
\hline 0 & 1.24 & 1.03 & 1.03 & 27.33 & 27.33 & 23.39 & 97.82 & 97.82 & 0.00 & 0.00 & 179 & 0.55 \\
\hline 0 & 1.23 & 1.14 & 1.14 & 23.45 & 23.45 & 18.12 & 96.83 & 96.83 & 0.00 & 0.00 & 179 & 0.54 \\
\hline 0 & 1.00 & 0.94 & 0.81 & 21.56 & 21.35 & 18.14 & 71.15 & 77.73 & 0.30 & 6.57 & 179 & 0.40 \\
\hline 0 & 0.76 & 0.76 & 0.76 & 21.11 & 21.11 & 17.67 & 87.15 & 87.15 & 0.00 & 0.00 & 179 & 0.49 \\
\hline 0 & 0.41 & 0.37 & 0.37 & 21.78 & 21.78 & 20.54 & 69.68 & 69.68 & 0.00 & 0.00 & 179 & 0.39 \\
\hline $\begin{array}{l}\text { Me } \\
\text { an }\end{array}$ & 0.94 & 0.91 & 0.89 & 23.29 & 23.28 & 20.16 & 74.41 & 76.06 & 0.07 & 1.65 & 179 & 0.42 \\
\hline Std & 0.38 & 0.36 & 0.36 & 2.35 & 2.34 & 2.46 & 14.82 & 15.15 & 0.17 & 4.03 & 0 & 0.08 \\
\hline
\end{tabular}


Table 4: Bronson Ave. (W-NS).

\begin{tabular}{|c|c|c|c|c|c|c|c|c|c|c|c|c|}
\hline $\begin{array}{l}\text { Tru } \\
\text { cks }\end{array}$ & $\begin{array}{c}\text { Mean } \\
\text { Decelerati } \\
\text { on }\end{array}$ & \begin{tabular}{|c|} 
Adjusted \\
Overall \\
Decelerati \\
on \\
\end{tabular} & \begin{tabular}{|c|} 
Original \\
Overall \\
Decelerati \\
on \\
\end{tabular} & $\begin{array}{l}\text { Initial } \\
\text { Speed }\end{array}$ & $\left|\begin{array}{c}\text { Diverg } \\
\mathrm{e} \\
\text { Speed }\end{array}\right|$ & $\begin{array}{c}\text { Final } \\
\text { Speed }\end{array}$ & $\begin{array}{l}\text { Decelera } \\
\text { tion } \\
\text { Distance }\end{array}$ & $\begin{array}{l}\text { Decelerati } \\
\text { on Length }\end{array}$ & $\begin{array}{l}\text { Decelerati } \\
\text { on Time }\end{array}$ & \begin{tabular}{|c} 
Distance \\
to \\
Decelera \\
te
\end{tabular} & $\begin{array}{c}\mathrm{SCL} \\
\text { Lengt } \\
\mathrm{h}\end{array}$ & $\begin{array}{l}\text { Utilizati } \\
\text { on Rate }\end{array}$ \\
\hline 0 & 1.15 & 1.07 & 1.07 & 23.05 & 23.05 & 19.37 & 72.64 & 72.64 & 0.00 & 0.00 & 212 & 0.34 \\
\hline 0 & 0.43 & 0.49 & 0.49 & 24.58 & 24.58 & 22.68 & 89.65 & 89.65 & 0.00 & 0.00 & 212 & 0.42 \\
\hline 0 & 0.43 & 1.89 & 1.89 & 23.05 & 23.05 & 18.31 & 50.86 & 50.86 & 0.00 & 0.00 & 212 & 0.24 \\
\hline 0 & 0.78 & 0.94 & 0.94 & 26.02 & 26.02 & 22.04 & 99.63 & 99.63 & 0.00 & 0.00 & 212 & 0.47 \\
\hline 0 & 1.41 & 1.30 & 1.30 & 22.63 & 22.63 & 14.13 & 119.16 & 119.16 & 0.00 & 0.00 & 212 & 0.56 \\
\hline 0 & 1.49 & 1.41 & 1.41 & 25.52 & 25.52 & 20.43 & 81.70 & 81.70 & 0.00 & 0.00 & 212 & 0.39 \\
\hline 0 & 1.13 & 1.10 & 0.69 & 25.53 & 25.80 & 22.43 & 66.31 & 117.95 & 2.03 & 51.64 & 212 & 0.31 \\
\hline 0 & 0.44 & 0.45 & 0.45 & 20.93 & 20.93 & 18.59 & 102.57 & 102.57 & 0.00 & 0.00 & 212 & 0.48 \\
\hline 0 & 0.44 & 1.19 & 1.19 & 24.05 & 24.05 & 19.40 & 83.70 & 83.70 & 0.00 & 0.00 & 212 & 0.39 \\
\hline 0 & 1.13 & 0.87 & 0.87 & 24.91 & 24.91 & 20.16 & 123.76 & 123.76 & 0.00 & 0.00 & 212 & 0.58 \\
\hline 0 & 1.13 & 1.43 & 1.43 & 23.88 & 23.88 & 18.65 & 76.05 & 76.05 & 0.00 & 0.00 & 212 & 0.36 \\
\hline 0 & 0.87 & 0.82 & 0.81 & 24.27 & 24.27 & 21.09 & 87.31 & 88.54 & 0.05 & 1.23 & 212 & 0.41 \\
\hline 0 & 1.46 & 1.16 & 1.16 & 24.80 & 24.80 & 20.54 & 83.25 & 83.25 & 0.00 & 0.00 & 212 & 0.39 \\
\hline 0 & 1.21 & 1.07 & 0.84 & 23.77 & 24.85 & 19.48 & 86.49 & 142.11 & 2.32 & 55.62 & 212 & 0.41 \\
\hline 0 & 1.46 & 1.24 & 1.24 & 25.71 & 25.71 & 19.70 & 109.22 & 109.22 & 0.00 & 0.00 & 212 & 0.52 \\
\hline 0 & 0.99 & 1.08 & 1.08 & 23.10 & 23.10 & 18.67 & 83.57 & 83.57 & 0.00 & 0.00 & 212 & 0.39 \\
\hline 0 & 0.99 & 1.08 & 1.08 & 25.47 & 25.47 & 21.21 & 90.74 & 90.74 & 0.00 & 0.00 & 212 & 0.43 \\
\hline 0 & 1.35 & 1.32 & 1.32 & 25.16 & 25.16 & 20.04 & 87.03 & 87.03 & 0.00 & 0.00 & 212 & 0.41 \\
\hline 0 & 1.35 & 1.38 & 1.38 & 26.03 & 26.03 & 21.51 & 76.87 & 76.87 & 0.00 & 0.00 & 212 & 0.36 \\
\hline 0 & 1.46 & 1.35 & 1.23 & 26.02 & 26.24 & 19.32 & 111.71 & 128.09 & 0.66 & 16.38 & 212 & 0.53 \\
\hline 0 & 1.26 & 0.83 & 0.83 & 23.38 & 23.38 & 20.04 & 89.15 & 89.15 & 0.00 & 0.00 & 212 & 0.42 \\
\hline 0 & 0.82 & 0.80 & 0.72 & 22.46 & 22.32 & 18.32 & 104.57 & 113.66 & 0.41 & 9.09 & 212 & 0.49 \\
\hline 0 & 1.39 & 1.28 & 1.28 & 24.39 & 24.39 & 20.09 & 74.34 & 74.34 & 0.00 & 0.00 & 212 & 0.35 \\
\hline 0 & 1.48 & 1.21 & 1.21 & 27.36 & 27.36 & 22.71 & 95.92 & 95.92 & 0.00 & 0.00 & 212 & 0.45 \\
\hline 0 & 0.80 & 0.73 & 0.67 & 23.35 & 23.43 & 19.59 & 110.70 & 123.61 & 0.56 & 12.91 & 212 & 0.52 \\
\hline 0 & 1.33 & 1.26 & 1.26 & 26.41 & 26.41 & 21.82 & 86.32 & 86.32 & 0.00 & 0.00 & 212 & 0.41 \\
\hline 0 & 0.75 & 0.69 & 0.65 & 22.93 & 22.99 & 19.40 & 108.44 & 116.20 & 0.31 & 7.77 & 212 & 0.51 \\
\hline 0 & 0.75 & 0.91 & 0.91 & 21.91 & 21.91 & 17.23 & 99.21 & 99.21 & 0.00 & 0.00 & 212 & 0.47 \\
\hline 0 & 1.33 & 0.80 & 0.80 & 25.71 & 25.71 & 21.82 & 117.65 & 117.65 & 0.00 & 0.00 & 212 & 0.55 \\
\hline 0 & 1.51 & 1.36 & 1.36 & 25.66 & 25.66 & 20.90 & 80.77 & 80.77 & 0.00 & 0.00 & 212 & 0.38 \\
\hline 0 & 1.91 & 1.79 & 1.79 & 30.50 & 30.50 & 24.38 & 92.50 & 92.50 & 0.00 & 0.00 & 212 & 0.44 \\
\hline 0 & 1.91 & 0.55 & 0.55 & 23.94 & 23.94 & 21.90 & 85.66 & 85.66 & 0.00 & 0.00 & 212 & 0.40 \\
\hline 0 & 0.77 & 0.79 & 0.79 & 20.82 & 20.82 & 14.61 & 137.57 & 137.57 & 0.00 & 0.00 & 212 & 0.65 \\
\hline 0 & 1.40 & 1.08 & 0.95 & 24.74 & 25.10 & 19.30 & 111.96 & 137.83 & 1.06 & 25.87 & 212 & 0.53 \\
\hline 0 & 1.37 & 1.12 & 0.98 & 26.05 & 26.41 & 20.29 & 118.16 & 145.45 & 1.06 & 27.30 & 212 & 0.56 \\
\hline
\end{tabular}




\begin{tabular}{|c|c|c|c|c|c|c|c|c|c|c|c|c|}
\hline 0 & 1.25 & 1.07 & 0.95 & 24.46 & 24.19 & 19.29 & 106.36 & 113.82 & 0.30 & 7.46 & 212 & 0.50 \\
\hline 0 & 1.28 & 0.95 & 0.74 & 23.88 & 23.35 & 19.38 & 103.07 & 116.40 & 0.56 & 13.33 & 212 & 0.49 \\
\hline 0 & 1.40 & 1.33 & 1.33 & 24.10 & 24.10 & 16.53 & 114.55 & 114.55 & 0.00 & 0.00 & 212 & 0.54 \\
\hline 0 & 1.60 & 1.39 & 1.39 & 26.66 & 26.66 & 20.90 & 98.51 & 98.51 & 0.00 & 0.00 & 212 & 0.46 \\
\hline 0 & 1.14 & 1.07 & 1.07 & 25.32 & 25.32 & 19.65 & 119.32 & 119.32 & 0.00 & 0.00 & 212 & 0.56 \\
\hline 0 & 1.76 & 1.76 & 1.26 & 24.83 & 26.35 & 17.95 & 83.68 & 150.43 & 2.63 & 66.74 & 212 & 0.39 \\
\hline 0 & 1.39 & 0.83 & 0.83 & 21.63 & 21.63 & 16.09 & 132.12 & 132.12 & 0.00 & 0.00 & 212 & 0.62 \\
\hline 0 & 0.59 & 0.58 & 0.58 & 22.66 & 22.66 & 19.65 & 109.31 & 109.31 & 0.00 & 0.00 & 212 & 0.52 \\
\hline 0 & 1.06 & 1.10 & 1.10 & 25.02 & 25.02 & 19.12 & 116.85 & 116.85 & 0.00 & 0.00 & 212 & 0.55 \\
\hline 0 & 0.82 & 0.72 & 0.62 & 22.32 & 22.57 & 18.21 & 116.78 & 144.53 & 1.26 & 27.74 & 212 & 0.55 \\
\hline 0 & 1.12 & 0.95 & 0.95 & 27.02 & 27.02 & 22.40 & 120.52 & 120.52 & 0.00 & 0.00 & 212 & 0.57 \\
\hline 0 & 1.17 & 1.13 & 1.01 & 23.66 & 25.13 & 19.59 & 76.82 & 121.46 & 1.86 & 44.64 & 212 & 0.36 \\
\hline 0 & 1.17 & 0.50 & 0.50 & 21.79 & 21.79 & 18.37 & 138.60 & 138.60 & 0.00 & 0.00 & 212 & 0.65 \\
\hline 0 & 1.01 & 0.89 & 0.89 & 25.55 & 25.55 & 22.76 & 75.24 & 75.24 & 0.00 & 0.00 & 212 & 0.35 \\
\hline 0 & 0.90 & 0.80 & 0.80 & 21.80 & 21.80 & 17.01 & 117.06 & 117.06 & 0.00 & 0.00 & 212 & 0.55 \\
\hline 0 & 2.20 & 1.88 & 1.88 & 28.47 & 28.47 & 21.02 & 99.47 & 99.47 & 0.00 & 0.00 & 212 & 0.47 \\
\hline 0 & 1.80 & 1.76 & 1.76 & 27.60 & 27.60 & 19.15 & 109.90 & 109.90 & 0.00 & 0.00 & 212 & 0.52 \\
\hline 0 & 1.52 & 1.38 & 1.38 & 28.13 & 28.13 & 21.07 & 125.21 & 125.21 & 0.00 & 0.00 & 212 & 0.59 \\
\hline 0 & 0.89 & 0.84 & 0.84 & 23.13 & 23.13 & 16.90 & 148.13 & 148.13 & 0.00 & 0.00 & 212 & 0.70 \\
\hline 0 & 0.84 & 0.80 & 0.80 & 22.77 & 22.77 & 18.32 & 113.62 & 113.62 & 0.00 & 0.00 & 212 & 0.54 \\
\hline 0 & 0.92 & 0.63 & 0.63 & 18.57 & 18.57 & 15.28 & 89.16 & 89.16 & 0.00 & 0.00 & 212 & 0.42 \\
\hline 0 & 0.77 & 0.63 & 0.54 & 26.30 & 26.13 & 23.04 & 127.67 & 140.98 & 0.51 & 13.31 & 212 & 0.60 \\
\hline 0 & 0.88 & 0.89 & 0.89 & 21.94 & 21.94 & 18.12 & 85.03 & 85.03 & 0.00 & 0.00 & 212 & 0.40 \\
\hline 0 & 0.68 & 0.65 & 0.65 & 22.91 & 22.91 & 19.95 & 96.72 & 96.72 & 0.00 & 0.00 & 212 & 0.46 \\
\hline 0 & 1.30 & 1.24 & 1.24 & 24.77 & 24.77 & 17.96 & 115.62 & 115.62 & 0.00 & 0.00 & 212 & 0.55 \\
\hline 0 & 2.38 & 2.16 & 2.16 & 27.05 & 27.05 & 18.12 & 93.13 & 93.13 & 0.00 & 0.00 & 212 & 0.44 \\
\hline 0 & 1.45 & 1.24 & 1.03 & 19.18 & 18.71 & 12.61 & 84.25 & 94.13 & 0.50 & 9.88 & 212 & 0.40 \\
\hline 0 & 0.67 & 0.66 & 0.70 & 22.58 & 24.49 & 19.87 & 85.96 & 143.42 & 2.46 & 57.45 & 212 & 0.41 \\
\hline 0 & 1.03 & 1.06 & 1.06 & 21.52 & 21.52 & 17.67 & 70.61 & 70.61 & 0.00 & 0.00 & 212 & 0.33 \\
\hline 0 & 1.64 & 1.20 & 1.17 & 24.27 & 24.27 & 17.70 & 115.98 & 119.65 & 0.15 & 3.67 & 212 & 0.55 \\
\hline 0 & 1.64 & 0.82 & 0.82 & 25.60 & 25.60 & 20.49 & 144.44 & 144.44 & 0.00 & 0.00 & 212 & 0.68 \\
\hline 0 & 1.61 & 1.35 & 1.35 & 28.66 & 28.66 & 23.40 & 100.94 & 100.94 & 0.00 & 0.00 & 212 & 0.48 \\
\hline 0 & 1.03 & 0.86 & 0.86 & 26.10 & 26.10 & 22.29 & 106.58 & 106.58 & 0.00 & 0.00 & 212 & 0.50 \\
\hline 0 & 1.11 & 1.04 & 1.04 & 22.63 & 22.63 & 14.45 & 145.59 & 145.59 & 0.00 & 0.00 & 212 & 0.69 \\
\hline 0 & 0.81 & 0.74 & 0.05 & 23.08 & 20.99 & 20.73 & 68.84 & 110.13 & 1.72 & 41.29 & 212 & 0.32 \\
\hline 0 & 1.66 & 1.48 & 1.48 & 27.50 & 27.50 & 22.63 & 81.79 & 81.79 & 0.00 & 0.00 & 212 & 0.39 \\
\hline 0 & 1.66 & 2.14 & 2.14 & 29.61 & 29.61 & 23.04 & 79.36 & 79.36 & 0.00 & 0.00 & 212 & 0.37 \\
\hline 0 & 1.66 & 0.66 & 0.66 & 22.24 & 22.24 & 19.23 & 99.12 & 99.12 & 0.00 & 0.00 & 212 & 0.47 \\
\hline 0 & 1.66 & 0.67 & 0.67 & 23.10 & 23.10 & 20.04 & 97.94 & 97.94 & 0.00 & 0.00 & 212 & 0.46 \\
\hline 0 & 0.75 & 0.60 & 0.60 & 19.99 & 19.99 & 15.25 & 138.80 & 138.80 & 0.00 & 0.00 & 212 & 0.65 \\
\hline
\end{tabular}




\begin{tabular}{|c|c|c|c|c|c|c|c|c|c|c|c|c|}
\hline 0 & 0.45 & 0.40 & 0.40 & 22.74 & 22.74 & 20.84 & 104.39 & 104.39 & 0.00 & 0.00 & 212 & 0.49 \\
\hline 0 & 0.77 & 0.76 & 0.64 & 23.44 & 24.41 & 20.65 & 80.77 & 132.17 & 2.18 & 51.40 & 212 & 0.38 \\
\hline 0 & 0.77 & 0.58 & 0.58 & 20.46 & 20.46 & 17.73 & 91.89 & 91.89 & 0.00 & 0.00 & 212 & 0.43 \\
\hline 0 & 0.67 & 0.64 & 0.20 & 23.49 & 21.99 & 21.09 & 82.58 & 98.05 & 0.65 & 15.47 & 212 & 0.39 \\
\hline 0 & 0.93 & 1.01 & 1.01 & 25.43 & 25.43 & 19.87 & 123.25 & 123.25 & 0.00 & 0.00 & 212 & 0.58 \\
\hline 0 & 0.95 & 0.83 & 0.69 & 21.80 & 22.18 & 18.48 & 80.85 & 109.49 & 1.26 & 28.64 & 212 & 0.38 \\
\hline 0 & 0.60 & 0.53 & 0.53 & 23.68 & 23.68 & 20.73 & 122.79 & 122.79 & 0.00 & 0.00 & 212 & 0.58 \\
\hline 0 & 1.68 & 1.42 & 1.42 & 27.16 & 27.16 & 20.54 & 110.69 & 110.69 & 0.00 & 0.00 & 212 & 0.52 \\
\hline 0 & 1.51 & 1.41 & 1.25 & 22.97 & 23.55 & 15.59 & 100.40 & 125.83 & 1.11 & 25.43 & 212 & 0.47 \\
\hline 0 & 1.51 & 1.64 & 1.64 & 24.10 & 24.10 & 15.11 & 106.12 & 106.12 & 0.00 & 0.00 & 212 & 0.50 \\
\hline 1 & 1.20 & 1.14 & 0.79 & 25.91 & 25.30 & 22.29 & 75.96 & 90.47 & 0.57 & 14.50 & 212 & 0.36 \\
\hline 1 & 1.06 & 1.02 & 1.02 & 21.21 & 21.21 & 14.08 & 122.06 & 122.06 & 0.00 & 0.00 & 212 & 0.58 \\
\hline 1 & 1.76 & 1.75 & 1.28 & 24.38 & 24.88 & 16.67 & 89.62 & 135.51 & 1.87 & 45.89 & 212 & 0.42 \\
\hline 1 & 1.10 & 1.03 & 0.92 & 22.43 & 22.38 & 16.86 & 105.13 & 117.55 & 0.56 & 12.42 & 212 & 0.50 \\
\hline 1 & 1.93 & 1.86 & 1.69 & 21.18 & 22.93 & 6.73 & 105.96 & 142.32 & 1.71 & 36.36 & 212 & 0.50 \\
\hline $\begin{array}{l}\text { Me } \\
\text { an }\end{array}$ & 1.19 & 1.07 & 1.01 & 24.19 & 24.25 & 19.25 & 100.56 & 108.60 & 0.34 & 8.04 & 212 & 0.47 \\
\hline Std & 0.42 & 0.39 & 0.40 & 2.25 & 2.28 & 2.72 & 19.96 & 22.11 & 0.67 & 16.01 & 0 & 0.09 \\
\hline
\end{tabular}


Table 5: Moodie Drive (W-NS).

\begin{tabular}{|c|c|c|c|c|c|c|c|c|c|c|c|c|}
\hline $\begin{array}{l}\text { Tru } \\
\text { cks }\end{array}$ & $\begin{array}{l}\text { Mean } \\
\text { Decele } \\
\text { ration }\end{array}$ & $\begin{array}{c}\text { Adjusted } \\
\text { Overall } \\
\text { Deceleration }\end{array}$ & $\begin{array}{c}\text { Original } \\
\text { Overall } \\
\text { Deceleration }\end{array}$ & $\begin{array}{l}\text { Initial } \\
\text { Speed }\end{array}$ & $\left|\begin{array}{c}\text { Diverg } \\
\mathrm{e} \\
\text { Speed }\end{array}\right|$ & $\begin{array}{c}\text { Final } \\
\text { Speed }\end{array}$ & $\begin{array}{c}\text { Decelera } \\
\text { tion } \\
\text { Distance }\end{array}$ & $\begin{array}{l}\text { Decelerati } \\
\text { on Length }\end{array}$ & $\begin{array}{l}\text { Decelerati } \\
\text { on Time }\end{array}$ & \begin{tabular}{|c} 
Distance \\
to \\
Decelera \\
te \\
\end{tabular} & $\begin{array}{c}\mathrm{SCL} \\
\text { Lengt } \\
\mathrm{h}\end{array}$ & $\begin{array}{l}\text { Utilizati } \\
\text { on Rate }\end{array}$ \\
\hline 0 & 0.58 & 0.52 & 0.27 & 28.59 & 28.67 & 27.06 & 81.87 & 167.09 & 2.97 & 85.23 & 292 & 0.28 \\
\hline 0 & 0.38 & 0.36 & 0.36 & 31.20 & 31.20 & 29.72 & 126.04 & 126.04 & 0.00 & 0.00 & 292 & 0.43 \\
\hline 0 & 0.33 & 0.16 & 0.16 & 27.72 & 27.72 & 26.92 & 135.30 & 135.30 & 0.00 & 0.00 & 292 & 0.46 \\
\hline 0 & 0.17 & 0.23 & 0.23 & 28.56 & 28.56 & 27.59 & 119.25 & 119.25 & 0.00 & 0.00 & 292 & 0.41 \\
\hline 0 & 0.62 & 0.58 & 0.58 & 26.72 & 26.72 & 23.36 & 147.09 & 147.09 & 0.00 & 0.00 & 292 & 0.50 \\
\hline 0 & 0.44 & 0.39 & 0.39 & 28.39 & 28.39 & 26.53 & 129.89 & 129.89 & 0.00 & 0.00 & 292 & 0.44 \\
\hline 0 & 0.46 & 0.46 & 0.46 & 29.59 & 29.59 & 26.86 & 165.65 & 165.65 & 0.00 & 0.00 & 292 & 0.57 \\
\hline 0 & 0.63 & 0.54 & 0.32 & 28.97 & 28.95 & 27.39 & 82.04 & 137.57 & 1.91 & 55.53 & 292 & 0.28 \\
\hline 0 & 0.15 & 0.10 & 0.10 & 27.03 & 27.03 & 26.53 & 138.81 & 138.81 & 0.00 & 0.00 & 292 & 0.48 \\
\hline 0 & 0.47 & 0.44 & 0.28 & 28.14 & 28.45 & 26.34 & 111.90 & 207.43 & 3.37 & 95.53 & 292 & 0.38 \\
\hline 0 & 0.45 & 0.46 & 0.46 & 31.06 & 31.06 & 29.36 & 111.05 & 111.05 & 0.00 & 0.00 & 292 & 0.38 \\
\hline 0 & 0.25 & 0.18 & 0.10 & 21.72 & 21.42 & 20.67 & 121.60 & 153.07 & 1.40 & 31.47 & 292 & 0.42 \\
\hline 0 & 0.34 & 0.34 & 0.22 & 29.17 & 28.92 & 27.59 & 130.11 & 170.98 & 1.42 & 40.87 & 292 & 0.45 \\
\hline 0 & 0.08 & 0.07 & 0.07 & 24.20 & 24.20 & 23.58 & 225.57 & 225.57 & 0.00 & 0.00 & 292 & 0.77 \\
\hline 0 & 0.52 & 0.50 & 0.36 & 28.61 & 28.61 & 26.78 & 101.77 & 142.81 & 1.45 & 41.04 & 292 & 0.35 \\
\hline 0 & 0.58 & 0.56 & 0.31 & 31.03 & 30.78 & 29.53 & 80.86 & 121.41 & 1.31 & 40.55 & 292 & 0.28 \\
\hline 0 & 0.68 & 0.51 & 0.40 & 27.72 & 28.20 & 25.39 & 121.82 & 187.50 & 2.37 & 65.68 & 292 & 0.42 \\
\hline 0 & 0.40 & 0.27 & -0.12 & 31.00 & 29.08 & 29.89 & 123.90 & 199.07 & 2.47 & 75.16 & 292 & 0.42 \\
\hline 0 & 0.06 & 0.03 & 0.03 & 27.78 & 27.78 & 27.59 & 200.65 & 200.65 & 0.00 & 0.00 & 292 & 0.69 \\
\hline 0 & 0.27 & 0.27 & 0.27 & 29.89 & 29.89 & 28.39 & 162.15 & 162.15 & 0.00 & 0.00 & 292 & 0.56 \\
\hline 0 & 0.56 & 0.48 & 0.35 & 31.61 & 31.64 & 29.71 & 121.62 & 168.79 & 1.42 & 47.17 & 292 & 0.42 \\
\hline 0 & 0.39 & 0.39 & 0.22 & 28.34 & 28.31 & 27.00 & 93.42 & 164.64 & 2.52 & 71.22 & 292 & 0.32 \\
\hline 0 & 0.50 & 0.32 & 0.26 & 29.84 & 29.64 & 28.00 & 165.87 & 182.26 & 0.50 & 16.39 & 292 & 0.57 \\
\hline 0 & 0.67 & 0.65 & 0.50 & 28.03 & 29.22 & 25.53 & 102.14 & 204.41 & 3.53 & 102.27 & 292 & 0.35 \\
\hline 0 & 0.17 & 0.09 & 0.09 & 30.89 & 30.89 & 30.38 & 166.53 & 166.53 & 0.00 & 0.00 & 292 & 0.57 \\
\hline 0 & 0.17 & 0.08 & 0.08 & 22.95 & 22.95 & 22.39 & 151.96 & 151.96 & 0.00 & 0.00 & 292 & 0.52 \\
\hline 0 & 0.54 & 0.48 & 0.48 & 28.86 & 28.86 & 26.01 & 162.02 & 162.02 & 0.00 & 0.00 & 292 & 0.55 \\
\hline 0 & 0.85 & 0.78 & 0.71 & 33.11 & 33.17 & 30.33 & 112.91 & 126.24 & 0.41 & 13.33 & 292 & 0.39 \\
\hline 0 & 0.33 & 0.32 & 0.32 & 25.36 & 25.36 & 22.86 & 188.16 & 188.16 & 0.00 & 0.00 & 292 & 0.64 \\
\hline 0 & 0.61 & 0.57 & 0.46 & 33.31 & 33.28 & 31.25 & 117.11 & 142.25 & 0.76 & 25.14 & 292 & 0.40 \\
\hline 0 & 0.57 & 0.52 & 0.46 & 27.59 & 27.64 & 25.13 & 124.06 & 143.27 & 0.71 & 19.21 & 292 & 0.42 \\
\hline 0 & 0.47 & 0.48 & 0.36 & 27.86 & 28.56 & 26.27 & 89.38 & 175.83 & 3.02 & 86.46 & 292 & 0.31 \\
\hline 0 & 0.22 & 0.21 & 0.21 & 28.89 & 28.89 & 27.67 & 162.37 & 162.37 & 0.00 & 0.00 & 292 & 0.56 \\
\hline 0 & 0.16 & 0.14 & 0.14 & 26.08 & 26.08 & 25.17 & 173.81 & 173.81 & 0.00 & 0.00 & 292 & 0.60 \\
\hline 0 & 0.16 & 0.56 & 0.56 & 27.28 & 27.28 & 26.13 & 54.53 & 54.53 & 0.00 & 0.00 & 292 & 0.19 \\
\hline
\end{tabular}




\begin{tabular}{|c|c|c|c|c|c|c|c|c|c|c|c|c|}
\hline 0 & 0.49 & 0.34 & 0.15 & 28.70 & 28.20 & 27.21 & 123.31 & 182.00 & 2.07 & 58.69 & 292 & 0.42 \\
\hline 0 & 0.59 & 0.55 & 0.44 & 25.50 & 26.58 & 23.14 & 102.75 & 195.33 & 3.58 & 92.58 & 292 & 0.35 \\
\hline 0 & 0.25 & 0.17 & 0.17 & 30.84 & 30.84 & 30.00 & 145.21 & 145.21 & 0.00 & 0.00 & 292 & 0.50 \\
\hline 0 & 0.30 & 0.22 & 0.22 & 27.11 & 27.11 & 25.75 & 161.18 & 161.18 & 0.00 & 0.00 & 292 & 0.55 \\
\hline 0 & 0.30 & 0.45 & 0.45 & 27.09 & 27.09 & 24.61 & 140.45 & 140.45 & 0.00 & 0.00 & 292 & 0.48 \\
\hline 0 & 0.28 & 0.26 & 0.26 & 22.45 & 22.45 & 21.45 & 82.50 & 82.50 & 0.00 & 0.00 & 292 & 0.28 \\
\hline 0 & 0.44 & 0.40 & 0.36 & 24.64 & 25.39 & 22.22 & 142.08 & 209.67 & 2.73 & 67.58 & 292 & 0.49 \\
\hline 0 & 0.26 & 0.23 & 0.08 & 25.78 & 25.47 & 24.81 & 106.17 & 207.24 & 3.93 & 101.07 & 292 & 0.36 \\
\hline 0 & 0.43 & 0.55 & -0.31 & 33.39 & 30.03 & 31.98 & 84.29 & 194.43 & 3.48 & 110.14 & 292 & 0.29 \\
\hline 0 & 0.33 & 0.26 & 0.20 & 28.92 & 28.78 & 27.56 & 147.22 & 174.88 & 0.97 & 27.66 & 292 & 0.50 \\
\hline 0 & 0.17 & 0.12 & 0.12 & 30.34 & 30.34 & 29.70 & 153.10 & 153.10 & 0.00 & 0.00 & 292 & 0.52 \\
\hline 0 & 0.40 & 0.28 & 0.28 & 29.61 & 29.61 & 28.13 & 153.13 & 153.13 & 0.00 & 0.00 & 292 & 0.52 \\
\hline 0 & 0.46 & 0.24 & 0.24 & 26.50 & 26.50 & 24.64 & 198.49 & 198.49 & 0.00 & 0.00 & 292 & 0.68 \\
\hline 0 & 0.23 & 0.16 & 0.16 & 31.09 & 31.09 & 30.31 & 152.94 & 152.94 & 0.00 & 0.00 & 292 & 0.52 \\
\hline 0 & 0.44 & 0.36 & 0.36 & 31.06 & 31.06 & 28.84 & 185.33 & 185.33 & 0.00 & 0.00 & 292 & 0.63 \\
\hline 0 & 0.61 & 0.49 & 0.40 & 28.22 & 28.20 & 25.40 & 155.26 & 188.01 & 1.17 & 32.75 & 292 & 0.53 \\
\hline 0 & 0.25 & 0.26 & 0.26 & 26.89 & 26.89 & 25.31 & 157.71 & 157.71 & 0.00 & 0.00 & 292 & 0.54 \\
\hline 0 & 0.46 & 0.46 & 0.46 & 29.75 & 29.75 & 26.73 & 183.98 & 183.98 & 0.00 & 0.00 & 292 & 0.63 \\
\hline 0 & 0.72 & 0.66 & 0.40 & 28.25 & 28.67 & 25.95 & 94.38 & 185.89 & 3.23 & 91.52 & 292 & 0.32 \\
\hline 0 & 0.43 & 0.38 & 0.20 & 30.56 & 30.25 & 29.25 & 101.53 & 146.04 & 1.47 & 44.51 & 292 & 0.35 \\
\hline 0 & 0.39 & 0.37 & 0.22 & 25.36 & 25.50 & 23.78 & 105.35 & 189.22 & 3.32 & 83.87 & 292 & 0.36 \\
\hline 0 & 0.30 & 0.23 & 0.23 & 27.03 & 27.03 & 26.03 & 116.16 & 116.16 & 0.00 & 0.00 & 292 & 0.40 \\
\hline 0 & 0.36 & 0.33 & 0.33 & 26.78 & 26.78 & 26.23 & 43.75 & 43.75 & 0.00 & 0.00 & 292 & 0.15 \\
\hline 0 & 0.36 & 0.23 & 0.23 & 30.42 & 30.42 & 29.92 & 66.40 & 66.40 & 0.00 & 0.00 & 292 & 0.23 \\
\hline 0 & 0.51 & 0.45 & 0.38 & 30.95 & 31.20 & 29.14 & 119.04 & 161.36 & 1.37 & 42.32 & 292 & 0.41 \\
\hline 0 & 0.01 & 0.06 & 0.06 & 27.70 & 27.70 & 27.23 & 207.45 & 207.45 & 0.00 & 0.00 & 292 & 0.71 \\
\hline 0 & 0.17 & 0.23 & 0.23 & 28.28 & 28.28 & 26.50 & 213.80 & 213.80 & 0.00 & 0.00 & 292 & 0.73 \\
\hline 0 & 0.84 & 0.56 & 0.55 & 31.70 & 31.81 & 28.34 & 180.62 & 192.08 & 0.31 & 11.45 & 292 & 0.62 \\
\hline 0 & 0.42 & 0.35 & 0.35 & 30.39 & 30.39 & 28.42 & 163.96 & 163.96 & 0.00 & 0.00 & 292 & 0.56 \\
\hline 0 & 0.70 & 0.68 & 0.33 & 29.31 & 28.92 & 26.78 & 104.64 & 183.13 & 2.67 & 78.50 & 292 & 0.36 \\
\hline 0 & 0.58 & 0.53 & 0.44 & 30.00 & 30.36 & 27.60 & 128.67 & 183.12 & 1.81 & 54.45 & 292 & 0.44 \\
\hline 0 & 0.45 & 0.50 & 0.30 & 28.34 & 28.25 & 26.45 & 103.15 & 163.83 & 2.07 & 60.68 & 292 & 0.35 \\
\hline 0 & 0.31 & 0.28 & 0.28 & 25.47 & 25.47 & 23.81 & 148.30 & 148.30 & 0.00 & 0.00 & 292 & 0.51 \\
\hline 0 & 0.16 & 0.08 & 0.08 & 27.20 & 27.20 & 26.84 & 120.84 & 120.84 & 0.00 & 0.00 & 292 & 0.41 \\
\hline 0 & 0.38 & 0.35 & 0.35 & 27.08 & 27.08 & 24.43 & 197.59 & 197.59 & 0.00 & 0.00 & 292 & 0.68 \\
\hline 0 & 0.49 & 0.45 & 0.45 & 26.42 & 26.42 & 22.75 & 198.76 & 198.76 & 0.00 & 0.00 & 292 & 0.68 \\
\hline 0 & 0.60 & 0.59 & 0.59 & 32.56 & 32.56 & 29.60 & 155.12 & 155.12 & 0.00 & 0.00 & 292 & 0.53 \\
\hline 0 & 0.68 & 0.64 & 0.57 & 26.03 & 26.78 & 23.17 & 109.08 & 159.30 & 1.91 & 50.22 & 292 & 0.37 \\
\hline 0 & 0.52 & 0.37 & 0.37 & 30.61 & 30.61 & 28.70 & 152.30 & 152.30 & 0.00 & 0.00 & 292 & 0.52 \\
\hline 0 & 0.96 & 0.76 & 0.68 & 33.47 & 33.67 & 29.17 & 176.62 & 211.06 & 0.96 & 34.43 & 292 & 0.60 \\
\hline
\end{tabular}




\begin{tabular}{|c|c|c|c|c|c|c|c|c|c|c|c|c|}
\hline 0 & 0.41 & 0.28 & 0.24 & 30.67 & 30.84 & 29.28 & 147.74 & 195.36 & 1.56 & 47.61 & 292 & 0.51 \\
\hline 0 & 0.56 & 0.36 & 0.13 & 30.50 & 29.75 & 28.89 & 134.66 & 194.93 & 2.02 & 60.26 & 292 & 0.46 \\
\hline 0 & 0.31 & 0.40 & 0.40 & 31.61 & 31.61 & 29.56 & 156.56 & 156.56 & 0.00 & 0.00 & 292 & 0.54 \\
\hline 1 & 0.30 & 0.20 & 0.07 & 27.75 & 27.11 & 26.49 & 168.14 & 243.59 & 2.76 & 75.44 & 292 & 0.58 \\
\hline 1 & 0.61 & 0.55 & 0.55 & 24.03 & 24.03 & 21.45 & 106.28 & 106.28 & 0.00 & 0.00 & 292 & 0.36 \\
\hline 1 & 0.55 & 0.49 & 0.49 & 25.20 & 25.20 & 22.17 & 145.32 & 145.32 & 0.00 & 0.00 & 292 & 0.50 \\
\hline 1 & 0.29 & 0.21 & 0.21 & 22.20 & 22.20 & 21.22 & 100.35 & 100.35 & 0.00 & 0.00 & 292 & 0.34 \\
\hline 1 & 0.46 & 0.25 & 0.25 & 25.95 & 25.95 & 23.86 & 214.93 & 214.93 & 0.00 & 0.00 & 292 & 0.74 \\
\hline 1 & 0.66 & 0.55 & 0.55 & 29.56 & 29.56 & 25.86 & 184.81 & 184.81 & 0.00 & 0.00 & 292 & 0.63 \\
\hline 1 & 0.20 & 0.14 & 0.14 & 26.33 & 26.33 & 25.19 & 217.95 & 217.95 & 0.00 & 0.00 & 292 & 0.75 \\
\hline $\begin{array}{c}\text { Me } \\
\text { an }\end{array}$ & 0.42 & 0.37 & 0.30 & 28.38 & 28.36 & 26.62 & 138.38 & 163.54 & 0.88 & 25.15 & 292 & 0.47 \\
\hline Std & 0.19 & 0.17 & 0.17 & 2.57 & 2.49 & 2.56 & 39.26 & 37.44 & 1.21 & 33.90 & 0 & 0.13 \\
\hline
\end{tabular}


Table 6: Terry Fox Drive (E-NS).

\begin{tabular}{|c|c|c|c|c|c|c|c|c|c|c|c|c|}
\hline $\begin{array}{l}\text { Tru } \\
\text { cks }\end{array}$ & $\begin{array}{c}\text { Mean } \\
\text { Decelera } \\
\text { tion }\end{array}$ & \begin{tabular}{|c|} 
Adjusted \\
Overall \\
Decelerati \\
on \\
\end{tabular} & \begin{tabular}{|c|} 
Original \\
Overall \\
Decelerati \\
on \\
\end{tabular} & $\begin{array}{l}\text { Initial } \\
\text { Speed }\end{array}$ & $\begin{array}{c}\text { Diverg } \\
\text { e } \\
\text { Speed }\end{array}$ & $\begin{array}{c}\text { Final } \\
\text { Speed }\end{array}$ & $\begin{array}{l}\text { Decelera } \\
\text { tion } \\
\text { Distance }\end{array}$ & $\begin{array}{l}\text { Decelerati } \\
\text { on Length }\end{array}$ & $\begin{array}{l}\text { Decelerati } \\
\text { on Time }\end{array}$ & $\begin{array}{c}\text { Distance } \\
\text { to } \\
\text { Decelera } \\
\text { te }\end{array}$ & $\begin{array}{c}\mathrm{SCL} \\
\text { Lengt } \\
\mathrm{h}\end{array}$ & $\begin{array}{l}\text { Utilizati } \\
\text { on Rate }\end{array}$ \\
\hline 0 & 0.16 & 0.19 & 0.19 & 28.42 & 28.42 & 26.64 & 260.93 & 260.93 & 0.00 & 0.00 & 388 & 0.67 \\
\hline 0 & 0.59 & 0.60 & 0.60 & 32.99 & 32.99 & 29.03 & 202.87 & 202.87 & 0.00 & 0.00 & 388 & 0.52 \\
\hline 0 & 0.27 & 0.32 & 0.32 & 27.38 & 27.38 & 23.84 & 279.55 & 279.55 & 0.00 & 0.00 & 388 & 0.72 \\
\hline 0 & 0.57 & 0.53 & 0.53 & 31.01 & 31.01 & 28.17 & 159.09 & 159.09 & 0.00 & 0.00 & 388 & 0.41 \\
\hline 0 & 0.54 & 0.50 & 0.50 & 28.86 & 28.86 & 24.48 & 226.86 & 226.86 & 0.00 & 0.00 & 388 & 0.58 \\
\hline 0 & 0.27 & 0.30 & 0.30 & 29.84 & 29.84 & 27.25 & 250.18 & 250.18 & 0.00 & 0.00 & 388 & 0.64 \\
\hline 0 & 0.48 & 0.38 & 0.38 & 28.66 & 28.66 & 25.73 & 209.47 & 209.47 & 0.00 & 0.00 & 388 & 0.54 \\
\hline 0 & 0.45 & 0.25 & 0.12 & 27.59 & 27.62 & 26.39 & 131.09 & 270.40 & 5.04 & 139.30 & 388 & 0.34 \\
\hline 0 & 0.33 & 0.24 & 0.18 & 29.76 & 29.60 & 28.56 & 144.54 & 170.04 & 0.87 & 25.50 & 388 & 0.37 \\
\hline 0 & 0.36 & 0.31 & 0.31 & 28.58 & 28.58 & 25.20 & 290.08 & 290.08 & 0.00 & 0.00 & 388 & 0.75 \\
\hline 0 & 0.43 & 0.45 & 0.45 & 25.38 & 25.38 & 19.67 & 285.99 & 285.99 & 0.00 & 0.00 & 388 & 0.74 \\
\hline 0 & 0.30 & 0.19 & 0.18 & 29.90 & 30.20 & 28.74 & 174.35 & 239.40 & 2.17 & 65.05 & 388 & 0.45 \\
\hline 0 & 0.39 & 0.27 & 0.21 & 31.41 & 31.38 & 29.53 & 212.19 & 272.70 & 1.92 & 60.51 & 388 & 0.55 \\
\hline 0 & 0.18 & 0.21 & 0.21 & 23.67 & 23.67 & 20.50 & 324.75 & 324.75 & 0.00 & 0.00 & 388 & 0.84 \\
\hline 0 & 0.14 & 0.23 & 0.23 & 30.22 & 30.22 & 28.01 & 282.52 & 282.52 & 0.00 & 0.00 & 388 & 0.73 \\
\hline 0 & 0.61 & 0.60 & 0.60 & 29.66 & 29.66 & 24.62 & 225.46 & 225.46 & 0.00 & 0.00 & 388 & 0.58 \\
\hline 0 & 0.41 & 0.36 & 0.36 & 30.26 & 30.26 & 26.92 & 261.36 & 261.36 & 0.00 & 0.00 & 388 & 0.67 \\
\hline 0 & 0.45 & 0.51 & 0.51 & 28.35 & 28.35 & 25.34 & 158.18 & 158.18 & 0.00 & 0.00 & 388 & 0.41 \\
\hline 0 & 0.57 & 0.31 & 0.31 & 28.75 & 28.75 & 26.12 & 234.87 & 234.87 & 0.00 & 0.00 & 388 & 0.61 \\
\hline 0 & 0.64 & 0.60 & 0.03 & 30.34 & 28.67 & 28.33 & 98.01 & 317.98 & 7.41 & 219.96 & 388 & 0.25 \\
\hline 0 & 0.22 & 0.36 & 0.36 & 29.13 & 29.13 & 26.75 & 180.71 & 180.71 & 0.00 & 0.00 & 388 & 0.47 \\
\hline 0 & 0.85 & 0.74 & 0.43 & 30.51 & 31.77 & 27.78 & 107.08 & 281.10 & 5.60 & 174.03 & 388 & 0.28 \\
\hline 0 & 0.15 & 0.11 & 0.01 & 27.74 & 27.02 & 26.94 & 192.62 & 220.64 & 1.00 & 28.02 & 388 & 0.50 \\
\hline 0 & 0.17 & 0.27 & 0.57 & 25.17 & 27.71 & 23.94 & 109.18 & 166.52 & 2.16 & 57.34 & 388 & 0.28 \\
\hline 0 & 0.20 & 0.25 & 0.32 & 25.29 & 27.43 & 23.95 & 132.12 & 279.28 & 5.59 & 147.16 & 388 & 0.34 \\
\hline 0 & 0.20 & 0.26 & 0.26 & 29.52 & 29.52 & 27.50 & 222.81 & 222.81 & 0.00 & 0.00 & 388 & 0.57 \\
\hline 0 & 0.54 & 0.51 & 0.22 & 33.71 & 32.80 & 30.76 & 188.11 & 305.02 & 3.48 & 116.91 & 388 & 0.48 \\
\hline 0 & 0.18 & 0.28 & 0.28 & 26.22 & 26.22 & 23.50 & 244.19 & 244.19 & 0.00 & 0.00 & 388 & 0.63 \\
\hline 0 & 0.91 & 0.72 & 0.51 & 31.12 & 31.72 & 27.75 & 136.66 & 235.28 & 3.12 & 98.62 & 388 & 0.35 \\
\hline 0 & 0.61 & 0.59 & 0.59 & 23.93 & 23.93 & 19.78 & 152.50 & 152.50 & 0.00 & 0.00 & 388 & 0.39 \\
\hline 0 & 0.59 & 0.60 & 0.24 & 29.21 & 28.20 & 25.62 & 162.94 & 297.23 & 4.66 & 134.29 & 388 & 0.42 \\
\hline 0 & 0.84 & 0.82 & 0.34 & 30.56 & 30.97 & 27.78 & 98.73 & 281.15 & 5.95 & 182.42 & 388 & 0.25 \\
\hline 0 & 0.51 & 0.54 & 0.54 & 30.24 & 30.24 & 26.95 & 172.07 & 172.07 & 0.00 & 0.00 & 388 & 0.44 \\
\hline 0 & 0.18 & 0.13 & 0.13 & 28.11 & 28.11 & 27.00 & 237.13 & 237.13 & 0.00 & 0.00 & 388 & 0.61 \\
\hline 0 & -0.07 & 0.01 & 0.01 & 29.20 & 29.20 & 29.12 & 262.08 & 262.08 & 0.00 & 0.00 & 388 & 0.68 \\
\hline
\end{tabular}




\begin{tabular}{|c|c|c|c|c|c|c|c|c|c|c|c|c|}
\hline 0 & 0.32 & 0.35 & 0.53 & 20.76 & 24.83 & 19.11 & 92.31 & 233.36 & 6.19 & 141.05 & 388 & 0.24 \\
\hline 0 & 0.55 & 0.49 & 0.14 & 30.59 & 29.81 & 28.62 & 120.42 & 257.65 & 4.54 & 137.23 & 388 & 0.31 \\
\hline 0 & 0.22 & 0.25 & 0.25 & 24.36 & 24.36 & 21.03 & 299.09 & 299.09 & 0.00 & 0.00 & 388 & 0.77 \\
\hline 0 & 0.55 & 0.53 & 0.53 & 29.57 & 29.57 & 24.20 & 273.72 & 273.72 & 0.00 & 0.00 & 388 & 0.71 \\
\hline 0 & 0.54 & 0.50 & 0.22 & 28.84 & 28.69 & 26.39 & 133.42 & 289.88 & 5.44 & 156.46 & 388 & 0.34 \\
\hline 0 & 0.48 & 0.37 & 0.10 & 33.15 & 32.42 & 31.64 & 133.56 & 244.49 & 3.37 & 110.93 & 388 & 0.34 \\
\hline 0 & 0.91 & 0.85 & 0.72 & 28.73 & 30.35 & 24.95 & 119.27 & 207.68 & 3.03 & 88.41 & 388 & 0.31 \\
\hline 0 & 0.23 & 0.33 & 0.33 & 25.31 & 25.31 & 21.59 & 260.13 & 260.13 & 0.00 & 0.00 & 388 & 0.67 \\
\hline 0 & 0.90 & 0.83 & 0.46 & 32.38 & 32.42 & 28.06 & 156.50 & 288.43 & 4.09 & 131.92 & 388 & 0.40 \\
\hline 0 & 0.24 & 0.27 & 0.27 & 26.72 & 26.72 & 24.31 & 224.21 & 224.21 & 0.00 & 0.00 & 388 & 0.58 \\
\hline 0 & 0.49 & 0.45 & 0.45 & 28.19 & 28.19 & 23.08 & 292.84 & 292.84 & 0.00 & 0.00 & 388 & 0.75 \\
\hline 0 & 0.20 & 0.18 & 0.18 & 31.70 & 31.70 & 29.98 & 295.99 & 295.99 & 0.00 & 0.00 & 388 & 0.76 \\
\hline 0 & 0.43 & 0.44 & 0.30 & 25.99 & 26.27 & 22.78 & 178.26 & 281.44 & 3.99 & 103.19 & 388 & 0.46 \\
\hline 0 & 0.43 & 0.38 & 0.31 & 28.88 & 29.16 & 26.00 & 208.88 & 280.59 & 2.48 & 71.71 & 388 & 0.54 \\
\hline 0 & 0.50 & 0.41 & 0.31 & 29.79 & 30.89 & 28.37 & 100.11 & 243.98 & 4.74 & 143.87 & 388 & 0.26 \\
\hline 0 & 0.42 & 0.39 & 0.25 & 26.51 & 27.63 & 24.97 & 100.15 & 285.68 & 6.91 & 185.53 & 388 & 0.26 \\
\hline 0 & 0.84 & 0.81 & 0.49 & 33.29 & 34.60 & 30.58 & 105.76 & 267.88 & 4.79 & 162.13 & 388 & 0.27 \\
\hline 0 & 0.42 & 0.40 & 0.39 & 31.10 & 31.86 & 29.09 & 151.43 & 213.93 & 2.02 & 62.50 & 388 & 0.39 \\
\hline 0 & 0.38 & 0.49 & 0.49 & 28.92 & 28.92 & 24.42 & 240.77 & 240.77 & 0.00 & 0.00 & 388 & 0.62 \\
\hline 0 & 0.15 & 0.22 & 0.22 & 29.46 & 29.46 & 27.37 & 270.98 & 270.98 & 0.00 & 0.00 & 388 & 0.70 \\
\hline 0 & 0.24 & 0.24 & 0.16 & 27.25 & 26.88 & 25.10 & 236.06 & 290.82 & 2.02 & 54.76 & 388 & 0.61 \\
\hline 0 & 0.11 & 0.15 & 0.15 & 29.91 & 29.91 & 28.27 & 310.77 & 310.77 & 0.00 & 0.00 & 388 & 0.80 \\
\hline 0 & 0.54 & 0.48 & 0.25 & 28.23 & 28.61 & 25.98 & 126.35 & 291.68 & 5.80 & 165.33 & 388 & 0.33 \\
\hline 0 & 0.19 & 0.17 & 0.17 & 33.82 & 33.82 & 32.42 & 268.49 & 268.49 & 0.00 & 0.00 & 388 & 0.69 \\
\hline 0 & 0.07 & 0.11 & 0.12 & 24.52 & 24.97 & 23.42 & 241.26 & 300.85 & 2.42 & 59.59 & 388 & 0.62 \\
\hline 0 & 0.63 & 0.62 & 0.36 & 28.93 & 30.04 & 26.31 & 116.67 & 297.09 & 6.15 & 180.42 & 388 & 0.30 \\
\hline 0 & 0.36 & 0.42 & 0.42 & 28.38 & 28.38 & 23.95 & 277.03 & 277.03 & 0.00 & 0.00 & 388 & 0.71 \\
\hline 0 & 0.06 & 0.02 & 0.01 & 24.35 & 24.29 & 24.20 & 191.84 & 194.33 & 0.11 & 2.48 & 388 & 0.49 \\
\hline 0 & 0.27 & 0.49 & 0.49 & 26.63 & 26.63 & 20.40 & 292.12 & 292.12 & 0.00 & 0.00 & 388 & 0.75 \\
\hline 0 & 0.18 & 0.20 & 0.53 & 24.03 & 28.56 & 23.29 & 88.56 & 256.60 & 6.40 & 168.04 & 388 & 0.23 \\
\hline 0 & 0.23 & 0.17 & 0.10 & 24.79 & 25.11 & 24.09 & 98.30 & 251.90 & 6.25 & 153.60 & 388 & 0.25 \\
\hline 0 & 0.19 & 0.17 & 0.13 & 28.37 & 28.87 & 27.64 & 119.48 & 269.87 & 5.29 & 150.39 & 388 & 0.31 \\
\hline 0 & 0.25 & 0.24 & 0.24 & 32.81 & 32.81 & 30.64 & 288.16 & 288.16 & 0.00 & 0.00 & 388 & 0.74 \\
\hline 0 & 1.08 & 0.96 & 0.70 & 30.15 & 31.60 & 25.20 & 141.34 & 262.16 & 3.93 & 120.82 & 388 & 0.36 \\
\hline 0 & 0.00 & 0.07 & 0.07 & 27.73 & 27.73 & 26.95 & 297.80 & 297.80 & 0.00 & 0.00 & 388 & 0.77 \\
\hline 0 & 0.18 & 0.15 & -0.02 & 31.90 & 31.21 & 31.39 & 103.85 & 271.84 & 5.35 & 167.98 & 388 & 0.27 \\
\hline 0 & 0.39 & 0.27 & 0.27 & 28.48 & 28.48 & 25.89 & 261.93 & 261.93 & 0.00 & 0.00 & 388 & 0.68 \\
\hline 0 & 0.30 & 0.25 & 0.25 & 29.82 & 29.82 & 27.17 & 305.89 & 305.89 & 0.00 & 0.00 & 388 & 0.79 \\
\hline 0 & 0.38 & 0.43 & 0.43 & 28.96 & 28.96 & 24.45 & 274.93 & 274.93 & 0.00 & 0.00 & 388 & 0.71 \\
\hline 0 & 0.62 & 0.57 & 0.57 & 27.88 & 27.88 & 23.12 & 210.57 & 210.57 & 0.00 & 0.00 & 388 & 0.54 \\
\hline
\end{tabular}




\begin{tabular}{|c|c|c|c|c|c|c|c|c|c|c|c|c|}
\hline 0 & 0.72 & 0.62 & 0.44 & 30.15 & 30.68 & 26.59 & 162.77 & 264.95 & 3.38 & 102.18 & 388 & 0.42 \\
\hline 0 & 0.46 & 0.38 & 0.28 & 25.96 & 26.37 & 23.42 & 163.58 & 265.38 & 3.94 & 101.80 & 388 & 0.42 \\
\hline 0 & 0.42 & 0.32 & 0.29 & 27.26 & 28.09 & 25.37 & 156.98 & 249.61 & 3.38 & 92.64 & 388 & 0.40 \\
\hline 0 & 0.68 & 0.70 & 0.52 & 30.62 & 32.82 & 28.33 & 95.61 & 260.00 & 5.49 & 164.39 & 388 & 0.25 \\
\hline 0 & 0.56 & 0.62 & 0.62 & 32.24 & 32.24 & 26.75 & 261.35 & 261.35 & 0.00 & 0.00 & 388 & 0.67 \\
\hline 0 & 0.40 & 0.35 & 0.28 & 29.04 & 29.48 & 26.82 & 178.12 & 266.17 & 3.03 & 88.06 & 388 & 0.46 \\
\hline 0 & 0.82 & 0.96 & 0.99 & 30.76 & 32.43 & 27.39 & 100.70 & 149.66 & 1.56 & 48.96 & 388 & 0.26 \\
\hline 0 & 0.44 & 0.30 & 0.30 & 29.52 & 29.52 & 27.27 & 212.64 & 212.64 & 0.00 & 0.00 & 388 & 0.55 \\
\hline 0 & 0.06 & 0.19 & 0.19 & 28.53 & 28.53 & 26.78 & 254.75 & 254.75 & 0.00 & 0.00 & 388 & 0.66 \\
\hline 0 & 0.41 & 0.34 & 0.34 & 27.19 & 27.19 & 24.20 & 231.14 & 231.14 & 0.00 & 0.00 & 388 & 0.60 \\
\hline 0 & 0.75 & 0.67 & 0.40 & 29.31 & 30.85 & 27.10 & 90.78 & 268.66 & 5.93 & 177.88 & 388 & 0.23 \\
\hline 0 & 0.23 & 0.17 & 0.17 & 28.13 & 28.13 & 26.34 & 286.59 & 286.59 & 0.00 & 0.00 & 388 & 0.74 \\
\hline 0 & 0.26 & 0.35 & 0.35 & 27.16 & 27.27 & 23.23 & 285.81 & 289.91 & 0.16 & 4.10 & 388 & 0.74 \\
\hline 0 & 0.30 & 0.21 & 0.18 & 28.35 & 28.05 & 26.18 & 281.64 & 290.48 & 0.31 & 8.84 & 388 & 0.73 \\
\hline 0 & 0.46 & 0.28 & 0.28 & 30.60 & 30.60 & 28.09 & 265.65 & 265.65 & 0.00 & 0.00 & 388 & 0.68 \\
\hline 0 & 0.39 & 0.30 & 0.25 & 29.63 & 29.91 & 27.55 & 196.93 & 273.35 & 2.58 & 76.42 & 388 & 0.51 \\
\hline 0 & 0.62 & 0.54 & 0.40 & 29.58 & 29.21 & 25.34 & 214.61 & 266.88 & 1.77 & 52.27 & 388 & 0.55 \\
\hline 0 & 0.52 & 0.53 & 0.53 & 30.25 & 30.25 & 24.70 & 287.59 & 287.59 & 0.00 & 0.00 & 388 & 0.74 \\
\hline 0 & 0.21 & 0.18 & 0.05 & 28.40 & 27.86 & 27.31 & 173.69 & 287.12 & 4.04 & 113.43 & 388 & 0.45 \\
\hline 0 & 0.24 & 0.27 & 0.27 & 30.95 & 30.95 & 28.31 & 294.46 & 294.46 & 0.00 & 0.00 & 388 & 0.76 \\
\hline 0 & 0.59 & 0.56 & 0.47 & 27.84 & 29.96 & 25.42 & 115.17 & 265.28 & 5.25 & 150.11 & 388 & 0.30 \\
\hline 0 & 0.65 & 0.54 & 0.37 & 29.27 & 29.29 & 25.17 & 205.45 & 302.65 & 3.33 & 97.20 & 388 & 0.53 \\
\hline 0 & 0.43 & 0.39 & 0.39 & 26.91 & 26.91 & 24.03 & 189.90 & 189.90 & 0.00 & 0.00 & 388 & 0.49 \\
\hline 0 & 0.45 & 0.36 & 0.14 & 29.85 & 29.46 & 28.17 & 136.05 & 264.30 & 4.34 & 128.25 & 388 & 0.35 \\
\hline 0 & 0.48 & 0.44 & 0.40 & 25.91 & 27.14 & 22.31 & 194.86 & 300.93 & 4.03 & 106.08 & 388 & 0.50 \\
\hline 0 & 0.78 & 0.74 & 0.54 & 29.84 & 30.43 & 27.37 & 94.70 & 163.04 & 2.27 & 68.34 & 388 & 0.24 \\
\hline 0 & 0.92 & 0.83 & 0.63 & 29.46 & 30.55 & 25.67 & 124.12 & 217.99 & 3.18 & 93.87 & 388 & 0.32 \\
\hline 0 & 0.64 & 0.53 & 0.53 & 31.74 & 31.74 & 26.23 & 302.58 & 302.58 & 0.00 & 0.00 & 388 & 0.78 \\
\hline 0 & 0.39 & 0.21 & 0.16 & 31.60 & 31.49 & 30.45 & 171.44 & 198.35 & 0.86 & 26.91 & 388 & 0.44 \\
\hline 1 & 0.04 & 0.18 & 0.33 & 23.76 & 26.39 & 22.53 & 160.09 & 281.42 & 4.89 & 121.33 & 388 & 0.41 \\
\hline 1 & 0.42 & 0.38 & 0.37 & 27.54 & 27.85 & 24.78 & 187.77 & 218.26 & 1.11 & 30.50 & 388 & 0.48 \\
\hline 1 & 0.36 & 0.22 & 0.22 & 25.68 & 25.68 & 23.64 & 233.41 & 233.41 & 0.00 & 0.00 & 388 & 0.60 \\
\hline 1 & 0.33 & 0.27 & 0.19 & 27.12 & 27.49 & 25.42 & 164.66 & 293.60 & 4.74 & 128.95 & 388 & 0.42 \\
\hline 1 & 0.42 & 0.32 & 0.32 & 29.80 & 29.80 & 26.28 & 304.49 & 304.49 & 0.00 & 0.00 & 388 & 0.78 \\
\hline 1 & 0.12 & 0.20 & 0.22 & 26.50 & 26.75 & 24.42 & 264.70 & 271.31 & 0.26 & 6.61 & 388 & 0.68 \\
\hline 1 & 0.40 & 0.35 & 0.26 & 27.57 & 27.56 & 24.73 & 212.60 & 282.02 & 2.53 & 69.42 & 388 & 0.55 \\
\hline 1 & 0.58 & 0.53 & 0.33 & 28.76 & 28.91 & 26.06 & 138.27 & 235.62 & 3.38 & 97.36 & 388 & 0.36 \\
\hline 1 & 0.59 & 0.50 & 0.28 & 27.26 & 27.55 & 24.89 & 123.68 & 248.42 & 4.54 & 124.74 & 388 & 0.32 \\
\hline 1 & 0.38 & 0.24 & 0.24 & 25.39 & 25.39 & 22.28 & 312.69 & 312.69 & 0.00 & 0.00 & 388 & 0.81 \\
\hline $\begin{array}{c}\mathrm{Me} \\
\text { an }\end{array}$ & 0.42 & 0.39 & 0.33 & 28.66 & 28.96 & 25.98 & 200.46 & 257.02 & 1.97 & 56.56 & 388 & 0.52 \\
\hline
\end{tabular}




\begin{tabular}{|l|l|l|l|l|l|l|l|l|l|l|l|l|}
\hline Std & 0.23 & 0.20 & 0.17 & 2.39 & 2.23 & 2.58 & 68.86 & 40.21 & 2.29 & 65.42 & 0 & 0.18 \\
\hline
\end{tabular}


Table 7: Carp Road (E-NS).

\begin{tabular}{|c|c|c|c|c|c|c|c|c|c|c|c|c|}
\hline $\begin{array}{l}\text { Tru } \\
\text { cks }\end{array}$ & $\begin{array}{c}\text { Mean } \\
\text { Decelera } \\
\text { tion }\end{array}$ & \begin{tabular}{|c|} 
Adjusted \\
Overall \\
Decelerati \\
on \\
\end{tabular} & $\begin{array}{c}\text { Original } \\
\text { Overall } \\
\text { Deceleration }\end{array}$ & $\begin{array}{l}\text { Initial } \\
\text { Speed }\end{array}$ & $\begin{array}{c}\text { Diverg } \\
\text { e } \\
\text { Speed }\end{array}$ & $\begin{array}{c}\text { Final } \\
\text { Speed }\end{array}$ & $\begin{array}{l}\text { Decelera } \\
\text { tion } \\
\text { Distance }\end{array}$ & $\begin{array}{l}\text { Decelerati } \\
\text { on Length }\end{array}$ & $\begin{array}{l}\text { Decelerati } \\
\text { on Time }\end{array}$ & \begin{tabular}{|c} 
Distance \\
to \\
Decelera \\
te
\end{tabular} & $\begin{array}{c}\mathrm{SCL} \\
\text { Lengt } \\
\mathrm{h}\end{array}$ & $\begin{array}{l}\text { Utilizati } \\
\text { on Rate }\end{array}$ \\
\hline 0 & 0.19 & 0.13 & 0.13 & 31.58 & 31.58 & 30.15 & 344.26 & 344.26 & 0.00 & 0.00 & 430 & 0.80 \\
\hline 0 & 0.25 & 0.21 & 0.19 & 29.61 & 29.50 & 27.47 & 289.49 & 298.40 & 0.30 & 8.90 & 430 & 0.67 \\
\hline 0 & 0.59 & 0.49 & 0.22 & 31.00 & 31.58 & 29.01 & 120.42 & 352.41 & 7.36 & 231.98 & 430 & 0.28 \\
\hline 0 & 0.30 & 0.27 & 0.16 & 27.81 & 27.86 & 26.00 & 179.12 & 310.16 & 4.68 & 131.04 & 430 & 0.42 \\
\hline 0 & 0.51 & 0.39 & 0.39 & 30.25 & 30.25 & 26.17 & 295.80 & 295.80 & 0.00 & 0.00 & 430 & 0.69 \\
\hline 0 & 0.30 & 0.37 & 0.06 & 29.28 & 28.89 & 28.24 & 80.06 & 302.30 & 7.68 & 222.24 & 430 & 0.19 \\
\hline 0 & 0.79 & 0.32 & 0.15 & 32.92 & 32.03 & 30.12 & 277.43 & 392.83 & 3.50 & 115.40 & 430 & 0.65 \\
\hline 0 & 0.64 & 0.55 & 0.37 & 33.39 & 33.50 & 28.95 & 252.67 & 382.52 & 3.90 & 129.85 & 430 & 0.59 \\
\hline 0 & 0.53 & 0.35 & 0.32 & 29.14 & 29.81 & 26.44 & 213.95 & 297.03 & 2.83 & 83.07 & 430 & 0.50 \\
\hline 0 & 0.45 & 0.48 & 0.29 & 26.89 & 28.97 & 24.82 & 113.10 & 380.27 & 9.72 & 267.17 & 430 & 0.26 \\
\hline 0 & 0.43 & 0.30 & 0.27 & 25.72 & 26.42 & 24.25 & 121.05 & 204.17 & 3.23 & 83.12 & 430 & 0.28 \\
\hline 0 & 0.69 & 0.63 & 0.24 & 30.47 & 30.22 & 27.14 & 151.81 & 381.94 & 7.50 & 230.13 & 430 & 0.35 \\
\hline 0 & 0.77 & 0.61 & 0.35 & 32.36 & 31.39 & 27.86 & 220.67 & 303.33 & 2.52 & 82.65 & 430 & 0.51 \\
\hline 0 & 0.75 & 0.75 & 0.28 & 30.03 & 31.22 & 27.75 & 86.80 & 368.02 & 9.11 & 281.22 & 430 & 0.20 \\
\hline 0 & 0.63 & 0.57 & 0.33 & 28.75 & 29.92 & 26.23 & 122.36 & 315.85 & 6.50 & 193.48 & 430 & 0.28 \\
\hline 0 & 0.67 & 0.66 & 0.33 & 31.11 & 32.22 & 28.40 & 122.14 & 353.13 & 7.30 & 230.98 & 430 & 0.28 \\
\hline 0 & 0.37 & 0.35 & 0.15 & 29.47 & 29.97 & 28.11 & 113.49 & 370.43 & 8.53 & 256.94 & 430 & 0.26 \\
\hline 0 & 0.37 & 0.28 & 0.16 & 31.00 & 30.78 & 28.83 & 235.42 & 358.30 & 3.90 & 122.88 & 430 & 0.55 \\
\hline 0 & -0.03 & 0.21 & 0.21 & 29.25 & 29.25 & 26.40 & 369.20 & 369.20 & 0.00 & 0.00 & 430 & 0.86 \\
\hline 0 & 0.42 & 0.37 & 0.18 & 27.28 & 27.75 & 25.63 & 116.80 & 324.62 & 7.57 & 207.81 & 430 & 0.27 \\
\hline 0 & 0.67 & 0.57 & 0.34 & 32.22 & 31.89 & 28.01 & 222.72 & 348.64 & 3.96 & 125.92 & 430 & 0.52 \\
\hline 0 & 0.54 & 0.26 & 0.26 & 31.31 & 31.31 & 28.61 & 313.68 & 313.68 & 0.00 & 0.00 & 430 & 0.73 \\
\hline 0 & 0.30 & 0.21 & 0.21 & 27.75 & 27.75 & 25.05 & 348.14 & 348.14 & 0.00 & 0.00 & 430 & 0.81 \\
\hline 0 & 0.84 & 0.77 & 0.39 & 29.09 & 30.14 & 25.98 & 110.29 & 299.45 & 6.37 & 189.15 & 430 & 0.26 \\
\hline 0 & 0.43 & 0.36 & 0.36 & 31.22 & 31.22 & 26.97 & 344.10 & 344.10 & 0.00 & 0.00 & 430 & 0.80 \\
\hline 0 & 0.34 & 0.35 & 0.19 & 27.72 & 28.33 & 26.04 & 130.23 & 326.71 & 7.09 & 196.47 & 430 & 0.30 \\
\hline 0 & 0.42 & 0.48 & 0.10 & 28.20 & 27.95 & 26.79 & 79.95 & 320.93 & 8.56 & 240.98 & 430 & 0.19 \\
\hline 0 & 0.39 & 0.35 & 0.35 & 33.39 & 33.39 & 29.39 & 357.97 & 357.97 & 0.00 & 0.00 & 430 & 0.83 \\
\hline 0 & 0.23 & 0.16 & 0.05 & 32.75 & 32.22 & 31.70 & 213.01 & 336.29 & 3.81 & 123.28 & 430 & 0.50 \\
\hline 0 & 0.86 & 0.76 & 0.29 & 29.92 & 30.03 & 26.50 & 126.89 & 348.85 & 7.37 & 221.95 & 430 & 0.30 \\
\hline 0 & 0.33 & 0.26 & 0.26 & 27.47 & 27.47 & 24.11 & 333.48 & 333.48 & 0.00 & 0.00 & 430 & 0.78 \\
\hline 0 & 0.18 & 0.15 & 0.15 & 31.03 & 31.03 & 29.29 & 343.24 & 343.24 & 0.00 & 0.00 & 430 & 0.80 \\
\hline 0 & 0.49 & 0.32 & 0.14 & 33.09 & 33.47 & 32.02 & 107.18 & 354.44 & 7.42 & 247.26 & 430 & 0.25 \\
\hline 0 & 0.64 & 0.32 & 0.32 & 28.97 & 28.97 & 24.63 & 367.45 & 367.45 & 0.00 & 0.00 & 430 & 0.85 \\
\hline 0 & 0.56 & 0.34 & 0.21 & 30.78 & 30.14 & 28.11 & 232.14 & 285.53 & 1.75 & 53.39 & 430 & 0.54 \\
\hline
\end{tabular}




\begin{tabular}{|c|c|c|c|c|c|c|c|c|c|c|c|c|}
\hline 0 & 0.67 & 0.51 & 0.33 & 31.11 & 32.58 & 28.64 & 145.68 & 369.18 & 7.05 & 223.50 & 430 & 0.34 \\
\hline 0 & 0.61 & 0.24 & 0.24 & 31.20 & 31.20 & 28.87 & 301.12 & 301.12 & 0.00 & 0.00 & 430 & 0.70 \\
\hline 0 & 0.39 & 0.11 & 0.11 & 28.47 & 28.47 & 27.11 & 363.72 & 363.72 & 0.00 & 0.00 & 430 & 0.85 \\
\hline 0 & 0.28 & 0.23 & 0.14 & 29.56 & 29.61 & 28.00 & 198.99 & 332.97 & 4.55 & 133.98 & 430 & 0.46 \\
\hline 0 & 0.46 & 0.39 & 0.39 & 34.97 & 34.97 & 32.63 & 203.88 & 203.88 & 0.00 & 0.00 & 430 & 0.47 \\
\hline 0 & 0.15 & 0.09 & 0.09 & 29.25 & 29.25 & 28.00 & 390.00 & 390.00 & 0.00 & 0.00 & 430 & 0.91 \\
\hline 0 & 0.36 & 0.45 & 0.45 & 32.25 & 32.25 & 26.89 & 350.16 & 350.16 & 0.00 & 0.00 & 430 & 0.81 \\
\hline 0 & 0.36 & 0.16 & 0.16 & 26.86 & 26.86 & 25.08 & 297.26 & 297.26 & 0.00 & 0.00 & 430 & 0.69 \\
\hline 0 & 0.54 & 0.52 & 0.32 & 28.53 & 29.97 & 25.77 & 143.11 & 366.33 & 7.59 & 223.22 & 430 & 0.33 \\
\hline 0 & 0.24 & 0.24 & 0.24 & 30.08 & 30.08 & 26.70 & 397.68 & 397.68 & 0.00 & 0.00 & 430 & 0.92 \\
\hline 0 & 0.05 & 0.29 & 0.31 & 34.34 & 34.56 & 31.90 & 271.32 & 278.16 & 0.21 & 6.84 & 430 & 0.63 \\
\hline 0 & 0.43 & 0.32 & 0.28 & 28.92 & 29.22 & 25.25 & 312.31 & 383.16 & 2.44 & 70.85 & 430 & 0.73 \\
\hline 0 & 0.28 & 0.21 & 0.21 & 26.45 & 26.45 & 23.78 & 319.92 & 319.92 & 0.00 & 0.00 & 430 & 0.74 \\
\hline 0 & 0.92 & 0.43 & 0.43 & 33.72 & 33.72 & 29.48 & 315.10 & 315.10 & 0.00 & 0.00 & 430 & 0.73 \\
\hline 0 & 0.71 & 0.70 & 0.21 & 29.61 & 30.61 & 27.70 & 78.02 & 401.09 & 10.64 & 323.07 & 430 & 0.18 \\
\hline 0 & 0.22 & 0.33 & 0.33 & 28.97 & 28.97 & 25.43 & 297.65 & 297.65 & 0.00 & 0.00 & 430 & 0.69 \\
\hline 0 & 0.46 & 0.43 & 0.43 & 30.83 & 30.83 & 26.09 & 315.97 & 315.97 & 0.00 & 0.00 & 430 & 0.73 \\
\hline 0 & 0.33 & 0.24 & 0.23 & 26.31 & 27.53 & 24.86 & 152.31 & 309.99 & 5.90 & 157.68 & 430 & 0.35 \\
\hline 0 & 0.18 & 0.09 & 0.09 & 25.81 & 25.81 & 24.57 & 341.98 & 341.98 & 0.00 & 0.00 & 430 & 0.80 \\
\hline 0 & 0.22 & 0.18 & 0.14 & 26.97 & 26.58 & 24.89 & 298.94 & 305.85 & 0.25 & 6.91 & 430 & 0.70 \\
\hline 0 & 0.45 & 0.31 & 0.31 & 30.31 & 30.31 & 26.83 & 322.87 & 322.87 & 0.00 & 0.00 & 430 & 0.75 \\
\hline 0 & 0.42 & 0.34 & 0.34 & 29.14 & 29.14 & 26.50 & 217.88 & 217.88 & 0.00 & 0.00 & 430 & 0.51 \\
\hline 0 & 1.02 & 0.95 & 0.81 & 32.36 & 33.50 & 24.45 & 234.26 & 325.79 & 2.72 & 91.52 & 430 & 0.54 \\
\hline 0 & 0.29 & 0.23 & -0.01 & 31.28 & 30.28 & 30.43 & 112.05 & 347.50 & 7.62 & 235.44 & 430 & 0.26 \\
\hline 0 & 0.44 & 0.30 & 0.15 & 23.56 & 24.03 & 22.12 & 109.14 & 293.85 & 7.69 & 184.71 & 430 & 0.25 \\
\hline 0 & 0.22 & 0.19 & 0.19 & 29.06 & 29.06 & 26.67 & 357.75 & 357.75 & 0.00 & 0.00 & 430 & 0.83 \\
\hline 0 & 0.38 & 0.22 & 0.19 & 31.75 & 31.89 & 29.78 & 281.36 & 344.06 & 1.98 & 62.69 & 430 & 0.65 \\
\hline 0 & 0.23 & 0.22 & 0.18 & 29.78 & 29.56 & 27.61 & 287.08 & 304.15 & 0.51 & 17.06 & 430 & 0.67 \\
\hline 0 & 0.48 & 0.43 & 0.27 & 30.86 & 32.33 & 28.99 & 131.89 & 387.56 & 8.10 & 255.66 & 430 & 0.31 \\
\hline 0 & 0.64 & 0.43 & 0.23 & 27.78 & 28.08 & 25.11 & 165.54 & 342.72 & 6.29 & 177.18 & 430 & 0.38 \\
\hline 0 & 0.22 & 0.15 & 0.06 & 27.89 & 27.58 & 26.83 & 199.23 & 363.40 & 5.97 & 164.17 & 430 & 0.46 \\
\hline 0 & 0.49 & 0.44 & 0.29 & 28.64 & 29.64 & 26.51 & 132.18 & 305.22 & 5.90 & 173.03 & 430 & 0.31 \\
\hline 0 & 0.69 & 0.73 & 0.34 & 30.31 & 31.42 & 27.17 & 123.21 & 371.03 & 8.08 & 247.81 & 430 & 0.29 \\
\hline 0 & 0.52 & 0.44 & 0.18 & 30.31 & 30.97 & 28.74 & 105.97 & 378.21 & 8.81 & 272.24 & 430 & 0.25 \\
\hline 0 & 0.44 & 0.40 & 0.36 & 27.06 & 29.36 & 24.86 & 141.17 & 334.59 & 6.92 & 193.42 & 430 & 0.33 \\
\hline 0 & 0.48 & 0.46 & 0.25 & 30.72 & 31.31 & 28.70 & 130.82 & 309.76 & 5.74 & 178.93 & 430 & 0.30 \\
\hline 0 & 0.94 & 0.85 & 0.53 & 33.28 & 34.31 & 28.55 & 170.86 & 343.06 & 5.08 & 172.20 & 430 & 0.40 \\
\hline 0 & 0.65 & 0.50 & 0.29 & 29.45 & 29.92 & 26.29 & 174.27 & 357.06 & 6.16 & 182.79 & 430 & 0.41 \\
\hline 0 & 0.50 & 0.47 & 0.26 & 26.78 & 27.50 & 24.54 & 120.46 & 300.85 & 6.61 & 180.38 & 430 & 0.28 \\
\hline 0 & 0.36 & 0.30 & 0.25 & 27.83 & 27.86 & 24.70 & 277.78 & 326.83 & 1.77 & 49.04 & 430 & 0.65 \\
\hline
\end{tabular}




\begin{tabular}{|c|c|c|c|c|c|c|c|c|c|c|c|c|}
\hline 0 & 0.45 & 0.39 & 0.21 & 27.06 & 28.28 & 25.56 & 101.87 & 356.17 & 9.18 & 254.30 & 430 & 0.24 \\
\hline 0 & 0.63 & 0.60 & 0.38 & 31.03 & 32.14 & 28.49 & 126.37 & 289.63 & 5.20 & 163.26 & 430 & 0.29 \\
\hline 0 & 0.66 & 0.53 & 0.32 & 28.95 & 30.11 & 26.61 & 123.49 & 316.11 & 6.51 & 192.61 & 430 & 0.29 \\
\hline 0 & 0.63 & 0.53 & 0.29 & 29.89 & 31.08 & 27.74 & 117.32 & 336.61 & 7.17 & 219.28 & 430 & 0.27 \\
\hline 0 & 0.34 & 0.15 & 0.15 & 30.56 & 30.56 & 29.00 & 318.05 & 318.05 & 0.00 & 0.00 & 430 & 0.74 \\
\hline 0 & 0.29 & 0.23 & 0.18 & 28.00 & 28.58 & 26.55 & 175.38 & 312.28 & 4.84 & 136.90 & 430 & 0.41 \\
\hline 0 & 0.07 & 0.08 & 0.30 & 23.53 & 27.39 & 23.16 & 106.51 & 356.66 & 9.90 & 250.15 & 430 & 0.25 \\
\hline 0 & 0.39 & 0.31 & 0.21 & 31.75 & 31.58 & 29.33 & 235.78 & 326.99 & 2.88 & 91.20 & 430 & 0.55 \\
\hline 0 & 0.19 & 0.14 & 0.04 & 27.45 & 26.83 & 26.39 & 205.38 & 312.31 & 3.94 & 106.93 & 430 & 0.48 \\
\hline 1 & 0.24 & 0.22 & 0.06 & 28.09 & 28.00 & 27.28 & 102.30 & 356.48 & 9.01 & 254.18 & 430 & 0.24 \\
\hline 1 & 0.18 & 0.12 & 0.04 & 27.34 & 27.42 & 26.88 & 103.58 & 349.54 & 8.98 & 245.96 & 430 & 0.24 \\
\hline 1 & 0.15 & 0.05 & 0.05 & 27.86 & 27.86 & 27.38 & 288.98 & 288.98 & 0.00 & 0.00 & 430 & 0.67 \\
\hline 1 & 0.41 & 0.33 & 0.10 & 28.72 & 28.64 & 27.42 & 111.46 & 330.38 & 7.67 & 218.92 & 430 & 0.26 \\
\hline 1 & 0.58 & 0.40 & 0.32 & 25.78 & 27.20 & 23.03 & 167.23 & 331.87 & 6.28 & 164.64 & 430 & 0.39 \\
\hline 1 & 0.68 & 0.45 & 0.26 & 26.86 & 27.00 & 23.88 & 170.31 & 312.20 & 5.26 & 141.90 & 430 & 0.40 \\
\hline 1 & 0.08 & 0.07 & 0.11 & 25.78 & 26.42 & 24.76 & 345.39 & 390.66 & 1.70 & 45.26 & 430 & 0.80 \\
\hline 1 & -0.01 & 0.03 & 0.03 & 26.14 & 26.14 & 25.86 & 271.34 & 271.34 & 0.00 & 0.00 & 430 & 0.63 \\
\hline 1 & 0.43 & 0.03 & 0.03 & 29.31 & 29.31 & 28.98 & 341.18 & 341.18 & 0.00 & 0.00 & 430 & 0.79 \\
\hline 1 & 0.32 & 0.17 & 0.17 & 22.61 & 22.61 & 20.08 & 327.13 & 327.13 & 0.00 & 0.00 & 430 & 0.76 \\
\hline 1 & 0.79 & 0.70 & 0.31 & 26.28 & 26.86 & 22.33 & 136.16 & 374.51 & 8.86 & 238.35 & 430 & 0.32 \\
\hline 1 & 0.34 & 0.25 & 0.25 & 26.61 & 26.61 & 23.78 & 272.23 & 272.23 & 0.00 & 0.00 & 430 & 0.63 \\
\hline 1 & 0.61 & 0.52 & 0.21 & 26.09 & 26.17 & 23.45 & 125.54 & 326.87 & 7.74 & 201.33 & 430 & 0.29 \\
\hline 1 & 0.46 & 0.33 & 0.18 & 26.83 & 26.58 & 24.44 & 186.78 & 307.57 & 4.53 & 120.80 & 430 & 0.43 \\
\hline $\begin{array}{c}\mathrm{Me} \\
\text { an }\end{array}$ & 0.44 & 0.36 & 0.24 & 29.26 & 29.59 & 26.84 & 216.40 & 331.78 & 3.95 & 115.38 & 430 & 0.50 \\
\hline Std & 0.22 & 0.19 & 0.12 & 2.42 & 2.34 & 2.25 & 94.62 & 37.74 & 3.46 & 100.74 & 0 & 0.22 \\
\hline
\end{tabular}




\section{Table 8: Terry Fox Drive (W-NS).}

\begin{tabular}{|c|c|c|c|c|c|c|c|c|c|c|c|c|}
\hline $\begin{array}{l}\text { Tru } \\
\text { cks }\end{array}$ & $\begin{array}{l}\text { Mean } \\
\text { Decelera } \\
\text { tion }\end{array}$ & $\begin{array}{c}\text { Adjusted } \\
\text { Overall } \\
\text { Deceleration }\end{array}$ & \begin{tabular}{|c|} 
Original \\
Overall \\
Decelerati \\
on \\
\end{tabular} & $\begin{array}{l}\text { Initial } \\
\text { Speed }\end{array}$ & $\begin{array}{c}\text { Diverg } \\
\text { e } \\
\text { Speed }\end{array}$ & $\begin{array}{c}\text { Final } \\
\text { Speed }\end{array}$ & $\begin{array}{l}\text { Decelera } \\
\text { tion } \\
\text { Distance }\end{array}$ & $\begin{array}{l}\text { Decelerati } \\
\text { on Length }\end{array}$ & $\begin{array}{l}\text { Decelerati } \\
\text { on Time }\end{array}$ & $\begin{array}{c}\text { Distance } \\
\text { to } \\
\text { Decelera } \\
\text { te }\end{array}$ & $\begin{array}{c}\mathrm{SCL} \\
\text { Lengt } \\
\mathrm{h}\end{array}$ & $\begin{array}{l}\text { Utilizati } \\
\text { on Rate }\end{array}$ \\
\hline 0 & 0.39 & 0.37 & 0.18 & 31.00 & 31.64 & 29.67 & 108.32 & 346.20 & 7.53 & 237.87 & 446 & 0.24 \\
\hline 0 & 0.43 & 0.36 & 0.36 & 30.92 & 30.92 & 28.86 & 169.59 & 171.16 & 0.05 & 1.57 & 446 & 0.38 \\
\hline 0 & 0.32 & 0.27 & -0.01 & 29.81 & 28.81 & 28.86 & 101.51 & 324.89 & 7.56 & 223.38 & 446 & 0.23 \\
\hline 0 & 0.74 & 0.70 & 0.43 & 28.89 & 29.42 & 25.59 & 126.86 & 245.40 & 4.08 & 118.54 & 446 & 0.28 \\
\hline 0 & 0.78 & 0.57 & 0.43 & 30.67 & 31.06 & 26.84 & 195.40 & 291.57 & 3.03 & 96.18 & 446 & 0.44 \\
\hline 0 & 0.70 & 0.62 & 0.24 & 26.84 & 27.03 & 23.89 & 119.71 & 337.50 & 8.06 & 217.78 & 446 & 0.27 \\
\hline 0 & 0.17 & 0.14 & 0.14 & 29.81 & 29.81 & 28.39 & 284.61 & 284.61 & 0.00 & 0.00 & 446 & 0.64 \\
\hline 0 & 0.26 & 0.20 & 0.19 & 31.92 & 31.92 & 30.09 & 291.36 & 292.97 & 0.05 & 1.61 & 446 & 0.65 \\
\hline 0 & 0.61 & 0.62 & 0.62 & 35.92 & 35.92 & 31.09 & 261.12 & 261.12 & 0.00 & 0.00 & 446 & 0.59 \\
\hline 0 & 0.50 & 0.20 & 0.20 & 31.58 & 31.58 & 29.42 & 329.85 & 329.85 & 0.00 & 0.00 & 446 & 0.74 \\
\hline 0 & 0.33 & 0.17 & 0.17 & 23.95 & 23.95 & 22.03 & 261.67 & 261.67 & 0.00 & 0.00 & 446 & 0.59 \\
\hline 0 & 0.35 & 0.26 & 0.26 & 26.47 & 26.47 & 22.92 & 340.40 & 340.40 & 0.00 & 0.00 & 446 & 0.76 \\
\hline 0 & 0.18 & 0.13 & 0.13 & 26.61 & 26.61 & 25.53 & 221.65 & 221.65 & 0.00 & 0.00 & 446 & 0.50 \\
\hline 0 & 0.83 & 0.74 & 0.36 & 32.48 & 33.75 & 29.81 & 112.62 & 349.20 & 7.13 & 236.58 & 446 & 0.25 \\
\hline 0 & 0.18 & 0.07 & 0.00 & 27.31 & 26.81 & 26.81 & 185.49 & 323.43 & 5.07 & 137.95 & 446 & 0.42 \\
\hline 0 & 0.27 & 0.24 & 0.24 & 30.67 & 30.67 & 28.70 & 247.66 & 247.66 & 0.00 & 0.00 & 446 & 0.56 \\
\hline 0 & 0.25 & 0.17 & 0.17 & 27.53 & 27.53 & 25.34 & 333.34 & 333.34 & 0.00 & 0.00 & 446 & 0.75 \\
\hline 0 & 0.29 & 0.25 & 0.25 & 30.81 & 30.81 & 28.03 & 326.12 & 326.12 & 0.00 & 0.00 & 446 & 0.73 \\
\hline 0 & 0.78 & 0.72 & 0.37 & 29.47 & 30.75 & 26.81 & 104.15 & 314.38 & 6.91 & 210.22 & 446 & 0.23 \\
\hline 0 & 0.15 & 0.10 & 0.04 & 27.97 & 27.45 & 27.06 & 251.20 & 275.54 & 0.86 & 24.34 & 446 & 0.56 \\
\hline 0 & 0.33 & 0.32 & 0.11 & 29.61 & 29.47 & 28.14 & 131.26 & 350.75 & 7.38 & 219.48 & 446 & 0.29 \\
\hline 0 & 0.76 & 0.62 & 0.38 & 35.34 & 35.09 & 31.81 & 190.57 & 288.96 & 2.78 & 98.40 & 446 & 0.43 \\
\hline 0 & 0.73 & 0.65 & 0.30 & 34.11 & 34.39 & 31.81 & 117.81 & 285.64 & 4.87 & 167.83 & 446 & 0.26 \\
\hline 0 & 0.52 & 0.54 & 0.26 & 28.61 & 29.28 & 26.42 & 110.26 & 310.31 & 6.86 & 200.05 & 446 & 0.25 \\
\hline 0 & 0.57 & 0.45 & 0.30 & 25.84 & 26.06 & 23.17 & 145.06 & 238.99 & 3.63 & 93.94 & 446 & 0.33 \\
\hline 0 & 0.57 & 0.64 & 0.64 & 28.70 & 28.70 & 25.34 & 141.35 & 141.35 & 0.00 & 0.00 & 446 & 0.32 \\
\hline 0 & 0.21 & 0.15 & 0.15 & 27.28 & 27.28 & 25.48 & 325.35 & 325.35 & 0.00 & 0.00 & 446 & 0.73 \\
\hline 0 & 0.12 & 0.18 & 0.20 & 28.00 & 28.45 & 27.39 & 95.14 & 149.08 & 1.91 & 53.94 & 446 & 0.21 \\
\hline 0 & 0.37 & 0.22 & 0.06 & 28.09 & 27.22 & 26.53 & 189.96 & 333.98 & 5.15 & 144.02 & 446 & 0.43 \\
\hline 0 & 0.57 & 0.54 & 0.38 & 25.20 & 25.64 & 20.53 & 197.98 & 314.68 & 4.58 & 116.71 & 446 & 0.44 \\
\hline 0 & 0.23 & 0.20 & 0.16 & 32.45 & 32.17 & 30.64 & 277.24 & 301.84 & 0.76 & 24.60 & 446 & 0.62 \\
\hline 0 & -0.06 & 0.13 & 0.13 & 30.09 & 30.09 & 29.20 & 207.71 & 207.71 & 0.00 & 0.00 & 446 & 0.47 \\
\hline 0 & 0.41 & 0.42 & 0.27 & 28.75 & 29.95 & 27.23 & 101.51 & 292.07 & 6.51 & 190.55 & 446 & 0.23 \\
\hline 0 & 0.41 & 0.11 & 0.11 & 29.17 & 29.17 & 28.89 & 72.60 & 72.60 & 0.00 & 0.00 & 446 & 0.16 \\
\hline 0 & 0.77 & 0.36 & 0.36 & 32.42 & 32.42 & 28.95 & 300.91 & 300.91 & 0.00 & 0.00 & 446 & 0.67 \\
\hline
\end{tabular}




\begin{tabular}{|c|c|c|c|c|c|c|c|c|c|c|c|c|}
\hline 0 & 0.21 & 0.19 & 0.19 & 29.86 & 29.86 & 27.81 & 316.14 & 316.14 & 0.00 & 0.00 & 446 & 0.71 \\
\hline 0 & 0.48 & 0.47 & 0.54 & 27.95 & 30.92 & 24.70 & 182.26 & 317.37 & 4.58 & 135.11 & 446 & 0.41 \\
\hline 0 & 0.43 & 0.43 & 0.32 & 27.47 & 29.64 & 25.42 & 127.32 & 362.22 & 8.40 & 234.89 & 446 & 0.29 \\
\hline 0 & 0.64 & 0.52 & 0.44 & 32.75 & 32.81 & 28.78 & 235.25 & 283.88 & 1.47 & 48.63 & 446 & 0.53 \\
\hline 0 & 0.47 & 0.36 & 0.36 & 29.11 & 29.11 & 24.61 & 334.44 & 334.44 & 0.00 & 0.00 & 446 & 0.75 \\
\hline 0 & 0.42 & 0.37 & 0.12 & 28.34 & 28.31 & 26.92 & 104.78 & 312.46 & 7.36 & 207.68 & 446 & 0.23 \\
\hline 0 & 0.05 & 0.12 & 0.12 & 31.58 & 31.58 & 30.37 & 304.33 & 304.33 & 0.00 & 0.00 & 446 & 0.68 \\
\hline 0 & 0.40 & 0.33 & 0.33 & 26.95 & 26.95 & 23.11 & 289.91 & 289.91 & 0.00 & 0.00 & 446 & 0.65 \\
\hline 0 & 0.05 & 0.01 & -0.10 & 27.64 & 26.47 & 27.56 & 160.85 & 307.69 & 5.41 & 146.85 & 446 & 0.36 \\
\hline 0 & 0.36 & 0.17 & 0.17 & 31.14 & 31.14 & 29.34 & 329.79 & 329.79 & 0.00 & 0.00 & 446 & 0.74 \\
\hline 0 & 0.33 & 0.20 & 0.17 & 28.42 & 28.47 & 26.34 & 281.91 & 345.40 & 2.27 & 63.48 & 446 & 0.63 \\
\hline 0 & 0.38 & 0.20 & 0.20 & 30.58 & 30.58 & 28.64 & 293.79 & 293.79 & 0.00 & 0.00 & 446 & 0.66 \\
\hline 0 & 0.65 & 0.64 & 0.43 & 26.92 & 29.14 & 24.17 & 109.52 & 312.23 & 7.16 & 202.70 & 446 & 0.25 \\
\hline 0 & 0.44 & 0.45 & 0.45 & 31.47 & 31.47 & 26.36 & 330.14 & 330.14 & 0.00 & 0.00 & 446 & 0.74 \\
\hline 0 & 0.13 & 0.16 & 0.16 & 25.86 & 25.86 & 23.78 & 326.45 & 326.45 & 0.00 & 0.00 & 446 & 0.73 \\
\hline 0 & 0.38 & 0.29 & 0.27 & 27.08 & 26.95 & 23.86 & 281.07 & 291.96 & 0.35 & 10.89 & 446 & 0.63 \\
\hline 0 & 1.68 & 1.46 & 1.33 & 39.67 & 39.56 & 35.17 & 115.04 & 123.16 & 0.21 & 8.12 & 446 & 0.26 \\
\hline 0 & 0.24 & 0.22 & 0.10 & 26.89 & 27.22 & 25.98 & 110.95 & 321.01 & 7.76 & 210.06 & 446 & 0.25 \\
\hline 0 & 0.17 & 0.15 & 0.07 & 29.72 & 29.31 & 28.61 & 210.23 & 307.79 & 3.32 & 97.57 & 446 & 0.47 \\
\hline 0 & 0.13 & 0.11 & 0.11 & 27.11 & 27.11 & 25.70 & 327.67 & 327.67 & 0.00 & 0.00 & 446 & 0.73 \\
\hline 0 & 0.51 & 0.50 & 0.27 & 28.25 & 27.78 & 24.53 & 196.08 & 317.58 & 4.44 & 121.51 & 446 & 0.44 \\
\hline 0 & 0.07 & 0.03 & 0.03 & 25.39 & 25.39 & 24.95 & 346.78 & 346.78 & 0.00 & 0.00 & 446 & 0.78 \\
\hline 0 & 0.59 & 0.61 & 0.30 & 28.25 & 29.03 & 25.98 & 101.81 & 286.76 & 6.45 & 184.95 & 446 & 0.23 \\
\hline 0 & 0.46 & 0.33 & 0.33 & 29.86 & 29.86 & 27.03 & 243.28 & 243.28 & 0.00 & 0.00 & 446 & 0.55 \\
\hline 0 & 0.42 & 0.31 & 0.31 & 31.73 & 31.73 & 30.84 & 90.92 & 90.92 & 0.00 & 0.00 & 446 & 0.20 \\
\hline 0 & 0.48 & 0.37 & 0.37 & 33.89 & 33.89 & 30.75 & 273.29 & 273.29 & 0.00 & 0.00 & 446 & 0.61 \\
\hline 0 & 0.32 & 0.16 & 0.13 & 27.86 & 27.92 & 26.34 & 266.16 & 319.48 & 1.92 & 53.31 & 446 & 0.60 \\
\hline 0 & 0.27 & 0.25 & 0.25 & 28.17 & 28.17 & 26.03 & 230.49 & 230.49 & 0.00 & 0.00 & 446 & 0.52 \\
\hline 0 & 0.46 & 0.51 & 0.51 & 32.31 & 32.31 & 26.48 & 336.12 & 336.12 & 0.00 & 0.00 & 446 & 0.75 \\
\hline 0 & 0.00 & 0.09 & 0.12 & 29.72 & 30.36 & 29.00 & 231.75 & 342.66 & 3.68 & 110.91 & 446 & 0.52 \\
\hline 0 & 0.41 & 0.60 & 0.60 & 30.25 & 30.25 & 23.36 & 305.50 & 305.50 & 0.00 & 0.00 & 446 & 0.68 \\
\hline 0 & 1.09 & 0.92 & 0.45 & 33.53 & 34.72 & 30.42 & 109.21 & 316.46 & 6.09 & 207.25 & 446 & 0.24 \\
\hline 0 & 0.43 & 0.41 & 0.41 & 26.70 & 26.70 & 26.34 & 23.39 & 23.39 & 0.00 & 0.00 & 446 & 0.05 \\
\hline 0 & 0.27 & 0.21 & 0.11 & 29.28 & 29.45 & 28.25 & 138.80 & 300.84 & 5.50 & 162.04 & 446 & 0.31 \\
\hline 0 & 0.19 & 0.17 & 0.16 & 30.70 & 30.70 & 29.06 & 285.30 & 308.00 & 0.75 & 22.70 & 446 & 0.64 \\
\hline 0 & 0.89 & 0.99 & 0.99 & 37.73 & 37.73 & 33.87 & 139.16 & 139.16 & 0.00 & 0.00 & 446 & 0.31 \\
\hline 0 & 0.96 & 0.91 & 0.77 & 25.47 & 26.86 & 21.14 & 109.44 & 178.71 & 2.67 & 69.27 & 446 & 0.25 \\
\hline 1 & 0.51 & 0.39 & 0.15 & 27.61 & 27.75 & 26.09 & 105.45 & 300.23 & 7.01 & 194.78 & 446 & 0.24 \\
\hline 1 & 0.49 & 0.36 & 0.35 & 29.89 & 29.86 & 25.53 & 334.56 & 349.66 & 0.51 & 15.10 & 446 & 0.75 \\
\hline 1 & 0.59 & 0.61 & 0.14 & 25.95 & 25.81 & 23.92 & 82.33 & 346.50 & 10.18 & 264.18 & 446 & 0.18 \\
\hline
\end{tabular}




\begin{tabular}{|c|c|c|c|c|c|c|c|c|c|c|c|c|}
\hline 1 & 0.04 & 0.15 & 0.15 & 28.56 & 28.56 & 26.61 & 360.39 & 360.39 & 0.00 & 0.00 & 446 & 0.81 \\
\hline & 0.43 & 0.37 & 0.28 & 29.50 & 29.68 & 27.12 & 211.31 & 284.83 & 2.53 & 73.52 & 446 & 0.47 \\
\hline & 0.28 & 0.26 & 0.22 & 2.87 & 2.88 & 2.77 & 91.41 & 70.38 & 3.04 & 88.04 & 0 & 0.20 \\
\hline
\end{tabular}


Table 9: Right Lane Average Speeds at Study Sites.

\begin{tabular}{|c|c|c|c|c|c|c|}
\hline $\begin{array}{l}\text { Island Park } \\
\text { Drive (E-N) }\end{array}$ & $\begin{array}{c}\text { Parkdale Ave. } \\
\text { (W-NS) }\end{array}$ & $\begin{array}{c}\text { Bronson Ave. } \\
\text { (W-NS) }\end{array}$ & \begin{tabular}{|c|} 
Moodie Drive \\
(W-NS)
\end{tabular} & $\begin{array}{c}\text { Terry Fox } \\
\text { Drive (E-NS) }\end{array}$ & \begin{tabular}{|c|} 
Carp Road \\
(E-NS)
\end{tabular} & \begin{tabular}{|c|} 
Terry Fox \\
Drive (W-NS)
\end{tabular} \\
\hline 22.93 & 26.81 & 21.20 & 29.28 & 30.58 & 28.12 & 28.89 \\
\hline 24.53 & 29.71 & 27.96 & 20.97 & 26.83 & 28.04 & 28.27 \\
\hline 27.59 & 26.65 & 27.78 & 27.26 & 27.76 & 29.47 & 28.38 \\
\hline 24.92 & 27.71 & 21.55 & 24.82 & 28.42 & 27.87 & 27.58 \\
\hline 27.16 & 26.98 & 21.65 & 29.15 & 26.89 & 29.90 & 27.28 \\
\hline 28.42 & 25.95 & 25.69 & 29.05 & 28.81 & 26.47 & 27.21 \\
\hline 27.54 & 26.46 & 23.62 & 28.50 & 27.58 & 28.91 & 29.65 \\
\hline 23.24 & 22.60 & 26.98 & 28.35 & 25.60 & 27.14 & 29.46 \\
\hline 23.92 & 24.52 & 20.24 & 25.06 & 27.88 & 28.75 & 29.66 \\
\hline 28.53 & 25.28 & 19.70 & 26.60 & 25.20 & 28.57 & 31.85 \\
\hline 27.05 & 20.85 & 26.43 & 26.81 & 28.29 & 27.35 & 26.08 \\
\hline 27.58 & 23.58 & 30.50 & 26.90 & 28.33 & 26.31 & 34.23 \\
\hline 23.41 & 25.29 & 26.71 & 28.58 & 27.94 & 28.22 & 27.46 \\
\hline 25.52 & 28.53 & 26.27 & 25.86 & 31.40 & 29.22 & 33.92 \\
\hline 21.81 & 26.38 & 26.21 & 29.57 & 33.15 & 25.95 & 33.41 \\
\hline 19.55 & 24.16 & 28.99 & 26.83 & 32.43 & 26.26 & 32.18 \\
\hline 24.48 & 28.97 & 24.99 & 28.23 & 28.94 & 28.76 & 31.72 \\
\hline 25.80 & 26.81 & 31.80 & 27.55 & 32.54 & 24.20 & 27.74 \\
\hline 22.76 & 24.13 & 25.92 & 29.68 & 31.19 & 29.12 & 31.03 \\
\hline 22.11 & 20.65 & 26.00 & 33.56 & 27.89 & 27.21 & 30.90 \\
\hline 17.24 & 33.13 & 24.68 & 29.89 & 28.46 & 29.31 & 31.87 \\
\hline 22.05 & 27.77 & 23.45 & 28.32 & 28.71 & 24.88 & 30.99 \\
\hline 24.09 & 22.22 & 25.12 & 29.41 & 31.44 & 29.24 & 36.33 \\
\hline 21.96 & 24.13 & 27.92 & 28.80 & 30.34 & 27.72 & 29.23 \\
\hline 21.20 & 24.62 & 26.30 & 26.75 & 33.22 & 30.90 & 23.92 \\
\hline 20.99 & 31.64 & 28.48 & 26.65 & 28.57 & 25.95 & 31.70 \\
\hline 27.02 & 25.02 & 26.13 & 28.85 & 28.82 & 28.34 & 28.30 \\
\hline 20.77 & 30.63 & 24.09 & 34.83 & 26.29 & 32.54 & 32.09 \\
\hline 20.19 & 30.94 & 27.15 & 28.24 & 26.13 & 32.60 & 29.33 \\
\hline 21.14 & 22.52 & 26.08 & 29.75 & 27.34 & 32.20 & 30.71 \\
\hline 21.04 & 22.43 & 20.21 & 31.25 & 22.65 & 29.42 & 30.44 \\
\hline 22.25 & 29.41 & 31.17 & 26.06 & 26.56 & 32.64 & 32.85 \\
\hline 26.58 & 24.07 & 25.76 & 28.64 & 31.85 & 26.41 & 34.45 \\
\hline 21.65 & 30.15 & 26.23 & 28.81 & 23.66 & 32.94 & 29.83 \\
\hline 25.29 & 22.20 & 26.23 & 31.15 & 29.68 & 30.96 & 26.93 \\
\hline
\end{tabular}




\begin{tabular}{|c|c|c|c|c|c|c|}
\hline 19.45 & 25.68 & 26.57 & 22.65 & 25.15 & 33.50 & 29.56 \\
\hline 21.76 & 23.13 & 27.30 & 24.75 & 32.66 & 31.60 & 32.49 \\
\hline 22.90 & 31.22 & 25.55 & 32.30 & 30.89 & 29.45 & 26.38 \\
\hline 23.07 & 29.47 & 29.23 & 28.41 & 32.14 & 31.79 & 29.27 \\
\hline 20.78 & 24.25 & 25.72 & 28.87 & 29.09 & 27.66 & 30.99 \\
\hline 20.76 & 28.22 & 29.46 & 28.96 & 28.92 & 29.20 & 27.72 \\
\hline 22.80 & 30.73 & 23.17 & 29.90 & 26.57 & 28.93 & 26.13 \\
\hline 21.93 & 28.21 & 29.52 & 28.45 & 28.34 & 28.43 & 30.70 \\
\hline 23.51 & 24.91 & 26.69 & 28.76 & 31.03 & 26.93 & 26.43 \\
\hline 23.11 & 27.53 & 25.53 & 27.73 & 28.17 & 32.09 & 29.52 \\
\hline 21.89 & 27.69 & 30.46 & 28.27 & 29.07 & 31.70 & 31.33 \\
\hline 20.93 & 27.66 & 24.48 & 29.70 & 29.45 & 27.22 & 34.55 \\
\hline 23.66 & 30.31 & 24.61 & 28.87 & 27.55 & 24.78 & 28.36 \\
\hline 23.34 & 26.98 & 26.71 & 26.71 & 30.14 & 29.50 & 30.85 \\
\hline 21.59 & 32.56 & 27.93 & 28.22 & 28.24 & 28.04 & 29.15 \\
\hline 21.57 & 27.07 & 29.72 & 29.59 & 28.25 & 31.77 & 32.32 \\
\hline 25.22 & 28.16 & 25.34 & 30.94 & 33.04 & 27.92 & 30.00 \\
\hline 18.80 & 27.15 & 25.56 & 28.40 & 29.67 & 26.98 & 29.71 \\
\hline 23.56 & 26.28 & 26.42 & 28.32 & 27.16 & 29.47 & 27.82 \\
\hline 21.24 & 27.05 & 24.80 & 30.62 & 29.78 & 29.17 & 28.98 \\
\hline 20.41 & 23.73 & 27.49 & 31.70 & 33.85 & 33.63 & 30.38 \\
\hline 22.45 & 28.66 & 25.61 & 29.07 & 28.80 & 33.41 & 26.24 \\
\hline 22.26 & 29.29 & 27.72 & 29.35 & 27.75 & 32.15 & 27.95 \\
\hline 24.16 & 29.39 & 24.91 & 32.10 & 32.82 & 33.96 & 31.83 \\
\hline 26.91 & 27.90 & 23.55 & 32.32 & 27.30 & 31.14 & 34.47 \\
\hline 27.13 & 24.67 & 26.31 & 29.20 & 28.30 & 32.06 & 26.76 \\
\hline 25.32 & 26.60 & 27.49 & 28.88 & 30.94 & 28.69 & 34.20 \\
\hline 23.33 & 24.40 & 22.98 & 29.43 & 29.04 & 31.13 & 29.26 \\
\hline 18.92 & 25.00 & 24.06 & 25.47 & 30.02 & 30.29 & 29.34 \\
\hline 24.81 & 23.35 & 29.01 & 29.14 & 26.17 & 29.59 & 30.15 \\
\hline 23.73 & 31.68 & 30.38 & 32.79 & 24.77 & 30.75 & 29.49 \\
\hline 24.73 & 21.27 & 26.02 & 30.27 & 30.25 & 32.42 & 31.13 \\
\hline 24.70 & 24.85 & 29.01 & 30.66 & 26.96 & 32.23 & 30.49 \\
\hline 27.11 & 27.94 & 30.20 & 31.39 & 29.58 & 29.86 & 28.10 \\
\hline 24.34 & 29.03 & 27.41 & 29.97 & 27.91 & 29.43 & 27.19 \\
\hline 23.55 & 31.68 & 25.85 & 32.93 & 26.61 & 26.78 & 31.15 \\
\hline 22.96 & 29.78 & 29.26 & 26.45 & 29.51 & 32.55 & 29.06 \\
\hline 22.85 & 32.06 & 31.79 & 26.22 & 29.55 & 30.40 & 30.79 \\
\hline 27.93 & 24.90 & 26.27 & 28.09 & 23.97 & 31.75 & 26.04 \\
\hline
\end{tabular}




\begin{tabular}{|c|c|c|c|c|c|c|}
\hline 28.10 & 28.90 & 27.92 & 30.43 & 32.33 & 28.67 & 33.07 \\
\hline 27.65 & 27.31 & 26.13 & 30.67 & 30.31 & 31.09 & 28.24 \\
\hline 21.74 & 25.20 & 30.85 & 26.69 & 27.63 & 32.24 & 32.05 \\
\hline 20.85 & 26.52 & 23.83 & 29.49 & 27.75 & 27.54 & 27.92 \\
\hline 25.44 & 25.14 & 34.52 & 29.14 & 29.20 & 27.73 & 30.76 \\
\hline 24.35 & 25.38 & 26.08 & 30.30 & 25.02 & 31.87 & 34.23 \\
\hline 26.67 & 28.35 & 24.54 & 28.96 & 30.03 & 25.68 & 27.59 \\
\hline 21.72 & 26.45 & 27.30 & 28.84 & 30.20 & 32.04 & 26.64 \\
\hline 22.43 & 31.42 & 26.81 & 32.04 & 27.42 & 32.53 & 28.36 \\
\hline 30.15 & 28.14 & 26.28 & 28.68 & 28.05 & 30.24 & 31.25 \\
\hline 26.58 & 24.89 & 27.63 & 28.48 & 27.27 & 29.75 & 29.23 \\
\hline 26.98 & 24.73 & 29.75 & 28.53 & 27.93 & 30.95 & 29.20 \\
\hline 26.35 & 25.19 & 28.11 & 29.29 & 26.98 & 27.96 & 29.98 \\
\hline 28.87 & 26.21 & 34.97 & 29.41 & 28.48 & 26.32 & 29.83 \\
\hline 28.00 & 28.35 & 28.56 & 32.17 & 30.53 & 29.35 & 34.32 \\
\hline 27.25 & 25.50 & 22.98 & 25.26 & 29.11 & 33.02 & 30.12 \\
\hline 27.61 & 26.31 & 29.32 & 26.60 & 28.00 & 30.24 & 31.74 \\
\hline 24.48 & 29.88 & 31.34 & 33.03 & 27.23 & 27.73 & 27.54 \\
\hline 24.97 & 22.99 & 27.41 & 26.12 & 29.08 & 30.19 & 29.76 \\
\hline 27.30 & 23.73 & 28.60 & 29.71 & 31.26 & 28.68 & 29.65 \\
\hline 25.75 & 28.85 & 31.29 & 26.35 & 29.51 & 32.42 & 27.56 \\
\hline 24.48 & 25.24 & 27.62 & 28.67 & 29.15 & 32.94 & 32.59 \\
\hline 24.38 & 27.45 & 26.46 & 30.55 & 34.70 & 28.32 & 27.63 \\
\hline 25.02 & 22.71 & 22.79 & 31.05 & 29.08 & 32.63 & 30.11 \\
\hline 29.41 & 23.82 & 34.01 & 30.26 & 26.02 & 24.57 & 28.17 \\
\hline 25.42 & 27.52 & 26.85 & 28.39 & 27.04 & 30.76 & 27.04 \\
\hline 22.44 & 28.40 & 23.51 & 28.66 & 26.77 & 30.44 & 31.97 \\
\hline 24.66 & 29.40 & 32.05 & 33.06 & 29.96 & 33.12 & 30.47 \\
\hline 24.44 & 28.03 & 24.18 & 28.96 & 28.17 & 32.75 & 28.20 \\
\hline 30.98 & 29.12 & 26.44 & 27.49 & 34.06 & 30.61 & 32.54 \\
\hline 26.41 & 26.26 & 29.45 & 35.24 & 25.86 & 34.12 & 23.94 \\
\hline 27.24 & 23.75 & 27.81 & 30.44 & 27.85 & 29.87 & 31.57 \\
\hline 24.57 & 30.14 & 31.02 & 27.36 & 27.99 & 32.58 & 28.39 \\
\hline 30.22 & 29.29 & 22.67 & 31.41 & 31.87 & 34.01 & \\
\hline 26.48 & 26.48 & 25.45 & 27.53 & 28.19 & 34.28 & \\
\hline 29.87 & 36.48 & 20.54 & & 28.39 & 33.40 & \\
\hline 27.51 & 30.92 & 30.78 & & 41.87 & 31.40 & \\
\hline 26.40 & 25.57 & 29.57 & & 32.27 & 26.65 & \\
\hline 24.72 & 30.86 & 26.61 & & 31.00 & 28.08 & \\
\hline
\end{tabular}




\begin{tabular}{|c|c|c|c|c|c|}
\hline 26.20 & 24.60 & 28.16 & 29.62 & 33.75 & \\
\hline 28.20 & 27.83 & 27.13 & 29.46 & 29.07 & \\
\hline 26.55 & 21.33 & 27.81 & 29.55 & 33.56 & \\
\hline 24.68 & 24.13 & 23.44 & 27.77 & 26.50 & \\
\hline 21.19 & 20.18 & 25.16 & 31.79 & 29.87 & \\
\hline 24.41 & 27.10 & 30.97 & 32.84 & 31.75 & \\
\hline 26.88 & 26.97 & & 29.73 & 26.01 & \\
\hline 24.82 & 24.40 & & 26.86 & 28.08 & \\
\hline 25.87 & 27.86 & & 33.39 & 31.03 & \\
\hline 28.57 & 31.47 & & 30.79 & 32.15 & \\
\hline 27.61 & 29.77 & & 33.89 & 28.93 & \\
\hline 26.35 & 26.65 & & & 35.38 & \\
\hline 29.24 & 26.15 & & & & \\
\hline 26.16 & 28.23 & & & & \\
\hline 27.92 & 26.01 & & & & \\
\hline 30.98 & & & & & \\
\hline 28.05 & & & & & \\
\hline 24.58 & & & & & \\
\hline 28.16 & & & & & \\
\hline 20.69 & & & & & \\
\hline 25.54 & & & & & \\
\hline 21.70 & & & & & \\
\hline 25.27 & & & & & \\
\hline 21.63 & & & & & \\
\hline 24.85 & & & & & \\
\hline 26.44 & & & & & \\
\hline 23.85 & & & & & \\
\hline 25.77 & & & & & \\
\hline 24.35 & & & & & \\
\hline 24.78 & & & & & \\
\hline 28.04 & & & & & \\
\hline 26.17 & & & & & \\
\hline 23.81 & & & & & \\
\hline 27.94 & & & & & \\
\hline 25.13 & & & & & \\
\hline 28.65 & & & & & \\
\hline 25.12 & & & & & \\
\hline 26.60 & & & & & \\
\hline 22.42 & & & & & \\
\hline 24.08 & & & & & \\
\hline
\end{tabular}




\begin{tabular}{|l|l|l|l|l|l|l|}
\hline 21.01 & & & & & & \\
\hline \multicolumn{7}{|c|}{ Average } \\
\hline 24.67 & 26.85 & 26.84 & 28.95 & 29.11 & 29.86 & 29.85 \\
\hline
\end{tabular}

Table 10: P-value of Correlation Coefficients at Island Park Drive E-N

\begin{tabular}{|l|c|c|c|r|}
\hline & Initial Speed & Final Speed & Utilization Rate & Deceleration rate \\
\hline Initial Speed & NA & $<0.001$ & $<0.001$ & $<0.001$ \\
\hline Final Speed & $<0.001$ & NA & 0.2067 & 0.0518 \\
\hline Utilization Rate & $<0.001$ & 0.2067 & NA & 0.0143 \\
\hline Deceleration Rate & $<0.001$ & 0.0518 & 0.0143 & NA \\
\hline
\end{tabular}

Table 11: P-value of Correlation Coefficients at Parkdale Avenue W-NS

\begin{tabular}{|l|c|c|c|r|}
\hline & Initial Speed & Final Speed & Utilization Rate & Deceleration rate \\
\hline Initial Speed & NA & $<0.001$ & 0.0086 & $<0.001$ \\
\hline Final Speed & $<0.001$ & NA & $<0.001$ & 0.1000 \\
\hline Utilization Rate & 0.0086 & $<0.001$ & NA & 0.0359 \\
\hline Deceleration Rate & $<0.001$ & 0.1000 & 0.0359 & NA \\
\hline
\end{tabular}

Table 12: P-value of Correlation Coefficients at Bronson Avenue W-NS

\begin{tabular}{|l|c|c|c|c|}
\hline & Initial Speed & Final Speed & Utilization Rate & Deceleration rate \\
\hline Initial Speed & NA & $<0.001$ & 0.0251 & $<0.001$ \\
\hline Final Speed & $<0.001$ & NA & $<0.001$ & 0.0137 \\
\hline Utilization Rate & 0.0251 & $<0.001$ & NA & $<0.001$ \\
\hline Deceleration Rate & $<0.001$ & 0.0137 & $<0.001$ & NA \\
\hline
\end{tabular}

Table 13: P-value of Correlation Coefficients at Moodie Drive W-NS 


\begin{tabular}{|l|c|c|c|c|}
\hline & Initial Speed & Final Speed & Utilization Rate & Deceleration rate \\
\hline Initial Speed & NA & $<0.001$ & 0.3137 & $<0.001$ \\
\hline Final Speed & $<0.001$ & NA & 0.0213 & 0.0316 \\
\hline Utilization Rate & 0.3137 & 0.0213 & NA & $<0.001$ \\
\hline Deceleration Rate & $<0.001$ & 0.0316 & $<0.001$ & NA \\
\hline
\end{tabular}

Table 14: P-value of Correlation Coefficients at Terry Fox Drive E-NS

\begin{tabular}{|l|c|c|c|r|}
\hline & Initial Speed & Final Speed & Utilization Rate & Deceleration rate \\
\hline Initial Speed & NA & $<0.001$ & 0.3490 & $<0.001$ \\
\hline Final Speed & $<0.001$ & NA & $<0.001$ & 0.4741 \\
\hline Utilization Rate & 0.3490 & $<0.001$ & NA & $<0.001$ \\
\hline Deceleration Rate & $<0.001$ & 0.4741 & $<0.001$ & NA \\
\hline
\end{tabular}

Table 15: P-value of Correlation Coefficients at Carp Road E-NS

\begin{tabular}{|l|c|c|c|c|}
\hline & Initial Speed & Final Speed & Utilization Rate & Deceleration rate \\
\hline Initial Speed & NA & $<0.001$ & $<0.001$ & $<0.001$ \\
\hline Final Speed & $<0.001$ & NA & 0.3366 & 0.4791 \\
\hline Utilization Rate & $<0.001$ & 0.3366 & NA & $<0.001$ \\
\hline Deceleration Rate & $<0.001$ & 0.4791 & $<0.001$ & NA \\
\hline
\end{tabular}

Table 16: P-value of Correlation Coefficients at Terry Fox Drive W-NS

\begin{tabular}{|l|c|c|c|c|}
\hline & Initial Speed & Final Speed & Utilization Rate & Deceleration rate \\
\hline Initial Speed & NA & $<0.001$ & 0.3371 & $<0.001$ \\
\hline Final Speed & $<0.001$ & NA & 0.0039 & $<0.001$ \\
\hline Utilization Rate & 0.3371 & 0.0039 & NA & $<0.001$ \\
\hline Deceleration Rate & $<0.001$ & $<0.001$ & $<0.001$ & NA \\
\hline
\end{tabular}


Table 17: P-value of Correlation Coefficients of All Sites Combined Dataset W-

\begin{tabular}{|l|c|c|c|c|}
\hline & Initial Speed & Final Speed & Utilization Rate & Deceleration rate \\
\hline Initial Speed & NA & $<0.001$ & 0.4287 & $<0.001$ \\
\hline Final Speed & $<0.001$ & NA & 0.1116 & $<0.001$ \\
\hline Utilization Rate & 0.4287 & 0.1116 & NA & $<0.001$ \\
\hline Deceleration Rate & $<0.001$ & $<0.001$ & $<0.001$ & NA \\
\hline
\end{tabular}




\section{Appendix 4: Initial Speeds ANOVA Test at Study}

Sites. 
Anova: Single Factor

ALL SCLS

SUMMARY

\begin{tabular}{lrcrr}
\hline \multicolumn{1}{c}{ Groups } & \multicolumn{1}{c}{ Count } & Sum & Average & Variance \\
\hline Island Park Drive (E-N) & 85.000 & 1748.977 & 20.576 & 4.675 \\
Parkdale Ave. (W-NS) & 90.000 & 2096.289 & 23.292 & 5.504 \\
Bronson Ave. (W-NS) & 90.000 & 2176.938 & 24.188 & 5.067 \\
Moodie Drive (W-NS) & 85.000 & 2412.660 & 28.384 & 6.630 \\
Terry Fox Drive (E-NS) & 114.000 & 3267.331 & 28.661 & 5.716 \\
Carp Road (E-NS) & 98.000 & 2867.162 & 29.257 & 5.862 \\
Terry Fox Drive (W-NS) & 76.000 & 2241.900 & 29.499 & 8.264 \\
\hline
\end{tabular}

ANOVA

\begin{tabular}{lrrrrrr}
\hline \multicolumn{1}{c}{ Source of Variation } & \multicolumn{1}{c}{ SS } & \multicolumn{1}{c}{$d f$} & \multicolumn{1}{c}{ MS } & $F$ & p-value & F crit \\
\hline Between Groups & 6637.774 & 6.000 & 1106.296 & 187.414 & $<0.001$ & 2.113 \\
Within Groups & 3724.757 & 631.000 & 5.903 & & & \\
& & & & & & \\
Total & 10362.531 & 637.000 & & & & \\
\hline
\end{tabular}

Anova: Single Factor

Remove Island Park Drive (E-N)

SUMMARY

\begin{tabular}{crcrr}
\hline Groups & \multicolumn{1}{c}{ Count } & \multicolumn{1}{c}{ Sum } & Average & Variance \\
\hline Parkdale Ave. (W-NS) & 90.000 & 2096.289 & 23.292 & 5.504 \\
Bronson Ave. (W-NS) & 90.000 & 2176.938 & 24.188 & 5.067 \\
Moodie Drive (W-NS) & 85.000 & 2412.660 & 28.384 & 6.630 \\
Terry Fox Drive (E-NS) & 114.000 & 3267.331 & 28.661 & 5.716 \\
Carp Road (E-NS) & 98.000 & 2867.162 & 29.257 & 5.862 \\
Terry Fox Drive (W-NS) & 76.000 & 2241.900 & 29.499 & 8.264 \\
\hline
\end{tabular}

\begin{tabular}{lcrrrrr} 
ANOVA & \multicolumn{1}{c}{ Df } & \multicolumn{1}{c}{ MS } & \multicolumn{1}{c}{$F$} & p-value & F crit \\
\hline Source of Variation & SS & \multicolumn{1}{c}{ Df } & & & \\
Between Groups & 3368.676 & 5.000 & 673.735 & 110.602 & $<0.001$ & 2.230 \\
Within Groups & 3332.066 & 547.000 & 6.092 & & & \\
& & & & & & \\
Total & 6700.742 & 552.000 & & & & \\
\hline
\end{tabular}


Anova: Single Factor

Remove Parkdale Ave.

(W-NS)

SUMMARY

\begin{tabular}{crcrr}
\hline Groups & \multicolumn{1}{c}{ Count } & \multicolumn{1}{c}{ Sum } & Average & Variance \\
\hline Bronson Ave. (W-NS) & 90.000 & 2176.938 & 24.188 & 5.067 \\
Moodie Drive (W-NS) & 85.000 & 2412.660 & 28.384 & 6.630 \\
Terry Fox Drive (E-NS) & 114.000 & 3267.331 & 28.661 & 5.716 \\
Carp Road (E-NS) & 98.000 & 2867.162 & 29.257 & 5.862 \\
Terry Fox Drive (W-NS) & 76.000 & 2241.900 & 29.499 & 8.264 \\
\hline
\end{tabular}

ANOVA

\begin{tabular}{lrrrrrr}
\hline Source of Variation & \multicolumn{1}{c}{ SS } & \multicolumn{1}{c}{$d f$} & \multicolumn{1}{c}{ MS } & \multicolumn{1}{c}{$F$} & $p$-value & F crit \\
\hline Between Groups & 1695.489 & 4.000 & 423.872 & 68.304 & $<0.001$ & 2.391 \\
Within Groups & 2842.190 & 458.000 & 6.206 & & & \\
& & & & & & \\
Total & 4537.679 & 462.000 & & & & \\
\hline
\end{tabular}

Remove Bronson

Anova: Single Factor

Ave. (W-NS)

SUMMARY

\begin{tabular}{crcrr}
\hline Groups & \multicolumn{1}{c}{ Count } & \multicolumn{1}{c}{ Sum } & Average & Variance \\
\hline Moodie Drive (W-NS) & 85.000 & 2412.660 & 28.384 & 6.630 \\
Terry Fox Drive (E-NS) & 114.000 & 3267.331 & 28.661 & 5.716 \\
Carp Road (E-NS) & 98.000 & 2867.162 & 29.257 & 5.862 \\
Terry Fox Drive (W-NS) & 76.000 & 2241.900 & 29.499 & 8.264 \\
\hline
\end{tabular}

ANOVA

\begin{tabular}{lrrrrrr}
\hline Source of Variation & \multicolumn{1}{c}{ SS } & \multicolumn{1}{c}{$d f$} & \multicolumn{1}{c}{ MS } & \multicolumn{1}{c}{ F } & p-value & F crit \\
\hline Between Groups & 68.612 & 3.000 & 22.871 & 3.529 & 0.015 & 2.629 \\
Within Groups & 2391.265 & 369.000 & 6.480 & & & \\
& & & & & & \\
Total & 2459.877 & 372.000 & & & & \\
\hline
\end{tabular}


Remove Moodie

Anova: Single Factor

Drive (W-NS)

SUMMARY

\begin{tabular}{lrrrr}
\hline \multicolumn{1}{c}{ Groups } & Count & Sum & Average & Variance \\
\hline Terry Fox Drive (E- & & & & \\
NS) & 114 & 3267.331 & 28.661 & 5.716 \\
Carp Road (E-NS) & 98 & 2867.162 & 29.2568 & 5.862 \\
$\begin{array}{l}\text { Terry Fox Drive } \\
\text { (W-NS) }\end{array}$ & 76 & 2241.9 & 29.499 & 8.264 \\
\hline
\end{tabular}

ANOVA

\begin{tabular}{lrrrrrr}
\hline \multicolumn{1}{c}{$\begin{array}{l}\text { Source of } \\
\text { Variation }\end{array}$} & \multicolumn{1}{c}{ SS } & Df & \multicolumn{1}{c}{ MS } & \multicolumn{1}{c}{ F } & p-value & Fcrit \\
\hline Between Groups & 36.411 & 2 & 18.205 & 2.828557 & $\mathbf{0 . 0 6 1}$ & 3.027 \\
Within Groups & 1834.347 & 285 & 6.436 & & & \\
& & & & & & \\
Total & 1870.758 & 287 & & & \\
\hline
\end{tabular}




\section{Appendix 5: FRL and Diverge Speeds ANOVA Test at Each Site.}


Anova: Single

Factor

Island Park Drive (E-N)

SUMMARY

\begin{tabular}{lrrrr}
\hline \multicolumn{1}{c}{ Groups } & Count & Sum & Average & Variance \\
\hline Diverge Speed & 85.00 & 1748.98 & 20.58 & 4.67 \\
RL Speed & 154.00 & 3799.92 & 24.67 & 7.52 \\
\hline
\end{tabular}

ANOVA

\begin{tabular}{lrrrrrr}
\hline \multicolumn{1}{c}{$\begin{array}{l}\text { Source of } \\
\text { Variation }\end{array}$} & \multicolumn{1}{c}{ SS } & \multicolumn{1}{c}{ Df } & \multicolumn{1}{c}{ MS } & \multicolumn{1}{c}{ F } & p-value & F crit \\
\hline Between Groups & 920.06 & 1.00 & 920.06 & 141.35 & $<0.001$ & 3.88 \\
Within Groups & 1542.66 & 237.00 & 6.51 & & & \\
& & & & & & \\
Total & 2462.72 & 238.00 & & & & \\
\hline
\end{tabular}

Anova: Single

Factor

Parkdale Ave.

(W-NS)

SUMMARY

\begin{tabular}{lrrrr}
\hline \multicolumn{1}{c}{ Groups } & \multicolumn{1}{c}{ Count } & \multicolumn{1}{c}{ Sum } & Average & \multicolumn{2}{c}{ Variance } \\
\hline Diverge Speed & 90.00 & 2094.91 & 23.28 & 5.47 \\
RL Speed & 128.00 & 3436.87 & 26.85 & 8.51 \\
\hline
\end{tabular}

\begin{tabular}{lrrrrrrr} 
ANOVA & \multicolumn{1}{c}{ MS } & F & & & \\
\hline \multicolumn{1}{c}{$\begin{array}{l}\text { Source of } \\
\text { Variation }\end{array}$} & \multicolumn{1}{c}{ SS } & \multicolumn{1}{c}{ Df } & \multicolumn{1}{c}{ MS } & F crit \\
\hline Between Groups & 674.89 & 1.00 & 674.89 & & 93.04 & $<0.001$ & 3.88 \\
Within Groups & 1566.84 & 216.00 & 7.25 & & & & \\
& & & & & & \\
Total & 2241.73 & 217.00 & & & & \\
\hline
\end{tabular}


Anova: Single

Factor

Bronson Ave.

(W-NS)

SUMMARY

\begin{tabular}{lrrrr}
\hline \multicolumn{1}{c}{ Groups } & \multicolumn{1}{c}{ Count } & \multicolumn{1}{c}{ Sum } & Average & \multicolumn{2}{c}{ Variance } \\
\hline Diverge Speed & 90.00 & 2182.82 & 24.25 & 5.20 \\
RL Speed & 119.00 & 3194.01 & 26.84 & 8.63 \\
\hline
\end{tabular}

ANOVA

\begin{tabular}{lrrrrrrr}
\hline \multicolumn{1}{c}{$\begin{array}{l}\text { Source of } \\
\text { Variation }\end{array}$} & \multicolumn{1}{c}{ SS } & \multicolumn{1}{c}{$d f$} & \multicolumn{1}{c}{ MS } & $F$ & & p-value & F crit \\
\hline Between Groups & 342.91 & 1.00 & 342.91 & & 47.92 & $<0.001$ & 3.89 \\
Within Groups & 1481.17 & 207.00 & 7.16 & & & & \\
& & & & & & \\
Total & 1824.07 & 208.00 & & & & \\
\hline
\end{tabular}

Anova: Single

Factor

Moodie Drive

(W-NS)

SUMMARY

\begin{tabular}{lrrrr}
\hline \multicolumn{1}{c}{ Groups } & \multicolumn{1}{c}{ Count } & Sum & Average & Variance \\
\hline Diverge Speed & 85.00 & 2410.26 & 28.36 & 6.20 \\
RL Speed & 109.00 & 3155.14 & 28.95 & 5.25 \\
\hline
\end{tabular}

ANOVA

\begin{tabular}{lrrrrrrr}
\hline \multicolumn{1}{c}{$\begin{array}{l}\text { Source of } \\
\text { Variation }\end{array}$} & \multicolumn{1}{c}{ SS } & \multicolumn{1}{c}{$d f$} & \multicolumn{1}{c}{ MS } & $F$ & & p-value & F crit \\
\hline Between Groups & 16.64 & 1.00 & 16.64 & & 2.93 & $\mathbf{0 . 0 9}$ & 3.89 \\
Within Groups & 1088.53 & 192.00 & 5.67 & & & & \\
& & & & & & & \\
Total & 1105.17 & 193.00 & & & & & \\
\hline
\end{tabular}


Anova: Single Factor

Terry Fox Drive (E-

NS)

SUMMARY

\begin{tabular}{lccrr}
\hline \multicolumn{1}{c}{ Groups } & Count & Sum & Average & \multicolumn{2}{c}{ Variance } \\
\hline Diverge Speed & 114.00 & 3301.99 & 28.96 & \\
RL Speed & 124.00 & 3610.00 & 29.11 & 4.98 \\
\hline
\end{tabular}

ANOVA

\begin{tabular}{lrrrrrrr}
\hline \multicolumn{1}{c}{$\begin{array}{c}\text { Source of } \\
\text { Variation }\end{array}$} & \multicolumn{1}{c}{ SS } & \multicolumn{1}{c}{$d f$} & MS & F & & p-value & Fcrit \\
\hline Between & & & & & 0.22 & $\mathbf{0 . 6 4}$ & 3.88 \\
Groups & 1.30 & 1.00 & 1.30 & & & & \\
Within Groups & 1392.10 & 236.00 & 5.90 & & & \\
Total & 1393.40 & 237.00 & & & & \\
\hline
\end{tabular}

Anova: Single

Factor

SUMMARY

\begin{tabular}{lrrrr}
\hline \multicolumn{1}{c}{ Groups } & \multicolumn{1}{c}{ Count } & Sum & Average & \multicolumn{2}{c}{ Variance } \\
\hline Diverge Speed & 98.00 & 2899.47 & 29.59 & 5.46 \\
RL Speed & 125.00 & 3733.07 & 29.86 & 6.17 \\
\hline
\end{tabular}

ANOVA

\begin{tabular}{lrrrrrrr}
\hline $\begin{array}{l}\text { Source of } \\
\text { Variation }\end{array}$ & \multicolumn{1}{c}{ SS } & \multicolumn{1}{c}{ Df } & MS & $F$ & & p-value & Fcrit \\
\hline Between Groups & 4.25 & 1.00 & 4.25 & & 0.73 & $\mathbf{0 . 4 0}$ & 3.88 \\
Within Groups & 1294.51 & 221.00 & 5.86 & & & & \\
& & & & & & \\
Total & 1298.76 & 222.00 & & & & \\
\hline
\end{tabular}


Anova: Single

Factor

Terry Fox

Drive (W-NS)

SUMMARY

\begin{tabular}{lrrrr}
\hline \multicolumn{1}{c}{ Groups } & \multicolumn{1}{c}{ Count } & Sum & Average & \multicolumn{2}{c}{ Variance } \\
\hline Diverge Speed & 76.00 & 2255.54 & 29.68 & 8.31 \\
RL Speed & 107.00 & 3193.81 & 29.85 & 5.78 \\
\hline
\end{tabular}

ANOVA

\begin{tabular}{lrrrrrrr}
\hline \multicolumn{1}{c}{$\begin{array}{l}\text { Source of } \\
\text { Variation }\end{array}$} & \multicolumn{1}{c}{ SS } & \multicolumn{1}{c}{$d f$} & MS & $F$ & & p-value & Fcrit \\
\hline $\begin{array}{l}\text { Between } \\
\text { Groups }\end{array}$ & 1.29 & 1.00 & 1.29 & & 0.19 & $\mathbf{0 . 6 6}$ & 3.89 \\
Within Groups & 1236.50 & 181.00 & 6.83 & & & & \\
& & & & & & \\
Total & 1237.80 & 182.00 & & & & \\
\hline
\end{tabular}




\section{Appendix 6: Multiple Linear Regression of Deceleration Used in MCS.}


Table 18: Deceleration Regression at Island Park Drive (E-N).

\begin{tabular}{|c|c|c|c|c|c|c|c|c|}
\hline \multicolumn{2}{|c|}{ Regression Statistics } & & & & & & & \\
\hline Multiple R & 0.970 & & & & & & & \\
\hline R Square & 0.940 & & & & & & & \\
\hline Adjusted R & & & & & & & & \\
\hline Square & 0.938 & & & & & & & \\
\hline Standard & & & & & & & & \\
\hline Error & 0.071 & & & & & & & \\
\hline Observatio & & & & & & & & \\
\hline ns & 85.000 & & & & & & & \\
\hline \multicolumn{9}{|l|}{ ANOVA } \\
\hline & & \multicolumn{7}{|c|}{ Significance } \\
\hline & $d f$ & SS & MS & $F$ & $F$ & & & \\
\hline Regression & 3.000 & 6.478 & 2.159 & 423.390 & 0.000 & & & \\
\hline Residual & 81.000 & 0.413 & 0.005 & & & & & \\
\hline \multirow[t]{3}{*}{ Total } & 84.000 & 6.891 & & & & & & \\
\hline & & Standard & & & Lower & Upper & Lower & Upper \\
\hline & Coefficients & Error & t Stat & $p$-value & $95 \%$ & $95 \%$ & $95.0 \%$ & $95.0 \%$ \\
\hline $\begin{array}{l}\text { Intercept } \\
\text { Initial }\end{array}$ & 0.040 & 0.076 & 0.527 & 0.599 & -0.112 & 0.192 & -0.112 & 0.192 \\
\hline Speed & 0.557 & 0.016 & 35.410 & $<0.001$ & 0.525 & 0.588 & 0.525 & 0.588 \\
\hline $\begin{array}{l}\text { Final Speed } \\
\text { Utilization }\end{array}$ & -0.530 & 0.015 & -35.014 & $<0.001$ & -0.560 & -0.499 & -0.560 & -0.499 \\
\hline Rate & -0.944 & 0.060 & -15.764 & $<0.001$ & -1.063 & -0.825 & -1.063 & -0.825 \\
\hline
\end{tabular}


Table 19: Deceleration Regression at Parkdale Ave. (W-NS).

\begin{tabular}{|c|c|c|c|c|c|c|c|c|}
\hline \multicolumn{2}{|c|}{ Regression Statistics } & & & & & & & \\
\hline Multiple R & 0.981 & & & & & & & \\
\hline $\begin{array}{l}\text { R Square } \\
\text { Adjusted R }\end{array}$ & 0.963 & & & & & & & \\
\hline $\begin{array}{l}\text { Square } \\
\text { Standard }\end{array}$ & 0.961 & & & & & & & \\
\hline Error & 0.070 & & & & & & & \\
\hline Observatio & & & & & & & & \\
\hline ns & 90.000 & & & & & & & \\
\hline \multicolumn{9}{|l|}{ ANOVA } \\
\hline & & \multicolumn{7}{|c|}{ Significance } \\
\hline & $d f$ & SS & $M S$ & $F$ & $F$ & & & \\
\hline Regression & 3.000 & 10.977 & 3.659 & 736.389 & 0.000 & & & \\
\hline Residual & 86.000 & 0.427 & 0.005 & & & & & \\
\hline \multirow[t]{3}{*}{ Total } & 89.000 & 11.404 & & & & & & \\
\hline & & Standard & & & Lower & Upper & Lower & Upper \\
\hline & Coefficients & Error & t Stat & $p$-value & $95 \%$ & $95 \%$ & $95.0 \%$ & $95.0 \%$ \\
\hline $\begin{array}{l}\text { Intercept } \\
\text { Initial }\end{array}$ & 0.046 & 0.089 & 0.510 & 0.611 & -0.132 & 0.223 & -0.132 & 0.223 \\
\hline Speed & 0.313 & 0.007 & 46.858 & $<0.001$ & 0.299 & 0.326 & 0.299 & 0.326 \\
\hline $\begin{array}{l}\text { Final Speed } \\
\text { Utilization }\end{array}$ & -0.273 & 0.007 & -40.472 & $<0.001$ & -0.286 & -0.259 & -0.286 & -0.259 \\
\hline Rate & -2.215 & 0.105 & -21.185 & $<0.001$ & -2.423 & -2.007 & -2.423 & -2.007 \\
\hline
\end{tabular}


Table 20: Deceleration Regression atBronson Ave. (W-NS).

\begin{tabular}{lr}
\hline \multicolumn{2}{c}{ Regression Statistics } \\
\hline Multiple R & 0.963 \\
R Square & 0.927 \\
Adjusted R & \\
Square & 0.924 \\
Standard & \\
Error & 0.108 \\
Observation & \\
s & 90.000 \\
\hline
\end{tabular}

ANOVA

\begin{tabular}{lrrrcc}
\hline & & & & \multicolumn{3}{r}{ Significance } \\
& \multicolumn{1}{c}{$d f$} & \multicolumn{1}{c}{ SS } & MS & \multicolumn{1}{c}{$F$} & \multicolumn{1}{c}{$F$} \\
\hline Regression & 3.000 & 12.709 & 4.236 & 362.307 & 0.000 \\
Residual & 86.000 & 1.006 & 0.012 & & \\
Total & 89.000 & 13.715 & & & \\
\hline
\end{tabular}

\begin{tabular}{lrccccccc}
\hline & \multicolumn{3}{c}{ Standard } & & Lower & Upper & Lower & Upper \\
& Coefficients & Error & t Stat & p-value & $95 \%$ & $95 \%$ & $95.0 \%$ & $95.0 \%$ \\
\hline Intercept & -0.289 & 0.142 & -2.040 & 0.044 & -0.571 & -0.007 & -0.571 & -0.007 \\
Initial Speed & 0.225 & 0.007 & 31.127 & $<0.001$ & 0.211 & 0.240 & 0.211 & 0.240 \\
Final Speed & -0.162 & 0.006 & -26.147 & $<0.001$ & -0.175 & -0.150 & -0.175 & -0.150 \\
Utilization & & & & & & & & \\
Rate & -2.024 & 0.129 & -15.709 & $<0.001$ & -2.280 & -1.768 & -2.280 & -1.768 \\
\hline
\end{tabular}


Table 21: Deceleration Regression at Moodie Drive (W-NS).

\begin{tabular}{|c|c|c|c|c|c|c|c|c|}
\hline \multicolumn{2}{|c|}{ Regression Statistics } & & & & & & & \\
\hline Multiple R & 0.964 & & & & & & & \\
\hline R Square & 0.929 & & & & & & & \\
\hline Adjusted & & & & & & & & \\
\hline R Square & 0.926 & & & & & & & \\
\hline Standard & & & & & & & & \\
\hline Error & 0.048 & & & & & & & \\
\hline Observati & & & & & & & & \\
\hline ons & 85.000 & & & & & & & \\
\hline \multicolumn{9}{|l|}{ ANOVA } \\
\hline & & \multicolumn{7}{|c|}{ Significance } \\
\hline & $d f$ & SS & MS & $F$ & $F$ & & & \\
\hline \multicolumn{9}{|l|}{ Regressio } \\
\hline $\mathrm{n}$ & 3.000 & 2.385 & 0.795 & 351.897 & 0.000 & & & \\
\hline Residual & 81.000 & 0.183 & 0.002 & & & & & \\
\hline \multirow[t]{4}{*}{ Total } & 84.000 & 2.568 & & & & & & \\
\hline & & Standa & & & & & & \\
\hline & & $r d$ & & & Lower & Upper & Lower & Upper \\
\hline & Coefficients & Error & t Stat & $p-v a l u e$ & $95 \%$ & $95 \%$ & $95.0 \%$ & $95.0 \%$ \\
\hline $\begin{array}{l}\text { Intercept } \\
\text { Initial }\end{array}$ & 0.060 & 0.061 & 0.984 & 0.328 & -0.061 & 0.180 & -0.061 & 0.180 \\
\hline $\begin{array}{l}\text { Speed } \\
\text { Final }\end{array}$ & 0.184 & 0.006 & 29.793 & $<0.001$ & 0.172 & 0.197 & 0.172 & 0.197 \\
\hline $\begin{array}{l}\text { Speed } \\
\text { Utilization }\end{array}$ & -0.173 & 0.006 & -27.695 & $<0.001$ & -0.185 & -0.160 & -0.185 & -0.160 \\
\hline Rate & -0.693 & 0.039 & -17.651 & $<0.001$ & -0.772 & -0.615 & -0.772 & -0.615 \\
\hline
\end{tabular}


Table 22: Deceleration Regression at Terry Fox Drive (E-NS).

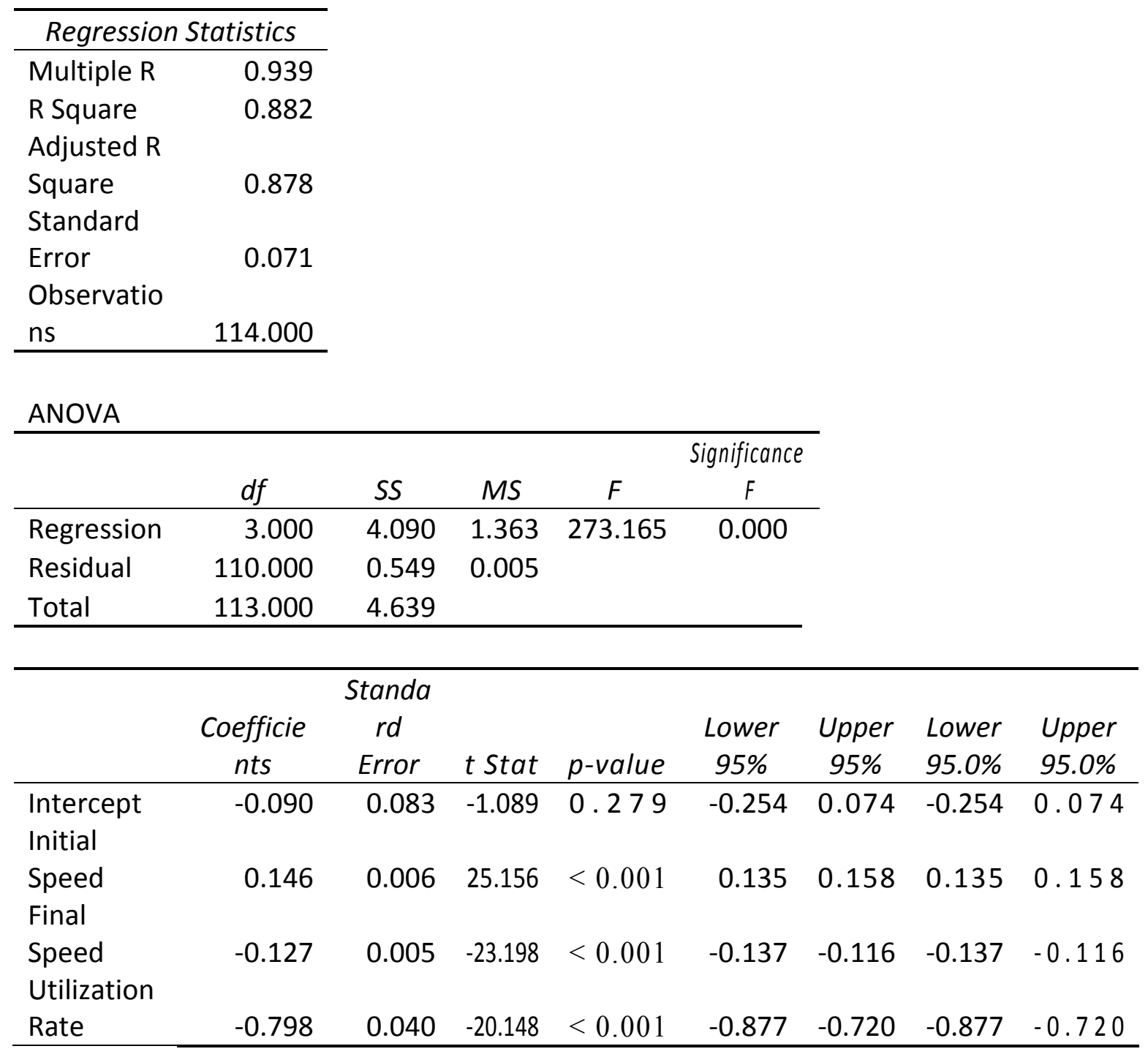


Table 23: Deceleration Regression at Carp Road (E-NS).

\begin{tabular}{lr}
\hline \multicolumn{2}{c}{ Regression Statistics } \\
\hline Multiple R & 0.937 \\
R Square & 0.878 \\
$\begin{array}{l}\text { Adjusted R } \\
\text { Square }\end{array}$ & 0.874 \\
$\begin{array}{l}\text { Standard } \\
\text { Error }\end{array}$ & 0.069 \\
Observatio & \\
ns & 98.000 \\
\hline
\end{tabular}

ANOVA

\begin{tabular}{|c|c|c|c|c|c|c|c|c|}
\hline & & & & & Significance & & & \\
\hline & $d f$ & SS & $M S$ & $F$ & $F$ & & & \\
\hline Regression & 3.000 & 3.164 & 1.055 & 224.718 & 0.000 & & & \\
\hline Residual & 94.000 & 0.441 & 0.005 & & & & & \\
\hline \multirow[t]{2}{*}{ Total } & 97.000 & 3.605 & & & & & & \\
\hline & $\begin{array}{c}\text { Coefficien } \\
\text { ts }\end{array}$ & $\begin{array}{c}\text { Standar } \\
d \text { Error }\end{array}$ & t Stat & $p$-value & $\begin{array}{c}\text { Lower } \\
95 \%\end{array}$ & $\begin{array}{c}\text { Upper } \\
95 \%\end{array}$ & $\begin{array}{l}\text { Lower } \\
95.0 \% \\
\end{array}$ & $\begin{array}{l}\text { Upper } \\
95.0 \% \\
\end{array}$ \\
\hline $\begin{array}{l}\text { Intercept } \\
\text { Initial }\end{array}$ & 0.104 & 0.087 & 1.196 & 0.235 & -0.069 & 0.278 & -0.069 & 0.278 \\
\hline $\begin{array}{l}\text { Speed } \\
\text { Final }\end{array}$ & 0.128 & 0.006 & 21.692 & $<0.001$ & 0.116 & 0.140 & 0.116 & 0.140 \\
\hline $\begin{array}{l}\text { Speed } \\
\text { Utilization }\end{array}$ & -0.118 & 0.006 & -18.800 & $<0.001$ & -0.131 & -0.106 & -0.131 & -0.106 \\
\hline Rate & -0.639 & 0.033 & -19.486 & $<0.001$ & -0.704 & -0.574 & -0.704 & -0.574 \\
\hline
\end{tabular}


Table 24: Deceleration Regression at Terry Fox Drive (W-NS)

\begin{tabular}{|c|c|c|c|c|c|c|c|c|}
\hline \multicolumn{2}{|c|}{ Regression Statistics } & & & & & & & \\
\hline Multiple R & 0.918 & & & & & & & \\
\hline R Square & 0.843 & & & & & & & \\
\hline \multicolumn{9}{|l|}{ Adjusted R } \\
\hline Square & 0.837 & & & & & & & \\
\hline \multicolumn{9}{|l|}{ Standard } \\
\hline Error & 0.103 & & & & & & & \\
\hline \multicolumn{9}{|l|}{ Observatio } \\
\hline ns & 76.000 & & & & & & & \\
\hline \multicolumn{9}{|l|}{ ANOVA } \\
\hline \multicolumn{9}{|c|}{ Significance } \\
\hline & $d f$ & SS & $M S$ & $F$ & $F$ & & & \\
\hline \multicolumn{9}{|c|}{128.99} \\
\hline Regression & 3.000 & 4.141 & 1.380 & 4 & 0.000 & & & \\
\hline Residual & 72.000 & 0.770 & 0.011 & & & & & \\
\hline \multirow[t]{2}{*}{ Total } & 75.000 & 4.911 & & & & & & \\
\hline & $\begin{array}{c}\text { Coefficien } \\
\text { ts }\end{array}$ & $\begin{array}{l}\text { Standar } \\
d \text { Error }\end{array}$ & $t$ Stat & $p$-value & $\begin{array}{c}\text { Lower } \\
95 \%\end{array}$ & $\begin{array}{c}\text { Upper } \\
95 \%\end{array}$ & $\begin{array}{l}\text { Lower } \\
95.0 \%\end{array}$ & $\begin{array}{l}\text { Upper } \\
95.0 \%\end{array}$ \\
\hline $\begin{array}{l}\text { Intercept } \\
\text { Initial }\end{array}$ & -0.180 & 0.128 & -1.398 & 0.166 & -0.436 & 0.076 & -0.436 & 0.076 \\
\hline Speed & 0.152 & 0.009 & 16.843 & $<0.001$ & 0.134 & 0.170 & 0.134 & 0.170 \\
\hline $\begin{array}{l}\text { Final Speed } \\
\text { Utilization }\end{array}$ & -0.131 & 0.009 & -13.938 & $<0.001$ & -0.150 & -0.112 & -0.150 & -0.112 \\
\hline Rate & -0.801 & 0.060 & -13.288 & $<0.001$ & -0.921 & -0.681 & -0.921 & -0.681 \\
\hline
\end{tabular}


Table 25: Deceleration Regression Using All Sites Combined Data

SUMMARY OUTPUT

\begin{tabular}{lr}
\hline \multicolumn{2}{c}{ Regression Statistics } \\
\hline Multiple R & 0.922 \\
R Square & 0.849 \\
Adjusted R & \\
Square & 0.848 \\
Standard & \\
Error & 0.150 \\
Observatio & \\
ns & 638.000 \\
\hline
\end{tabular}

ANOVA

\begin{tabular}{|c|c|c|c|c|c|c|c|c|}
\hline & \multirow[b]{2}{*}{$d f$} & \multirow[b]{2}{*}{ SS } & \multicolumn{3}{|r|}{ Significance } & & & \\
\hline & & & $M S$ & $F$ & F & & & \\
\hline Regression & 4.000 & 80.690 & 20.173 & 891.270 & 0.000 & & & \\
\hline Residual & 633.000 & 14.327 & 0.023 & & & & & \\
\hline \multirow[t]{2}{*}{ Total } & 637.000 & 95.017 & & & & & & \\
\hline & $\begin{array}{c}\text { Coefficien } \\
\text { ts }\end{array}$ & $\begin{array}{l}\text { Standa } \\
\text { rd Error }\end{array}$ & t Stat & $p$-value & $\begin{array}{c}\text { Lower } \\
95 \%\end{array}$ & $\begin{array}{c}\text { Upper } \\
95 \%\end{array}$ & $\begin{array}{l}\text { Lower } \\
95.0 \%\end{array}$ & $\begin{array}{l}\text { Upper } \\
95.0 \%\end{array}$ \\
\hline $\begin{array}{l}\text { Intercept } \\
\text { Initial }\end{array}$ & 0.547 & 0.051 & 10.773 & $<0.001$ & 0.447 & 0.646 & 0.447 & 0.646 \\
\hline $\begin{array}{l}\text { Speed } \\
\text { Final }\end{array}$ & 0.189 & 0.004 & 46.760 & $<0.001$ & 0.181 & 0.197 & 0.181 & 0.197 \\
\hline $\begin{array}{l}\text { Speed } \\
\text { Utilization }\end{array}$ & -0.169 & 0.003 & -48.542 & $<0.001$ & -0.176 & -0.163 & -0.176 & -0.163 \\
\hline Rate & -0.835 & 0.036 & -23.070 & $<0.001$ & -0.906 & -0.764 & -0.906 & -0.764 \\
\hline SCL Length & -0.002 & 0.000 & -25.622 & $<0.001$ & -0.002 & -0.002 & -0.002 & -0.002 \\
\hline
\end{tabular}




\section{Appendix 7: Diagnostics of the Regression Deceleration Rates Used in MCS.}




\section{Island Park Drive (E-N)}

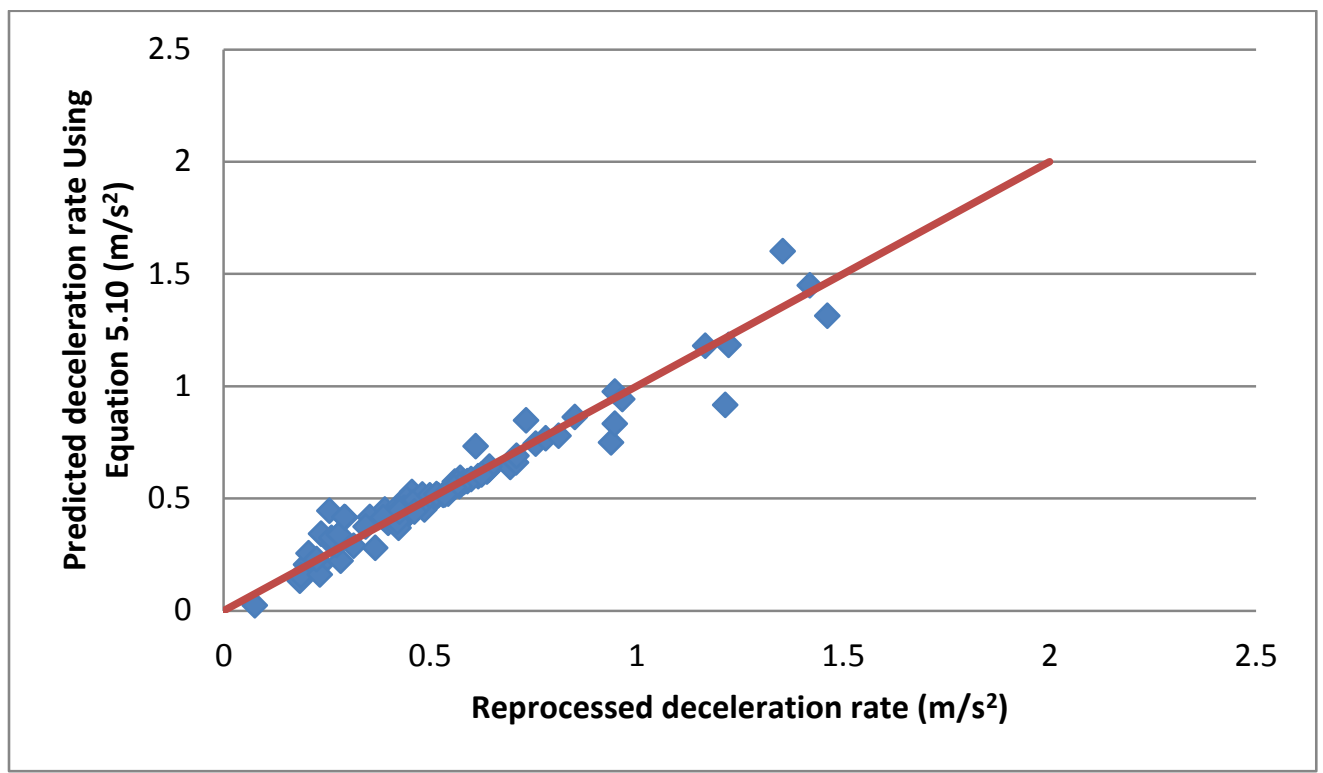

Figure 1: Line of Equality between Reprocessed Deceleration Rate and Predicted Deceleration Rate Using Equation 5.10.

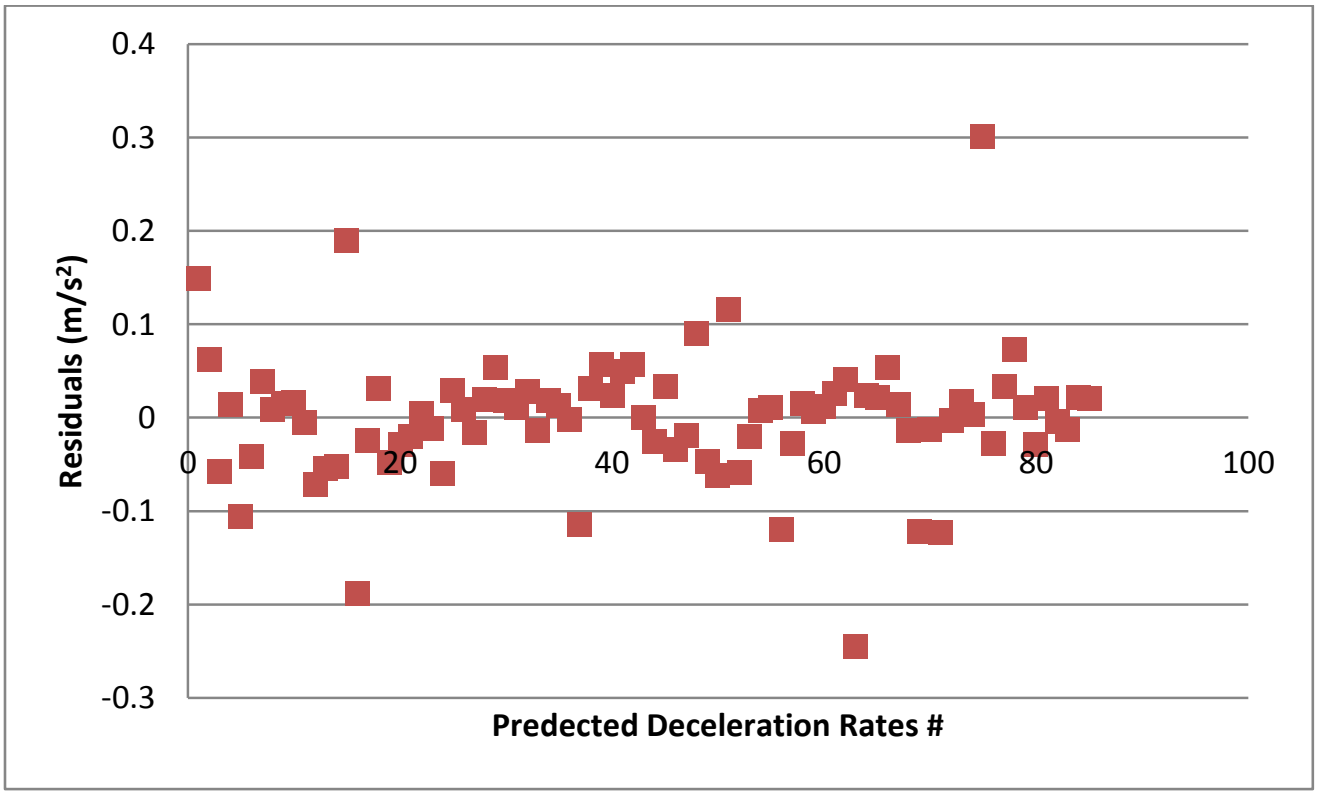

Figure 2: Residuals Plot for Each Predicted Deceleration Rate Using Equation 5.10. 


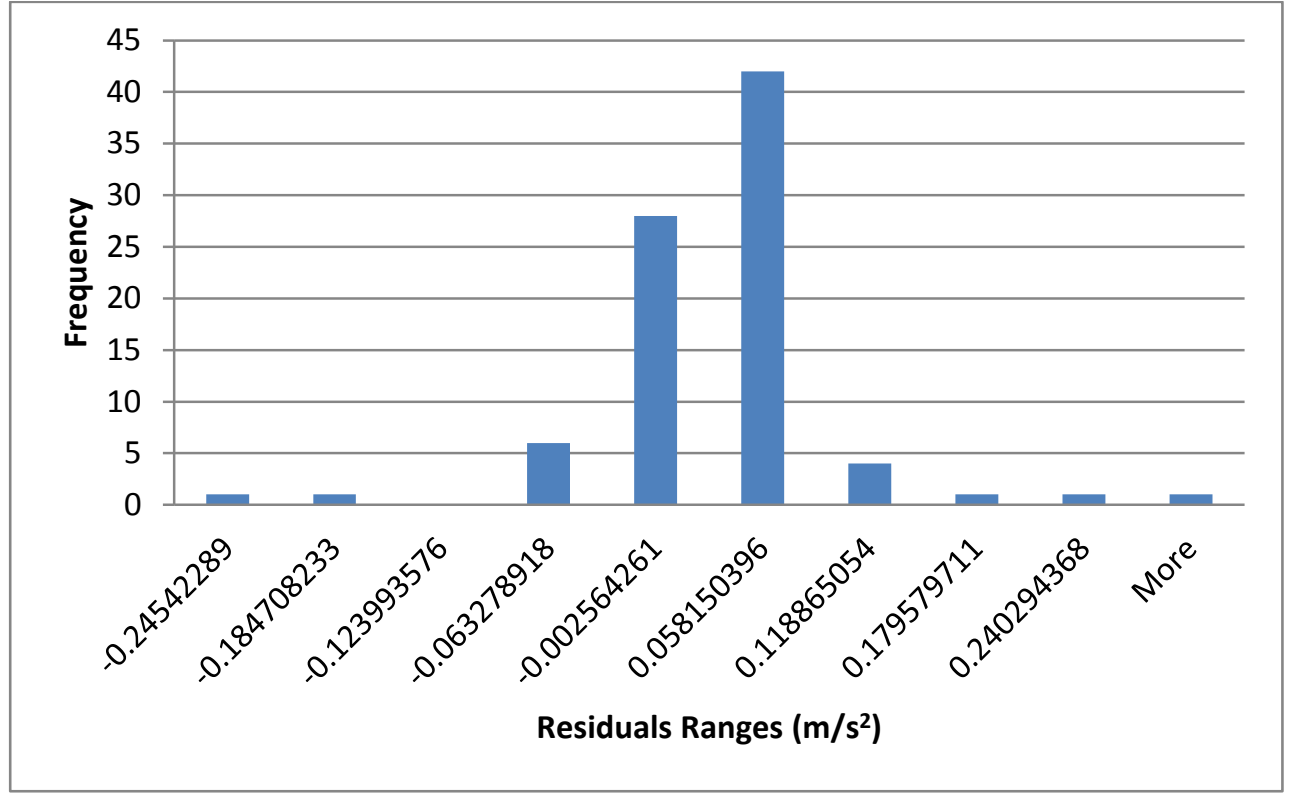

Figure 3: Residuals Histogram.

\section{Parkdale Ave. (W-NS)}

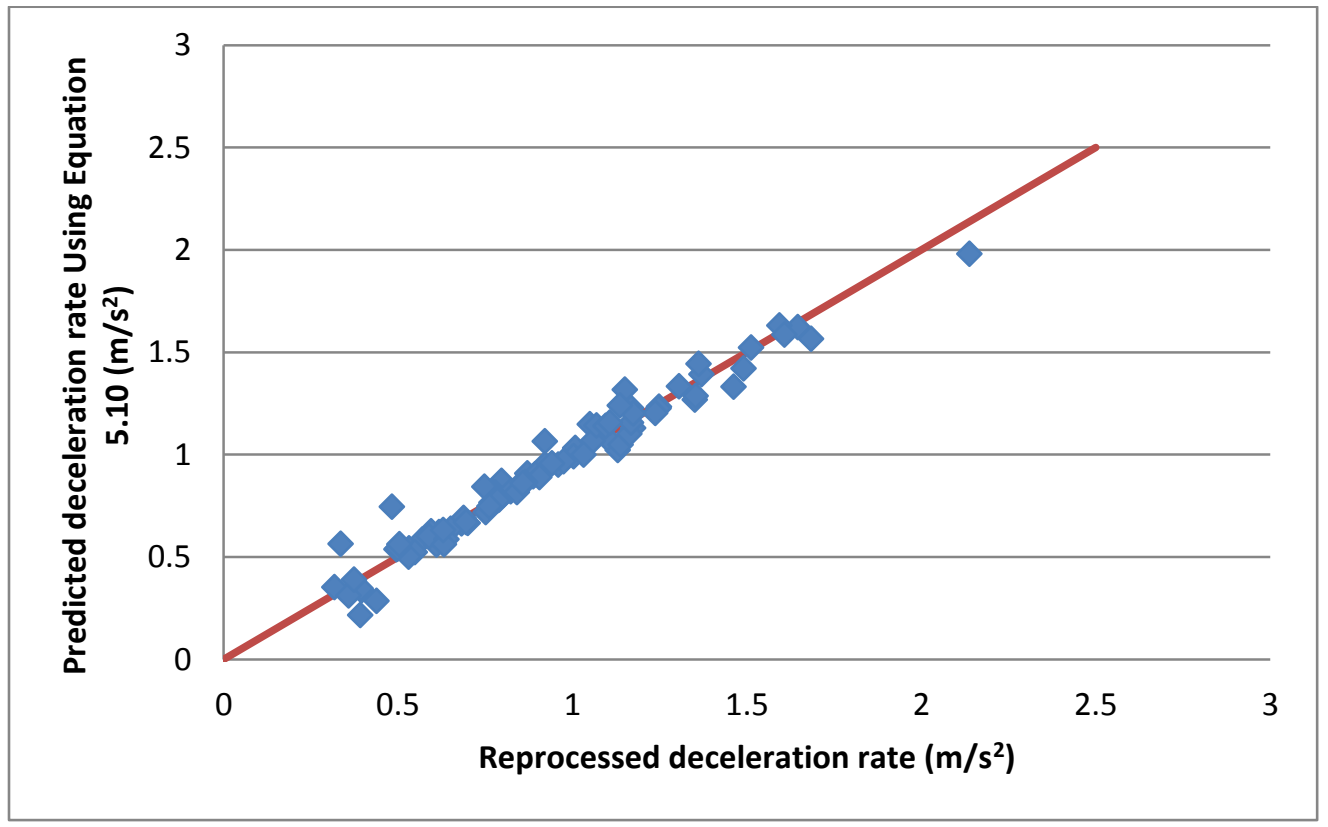

Figure 4: Line of Equality between Reprocessed Deceleration Rate and Predicted Deceleration Rate Using Equation 5.10. 


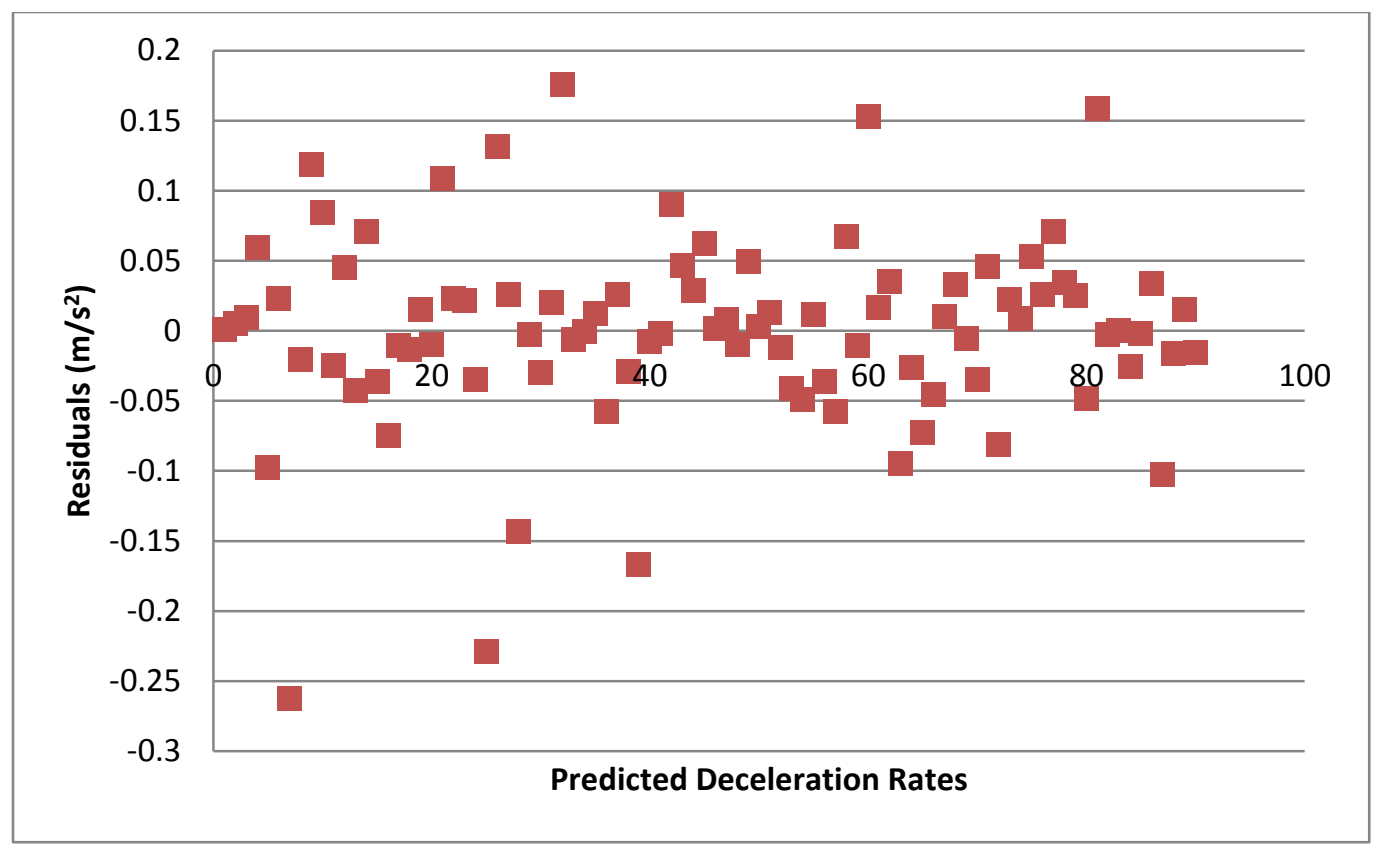

Figure 5: Residuals Plot for Each Predicted Deceleration Rate Using Equation 5.10.

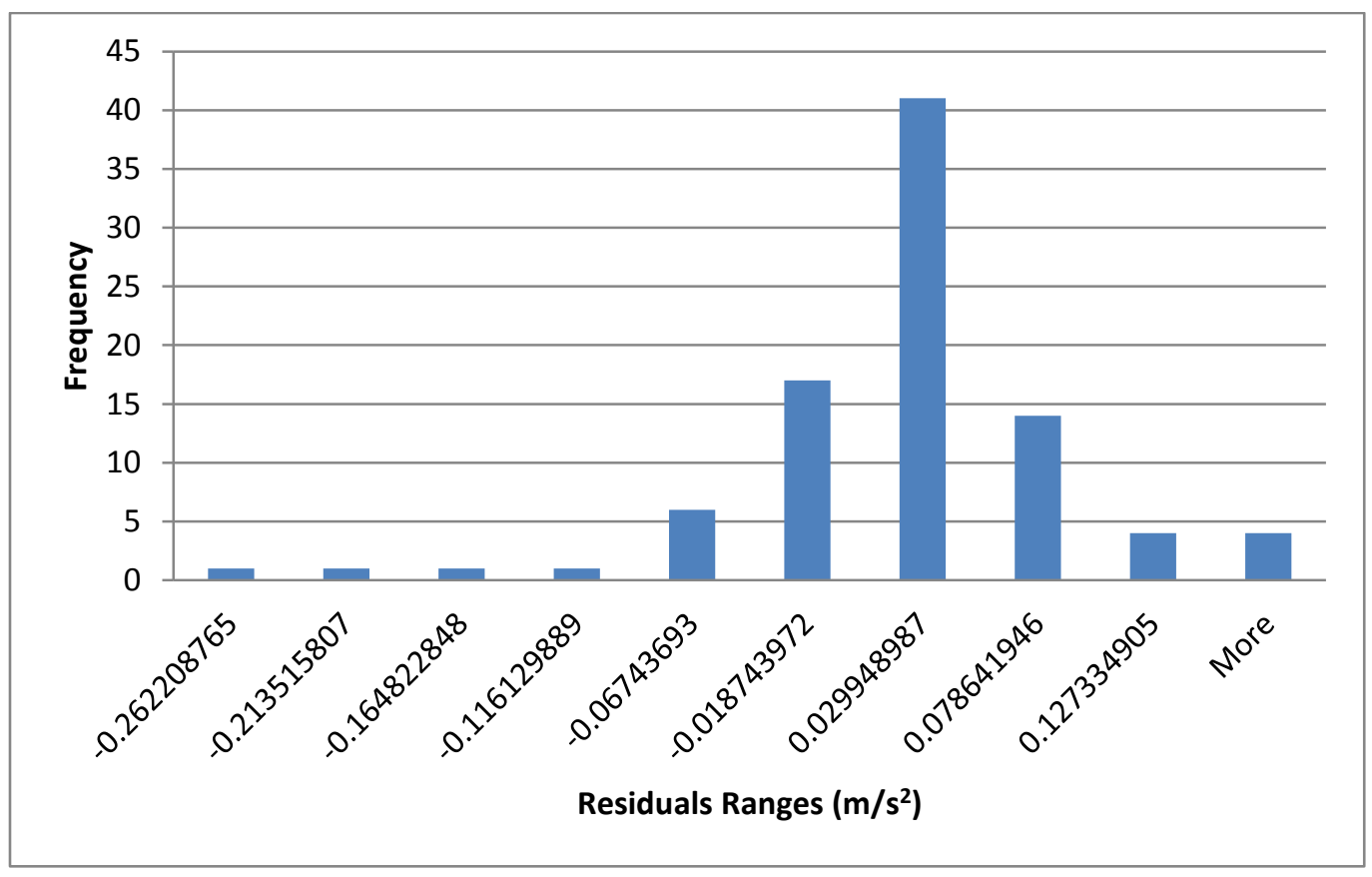

Figure 6: Residuals Histogram. 


\section{Bronson Ave. (W-NS)}

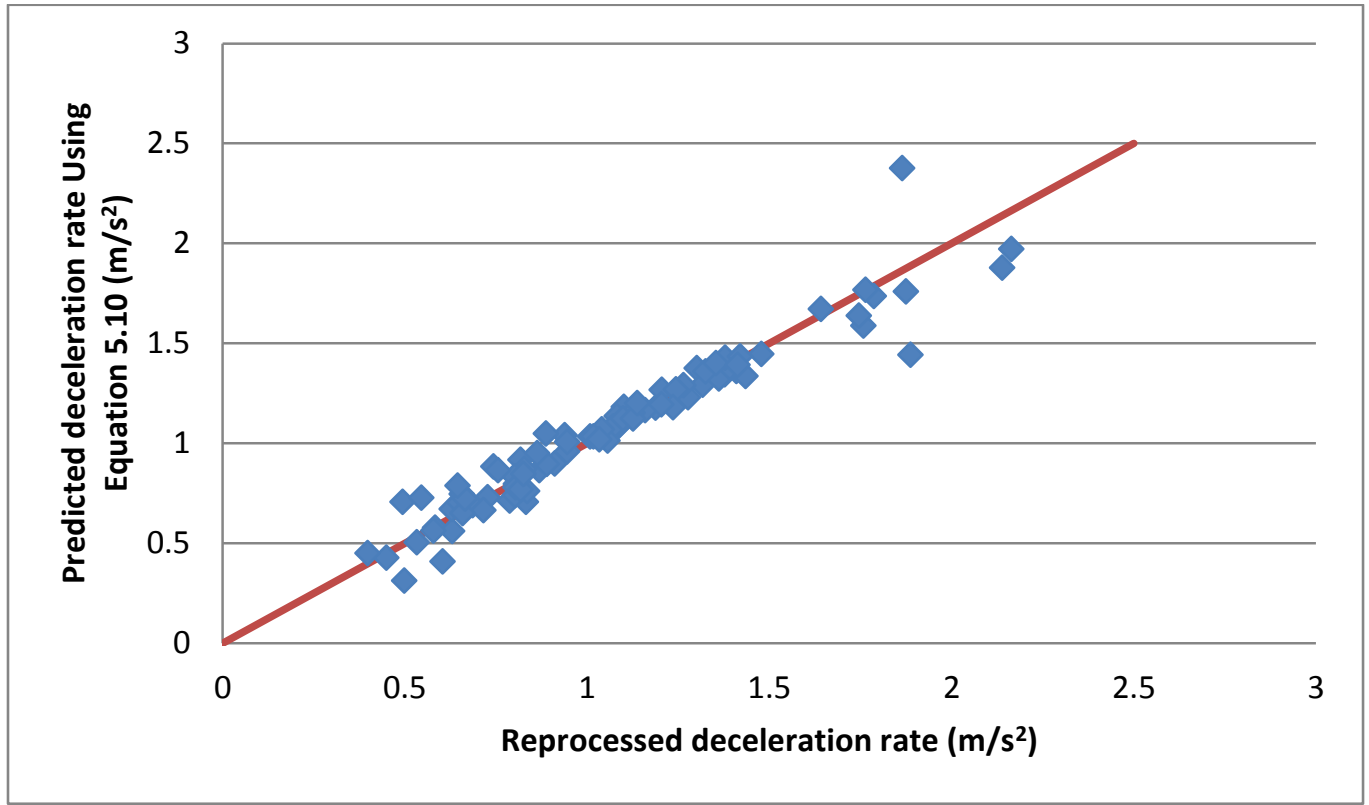

Figure 7: Line of Equality between Reprocessed Deceleration Rate and Predicted Deceleration Rate Using Equation 5.10.

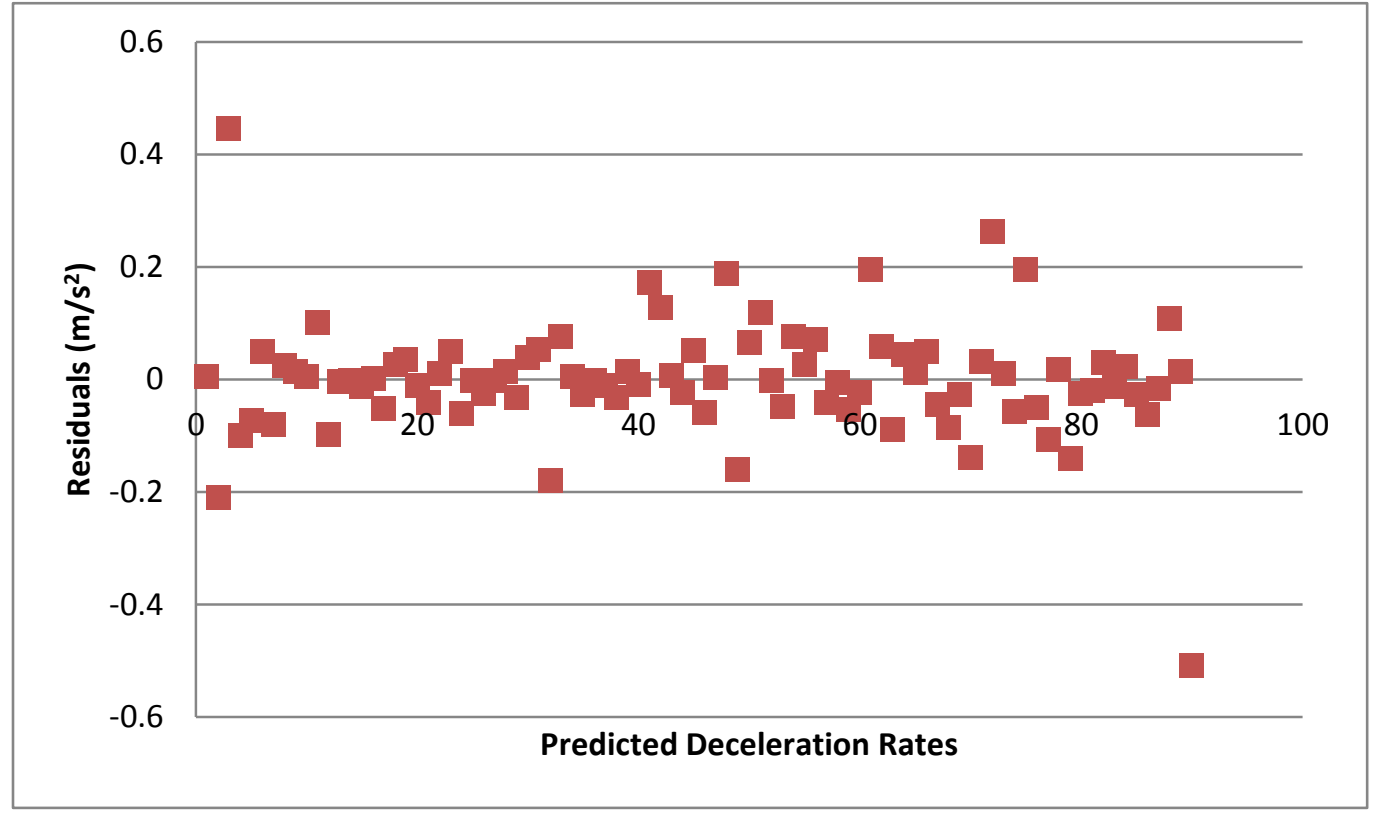

Figure 8: Residuals Plot for Each Each Predicted Deceleration Rate Using Equation

5.10 . 


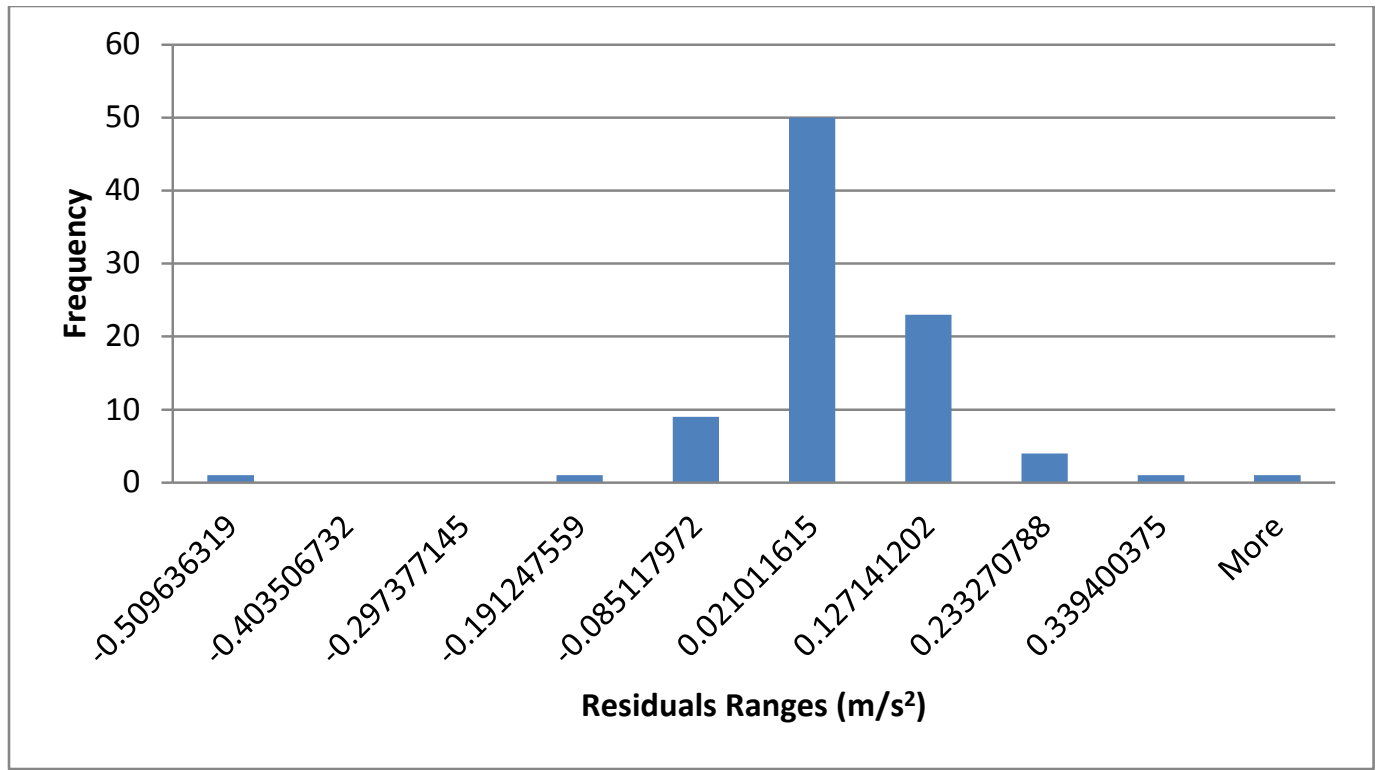

Figure 9: Residuals Histogram.

\section{Moodie Drive (W-NS)}

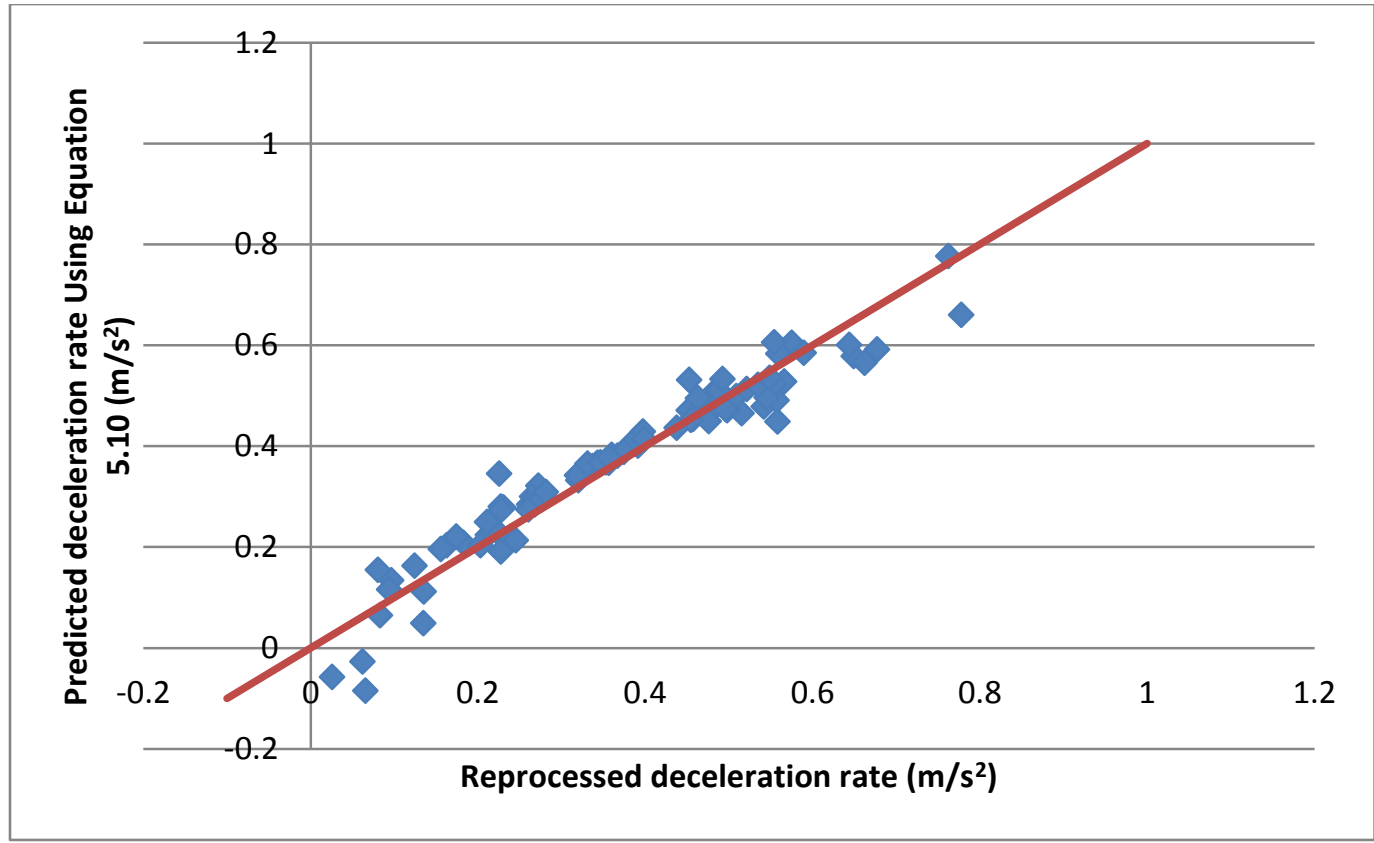

Figure 10: Line of Equality between Reprocessed Deceleration Rate and Predicted Deceleration Rate Using Equation 5.10. 


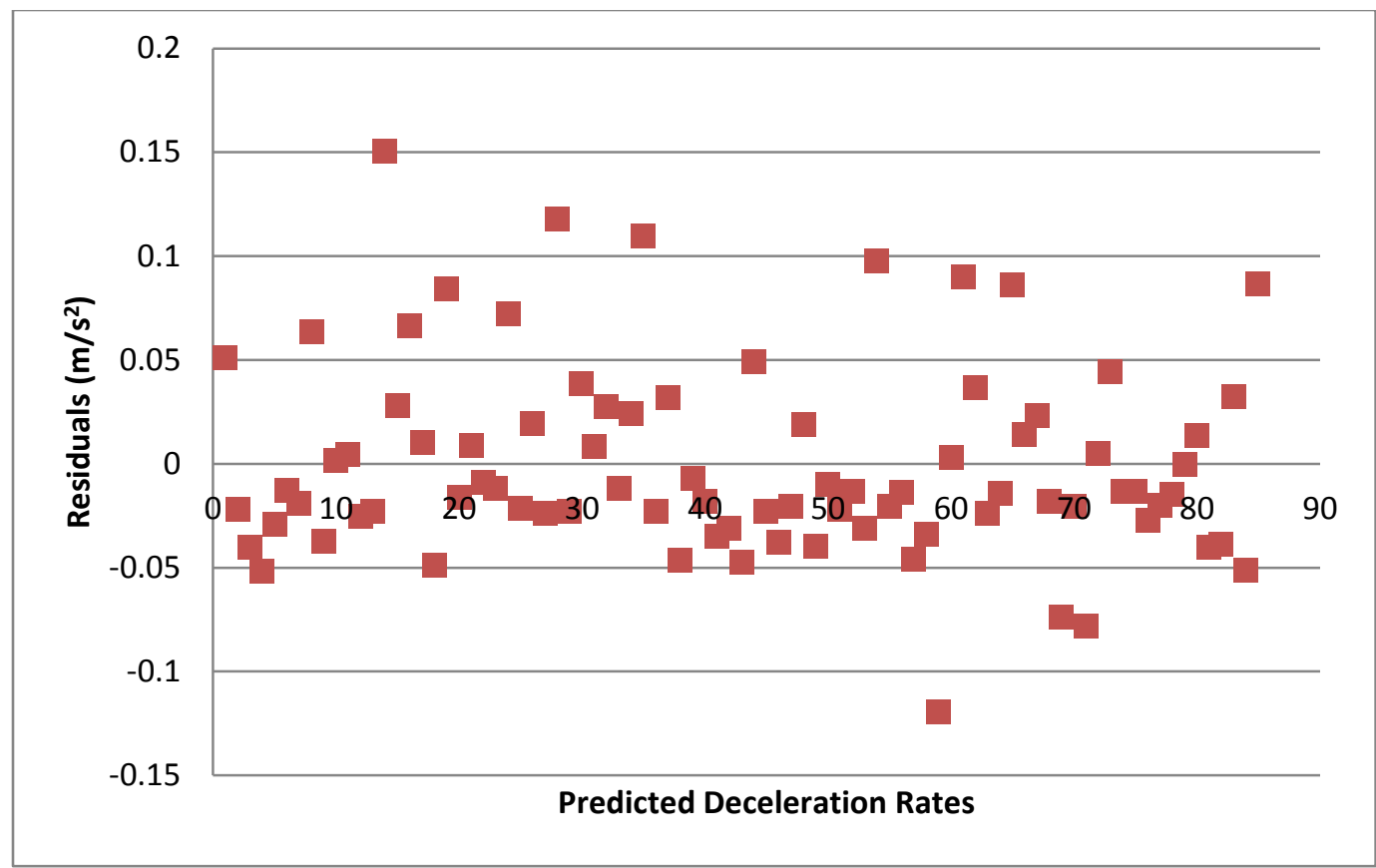

Figure 11: Residuals Plot for Each Predicted Deceleration Rate Using Equation 5.10.

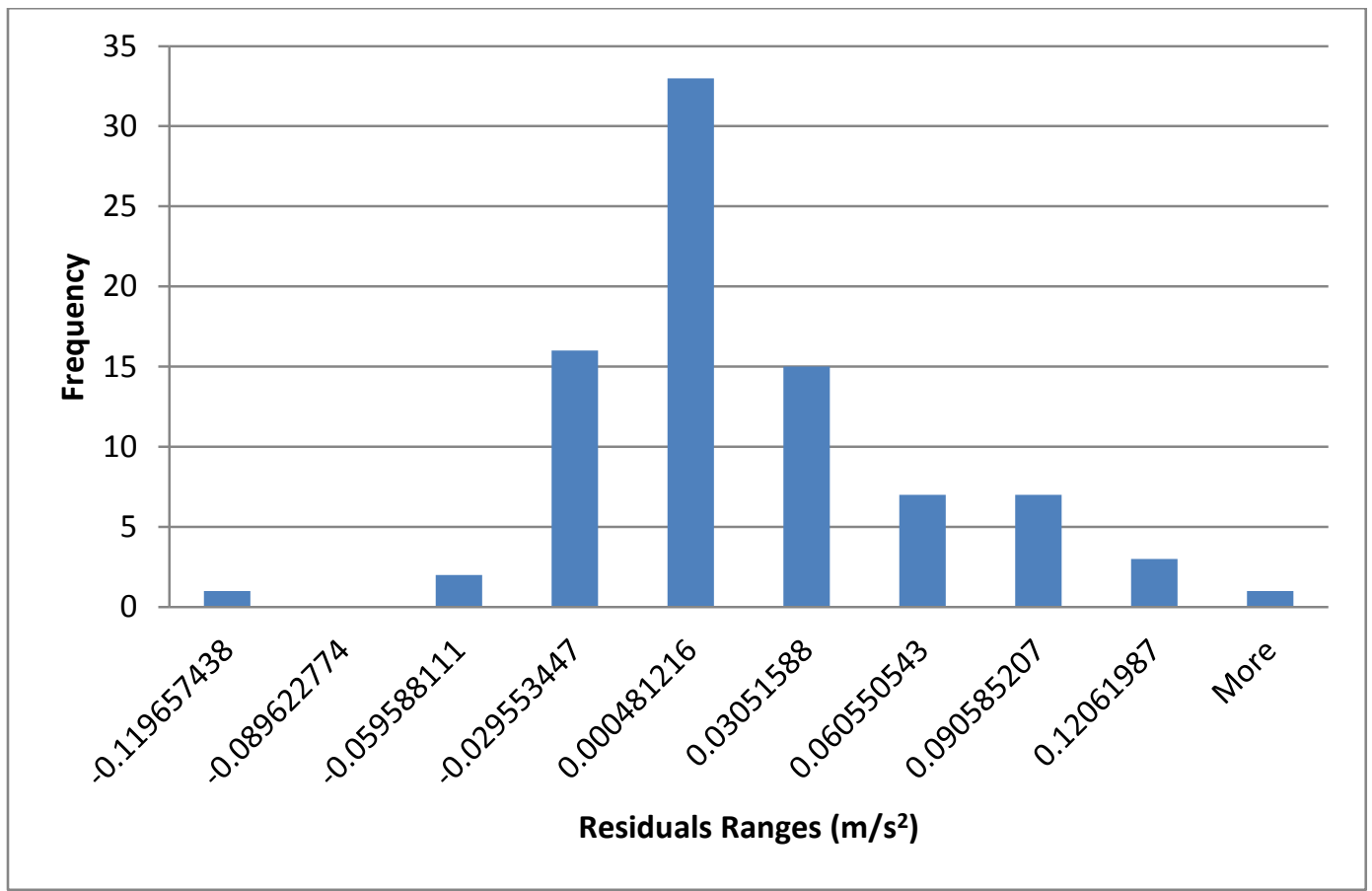

Figure 12: Residuals Histogram. 
Terry Fox Drive (E-NS)

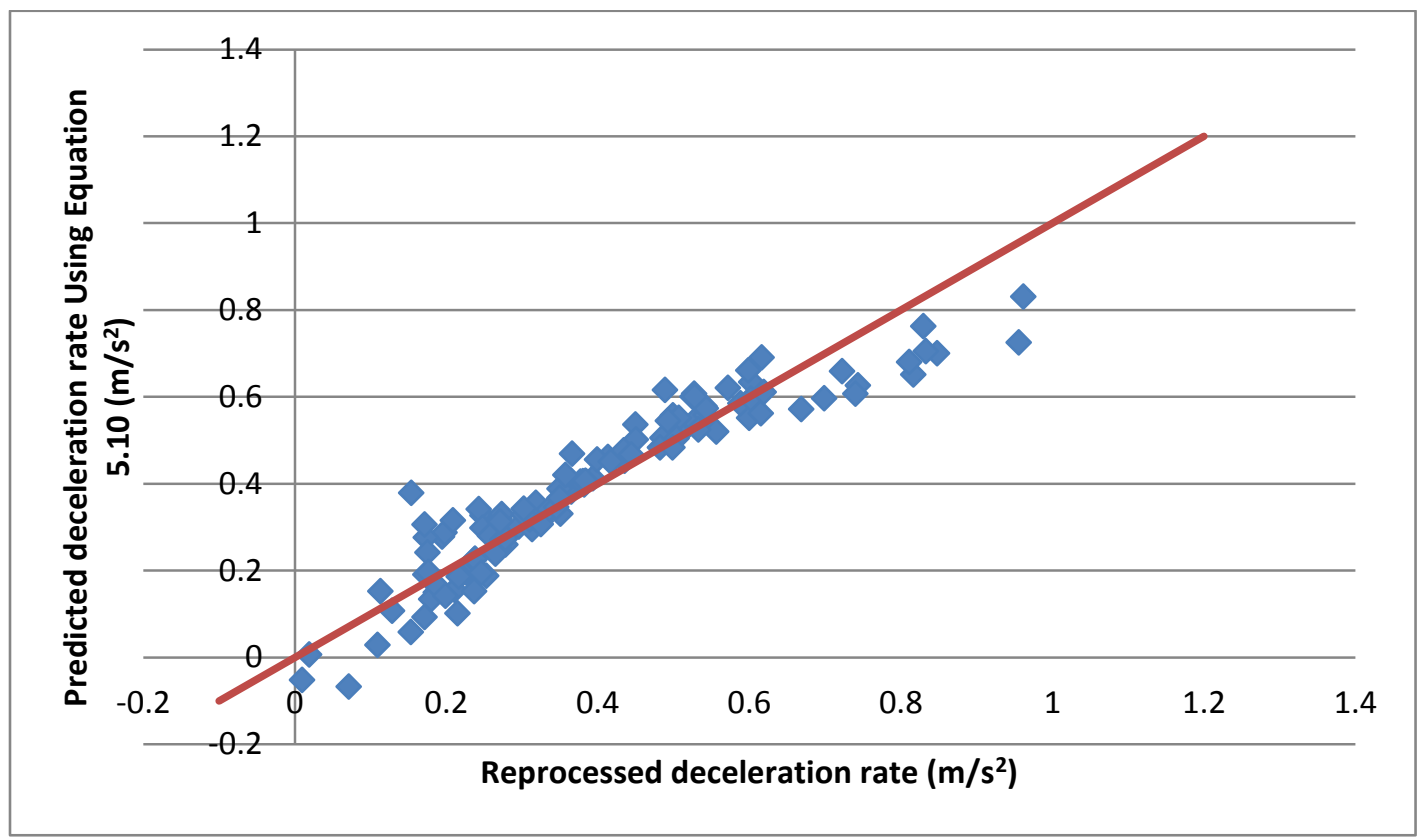

Figure 13: Line of Equality between Reprocessed Deceleration Rate and Predicted Deceleration Rate Using Equation 5.10.

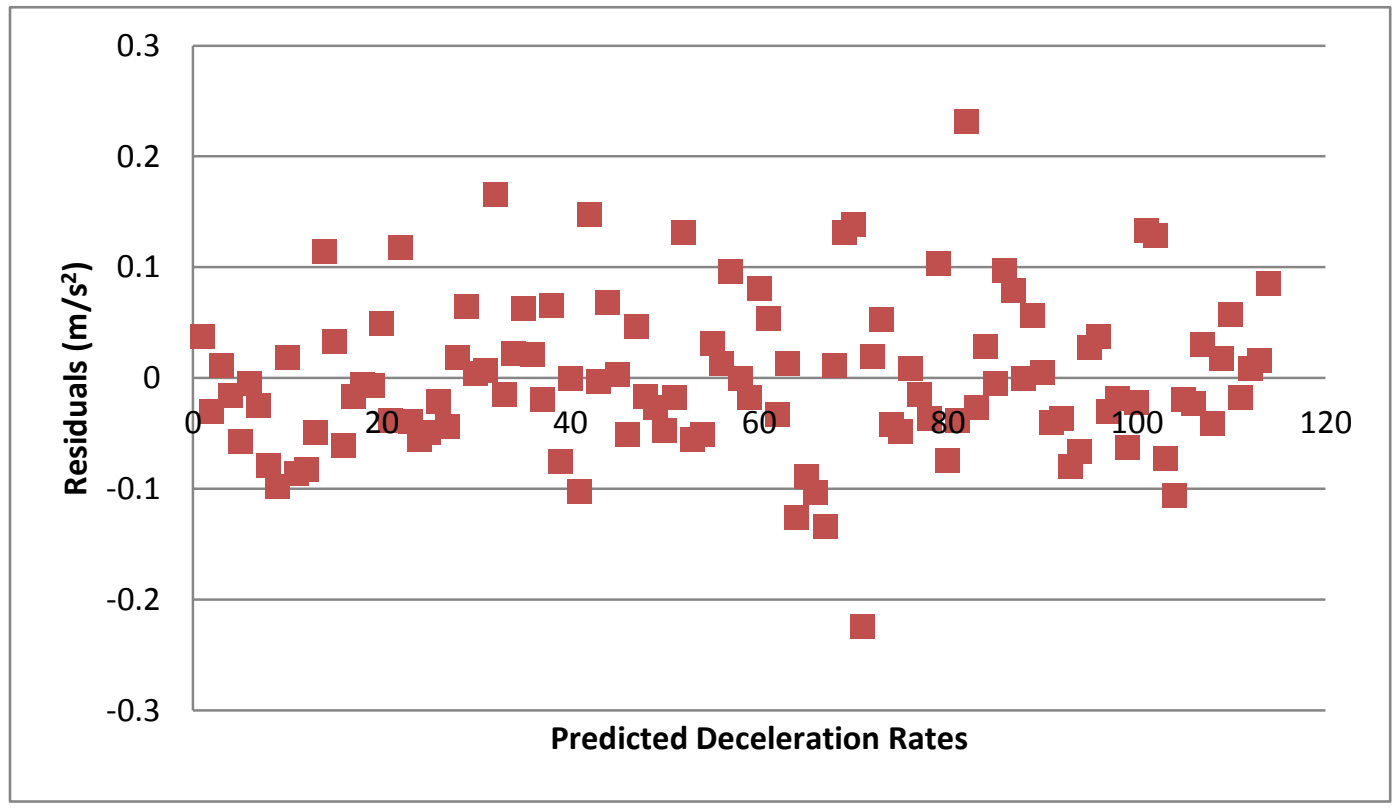

Figure 14: Residuals Plot for Each Predicted Deceleration Rate Using Equation 5.10. 


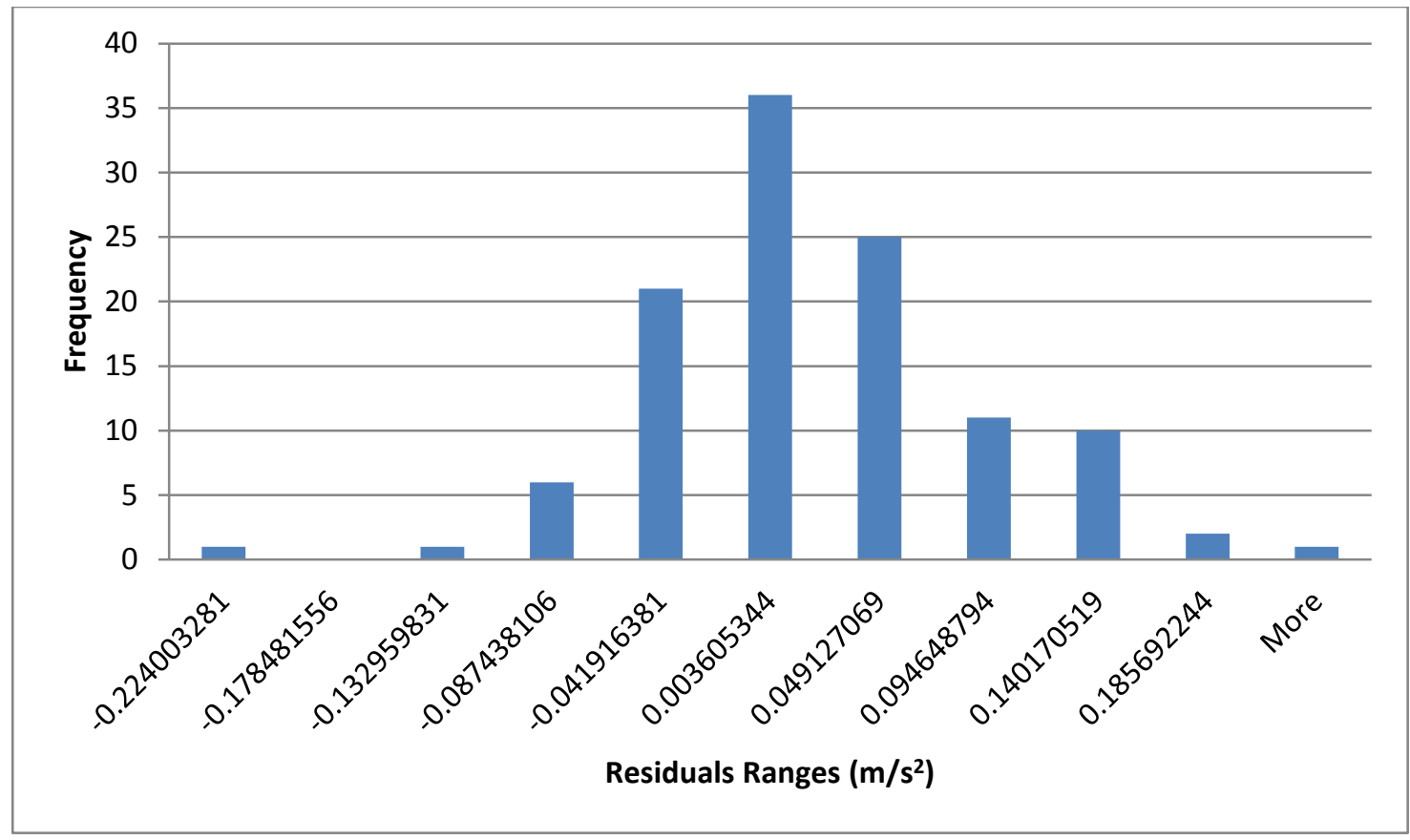

Figure 15: Residuals Histogram.

\section{Carp Road (E-NS)}

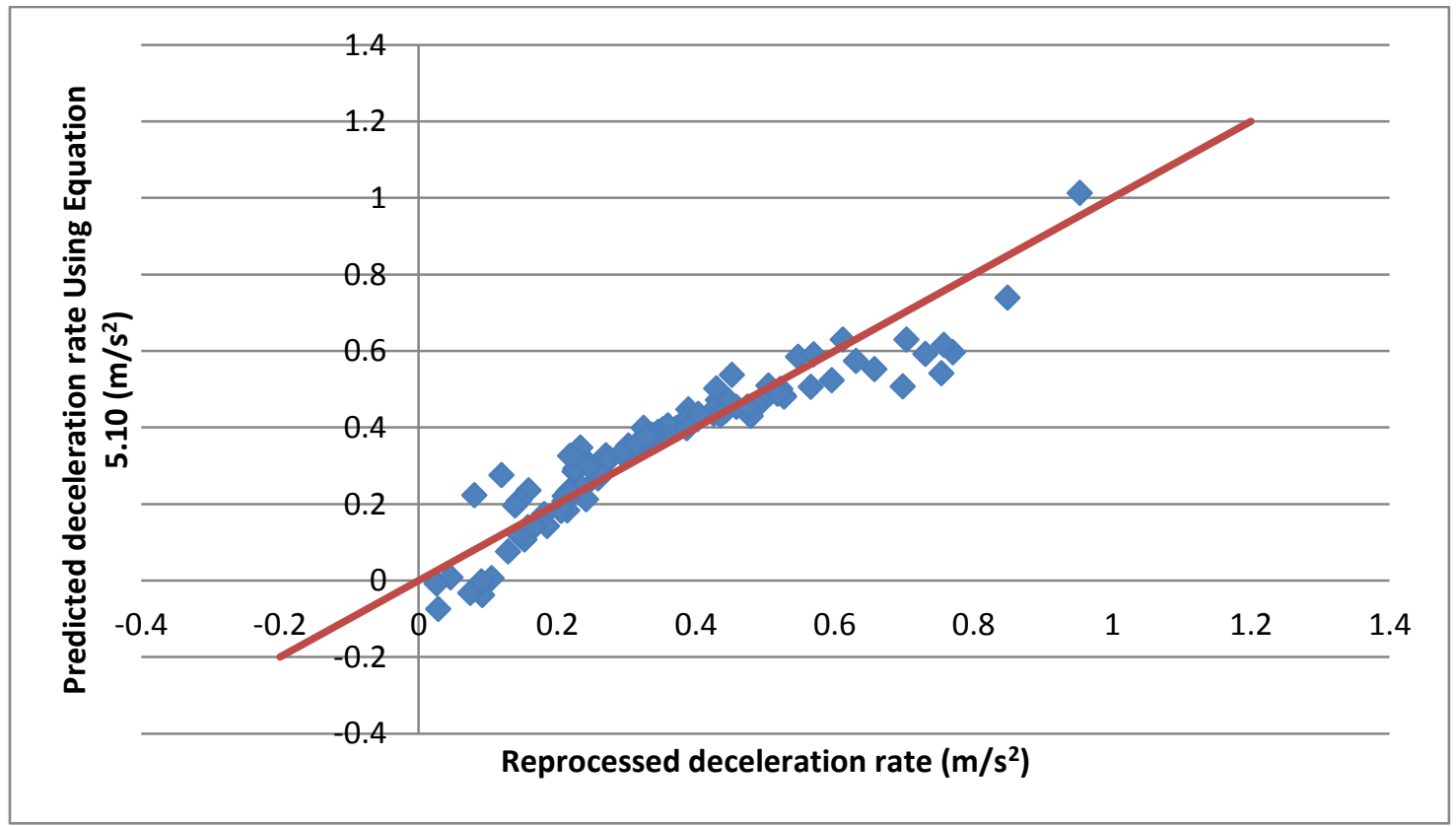

Figure 16: Line of Equality between Reprocessed Deceleration Rate and Predicted Deceleration Rate Using Equation 5.10. 


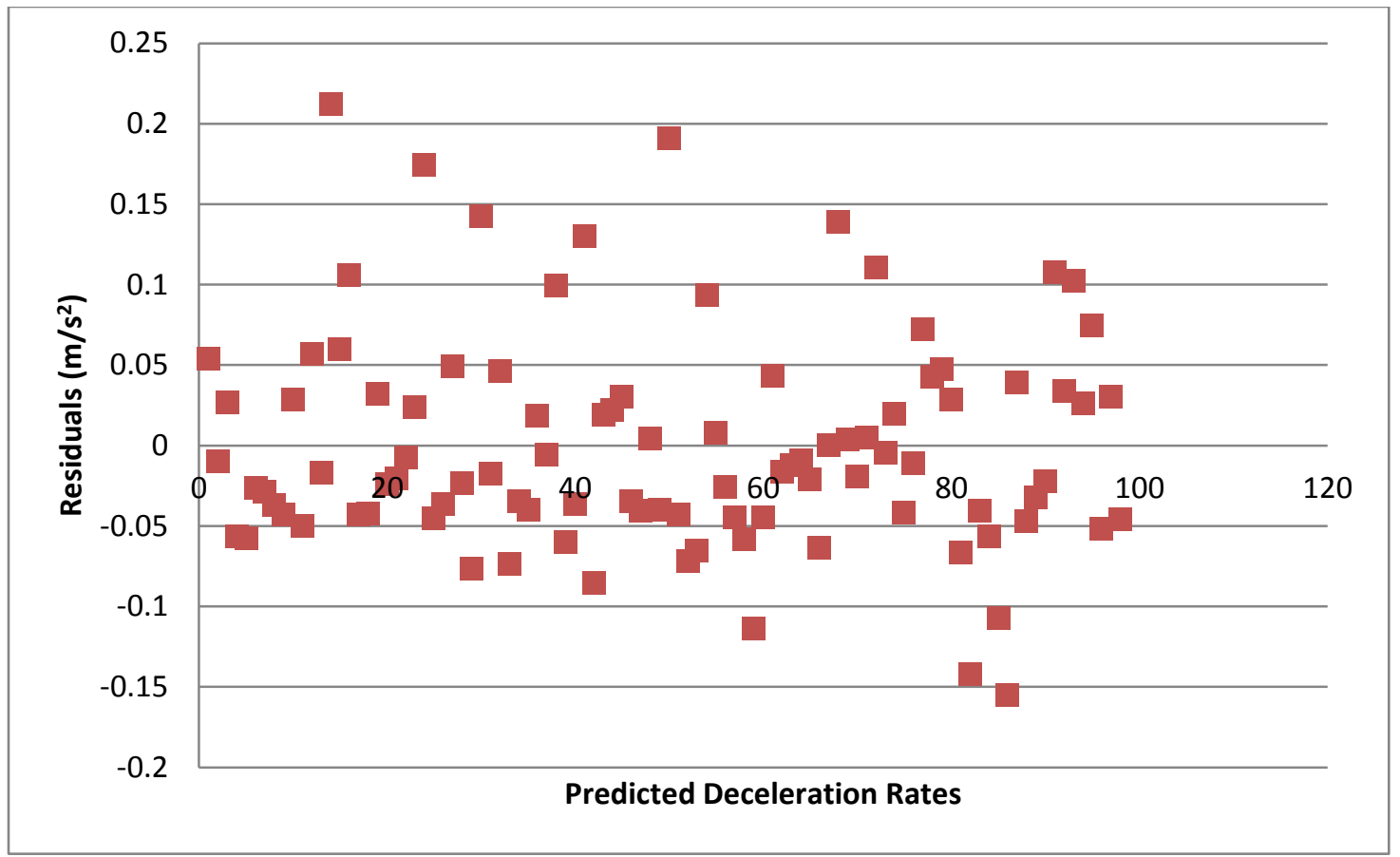

Figure 17: Residuals Plot for Each Predicted Deceleration Rate Using Equation 5.10.

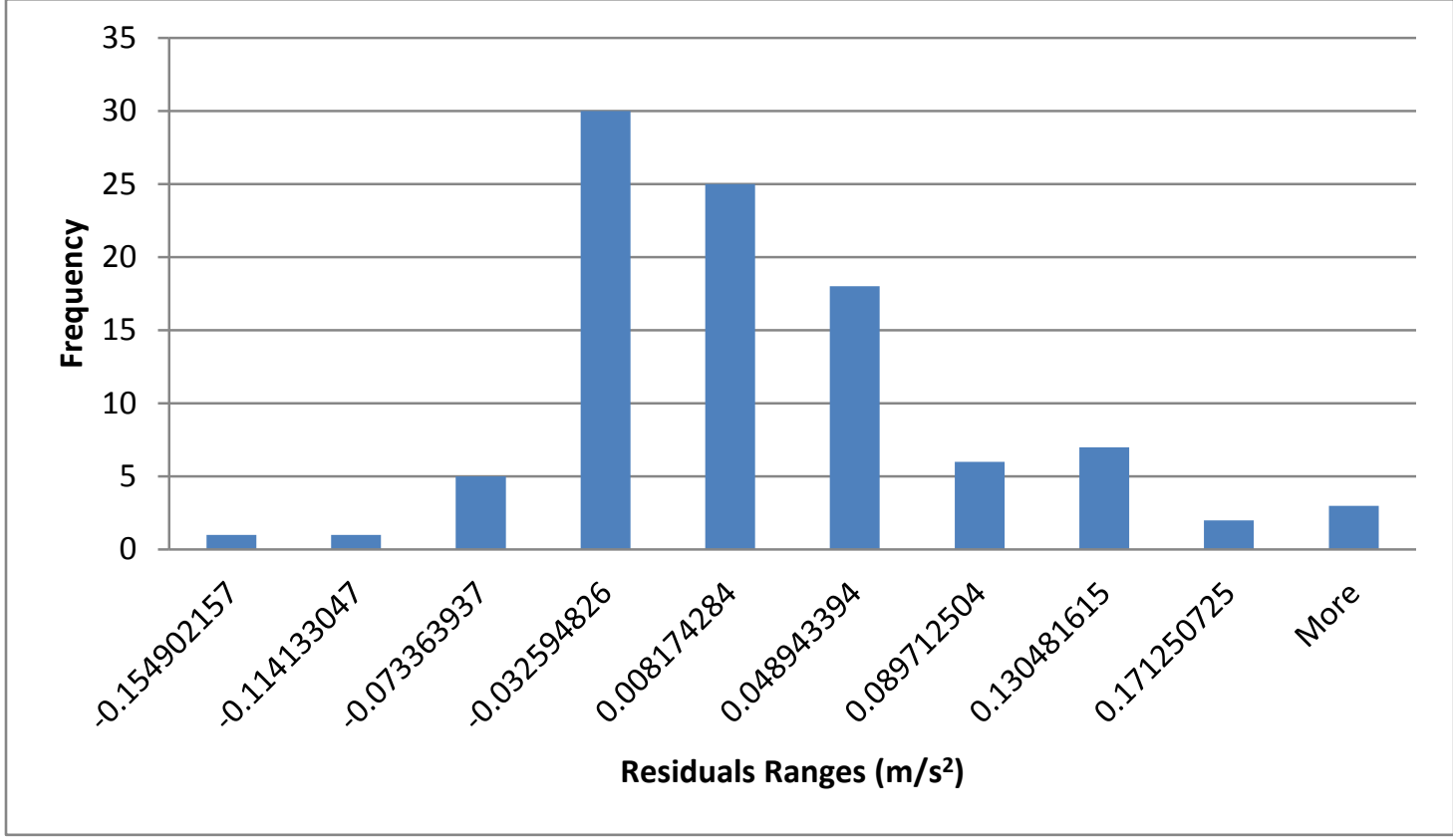

Figure 18: Residuals Histogram. 
Terry Fox Drive (W-NS)

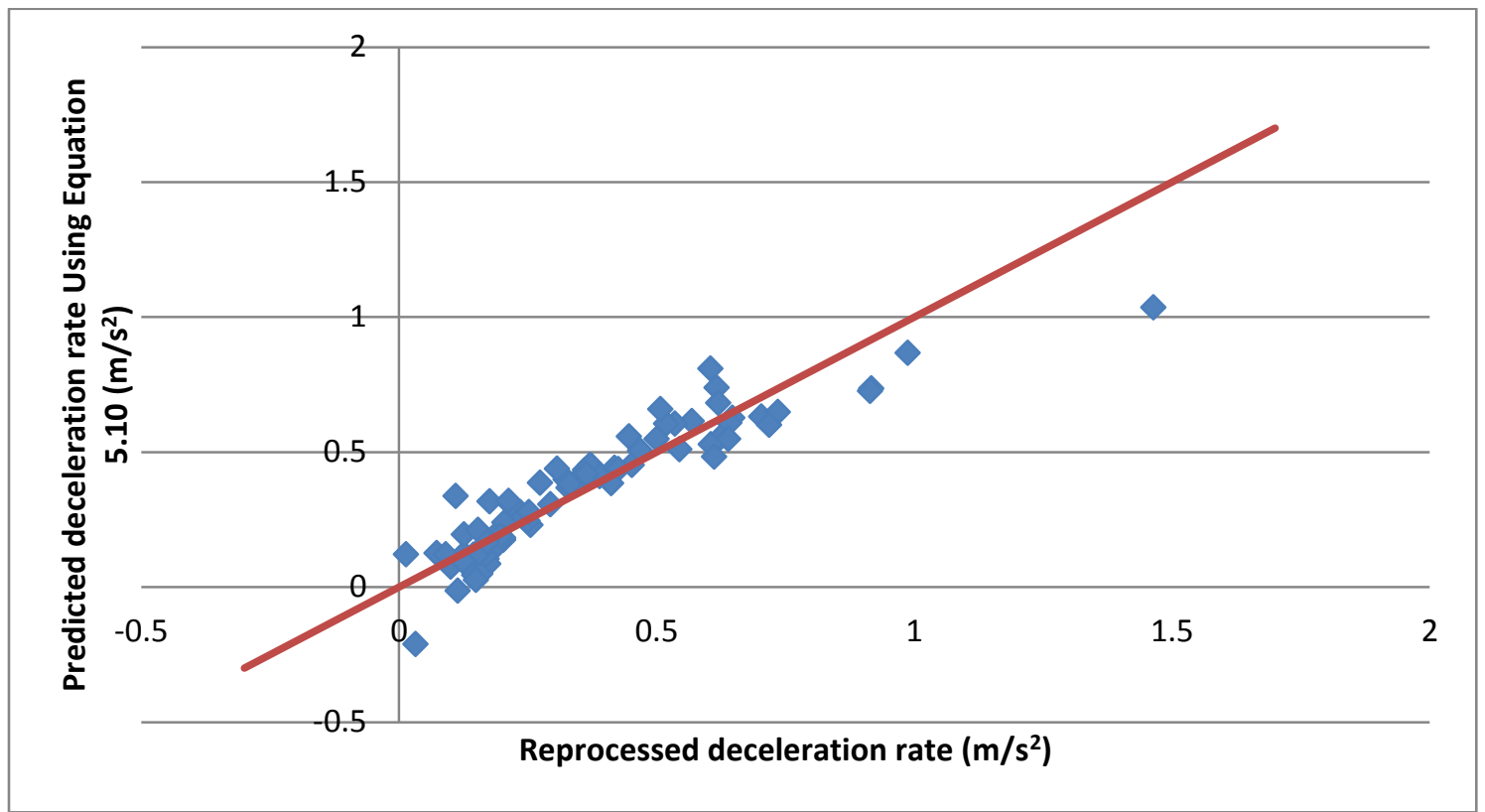

Figure 19: Line of Equality between Reprocessed Deceleration Rate and Predicted Deceleration Rate Using Equation 5.10.

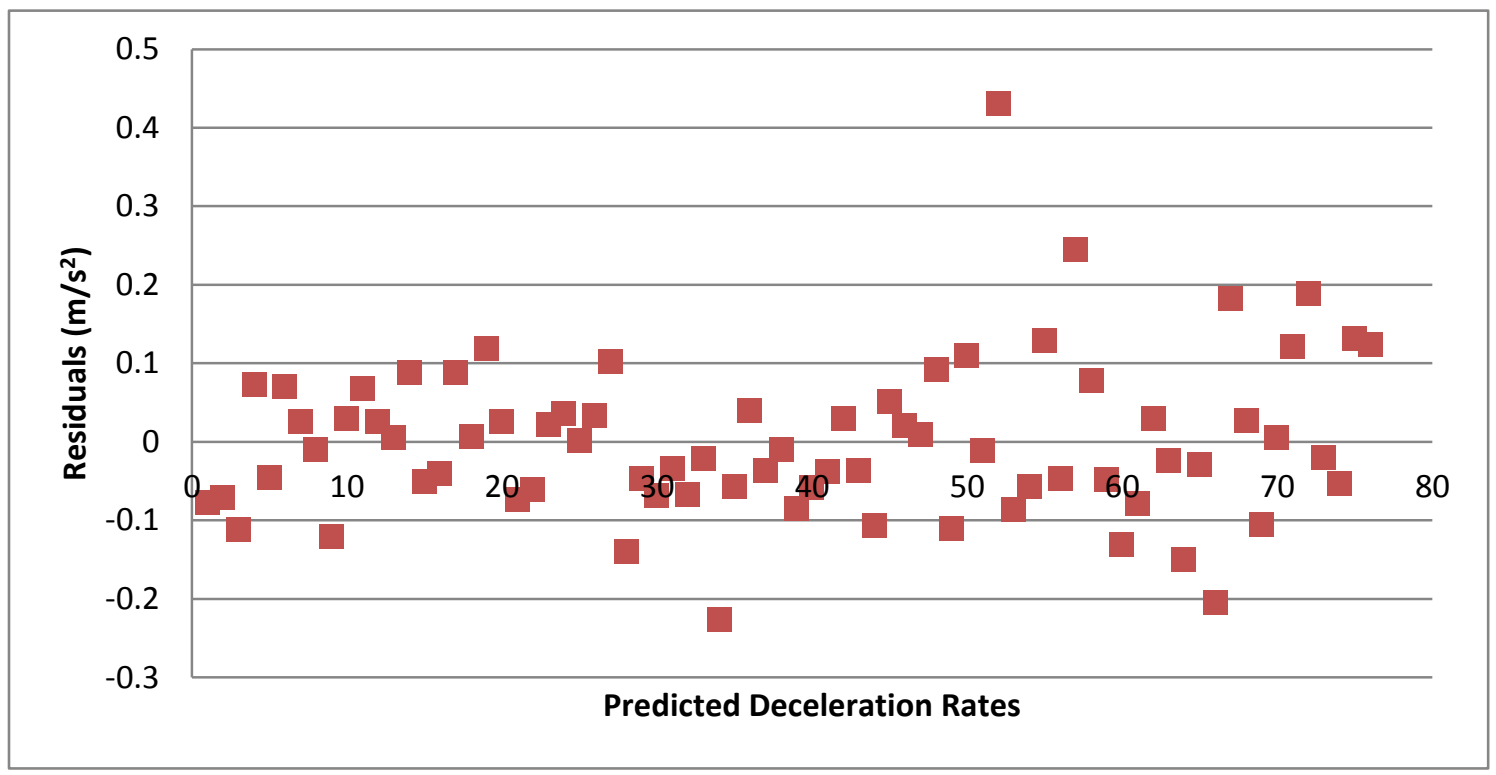

Figure 20: Residuals Plot for Each Predicted Deceleration Rate Using Equation 5.10. 


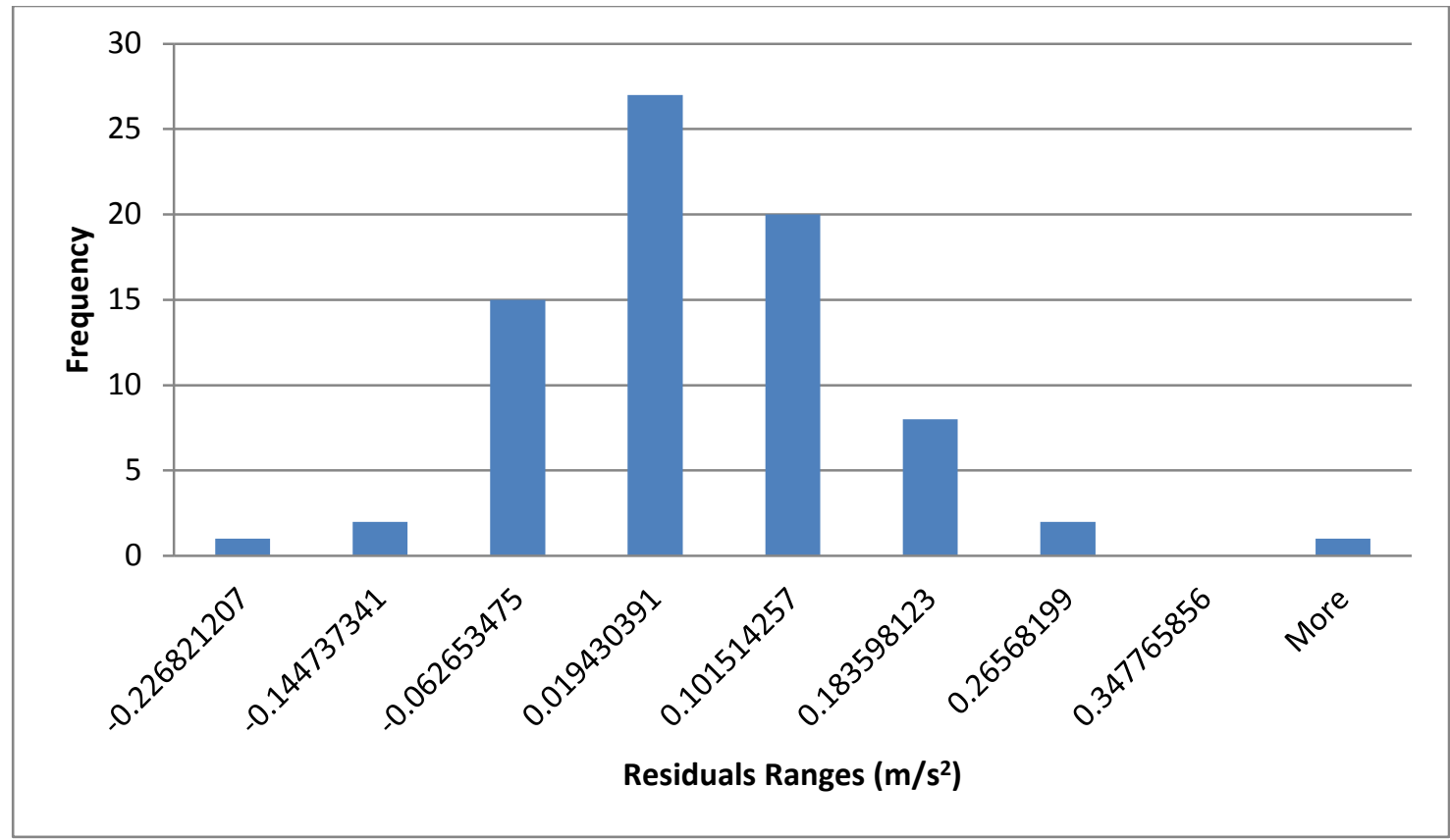

Figure 21: Residuals Histogram.

\section{All Sites Data Combined}

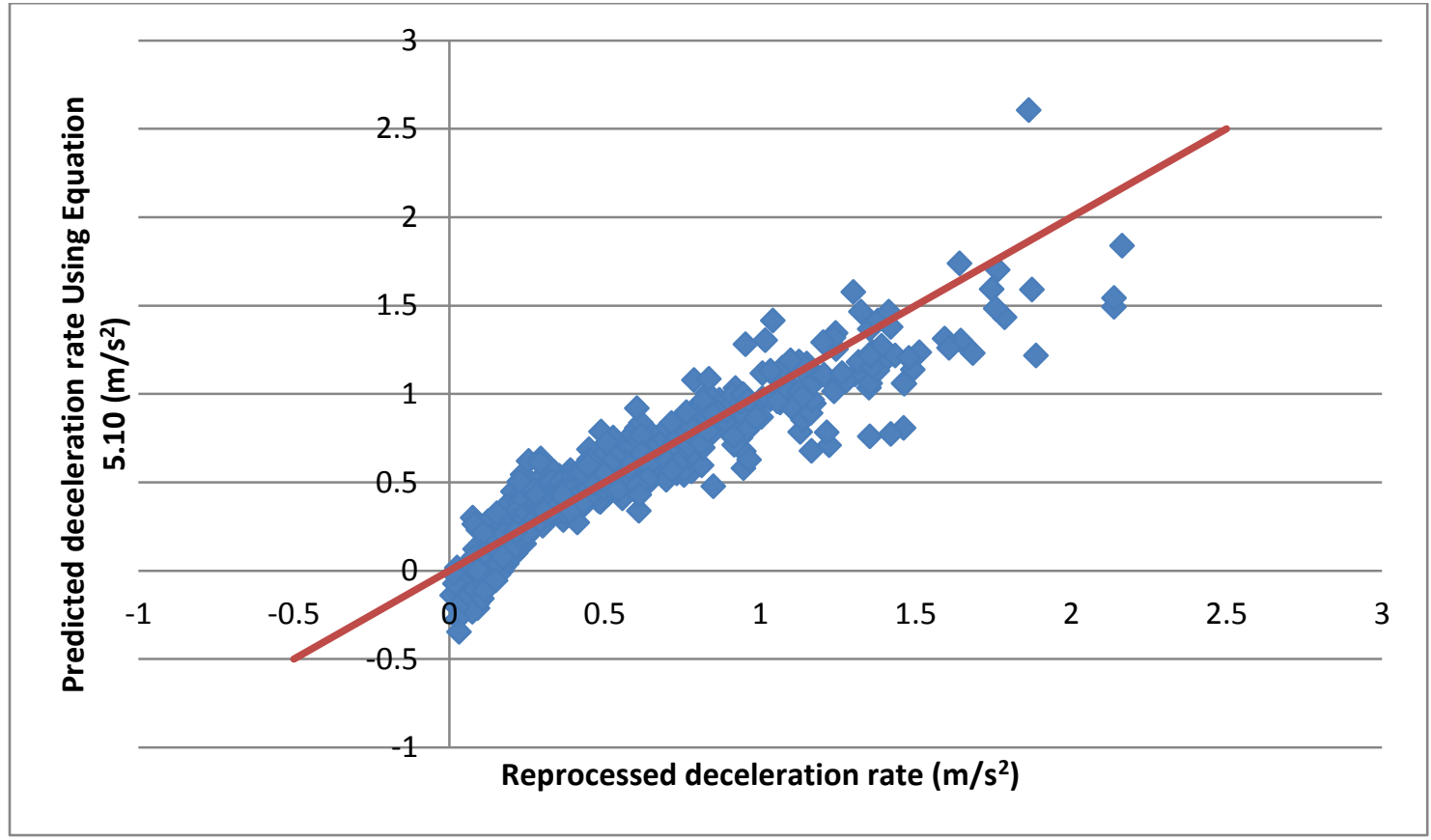

Figure 22: Line of Equality between Reprocessed Deceleration Rate and Predicted Deceleration Rate Using Equation 5.10. 


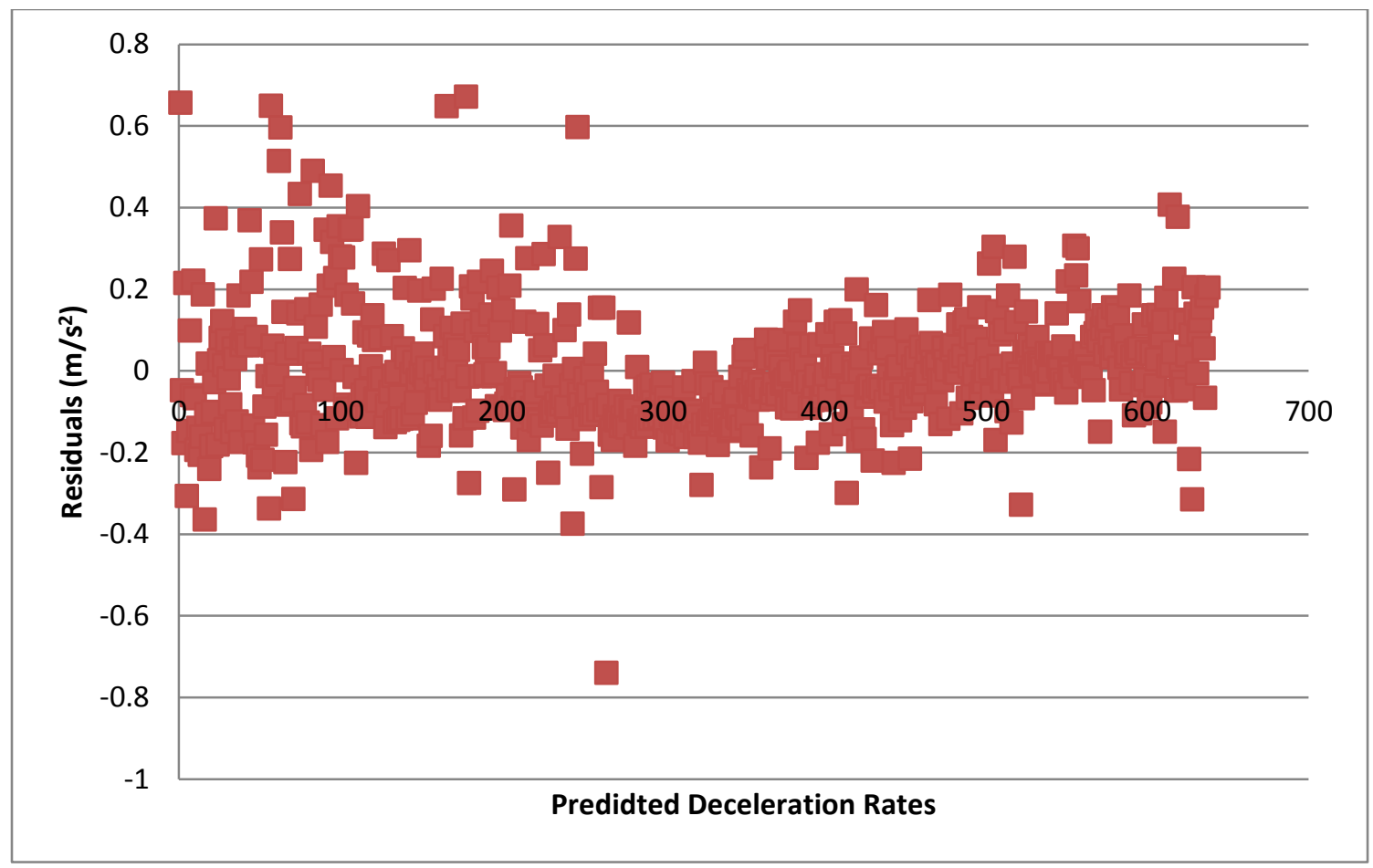

Figure 23: Residuals Plot for Each Predicted Deceleration Rate Using Equation 5.10.

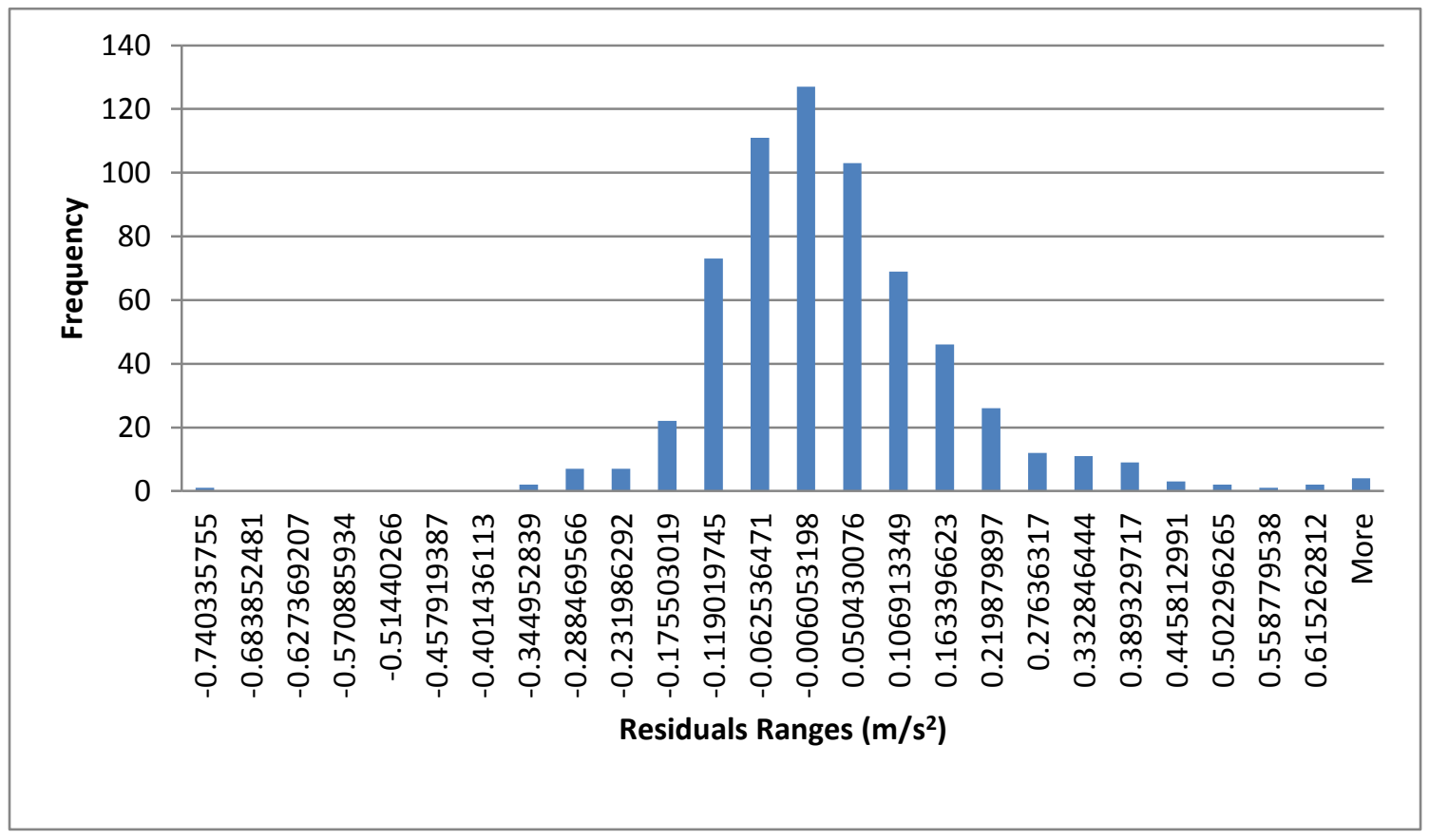

Figure 24: Residuals Histogram. 


\section{Appendix 8: Multiple Linear Regression between Mean of the Parameters and Standard Deviation.}


Table 26: Mean and Standard Deviations of Parameters at Each Site

\begin{tabular}{|c|c|c|c|c|c|c|c|c|c|}
\hline$v_{i}$ & $\begin{array}{c}\text { Available L } \\
(S L)\end{array}$ & $v_{f 85}$ & $U$ & $v_{f}$ & $d$ & $\operatorname{Std}\left(v_{i}\right)$ & $\operatorname{Std}\left(v_{f}\right)$ & $\operatorname{Std}(U)$ & $\operatorname{Std}(d)$ \\
\hline 20.576 & 58.000 & 21.410 & 0.570 & 19.663 & 0.540 & 2.162 & 2.214 & 0.153 & 0.286 \\
\hline 23.292 & 179.000 & 22.386 & 0.416 & 20.163 & 0.907 & 2.346 & 2.457 & 0.083 & 0.358 \\
\hline 24.188 & 212.000 & 21.872 & 0.474 & 19.251 & 1.070 & 2.251 & 2.724 & 0.094 & 0.393 \\
\hline 28.384 & 292.000 & 29.542 & 0.474 & 26.621 & 0.369 & 2.575 & 2.564 & 0.134 & 0.175 \\
\hline 28.661 & 388.000 & 28.330 & 0.517 & 25.979 & 0.392 & 2.391 & 2.582 & 0.177 & 0.203 \\
\hline 29.257 & 430.000 & 28.985 & 0.503 & 26.843 & 0.358 & 2.421 & 2.249 & 0.220 & 0.193 \\
\hline 29.499 & 446.000 & 29.775 & 0.474 & 27.119 & 0.371 & 2.875 & 2.773 & 0.205 & 0.256 \\
\hline
\end{tabular}

Table 27: Modeling Mean $v_{i}$

\begin{tabular}{|c|c|c|c|c|c|c|c|c|}
\hline & & & & & & & \\
\hline \multicolumn{2}{|c|}{$\begin{array}{l}\text { Regression Statistics } \\
\text { Multinle } \mathrm{R}\end{array}$} & & & & & & & \\
\hline R Square & 0.980 & & & & & & & \\
\hline \multicolumn{9}{|l|}{ Adjusted } \\
\hline R Square & 0.970 & & & & & & & \\
\hline \multicolumn{9}{|l|}{ Standard } \\
\hline Error & 0.613 & & & & & & & \\
\hline \multicolumn{9}{|l|}{ Observati } \\
\hline ons & 7.000 & & & & & & & \\
\hline \multicolumn{9}{|l|}{ ANOVA } \\
\hline & & \multicolumn{7}{|c|}{ Significance } \\
\hline & $d f$ & SS & $M S$ & $F$ & $F$ & & & \\
\hline \multicolumn{9}{|l|}{ Regressio } \\
\hline $\mathrm{n}$ & 2.000 & 73.651 & 36.826 & 98.143 & 0.000 & & & \\
\hline Residual & 4.000 & 1.501 & 0.375 & & & & & \\
\hline \multirow[t]{3}{*}{ Total } & 6.000 & 75.152 & & & & & & \\
\hline & & Standard & & $p-$ & Lower & Upper & Lower & Upper \\
\hline & Coefficients & Error & $t$ Stat & value & $95 \%$ & $95 \%$ & $95.0 \%$ & $95.0 \%$ \\
\hline $\begin{array}{l}\text { Intercept } \\
\text { Available }\end{array}$ & 11.584 & 2.731 & 4.241 & 0.013 & 4.000 & 19.167 & 4.000 & 19.167 \\
\hline L & 0.014 & 0.004 & 3.615 & 0.022 & 0.003 & 0.024 & 0.003 & 0.024 \\
\hline Vf85 & 0.413 & 0.140 & 2.953 & 0.042 & 0.025 & 0.801 & 0.025 & 0.801 \\
\hline
\end{tabular}


Table 28: Modeling Mean $\boldsymbol{v}_{f}$

\begin{tabular}{lr}
\hline \multicolumn{2}{c}{ Regression Statistics } \\
\hline Multiple R & 0.999 \\
R Square & 0.997 \\
Adjusted & \\
R Square & 0.996 \\
$\begin{array}{l}\text { Standard } \\
\text { Error } \\
\text { Observati } \\
\text { ons }\end{array}$ & 0.235 \\
\hline
\end{tabular}

ANOVA

\begin{tabular}{|c|c|c|c|c|c|c|c|c|}
\hline & \multirow[b]{2}{*}{$d f$} & \multirow[b]{2}{*}{ SS } & \multirow[b]{2}{*}{$M S$} & \multirow{2}{*}{\multicolumn{2}{|c|}{$\begin{array}{c}\text { Significance } \\
F\end{array}$}} & & & \\
\hline & & & & & & & & \\
\hline \multicolumn{9}{|l|}{ Regressio } \\
\hline $\mathrm{n}$ & 2.000 & 83.662 & 41.831 & 755.930 & 0.000 & & & \\
\hline Residual & 4.000 & 0.221 & 0.055 & & & & & \\
\hline \multirow[t]{3}{*}{ Total } & 6.000 & 83.883 & & & & & & \\
\hline & & Standard & & $p-$ & Lower & Upper & Lower & Upper \\
\hline & Coefficients & Error & t Stat & value & $95 \%$ & $95 \%$ & $95.0 \%$ & $95.0 \%$ \\
\hline Intercept & 1.762 & 1.179 & 1.495 & 0.209 & -1.511 & 5.036 & -1.511 & 5.036 \\
\hline Vf85 & 0.967 & 0.025 & 38.306 & 0.000 & 0.897 & 1.037 & 0.897 & 1.037 \\
\hline StdVf & -1.310 & 0.457 & -2.864 & 0.046 & -2.580 & -0.040 & -2.580 & -0.040 \\
\hline
\end{tabular}

- Mean U was found to have no relation with any of the parameters 


\section{Table 29: Modeling Std $\left(v_{\mathrm{i}}\right)$ Using Mean $\boldsymbol{v}_{\boldsymbol{f}}$}

\begin{tabular}{|c|c|c|c|c|c|c|c|c|}
\hline \multicolumn{2}{|c|}{ Regression Statistics } & & & & & & & \\
\hline Multiple R & 0.759 & & & & & & & \\
\hline R Square & 0.576 & & & & & & & \\
\hline \multicolumn{9}{|l|}{ Adjusted R } \\
\hline Square & 0.492 & & & & & & & \\
\hline \multicolumn{9}{|l|}{ Standard } \\
\hline Error & 0.167 & & & & & & & \\
\hline \multicolumn{9}{|l|}{ Observation } \\
\hline$s$ & 7.000 & & & & & & & \\
\hline \multicolumn{9}{|l|}{ ANOVA } \\
\hline & & \multicolumn{7}{|c|}{ Significance } \\
\hline & $d f$ & SS & $M S$ & $F$ & $F$ & & & \\
\hline Regression & 1.000 & 0.191 & 0.191 & 6.805 & 0.048 & & & \\
\hline Residual & 5.000 & 0.140 & 0.028 & & & & & \\
\hline \multirow[t]{2}{*}{ Total } & 6.000 & 0.331 & & & & & & \\
\hline & $\begin{array}{c}\text { Coefficient } \\
s \\
\end{array}$ & $\begin{array}{l}\text { Standar } \\
d \text { Error }\end{array}$ & $t$ Stat & $p$-value & $\begin{array}{c}\text { Lower } \\
95 \%\end{array}$ & $\begin{array}{c}\text { Upper } \\
95 \%\end{array}$ & $\begin{array}{l}\text { Lower } \\
95.0 \% \\
\end{array}$ & $\begin{array}{l}\text { Upper } \\
95.0 \% \\
\end{array}$ \\
\hline Intercept & 1.303 & 0.437 & 2.979 & 0.031 & 0.178 & 2.427 & 0.178 & 2.427 \\
\hline $\mathrm{Vf}$ & 0.048 & 0.018 & 2.609 & 0.048 & 0.001 & 0.095 & 0.001 & 0.095 \\
\hline
\end{tabular}


Table 30: Modeling Std (vi) Using Mean v85thf

\begin{tabular}{|c|c|c|c|c|c|c|c|c|}
\hline \multicolumn{2}{|c|}{ Regression Statistics } & & & & & & & \\
\hline Multiple R & 0.786 & & & & & & & \\
\hline R Square & 0.618 & & & & & & & \\
\hline \multicolumn{9}{|l|}{ Adjusted R } \\
\hline Square & 0.541 & & & & & & & \\
\hline \multicolumn{9}{|l|}{ Standard } \\
\hline Error & 0.159 & & & & & & & \\
\hline Observations & 7.000 & & & & & & & \\
\hline \multicolumn{9}{|l|}{ ANOVA } \\
\hline & & \multicolumn{7}{|c|}{ Significance } \\
\hline & $d f$ & SS & $M S$ & $F$ & $F$ & & & \\
\hline Regression & 1.000 & 0.205 & 0.205 & 8.085 & 0.036 & & & \\
\hline Residual & 5.000 & 0.127 & 0.025 & & & & & \\
\hline \multirow[t]{2}{*}{ Total } & 6.000 & 0.331 & & & & & & \\
\hline & $\begin{array}{c}\text { Coefficie } \\
n t s\end{array}$ & $\begin{array}{l}\text { Standar } \\
d \text { Error }\end{array}$ & t Stat & P-value & $\begin{array}{c}\text { Lower } \\
95 \%\end{array}$ & $\begin{array}{c}\text { Upper } \\
95 \%\end{array}$ & $\begin{array}{l}\text { Lower } \\
95.0 \%\end{array}$ & $\begin{array}{l}\text { Upper } \\
95.0 \%\end{array}$ \\
\hline Intercept & 1.205 & 0.435 & 2.768 & 0.039 & 0.086 & 2.325 & 0.086 & 2.325 \\
\hline Vf85 & 0.047 & 0.017 & 2.843 & 0.036 & 0.005 & 0.090 & 0.005 & 0.090 \\
\hline
\end{tabular}

- $\quad \operatorname{Std}\left(v_{f}\right)$ was found to have no relation with any of the parameters

- $\quad \operatorname{Std}(U)$ was found to have no relation with any of the parameters 
Appendix 9: MCS Script. 


\section{Using Each Site's Data}

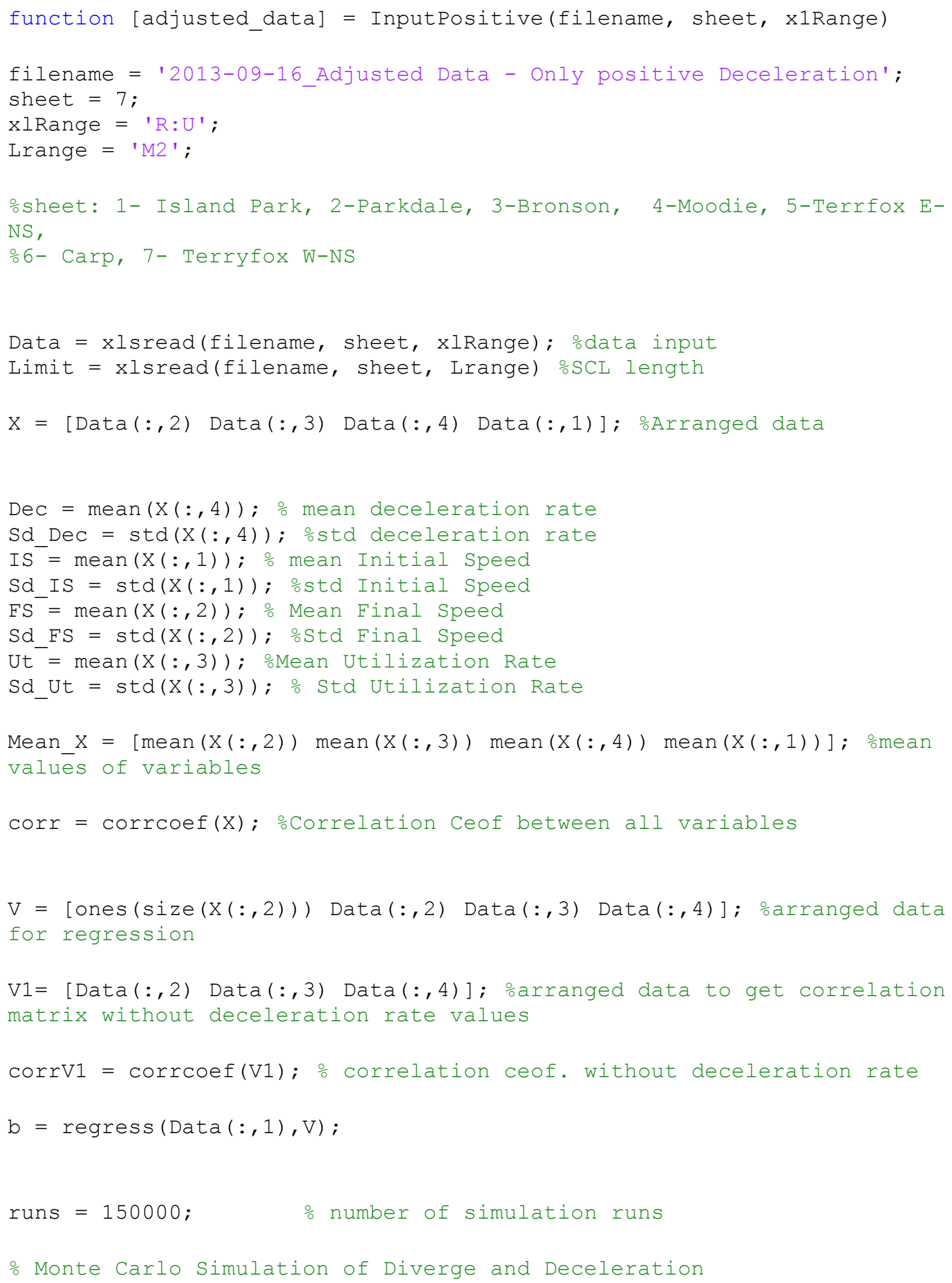




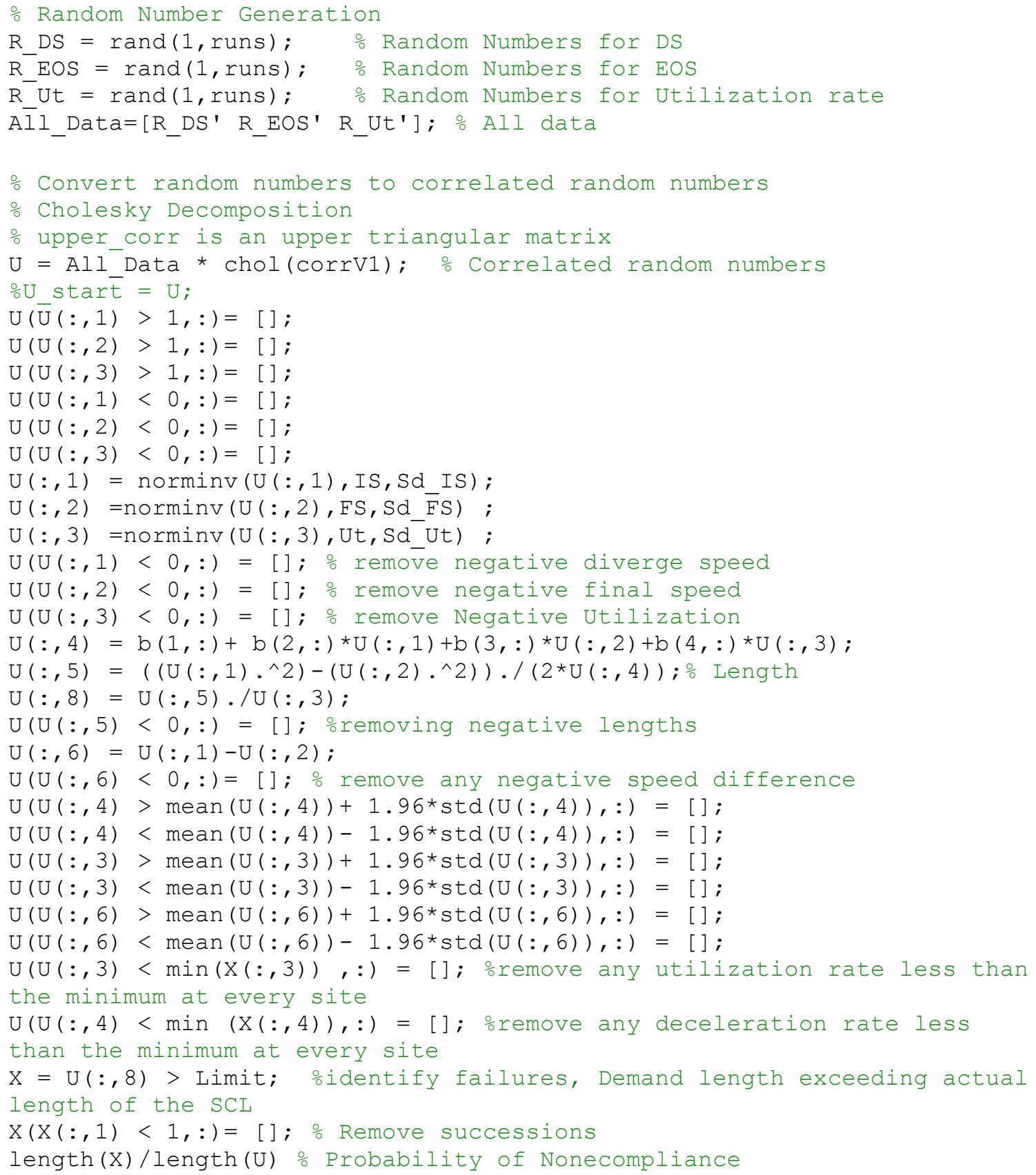

end 


\section{Using All Sites Combined Data}

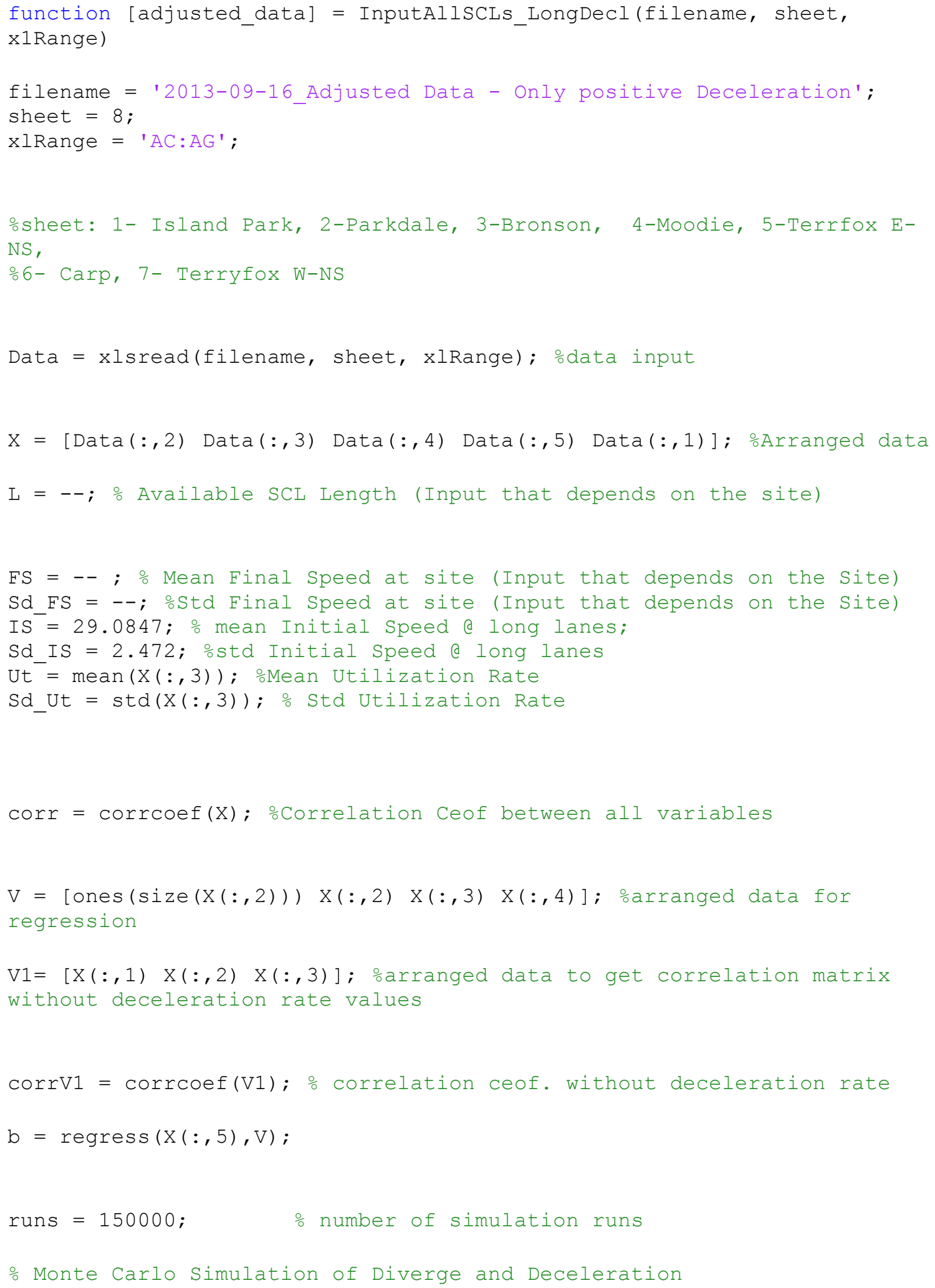




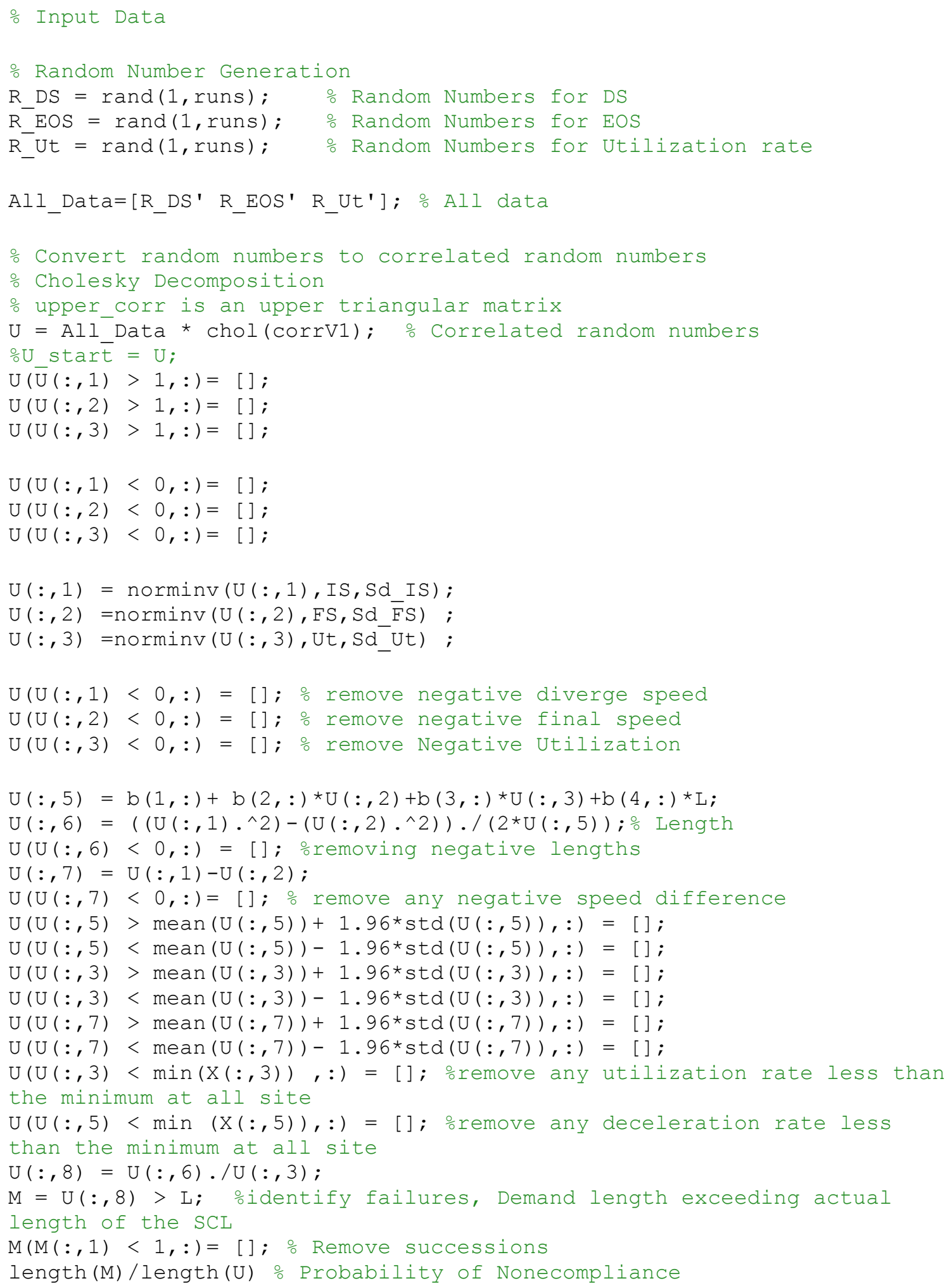


Appendix 10: FORM Script. 


\section{Using Each Site's Data}

function $\mathrm{b}=\operatorname{ell}\left(\mathrm{x}, \mathrm{MS}, \mathrm{C}_{-}\right.$inv)

$\mathrm{A}=\mathrm{x}-\mathrm{MS}$;

$\mathrm{b}=\operatorname{sqrt}\left(\mathrm{A}^{\prime}{ }^{*} \mathrm{C} \operatorname{inv} \mathrm{A}^{\star} \mathrm{A}\right)$

function $[\mathrm{LS}, \mathrm{C}]=$ limit_state $(\mathrm{L}, \mathrm{x})$

$\mathrm{C}=[]$;

$\mathrm{LS}=\mathrm{L}-\left(\left(\mathrm{x}(1)^{\wedge} 2-\mathrm{x}(2)^{\wedge} 2\right) /\left(2{ }^{*} \mathrm{x}(3){ }^{*} \mathrm{x}(4)\right)\right) ;$

ollipsoid approach on all limited SCLs

$\circ \mathrm{x}(1)=$ initial or diverge speed at the SCL

$\% x(2)=$ final or end speed at the SCL

$\circ \mathrm{x}(3)=$ Deceleration rate

$\circ \mathrm{M} \times(1)=$ mean initial or diverge speed at the SCL

$\circ \mathrm{M} x(2)=$ mean final or end speed at the SCL

${ }^{\circ} \mathrm{M}^{-} \mathrm{X}(3)=$ mean deceleration rate at the $\mathrm{SCL}$

$\circ \mathrm{M}^{-} \mathrm{X}(4)=$ mean Utilization rate at the $\mathrm{SCL}$

$\frac{\circ \bar{d}}{x}(1)=$ std of initial or diverge speed at the SCL

o Sd $\mathrm{x}(2)=$ std of final or end speed at the SCL

oSd $x(3)=$ std of deceleration rate at the SCL

o Sd_x $(4)=$ std of Utilization rate at the SCL

function [adjusted_data] = InputAll(filename, sheet, xlRange)

filename = '2013-09-16 Adjusted Data - Only positive Deceleration'; sheet $=7$;

xlRange $=$ 'R:U';

Lrange $=$ 'M2';

osheet: 1- Island Park, 2-Parkdale, 3-Bronson, 4-Moodie, 5-Terrfox ENS,

6- Carp, 7- Terryfox W-NS

Data $=$ xlsread (filename, sheet, xlRange) data input

Limit $=$ xlsread (filename, sheet, Lrange) $\%$ SCL length

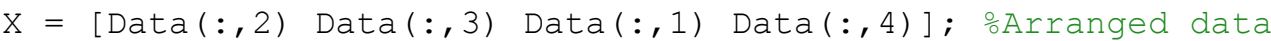




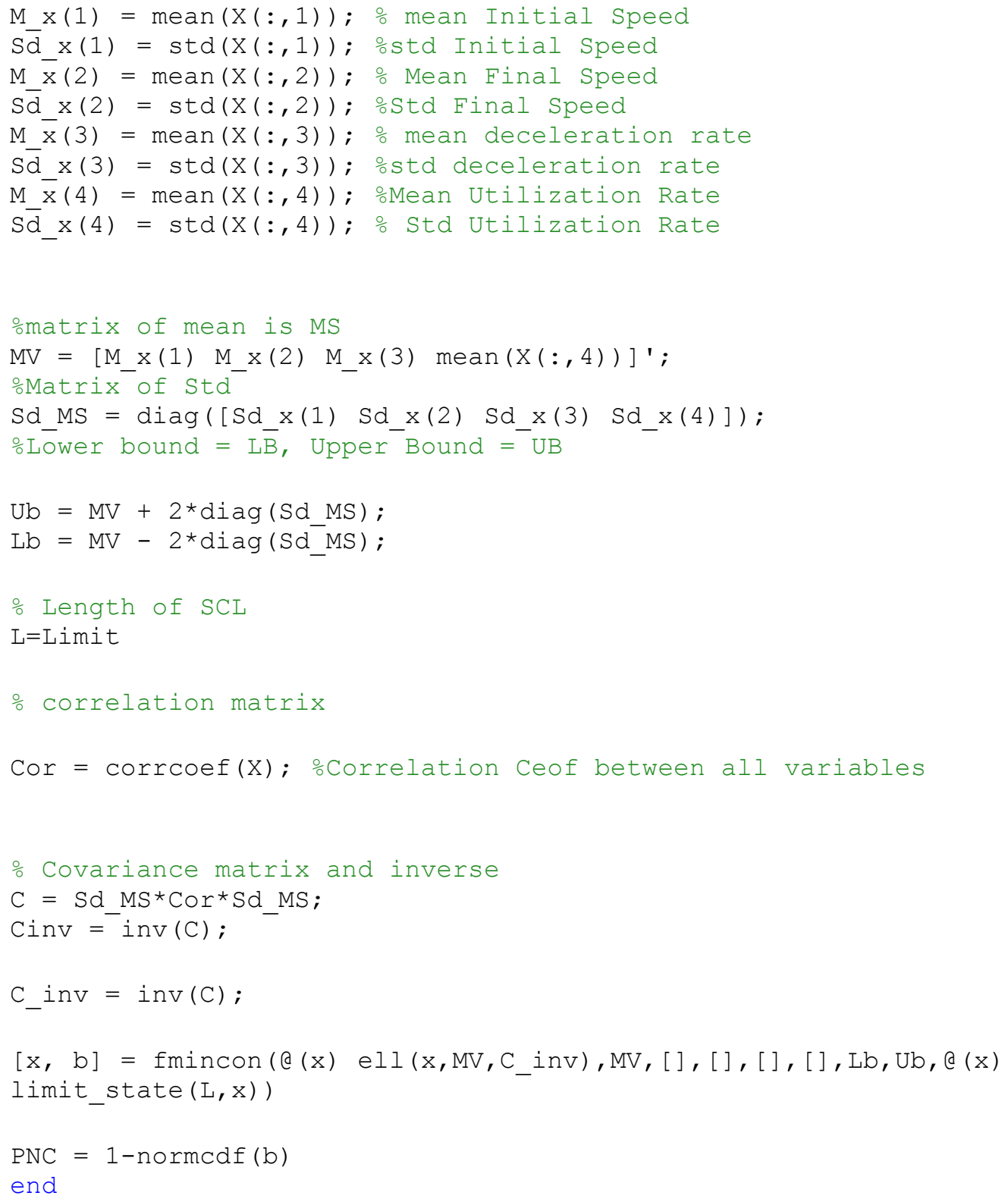




\section{Using All Sites Combined Data}

function $\mathrm{b}=\operatorname{ell}\left(\mathrm{x}, \mathrm{MS}, \mathrm{C}_{-}\right.$inv)

$\mathrm{A}=\mathrm{x}-\mathrm{MS}$;

$\mathrm{b}=\operatorname{sqrt}\left(\mathrm{A}^{\prime}{ }^{*} \mathrm{C}_{-} i n \mathrm{~V}^{\star} \mathrm{A}\right)$;

function $[\mathrm{LS}, \mathrm{C}]=$ limit state $(\mathrm{L}, \mathrm{X})$

$\mathrm{C}=[]$;

$\mathrm{LS}=\mathrm{L}-\left(\left(\mathrm{x}(1)^{\wedge} 2-\mathrm{x}(2)^{\wedge} 2\right) /\left(2{ }^{\star} \mathrm{x}(3)^{\star} \mathrm{x}(4)\right)\right) ;$

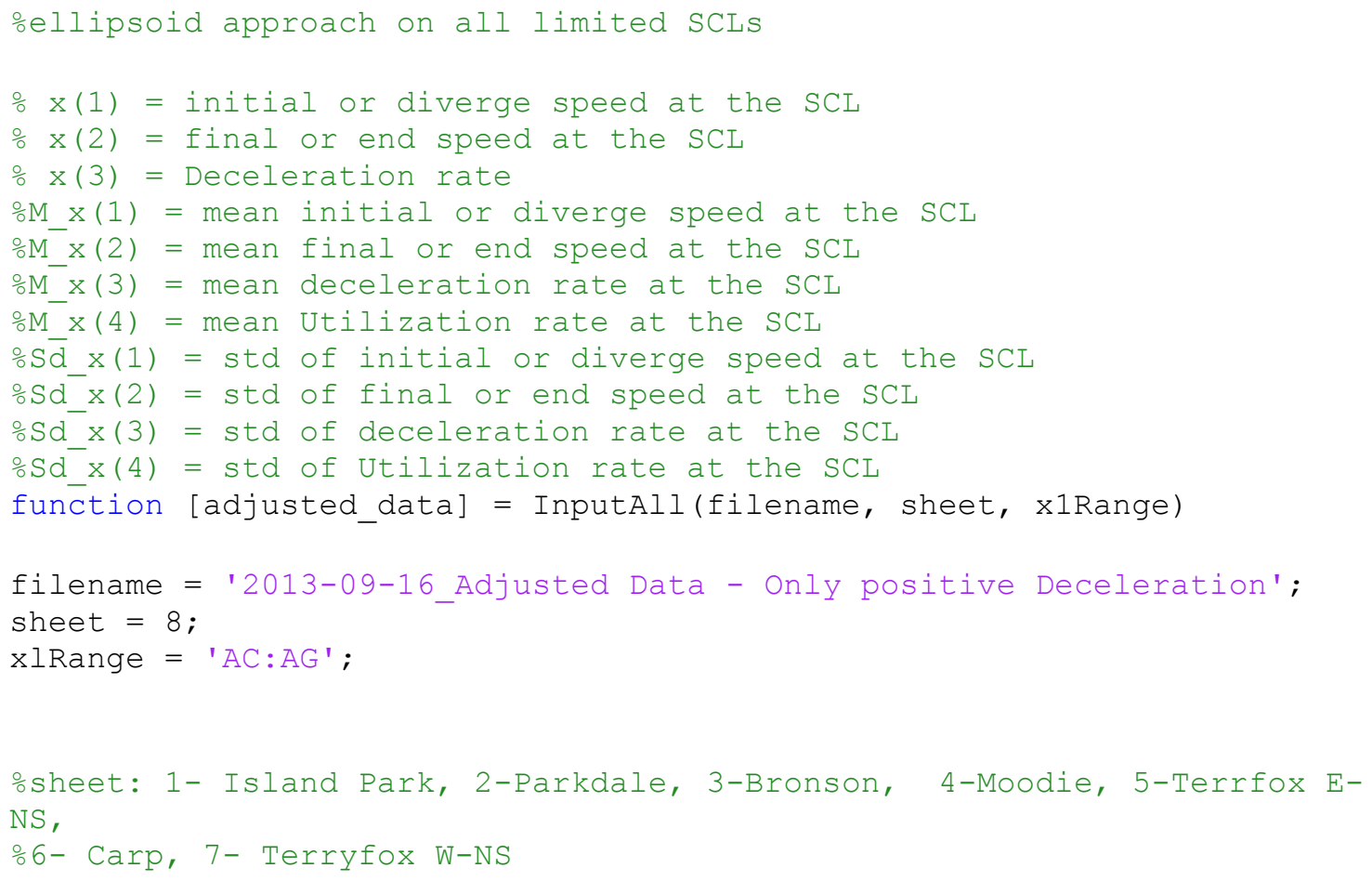




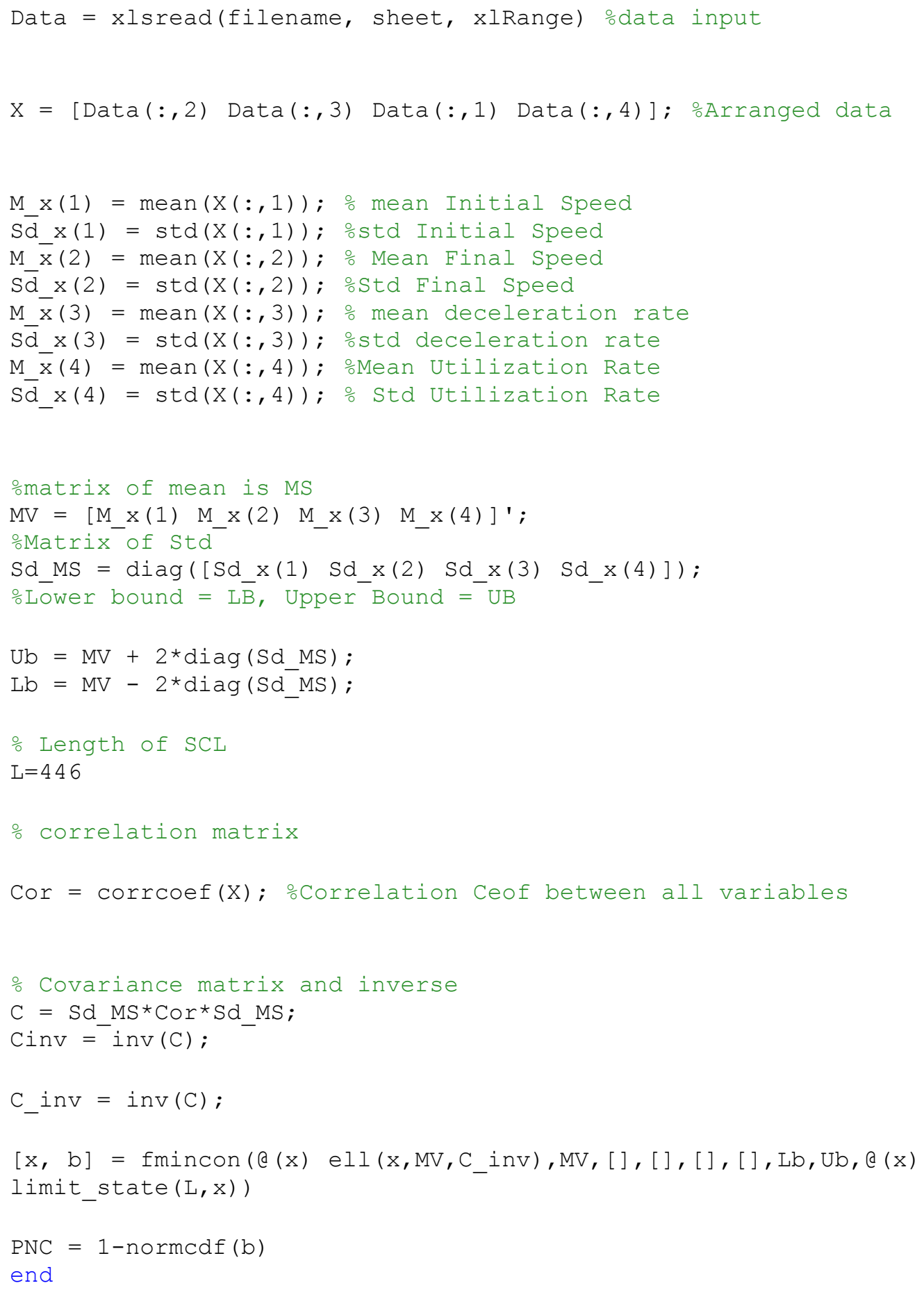


Appendix 11: MCS Design Application Script. 


\section{Using All Sites Combined Data}

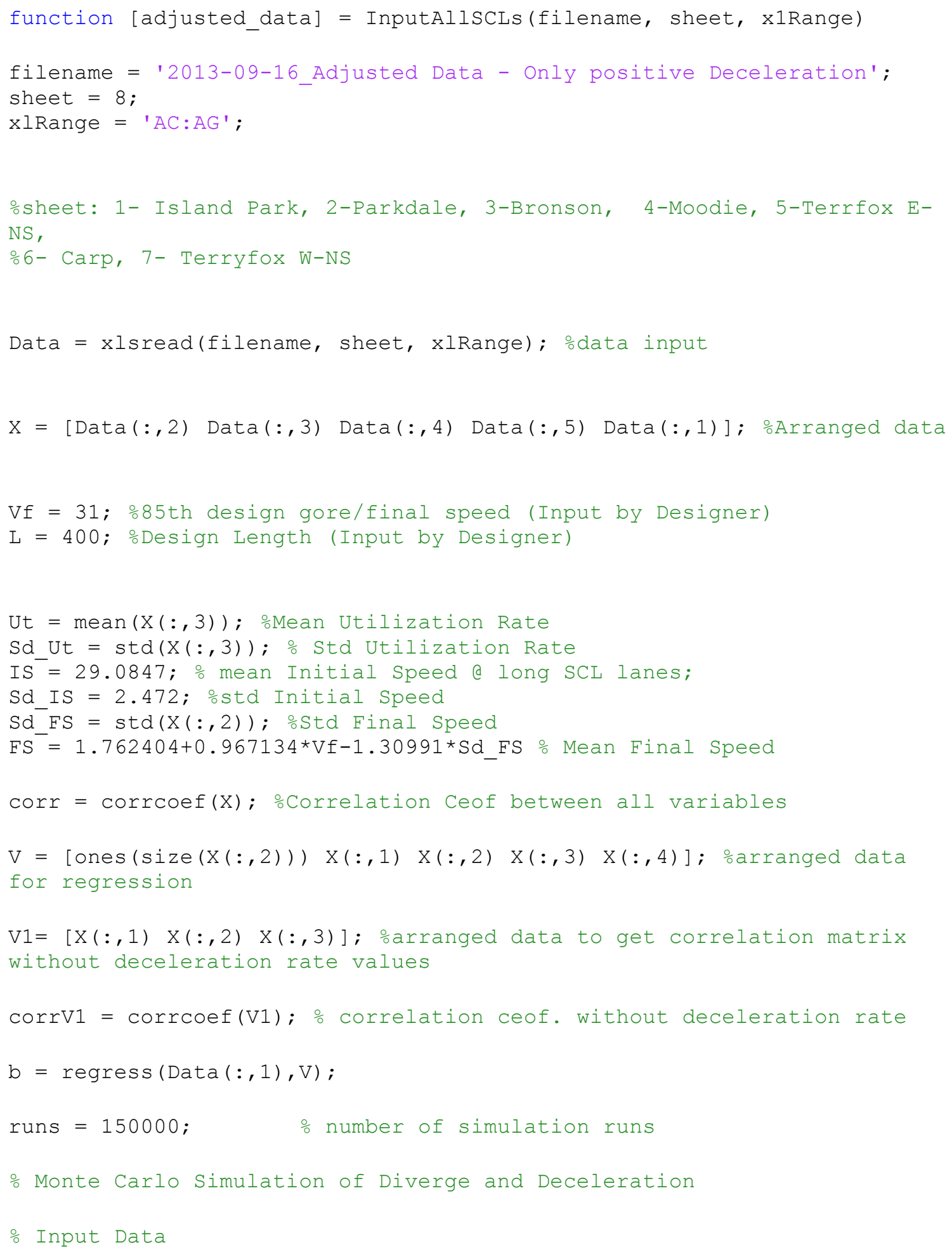




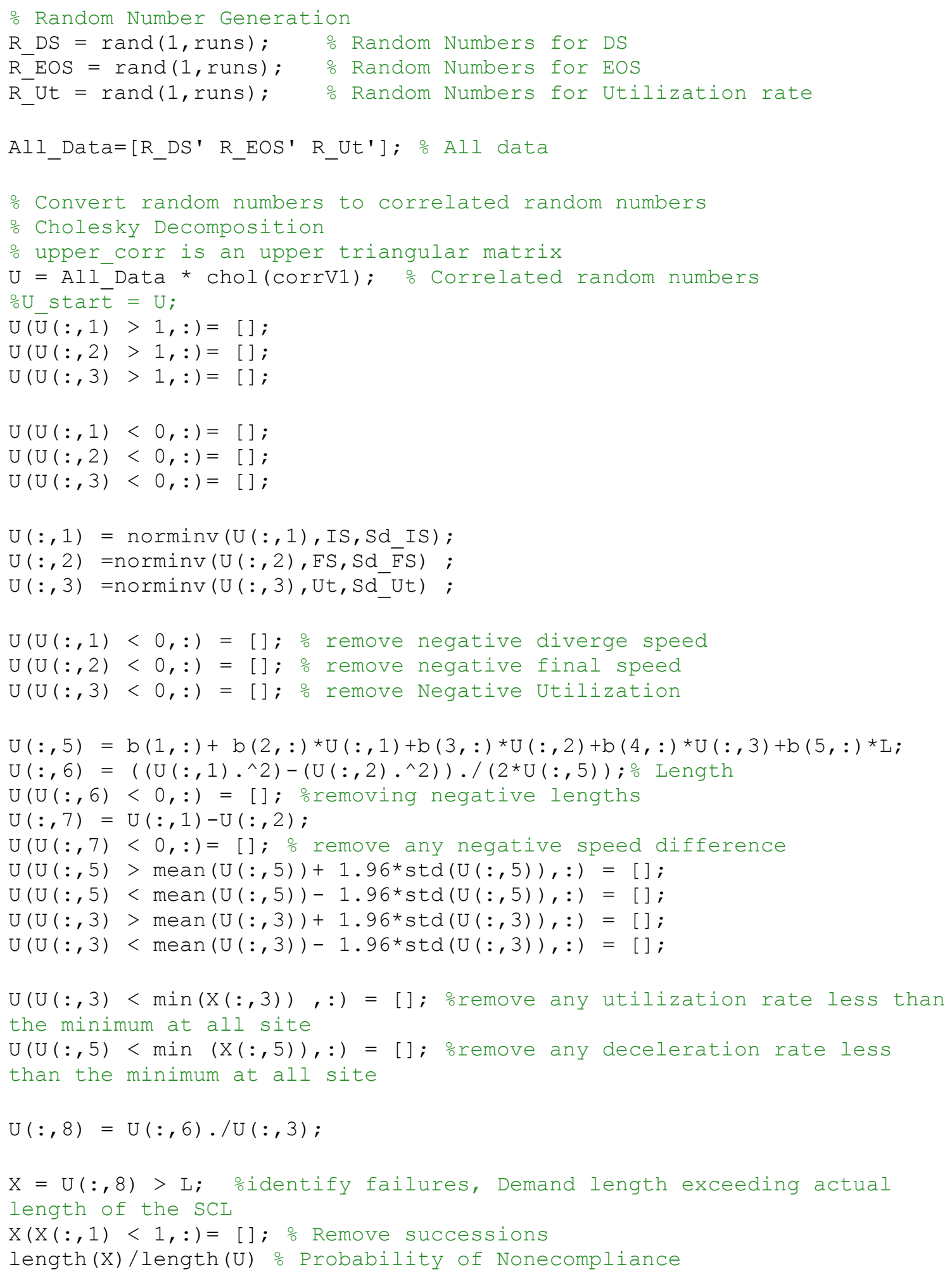

end 
Appendix 12: Speed Profiles 


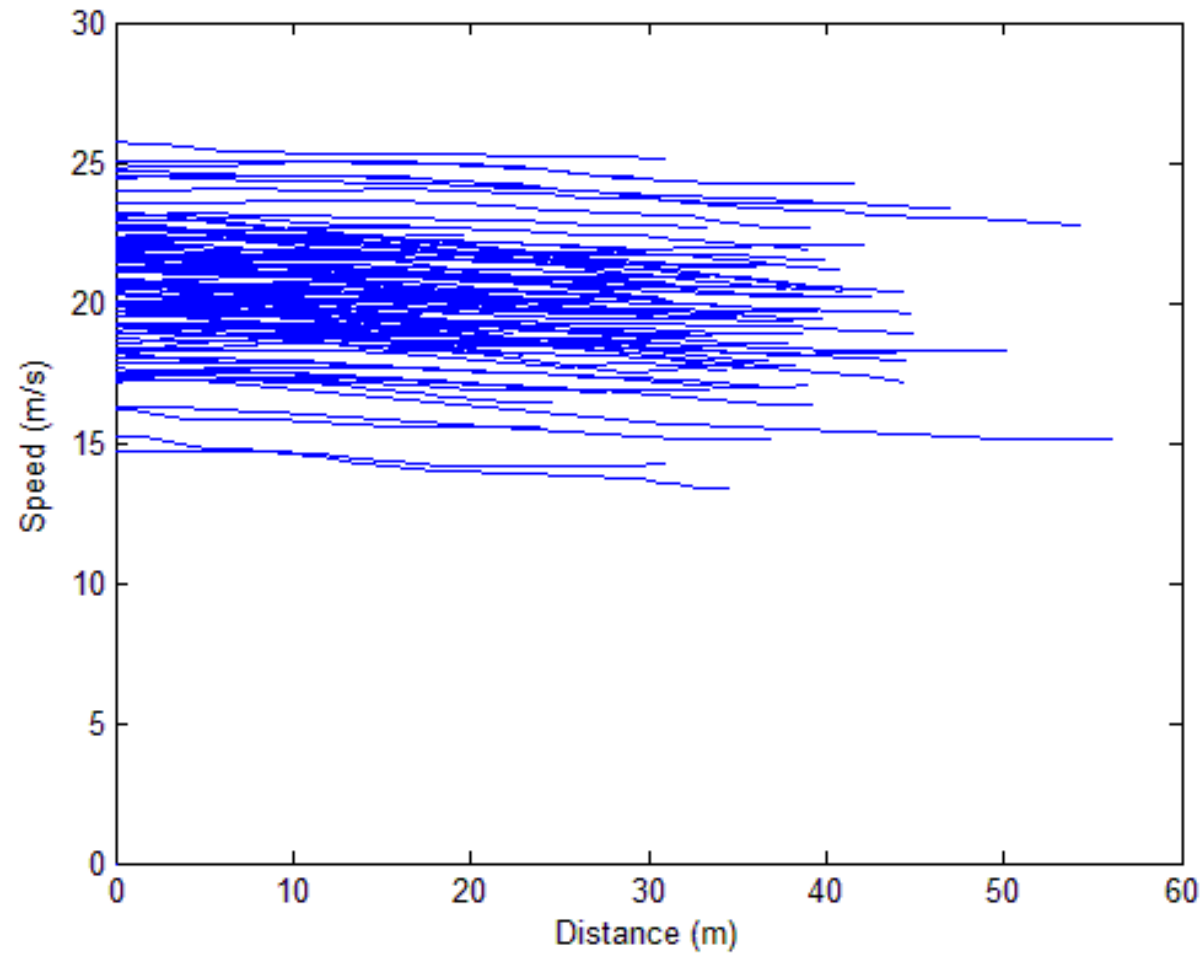

Figure 25: Speed Profiles of Vehicles at Island Park Drive E-N

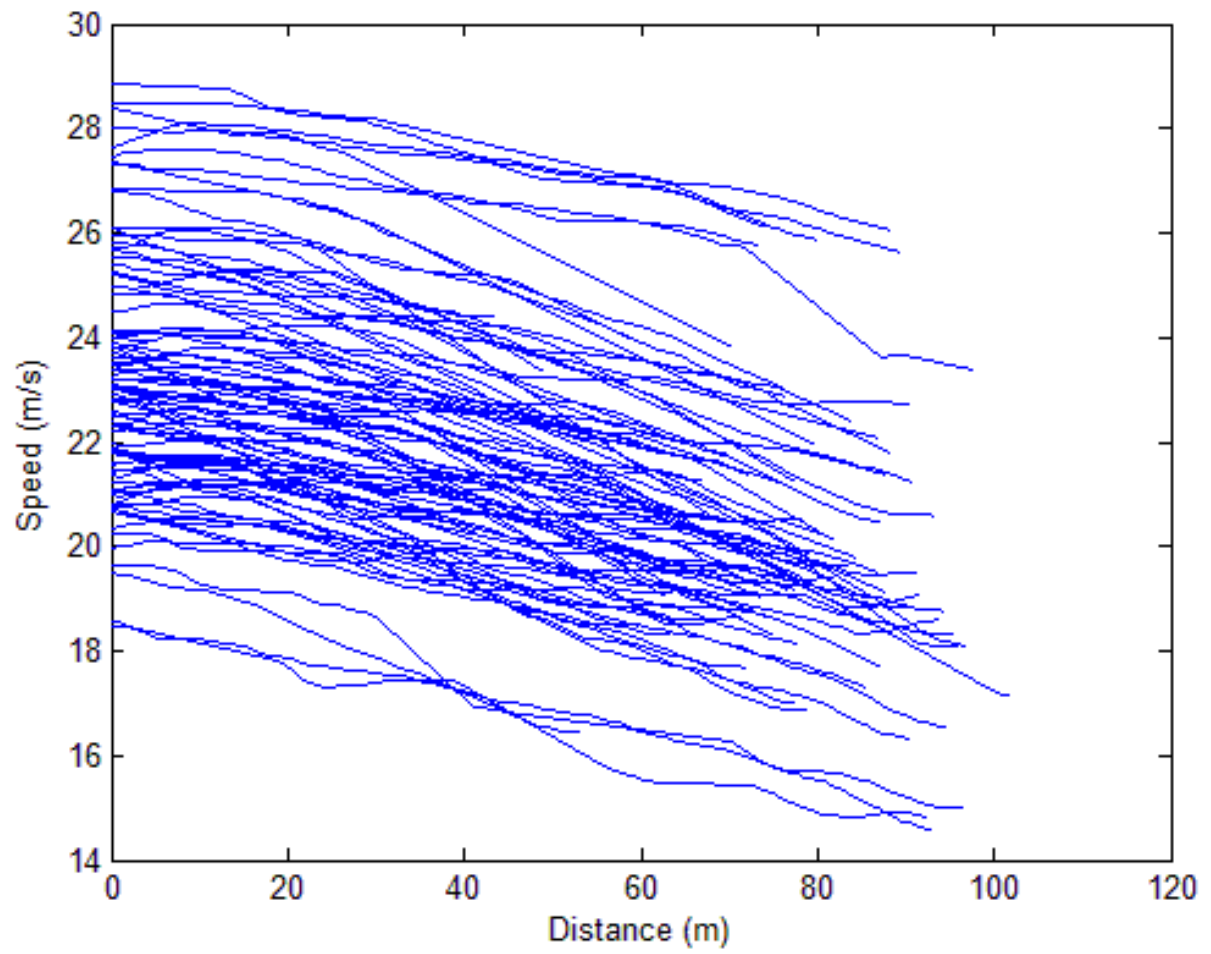

Figure 26: Speed Profiles of Vehicles at Parkdale Avenue W-NS. 


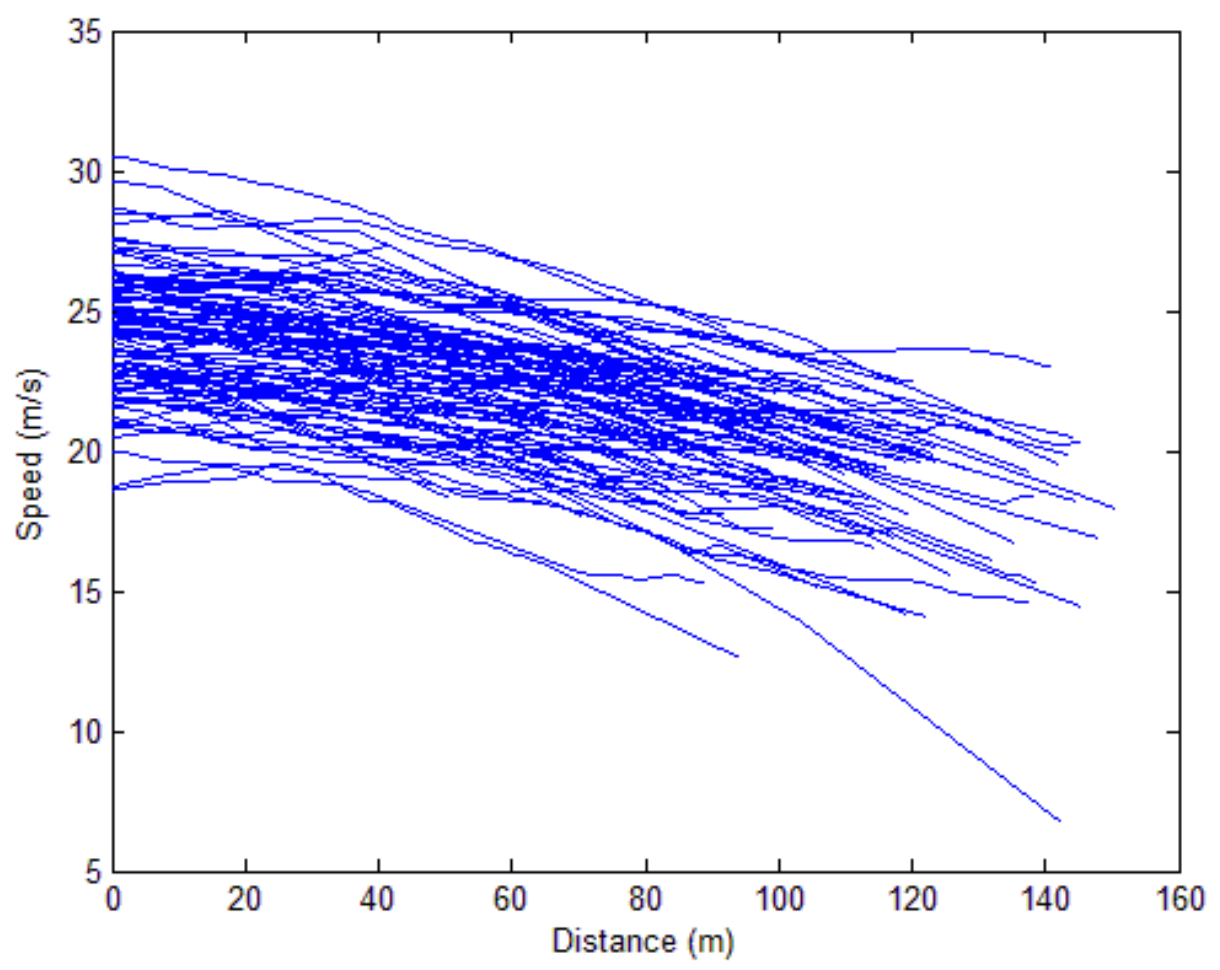

Figure 27: Speed Profiles of Vehicles at Bronson Avenue W-NS.

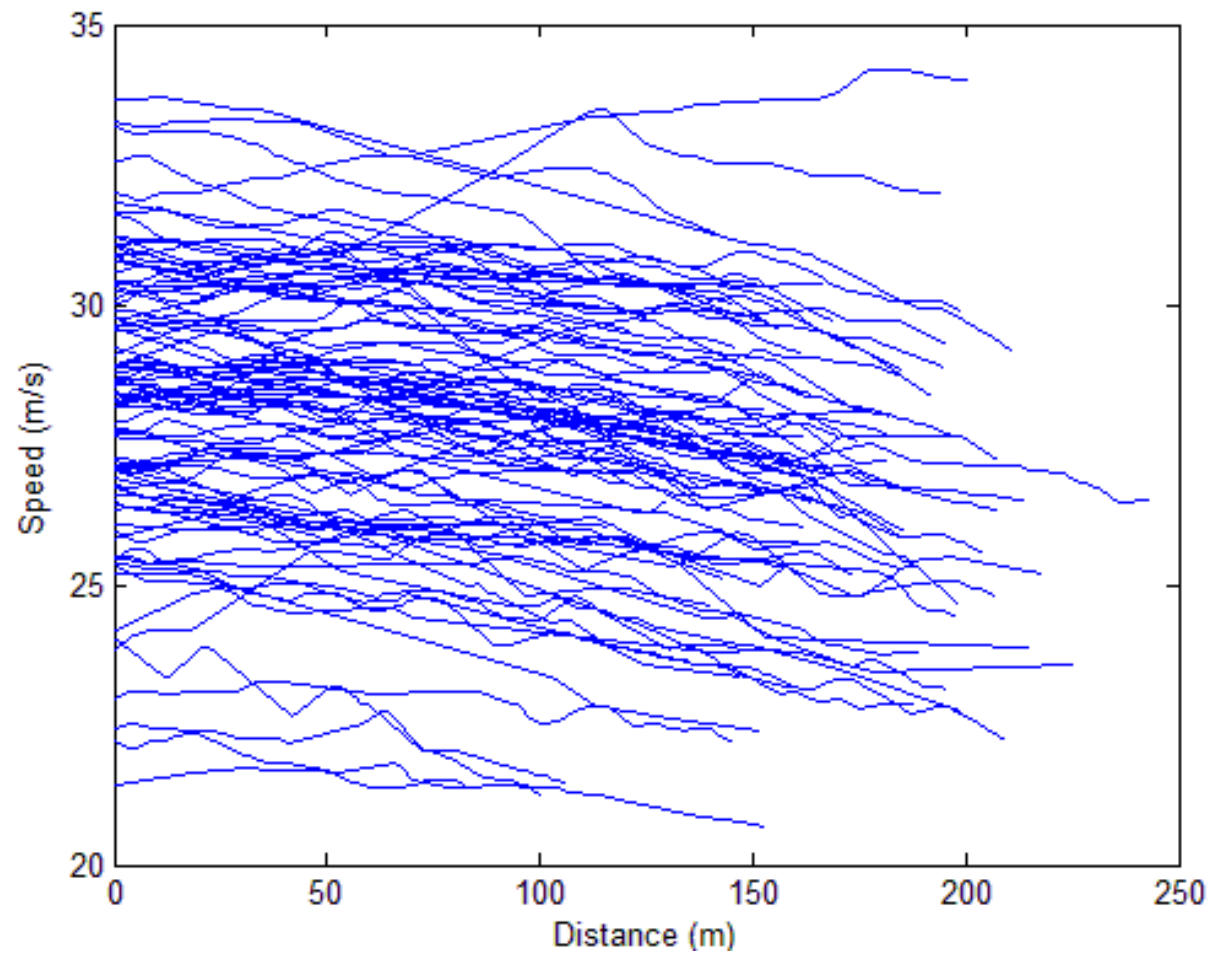

Figure 28: Speed Profiles of Vehicles at Moodie Drive W-NS. 


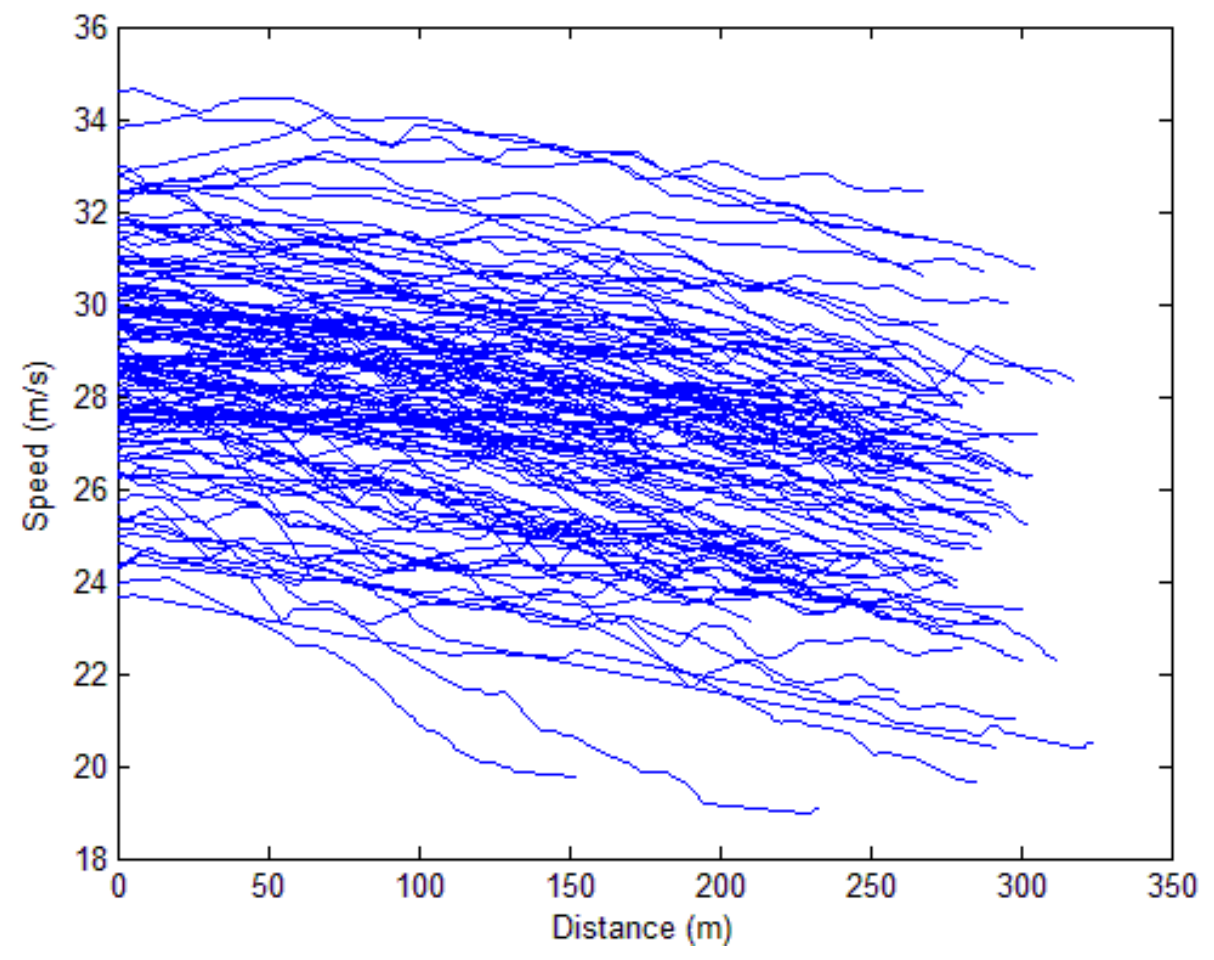

Figure 29: Speed Profiles of Vehicles at Terry Fox Drive E-NS.

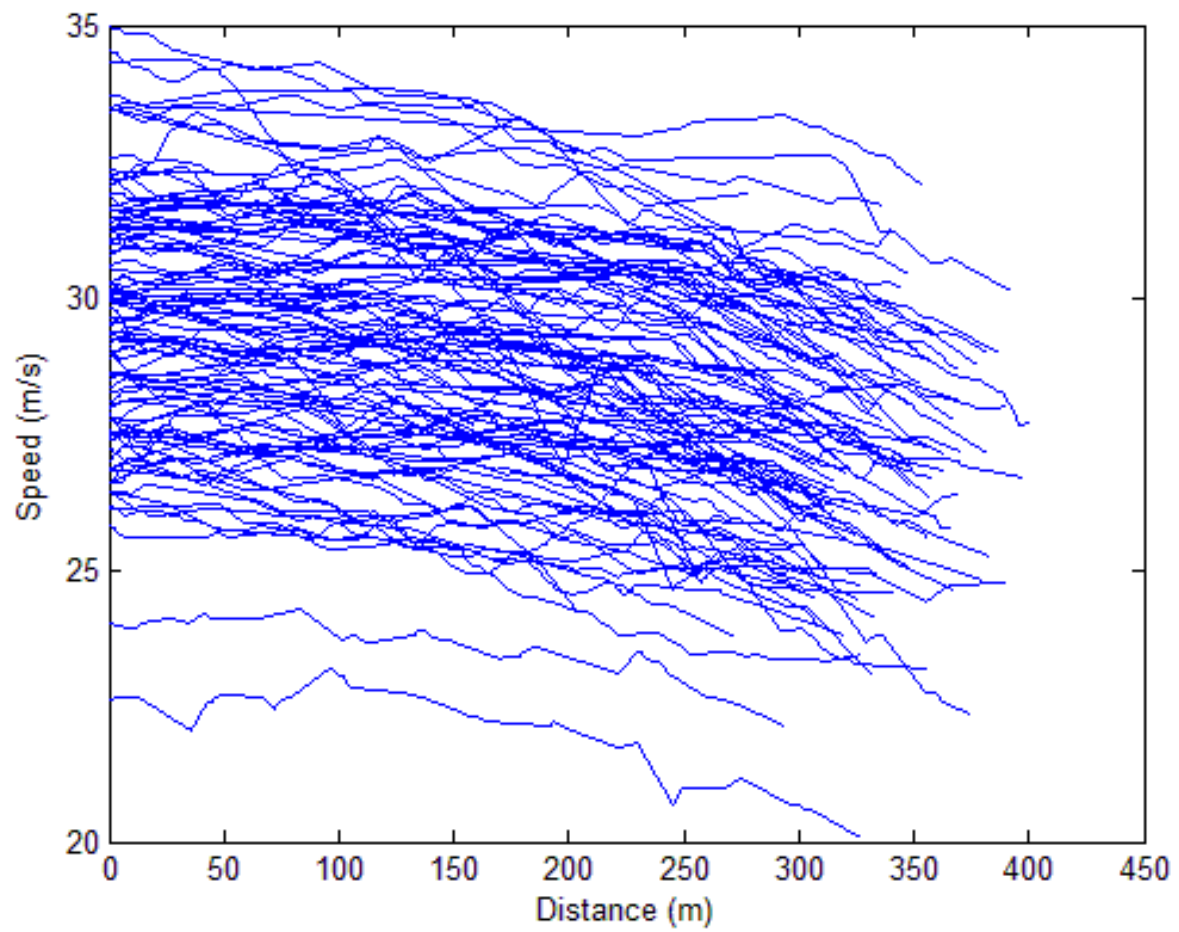

Figure 30: Speed Profiles of Vehicles at Carp Road E-NS. 


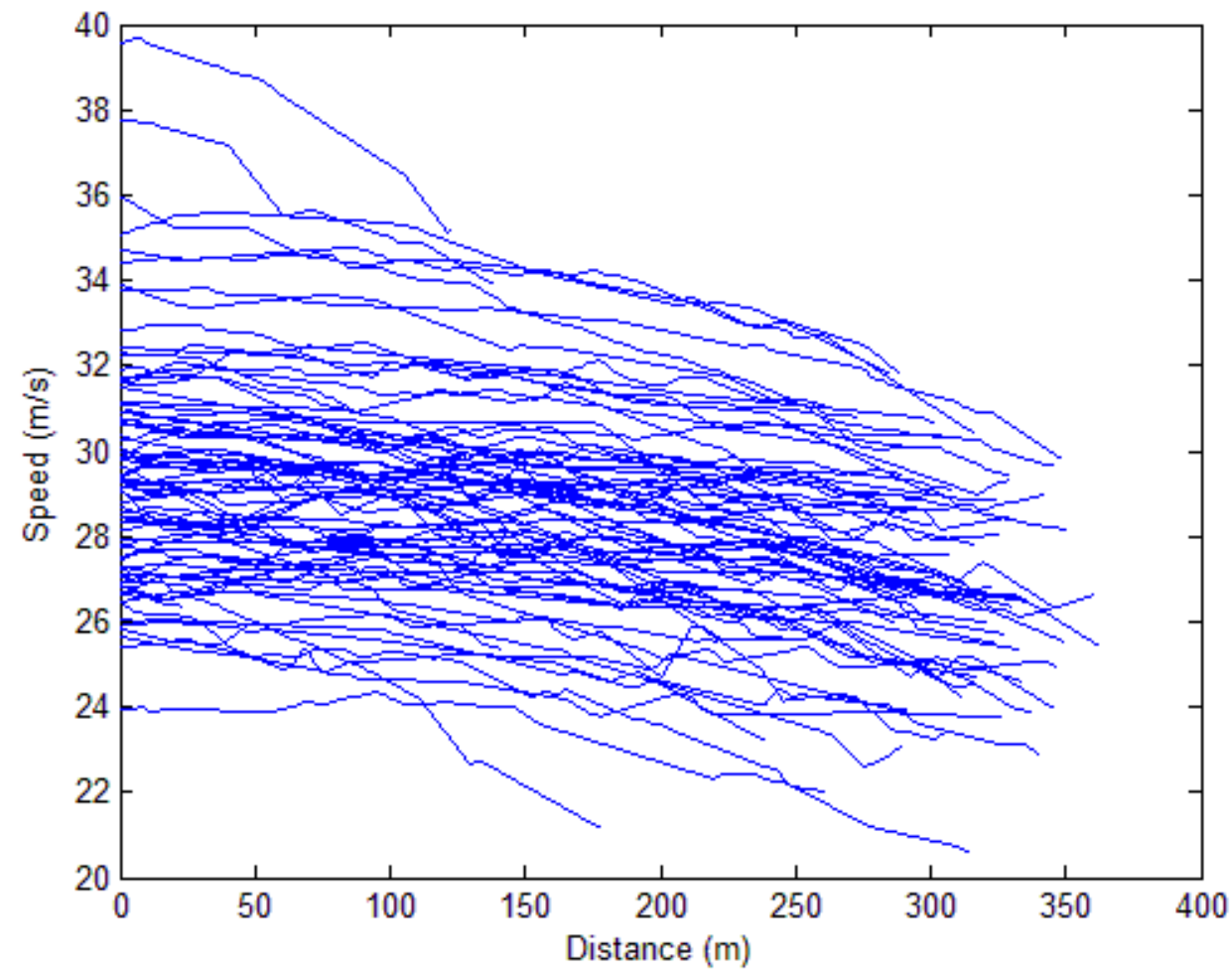

Figure 31: Speed Profiles of Vehicles at Terry Fox Drive W-NS. 


\section{Appendix 13: Frequency of Demand Length Output for MCS using the Dataset of Each Site.}




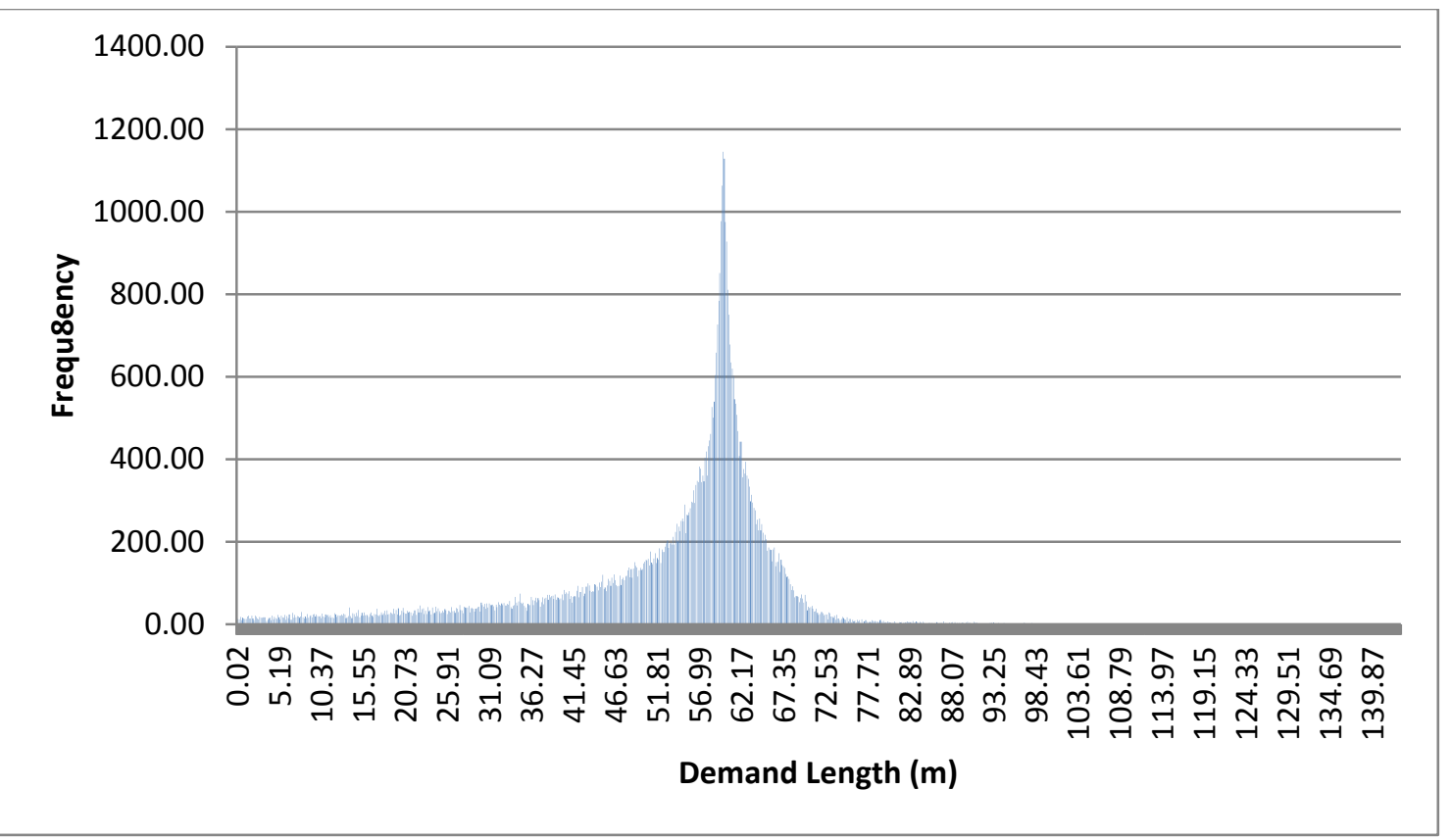

Figure 32: Demand Length Histogram for Island Park Drive E-N.

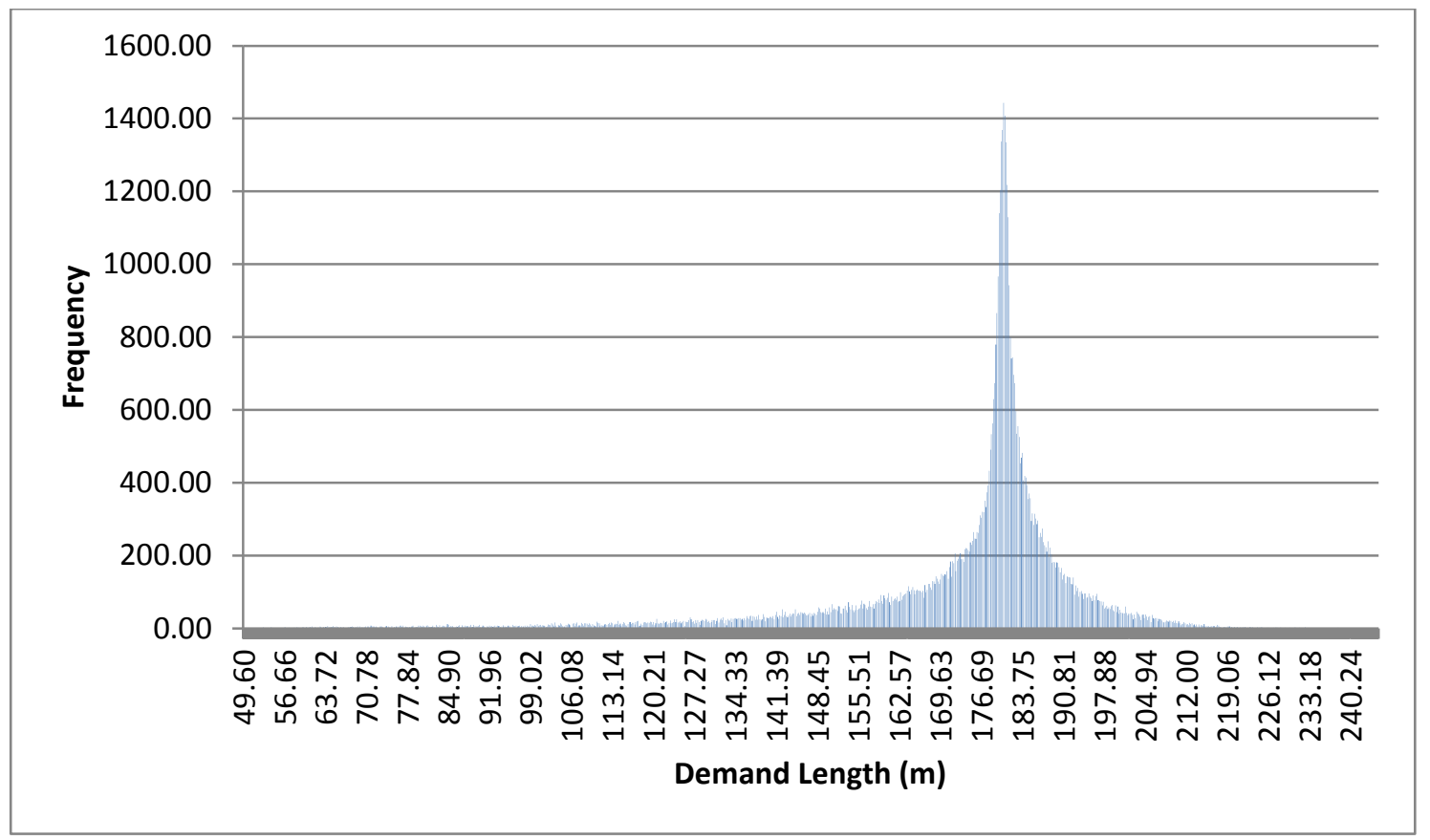

Figure 33: Demand Length Histogram for Parkdale Avenue W-NS. 


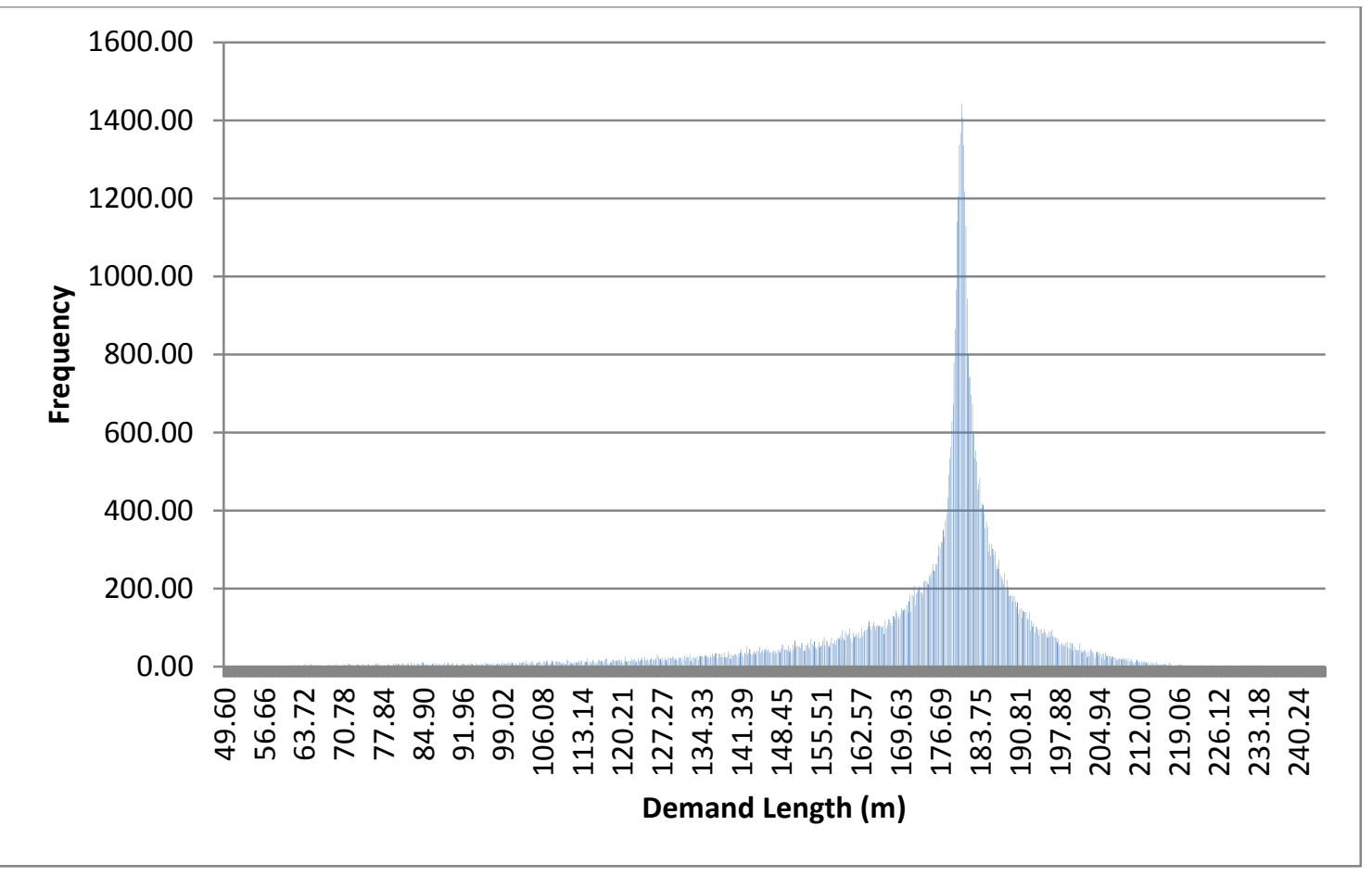

Figure 34: Demand Length Histogram for Bronson Avenue W-NS.

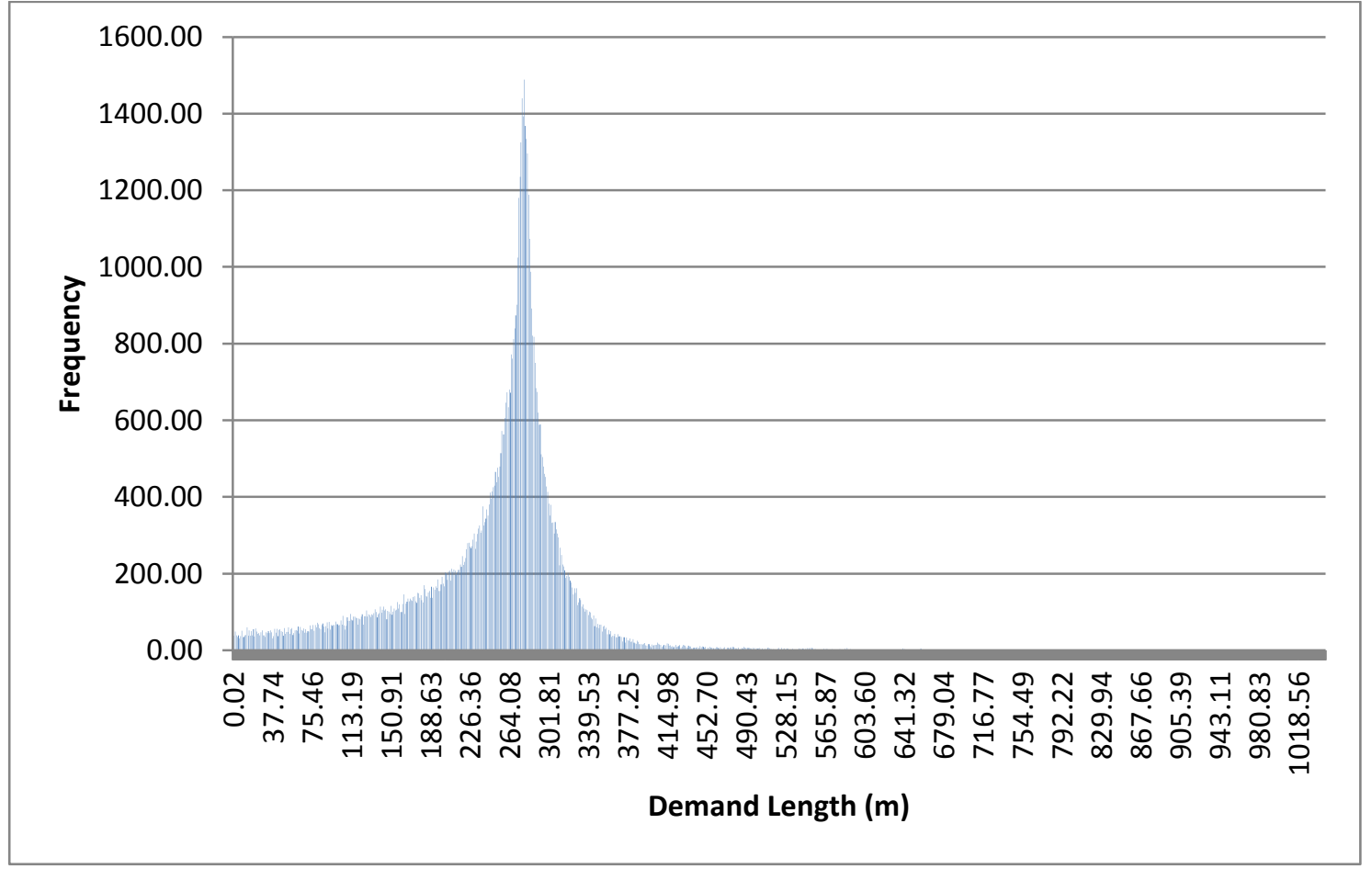

Figure 35: Demand Length Histogram for Moodie Drive W-NS. 


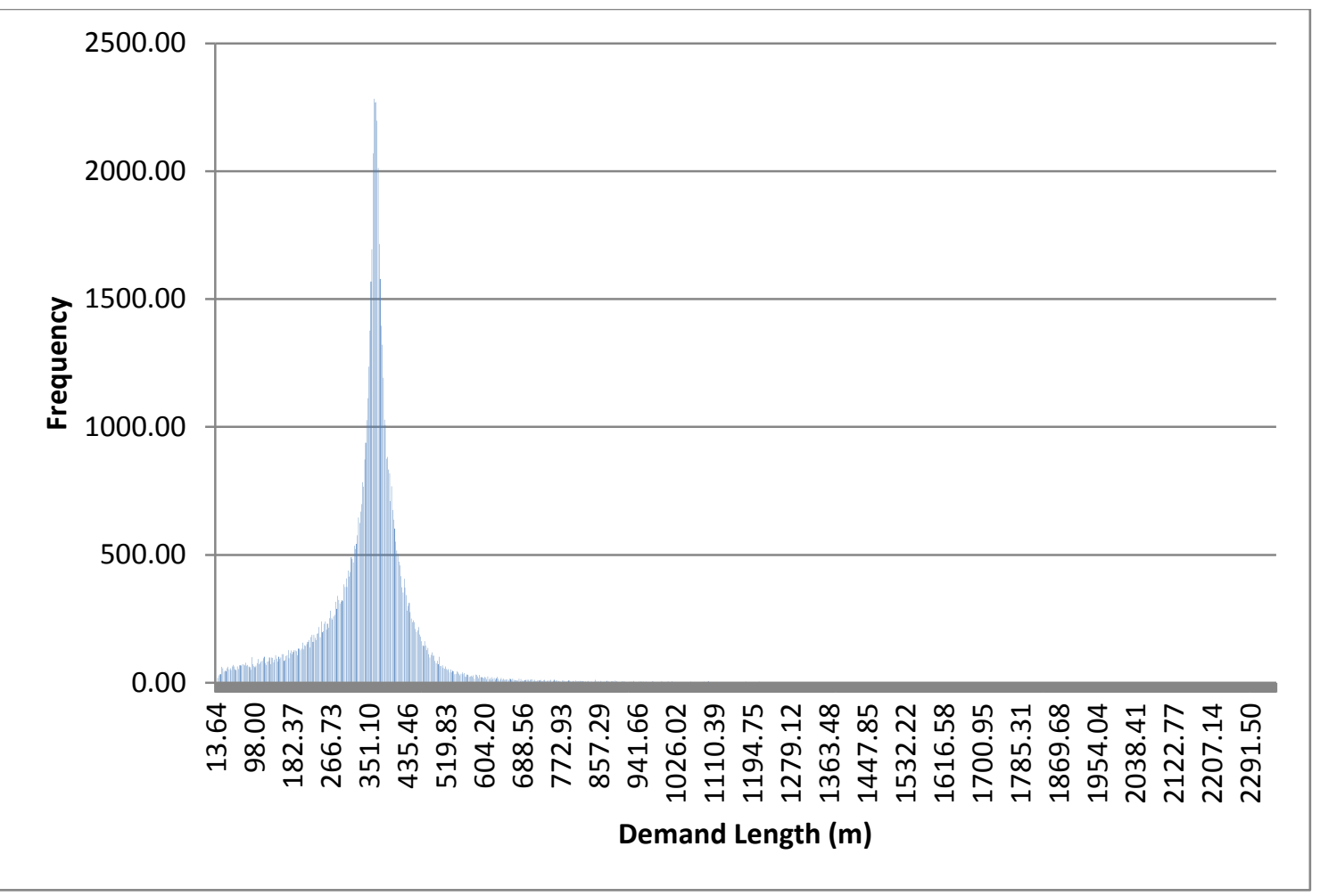

Figure 36: Demand Length Histogram for Terry Fox Drive E-NS.

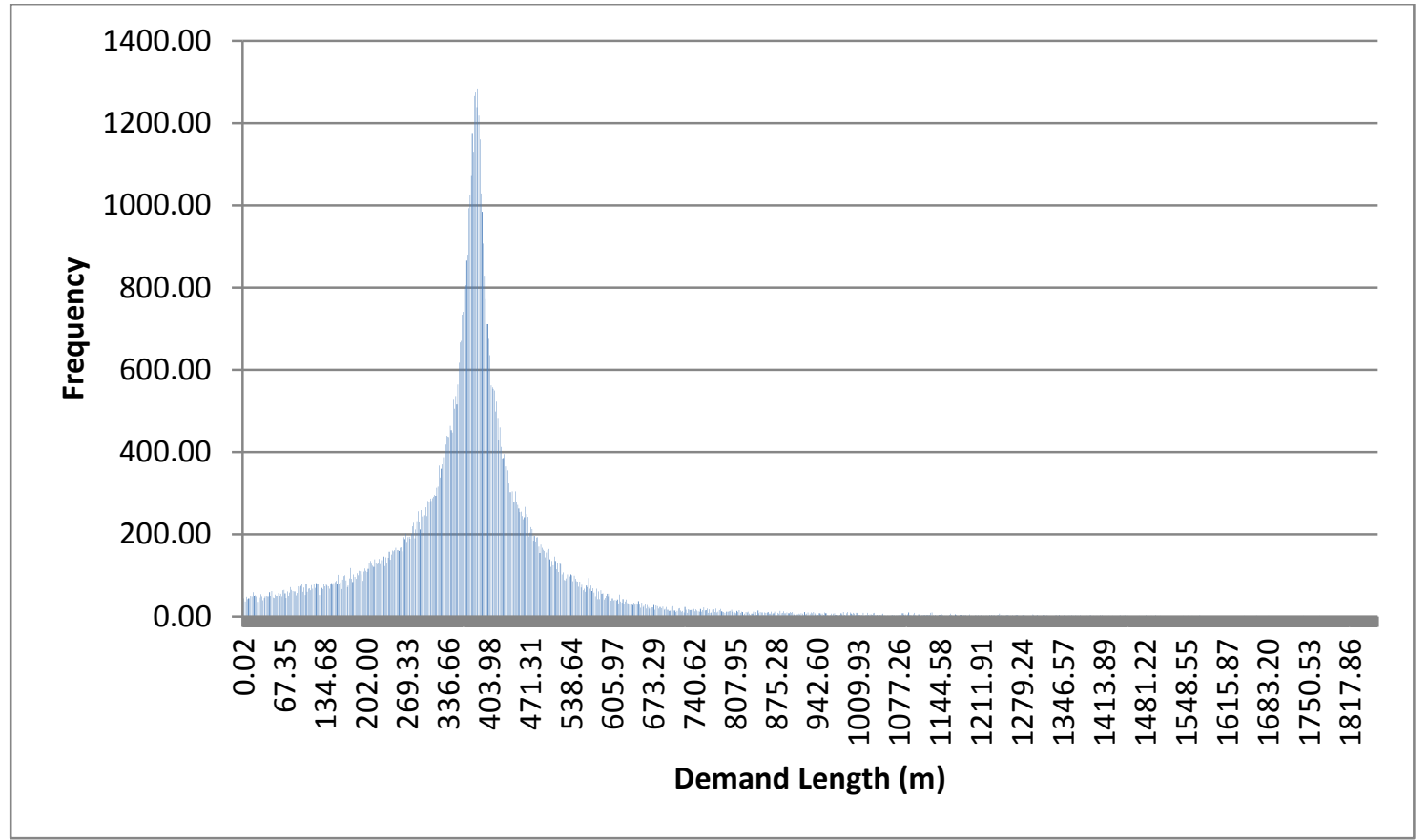

Figure 37: Demand Length Histogram for Carp Road E-NS. 


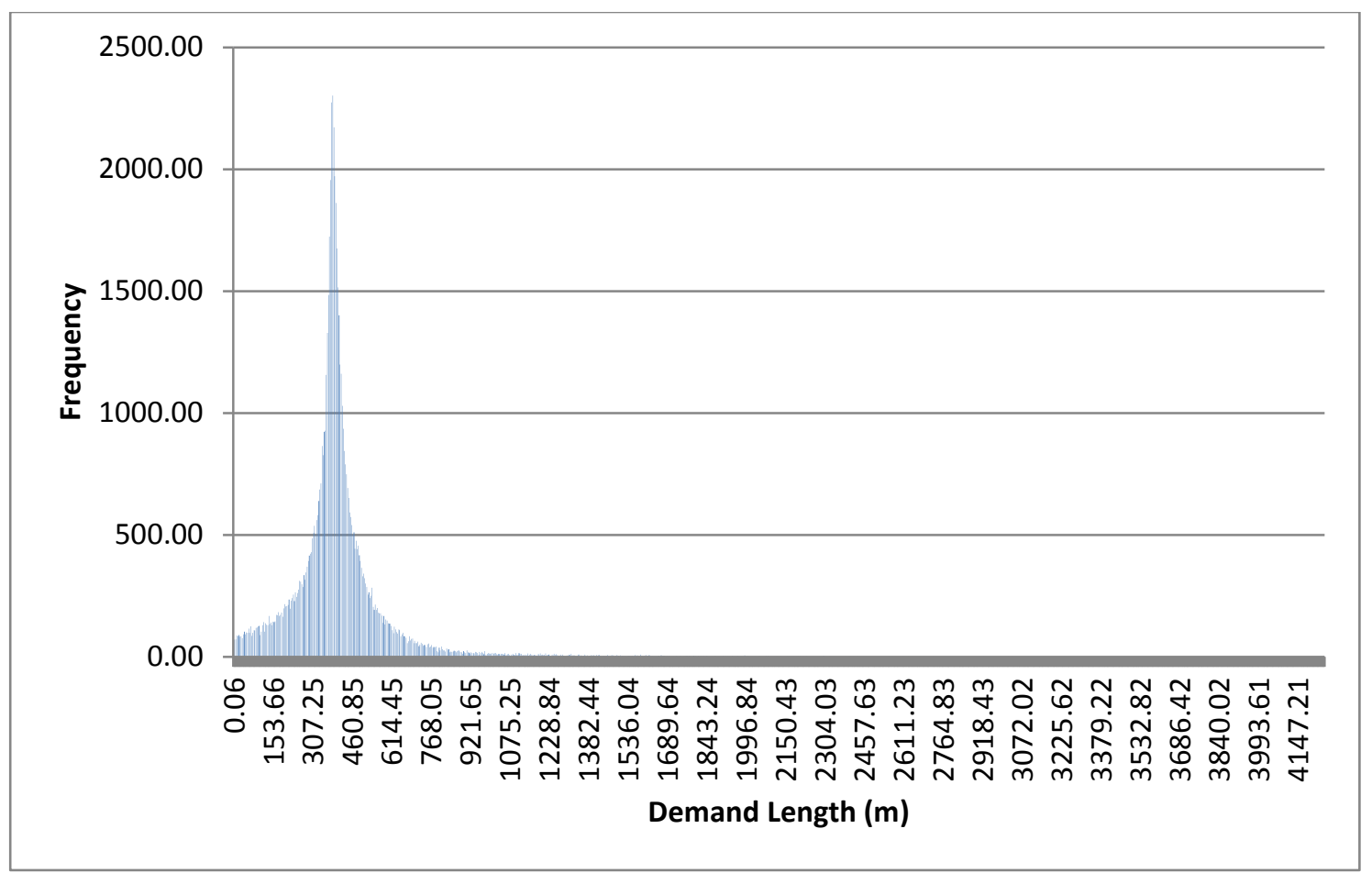

Figure 38: Demand Length Histogram for Terry Fox Drive W-NS. 